University of Louisville

ThinkIR: The University of Louisville's Institutional Repository

Electronic Theses and Dissertations

$5-2008$

\title{
Mining and tracking evolving web user trends from very large web server logs.
}

Basheer Hawwash 1984-

University of Louisville

Follow this and additional works at: https://ir.library.louisville.edu/etd

\section{Recommended Citation}

Hawwash, Basheer 1984-, "Mining and tracking evolving web user trends from very large web server logs." (2008). Electronic Theses and Dissertations. Paper 588.

https://doi.org/10.18297/etd/588

This Master's Thesis is brought to you for free and open access by ThinkIR: The University of Louisville's Institutional Repository. It has been accepted for inclusion in Electronic Theses and Dissertations by an authorized administrator of ThinkIR: The University of Louisville's Institutional Repository. This title appears here courtesy of the author, who has retained all other copyrights. For more information, please contact thinkir@louisville.edu. 


\title{
MINING AND TRACKING EVOLVING WEB USER TRENDS FROM VERY LARGE WEB SERVER LOGS
}

\author{
By \\ Basheer Hawwash \\ A Thesis \\ Submitted to the Faculty of the \\ Graduate School of the Lniversity of Louisville \\ in Partial Fulfillment of the Requirements \\ for the Degree of \\ Master of Science \\ Department of Computer Engineering and Computer Science \\ Lniversity of Louisville \\ Louisville. Kentucky
}

May 2008 


\title{
Mining and Tracking Evolving Web User Trends From Very Large Web Server Logs
}

\author{
By
}

Basheer Hawwash

A Thesis Approved on

April 10, 2008

by the following Thesis Committee:

Thesis Director: Dr. Olfa Nasraoui

Dr. Ibrahim Imam

Dr. Suraj Alexander 


\title{
ABSTRACT
}

\section{MINING AND TRACKING EVOLVING WEB USER TRENDS FROM VERY LARGE WEB SERVER LOGS}

\author{
$\mathrm{Br}$ \\ Basheer Hawwash
}

May 10. 2008

Online organizations are always in search for innovative marketing strategies to better satisfy their current website users and lure new ones. Thus, recently: many organizations have started to retain all transactions taking place on their website, and tried to utilize this information to better understand and satisfy their users. However. due to the huge amount of transaction data. traditional methods are neither possible nor cost-effective. Hence, the use of effective and automated methods to handle these transactions became imperative.

Web Lsage Mining is the process of applying data mining techniques on web log data (transactions) to extract the most interesting usage patterns. The usage patterns are stored as profiles (a set of URLs) that can be used in higher-lerel applications, e.g. a recommendation system. to meet the company's business goals. A lot of research has been conducted on Web Lsage Mining. howerer, little has been done to handle 


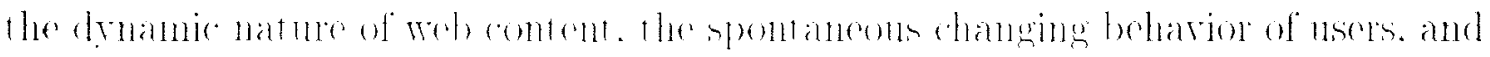

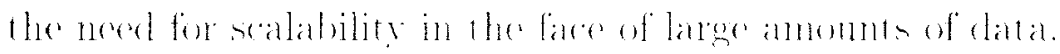

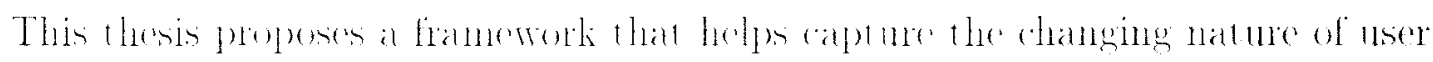

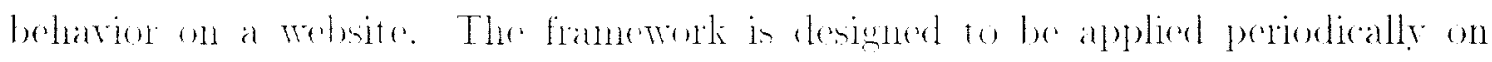

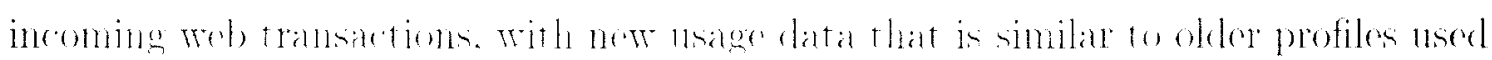

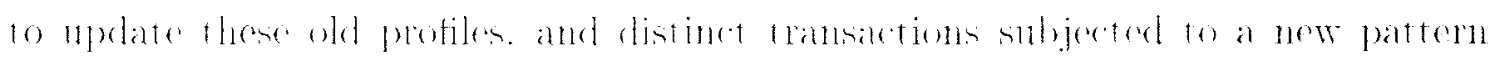

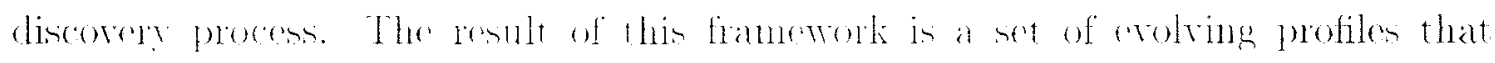

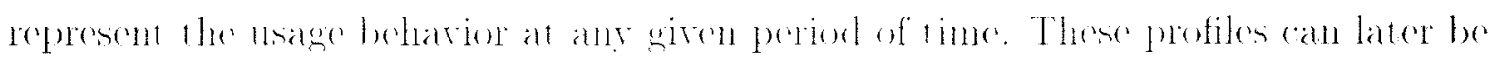
used in higher-level applications. for instance to purelied the wotring users interent as part of an intelligont werb promalization framework. 


\section{TABLE OF CONTENTS}

ABSTRACT $\quad$ iii

1 INTRODUCTION 1

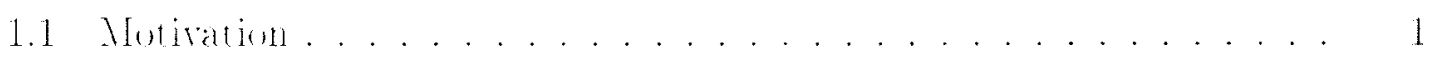

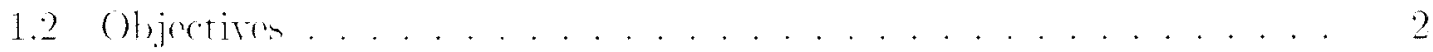

1.3 Thesis Contribution. . . . . . . . . . . . . . . . 4

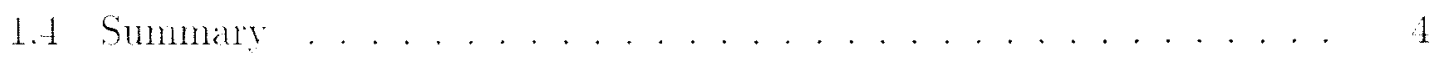

2 LITERATURE REVIEW $\quad 6$

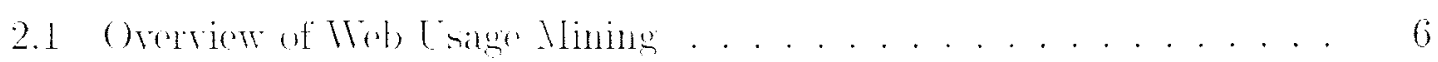

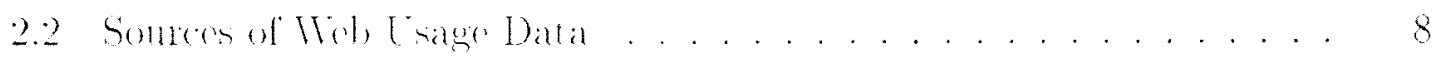

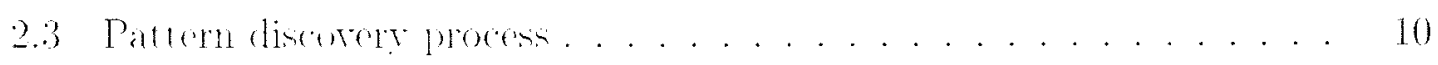

2.3.1 Preprocossing mage data . . . . . . . . . . . . 10

2.3 .2 Pattem Discort. . . . . . . . . . . . . . 13

2.3.3 Patrems Dnalysis . . . . . . . . . . . . . 16

2.1 HI $\mathrm{HC}^{\prime} \ldots \ldots \ldots \ldots \ldots \ldots \ldots \ldots$

2.t.1 Preporessing the mob log file to extract nore sessions . . . . 18

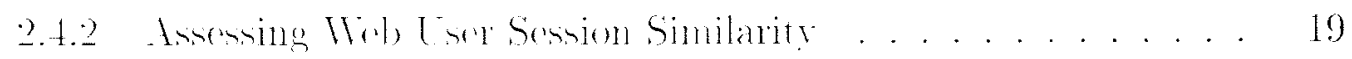

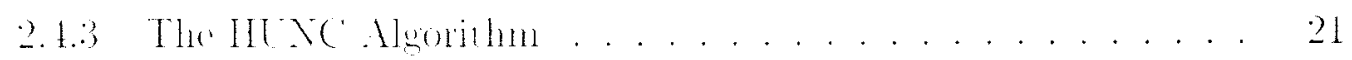

2.) Evolving Pruliles . . . . . . . . . . . . . . . . . . . 2.2

2.6 Scalabiliry . . . . . . . . . . . . . . . . . . . . . . . . .

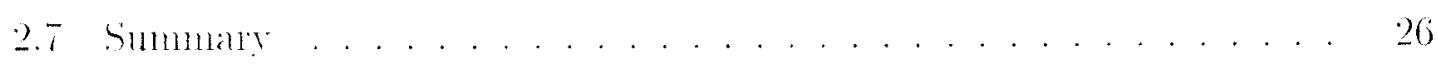




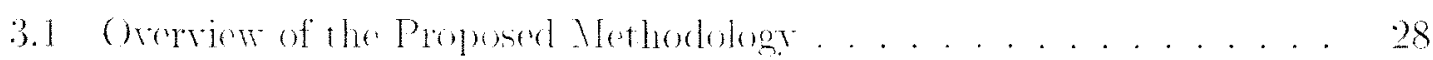

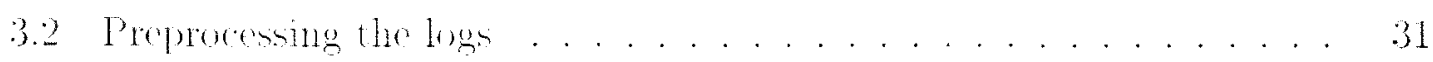

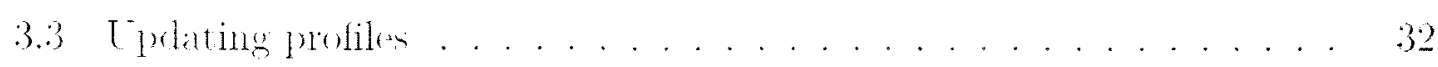

3.4 Discorering distinct profiles . . . . . . . . . . . . . 38

3.5 Post-proesening the distinct protiles . . . . . . . . . . . 39

3.6 Combining profitus . . . . . . . . . . . . . . . . . . 39

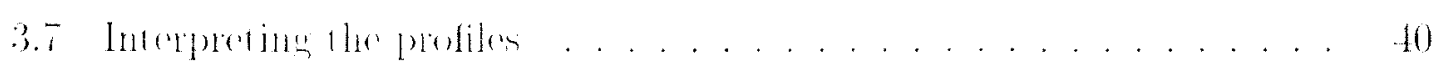

3.8 Profile Yaintriante. . . . . . . . . . . . . . . . . 11

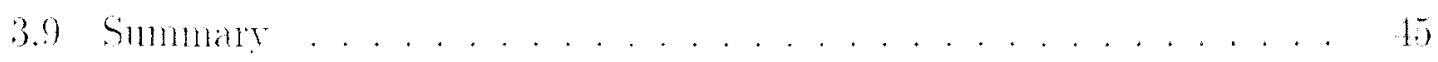

4 EXPERIMENTS 46

4.1 Exalnation $\backslash$ [otrics . . . . . . . . . . . . . . . . 46

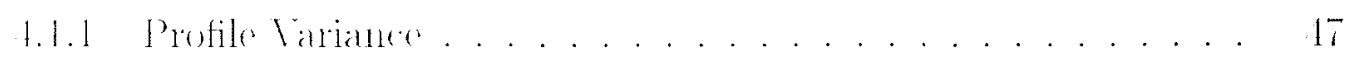

4.1 .2 Profik Cordinality ...................... 48

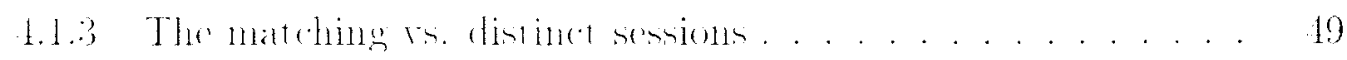

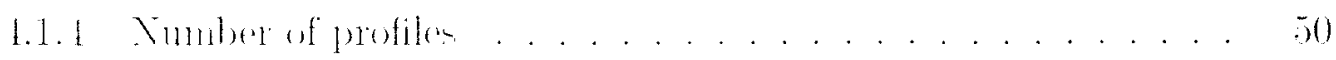

t.1.) Profile Pair-wior Similarity . . . . . . . . . . . . . .

4.1 .6 Protile Density . . . . . . . . . . . . . . . . . .

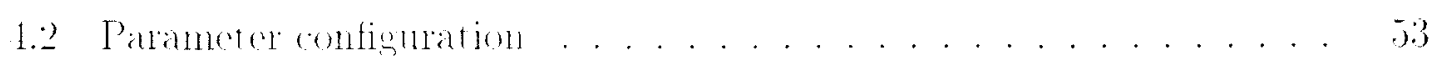

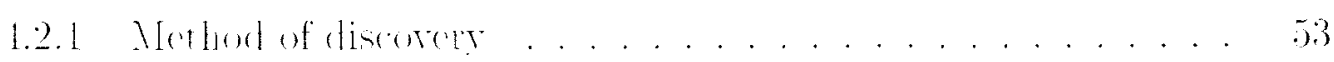

4.2.2 Ifothed of matching protiles . . . . . . . . . . . . .

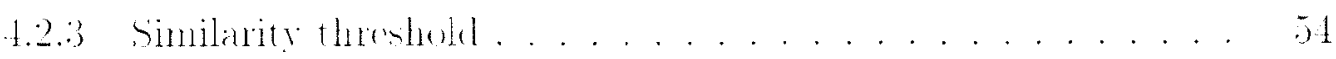

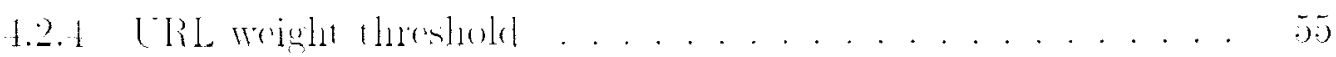

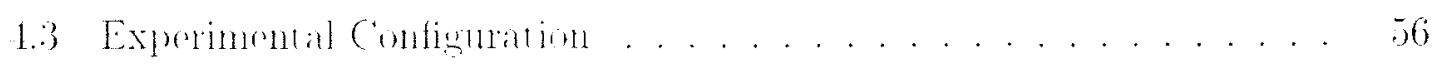

1.1 Experiment 1: Enirersity of Xissouri . . . . . . . . . 5

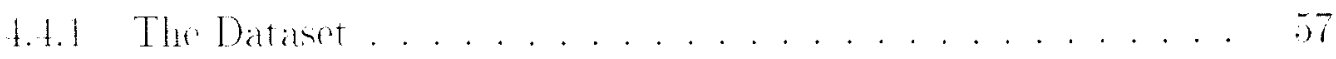

1.4.2 Protile Variances . . . . . . . . . . . . . 60 
4.4.3 Profile Erohtution ................. . . 64

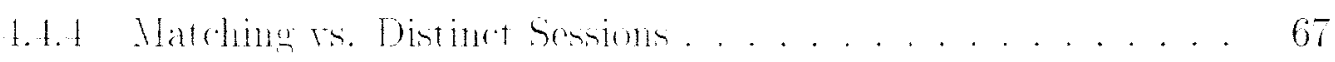

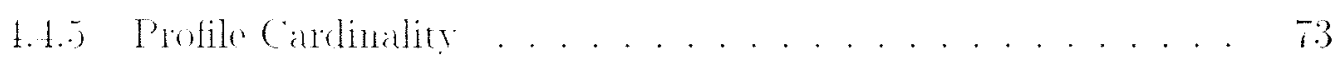

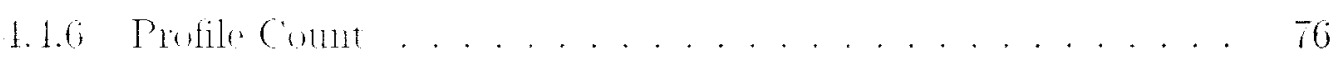

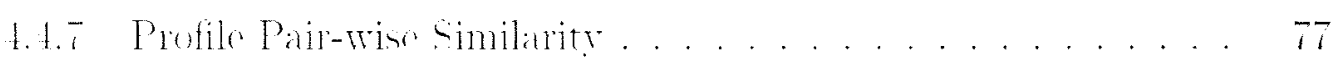

t.t.8 Profilo Densiry ......................... 78

4.4 Evolution rs. Thaditional (Full run) ........... . 81

4.5 Experinent 2: Coniremity of Lonimille Library . . . . . . . . . . 85

1.5.1 The Dataret . . . . . . . . . . . . . . . . . . . . .

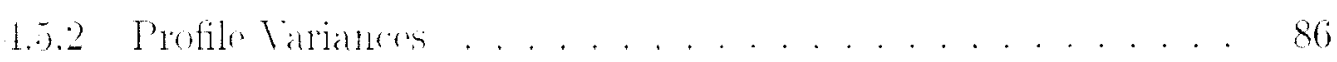

4.5 .3 profilo Erolution . . . . . . . . . . . . . 96

1.5.1 Matthing is. Distinct sessions . . . . . . . . . . . 97

1.5.5 Profile Cardinality . . . . . . . . . . . 101

4.5 .6 Profile Count . . . . . . . . . . . . . . 10.4

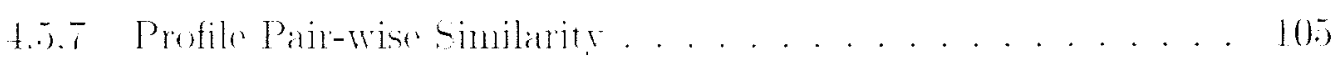

t.5.8 Profiln Density . . . . . . . . . . . . . 107

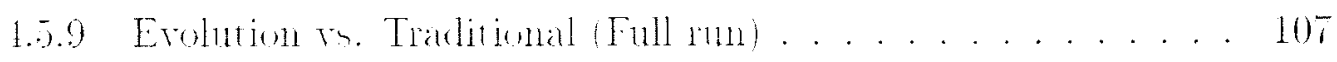

4.6 Experimental Design . . . . . . . . . . . . . . 113

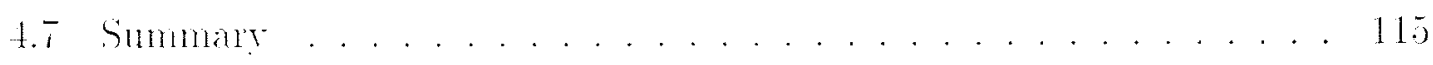

5 CONCLUSION 117

$\begin{array}{ll}\text { REFERENCES } & 120\end{array}$

$\begin{array}{ll}\text { APPENDIX A } & 125\end{array}$

$\begin{array}{ll}\text { CURRICULUM VITAE } & 144\end{array}$ 


\section{LIST OF TABLES}

$2.1 \log$ File Sanple . . . . . . . . . . . . . . . . . . . . 18

1.1 Evaluation Mertries Summary $(t-$ timo period index) . . . . . . t t t

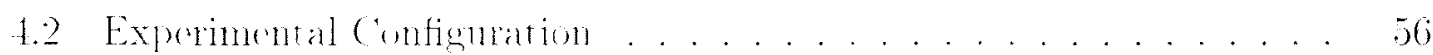

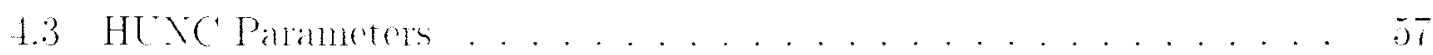

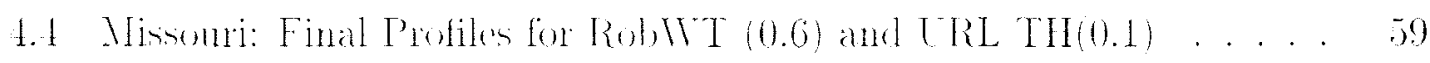

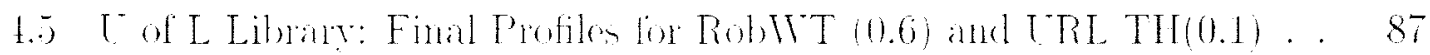

1.6 Experinental Dosign Farents . . . . . . . . . . . . . . 114

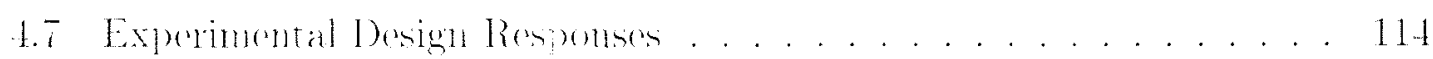

1.8 Experinemal Design Datal Table . . . . . . . . . . . 114 


\section{LIST OF FIGURES}

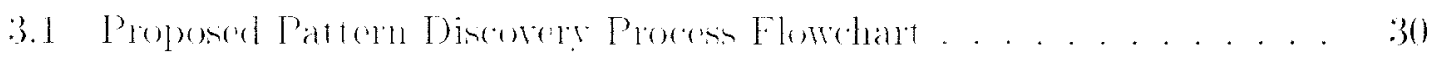

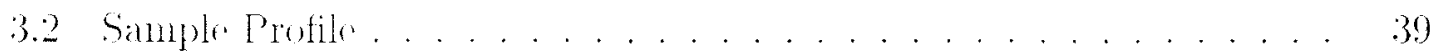

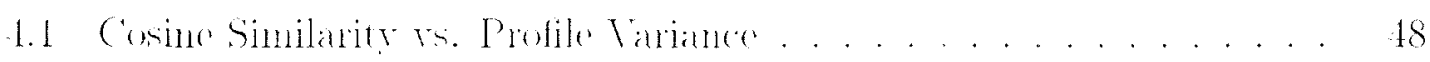

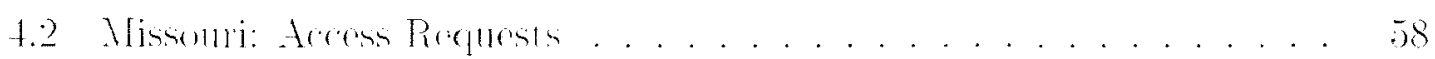

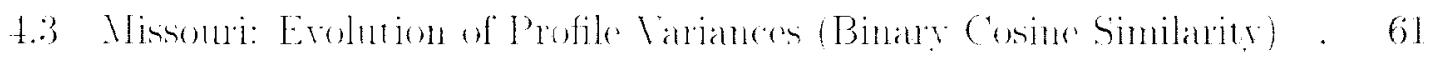

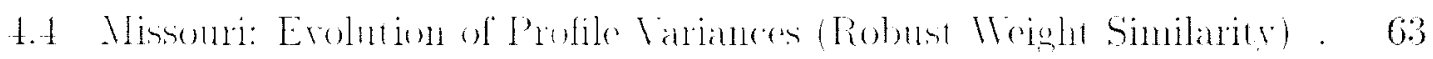

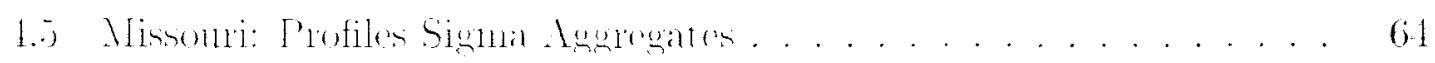

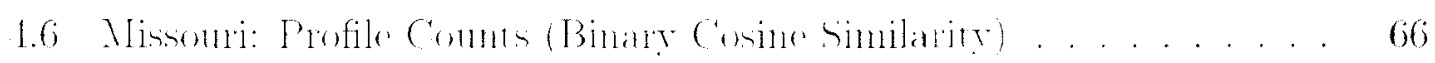

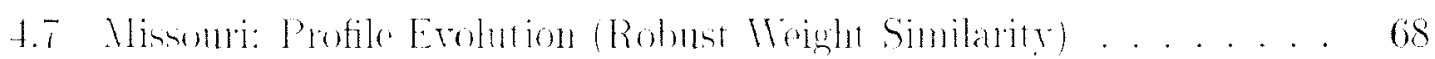

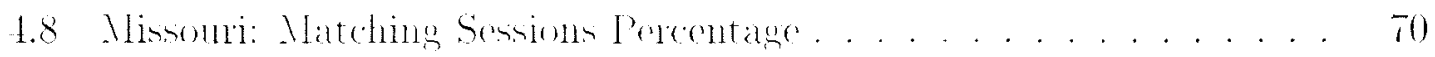

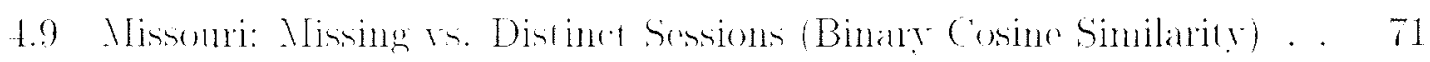

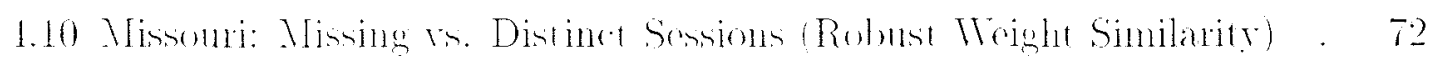

4.11 Missonti: Cardinality of Max and Min Sigma . . . . . . . . . Tt

1.12 Missondi: Erolution of Protile Cardinality Perentages (Cossim) . . T5

1.13 Minsonri: Evolurion of Profilo Cardinality Perentages (RobllT) . . T6

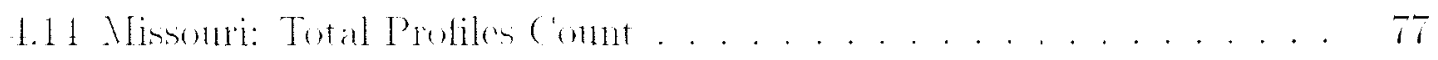

4.1.j Missomit Protilo Pair-wis Similarity Aggrouates. . . . . . . . T8

4.16 Missomit Evelution of Protile Densitios (Cossim) . . . . . . . . . 80

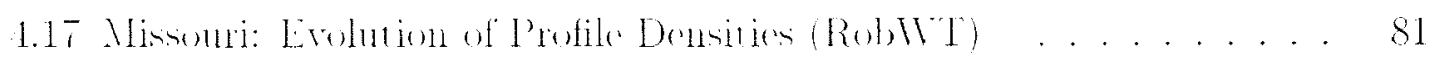

1.18 Missonti: Traditional Profilo Vintaners . . . . . . . . . . . 82

1.19 Missonri: Traditional Profilo Donsity . . . . . . . . . . . . . $8: 3$ 


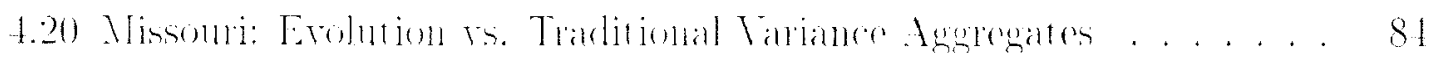

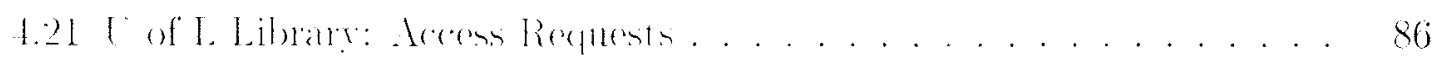

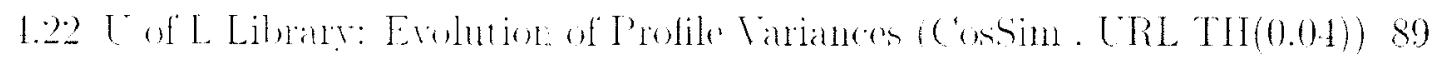

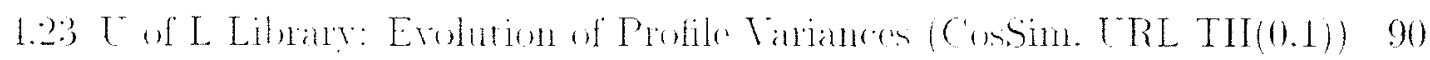

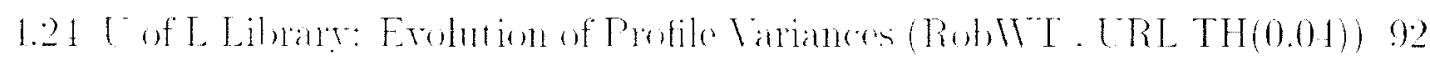

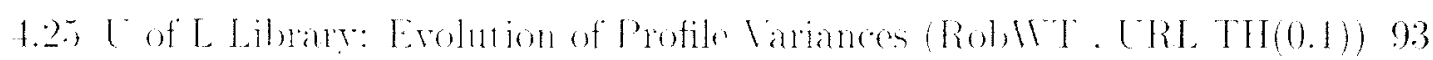

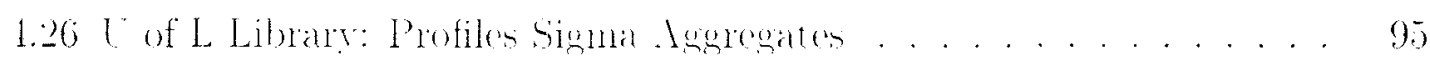

4.27 [ of L Library: I'rofile Counts (Binary Conine Similarits) . . . . . 96

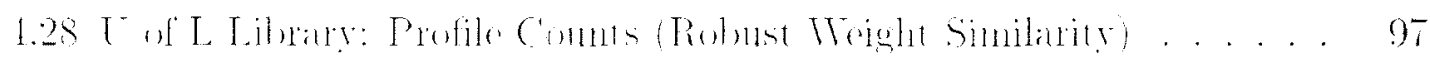

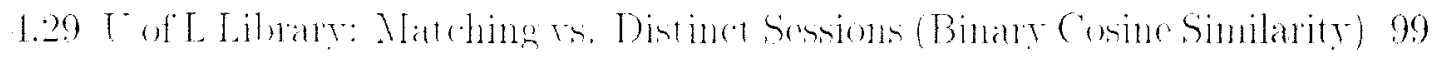

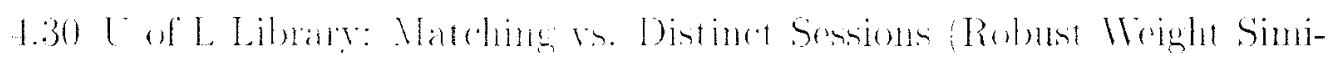
larity . . . . . . . . . . . . . . . . . . 100

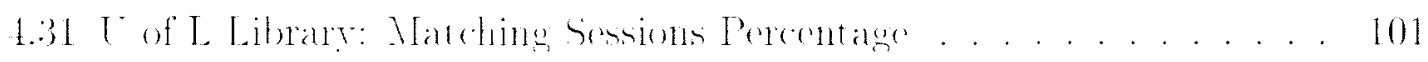

4.32 To of L Library: Cardinality of \ax and Win Sigma . . . . . . 102

1.33) [" of L Libary: Evolution of Profile Cardinalit! Pererntages (Cos.Sim) 10:3

1.31 [' of L Libary: Evolution of Profike Cardinality Perechatages (RohNT) 104

1.35 to of L Libary: Total Profikes Connt . . . . . . . . . . . . 10.5

1.36 T' of T. Library: Profile Pair-wion Similarity Aguregates . . . . . 106

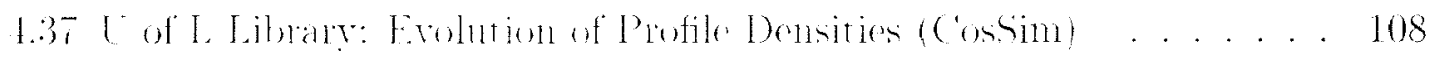

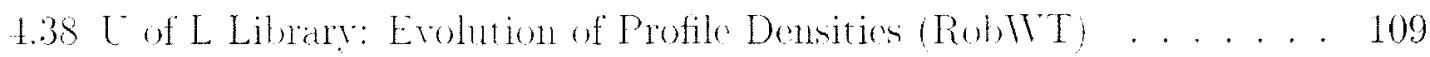

4.39 L of L Library: Traditional Profilo Variances. . . . . . . . . . 110

4.40 To of L Lihrary: Trarlitional Profile Density . . . . . . . . . . . . 111

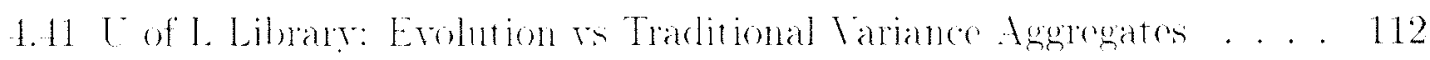




\section{CH.APTER 1}

\section{INTRODUCTION}

\subsection{Motivation}

The World Wide Web is the largest and most aceessible soure of information for both users and businessen. Organizations create their own wobsites to offer their services to the customers (individuals or other companies). and (anstomers flock to these websites to buy new books. rent musies. wad news. or perform any lind of business transaction. As more husinesses mored online. the compertion between businesses to keep their old colstomers and lure new customers has increased. since a competitor's wobsite is just one click away.

All these reasons have motivated online connanies to analyze the usage activity data that renults from online transations in order to better understand and satisfy their website users on an individual or group basis. for instane to support Customer Relationship . Managenent (CRY). Howerexe millions of clicks and online transactions

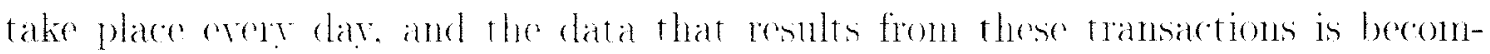
ing so huge that tring (o) use conventional analysis mothods is neither possible nor cost-effective, As a result. it has becone imperative to use antomated and effective methords to tum this raw data into knowledge that can help online organizations to better understand their users. 
Wob Lsage Mining is the precess of applying data mining tochnignes on web log data (transatetions) to extract the most interesting mode online artivity patterns and restract form then user profiles (c.g. a sot of LThLs) that ran be used in higher-

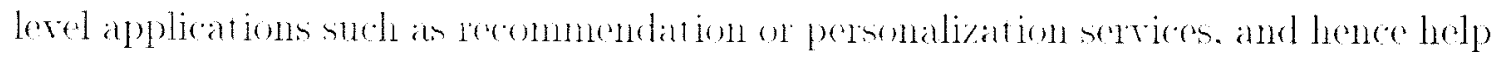
increase the online users sat isfaction and maintain their logalte

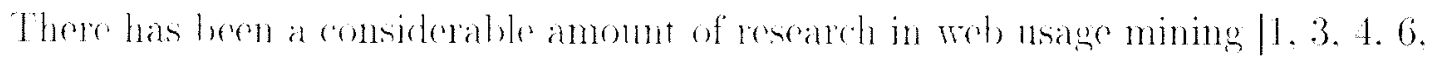

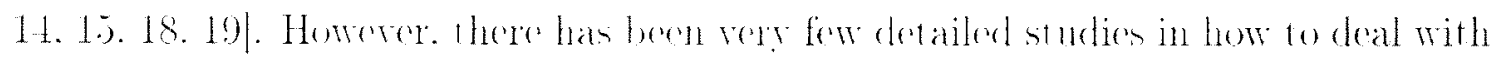

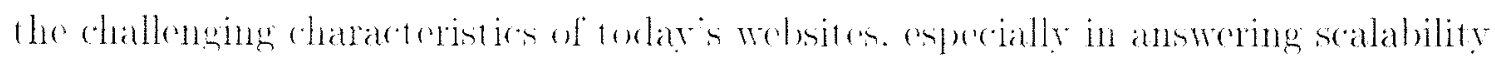

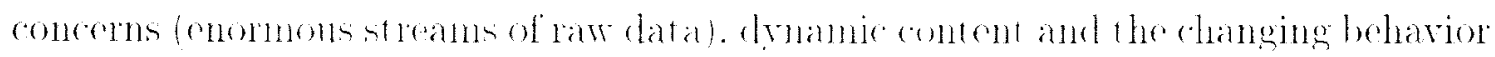
of users.

The content of most wobsites rhanges on a rogulat basis that ranges from monthly

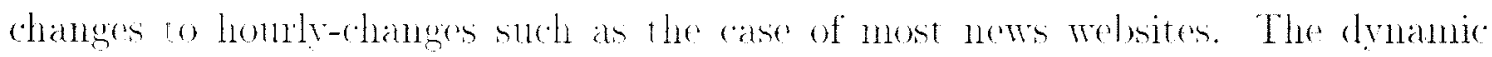
nature of wotsites content along with the social. oxononical. cultural. and other

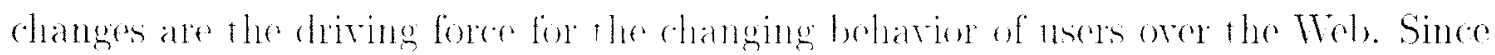

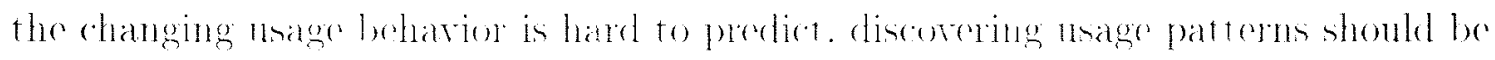
done dromanically and on a regnlar basis.

\subsection{Objectives}

This thesis proposes a new framernoth that can handle the changing content and

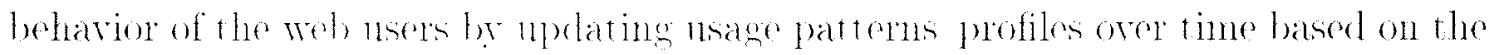
new wol, log data strom. In this fiamerork, wob usage mining is performed on a regular basis. such as weekly or daily on depending on the size of the logs, in case the logs anmot be loaded in memory in their entirely. It rach mun. the recent user artivition are compared against the existing profilen. then the profiles are upelated

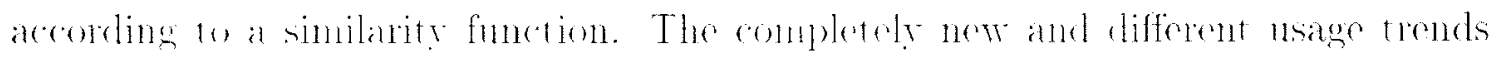

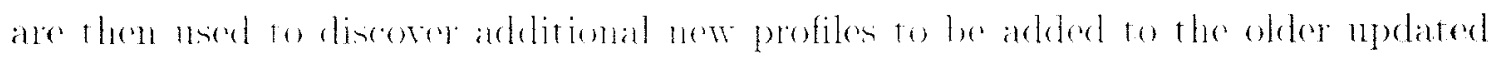
profiliss. 
Following this scalatse approath the wage patternes are expected to always be

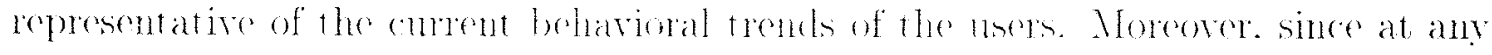
tine. only a snatler part of the new $\log$ data nudergoes the pattern discorery process. this appreath is efficient in termo of computational complexity and resource usage. These characteristics make one framertonk an efficiont and robust solution that can be embededed in a higher-lesel application that utilizes the profiles in intelligent wet) applications. such as to provide prediet ions for web page pre-fetching. load balancing. recommendations. or web personalization.

The objectires of this thesis an he smmmarized as follows:

- Handling the scalabiling of usagre pattern discovery from massire input web Ilsage data.

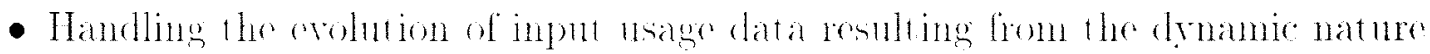
of the Wob content. and the changing beharion of whe nesers.

- Tracking the erolution of the discorered puefilen orer time by updating an archire of profiles in an on-going basis.

- Generating uner-friendly profile reprenentations that can be used in other higherlowel applications.

- Evaluating the culality of the propesere profile tracking. 


\subsection{Thesis Contribution}

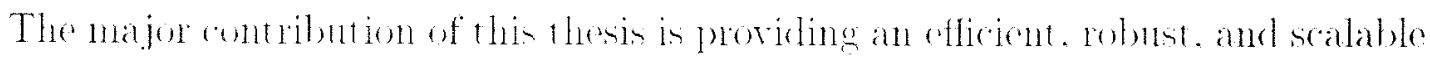
approach to discover the most interesting user beharion patterns on a welsite. This

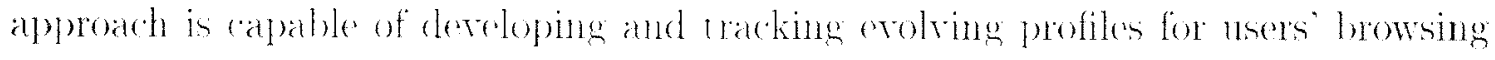
trouds on a single wobsite. These profiles ane petentially -at any given timo- the best

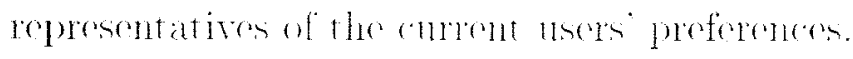

This thesis is also concenned with stulying and andyeging the crolution of patterns that are discorered. The approath adepted in this thesis mplates the pattems to the smallest detals. Hence observing their evolution will give an arenate pieture of how

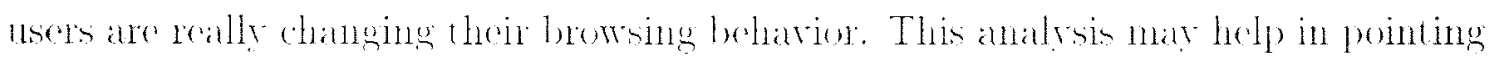

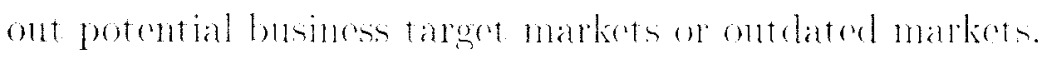

This thesis also sleggeste a maintonatues algorithm that will be applied periodically on the discosered patterns to enhaner their acculary and roliability. During maintenance. the patterns ate raluated. 1hus potentially allowing mely interesting

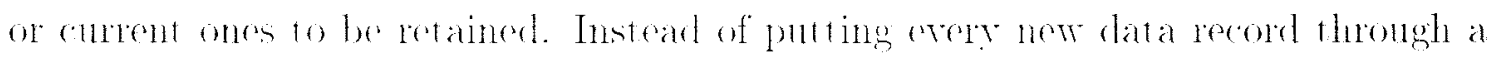
complete analysis. a large portion of data that already match the discorered profiles) will actually be disanded hased on appropriate similarity tests against these profiles.

Erenthomgh. one discossions are within the context of mining wob usage datal.

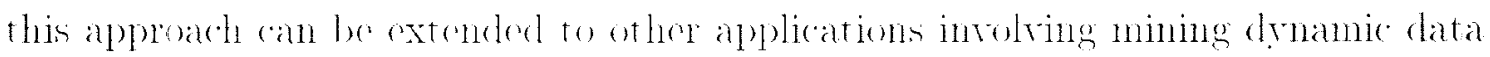
strams. such as mining and sndmmarizing varions ststen and notwork andit logs, or "ren trmporal text data sheh as bloger and online news.

\subsection{Summary}

The motiations behinel adopting the proposed franework are the need for andlyzing the wob users helavion on wobsites and the lack of mothods to handle changes efficiontly, while adhering to the sabability recturement of this task. We have also presented the main objertives and rontributions of this thesis. that is to develop a 


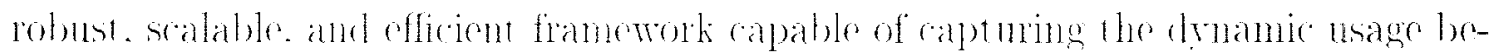
havion on the wat, as a set of erolving protiles. which conld be nsed in higher-fevel applications to moet a ratery of organizat ional gorals. 


\section{CHAPTER 2}

\section{LITERATLRE REY'IEI'}

\subsection{Overview of Web Usage Mining}

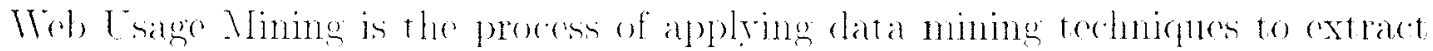
useful infermation unge patterne frem the wers log data. The analysis of the dis-

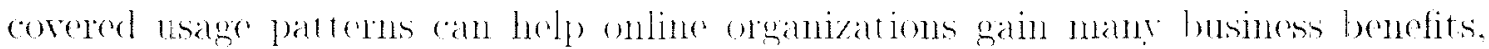
including:

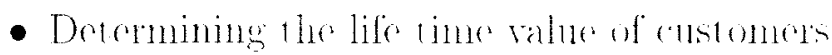

- Dereloping cors-product marketing strategies

- Enhancing puomotional anuparigns

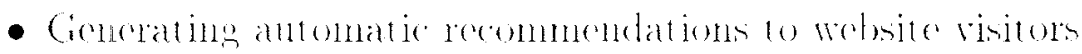

- Mile presonalization

- Efficiont organization of the rots sites content and structure

- Enhancing the server performance by prefetching pages that the user is most

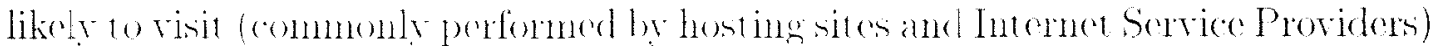


The abowe benefits ane mot limited to online stomes. but hore may also impare other

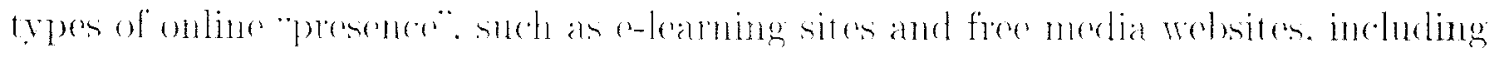
nors. publishers and olgital libarien.

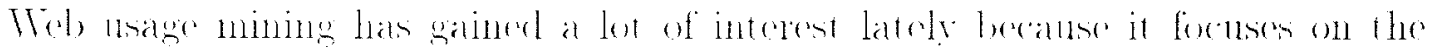

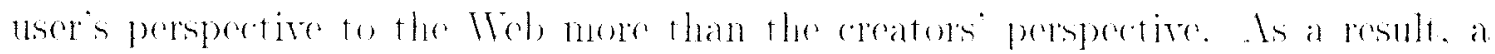

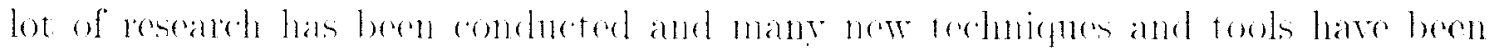

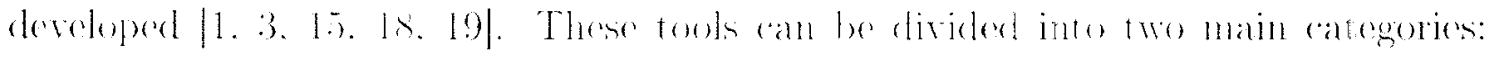

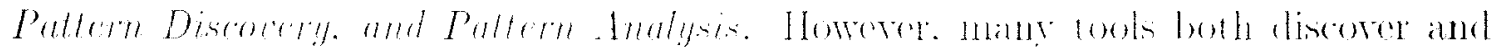

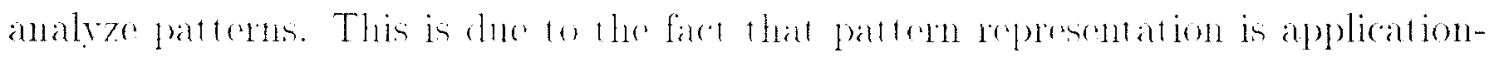

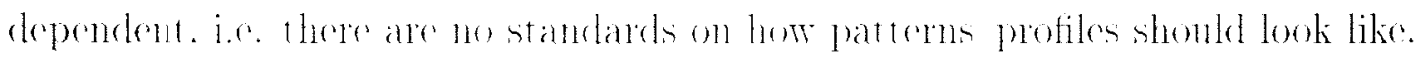

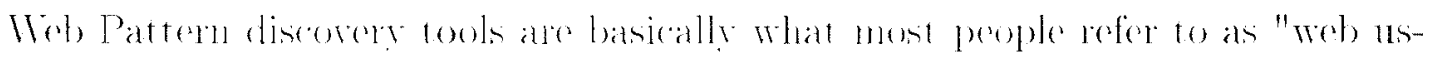
age mining" tools. They use many technigues from different disciplines inclueling

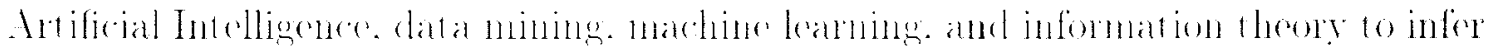

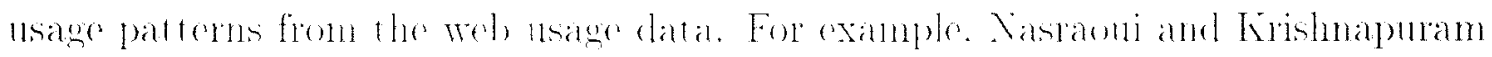

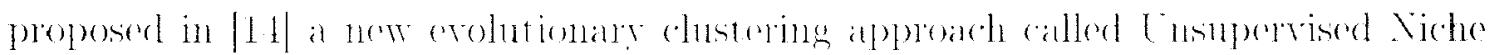

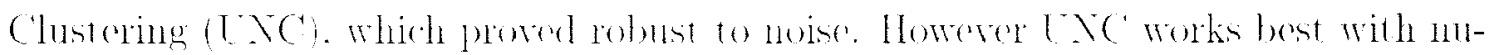

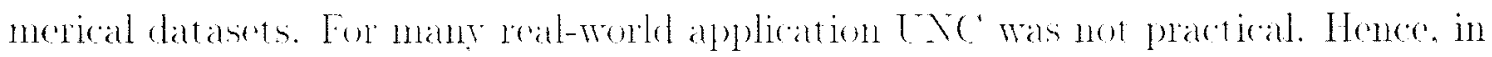

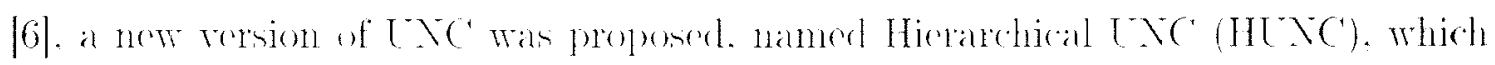

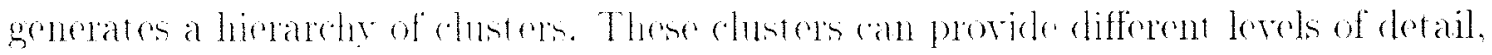
thus speoding up the mining process comsiderably. This thesis will provide a moditied

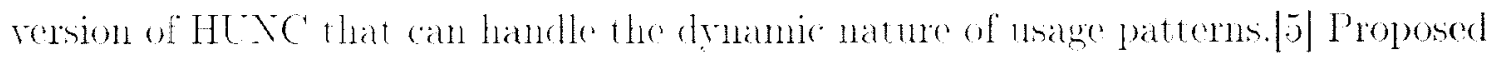
a complete framerwoth that automatically chasifies visitors to a wal site into different classes based on their navigation beharion. Once the visitor is rassified. the organization of links will be dynamieally (hanged on the-fly and links will be suggested to the user.

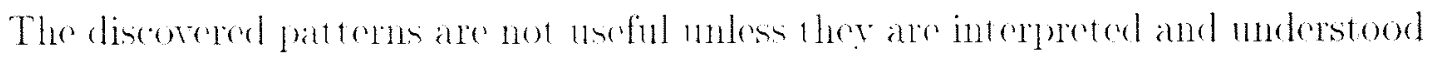
by the patern analysis teols. The interpretation of the patterns depend on the tool 


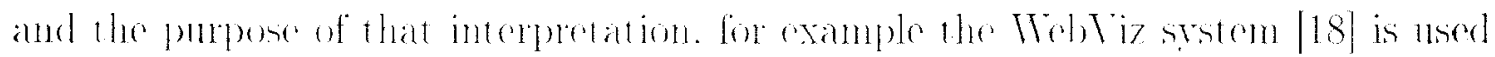

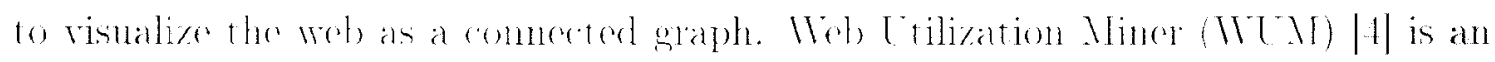

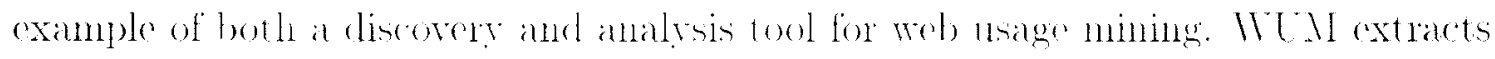

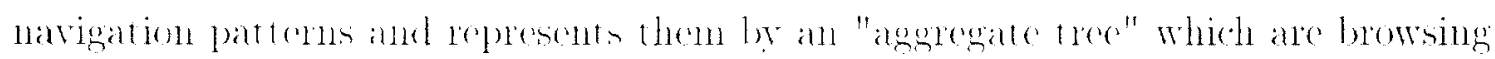
trails combined based on their common prefix. Noroorer. WT I offors an SQL-based language called MINT that enables the human expent to fommat the ontput and choose the interestingness clitoriat.

\subsection{Sources of Web Usage Data}

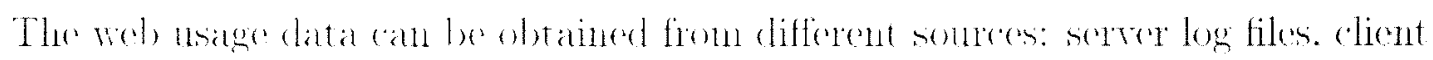
side data and woh proxy logs. The most innertant somer is the server log files since they explicity record the clickstreans for all the usess on a particulat wolsite and they are moleh easien to obtain since they are onned be the organization that owns the wall servers.

The information arailate in log files inchude the dent s IP address. time of acess. pages risited. provions page risited and other folds. Howrerer. in its raw forme the

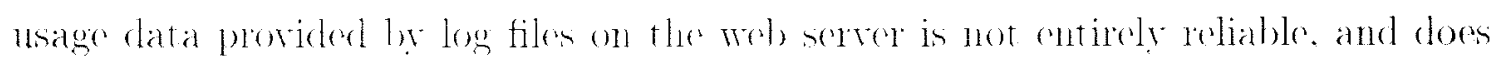
not reftert the actual browsing behavion due to the mious lewe of eaching on the

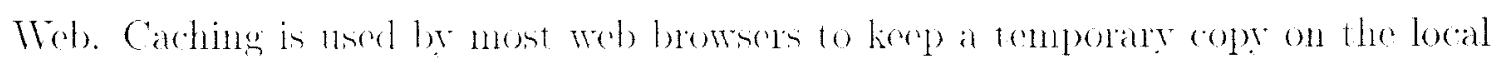
machine of most recently visited pages. under the assumption that these pages would

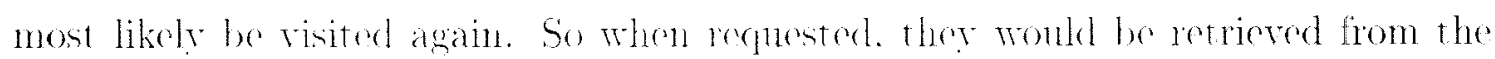
temporary location on the local machine to roduce both the loading time of those pages and the netronk trallic loarl. Is a renult. all the cached pages are not recuested from the server. Thess. they are not reotrided in the server log file.

Other information that wan he fonnd on the sereser include query data which is the set of kerwords that the users search for within that wobsite. as well as cookies 
which are small files stored on the local machine and that can be accessed only by the wob serress in a sperial list stored in the cookic. The purpone of cookies is to heep tractiof aners because of the linitation of the stateless comection model of the HTTP

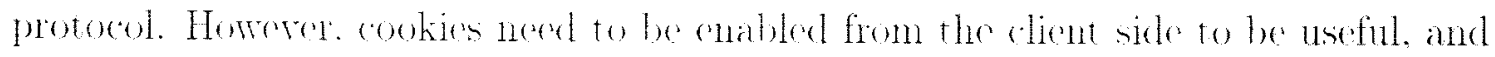
they also laise coutain privacr concerns.

On the elient side data can be collected using client-side scripts such as Java Seript and Java Applets. Howerer there is a trade-off between the additional computational overhead (such as in Jara Applets) and the anome of data collected fonch as the sheer amount that Jara Seriph can collect compared to Java Applets). Client-side collertion of data solves the problem of caching since the seript is mubeded in the page itself not on the serror side. so erery time the page is aceessed - even if it is cached - the data will be collected and sent to the servel. Hownere as in cookies,

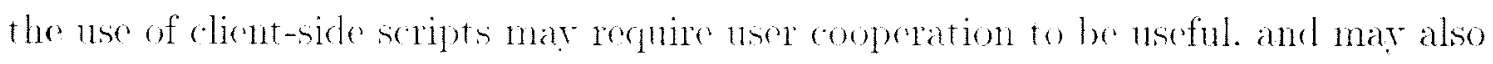
raise some privacy concerns.

Another somere of nago data is the weh proxy servert. A web proxy is basically a server midway between the clime and the serere. that caches the most recenty requested pages in order to reduer the hading time of wol pages and reduce the network traffic load. It hlatres the same concept of eaching be wob browsers on local marhines. howerer a wels proxy is typically shared by mult iple users and caches pages from multiple wob servers. Hence a web proxy server can provile aceurate and rich informat ion about the common incrents of multiple users on multiple webstes, which makes it a valuable source for the wel wage mining process. On the of her hand. local (cliont-side) raching provides information about only a single user. Howerer. obtaining the data from woly proxien is hatel and raises mom privacy concents, because wels proxies contain the activity of multiple web servers.

The experiments done in this researele are based only on web usage data collected from the web serer. Hownor. the framerwh that is presented can easily be extended 
to process usage data collected fiom the client side and prosy servers.

\subsection{Pattern discovery process}

Analyzing how nows aceses a weste can be critical to the organization as discussed carlier. and an be one of the main faceors in detemnining the organization's

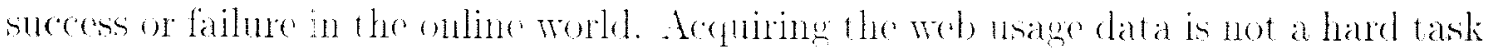
since most of the data is on the wets servers ormed he this oreanization. Howerer. before this raw data is avalable for processing he the patton discorery algorithms. it needs to be prepared preprocessed for it to be nosful.

\subsubsection{Preprocessing usage data}

Preprocessing the usage data is not a trivial task |1. 2. 19|: it can be arguably the most diflicule task in the Meb Eage Mining process due to the incompleteness and complexity of data. It is also one of the nost important tastis in wob usage mining

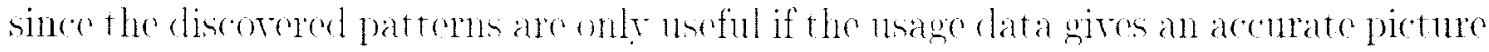
of the user areesses to the wel, site. There are two primary tasks in preprocessing: data cleanimg. and transaction identification also known as sessiomiantion.

\section{Data Cleaning}

During this process. all irrelevant items are climinated from the server log. An imelerant item conlel be an acess to a piotne. a clip). or anything that is not of any

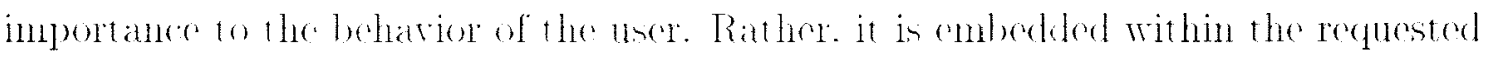

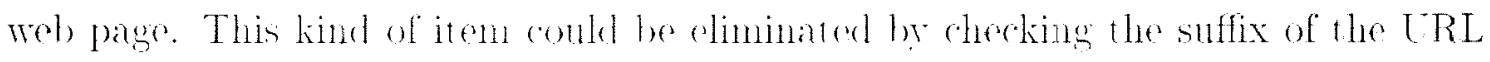
name and romoring all ontries oncling with CiIF. JPEG...otc.

Another kind of imelerant item comes from repuosts originating from web agents like search engine bots. crawlers. of spiders. Becanse thene agents access the website

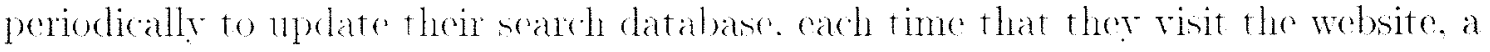
new entry is creared in the log. Set this does not represent an actual nuser. Hence. 
they shomk be remored. One way to do that is be checking for some known IP addesses or crawler identifies. and then romoving all the comresponding ontries from the $\log$.

\section{Transaction Identification}

The sequences of page requestes must be gromped into legical muits to bo used in the mining process. Each of these logical units is called a sescom which is the set of pages that are risited be a single user within a predefind period of time. This step

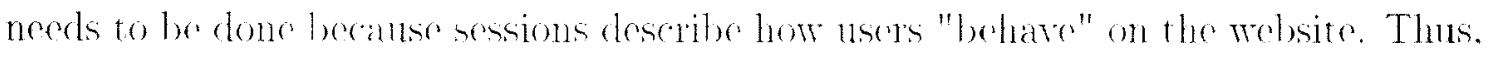
individual TRE records aro meaningless. This might sems like a trivial process. howerer. some issmes need to be adrliesserl.

One issme is how to determine that the areess reconds really belong to a single user. The problem is that not erey single IP address represents exactly one wser, and this is due to the fact that orelinary users (nomally) do not own a universally unique IP address because of the high cos and low availability of IT addresses. So Internet Service Providers (ISP) om one on more IP addresses and use them to comect their clients wo the Intoret. ISP' typically hare a poot of prexy sorers that aro used to speed up accessing the Interne by multiple clients. so all these aceesses will be seen on the log file as reming from one IP adderes hence comsidered one user. Also. some ISPs randomly assign each recuest from a single user to a different IP address for privary and optinization purpeses (IP address rotation). Which moans that multiple IP addresses on the log file may actually ropresent a singlo more. Moreorer. a user accessing 1he wotsite fiom different machines may appeat as multiple nows on the sireresirle.

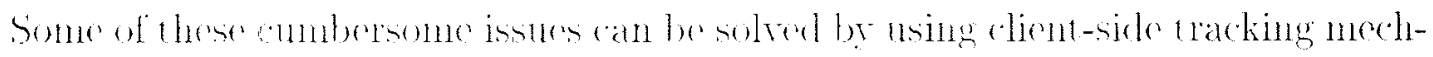

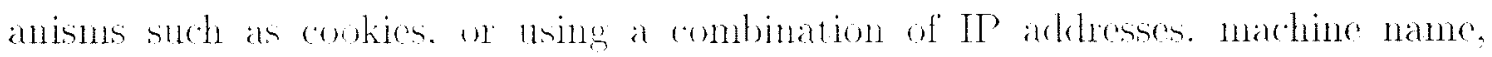
brower agent. and other infomation. Howner. cliont-sikte tracking meds to bo renabled by the rlent himsolf. and using a combination of different data (such as IP 


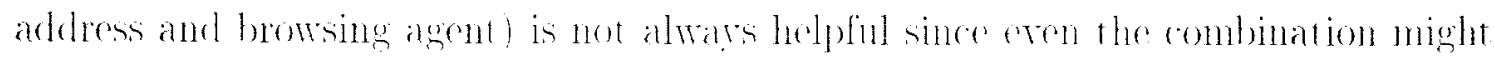

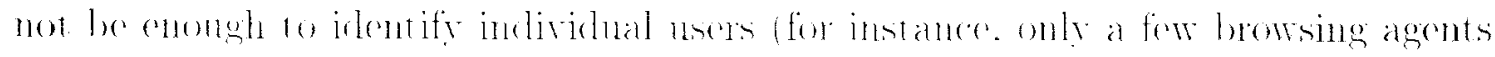
like Interne Exploter and Mozilla Firefox dominate the rest).

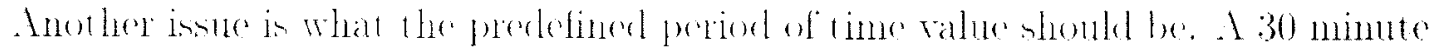
timeout is often nised based on the results of $\mid 1$. like online somes howerer it might not he the hest ralue for nerts or publication

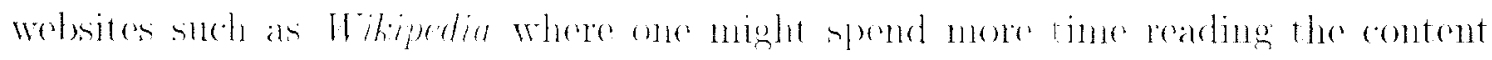
rompared to online store.

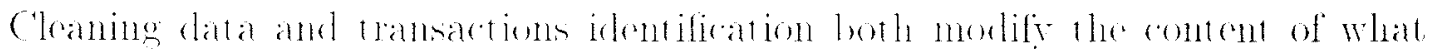

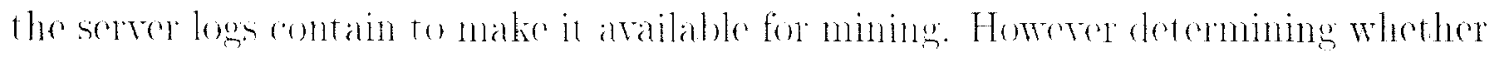

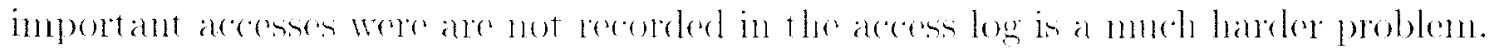
The main reason for the absenee of these records is carching.

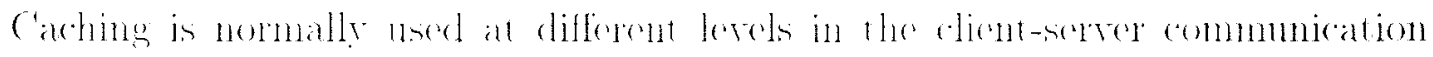

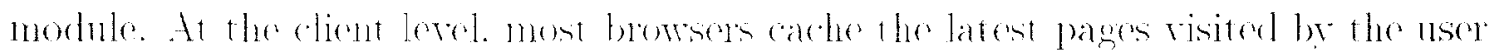
muder the assumption that thene pages will be risited again. Carhing also happens on the proxy sorres of the ISP. Where data reduested from multiple whers is stored temperanily: Caching helps in redueing page loading time on the client side and

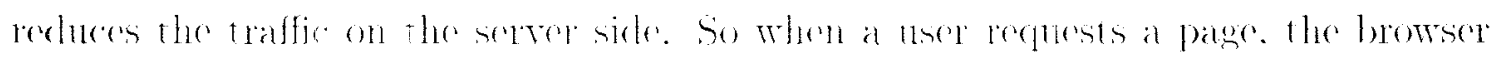
searele for it in the carhe first. if it is not there then it will reguest it from the server. which also chechs the prosy server first for cached data before going to the website

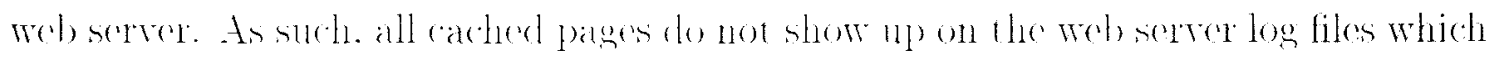

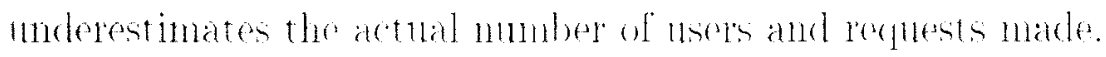

To orercome this problem chent-side scripts such as Java Seript and Jara Applets an be nsed. since there are ombetded in the page itself. Whenever the page is accessed -eren if it in cached-the data will be collected. Another solution is to access

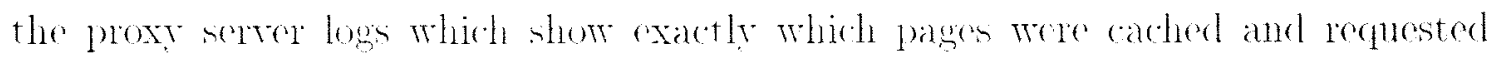
again. Howerey. He first appruach noeds the compliance of the wsers. and the second 


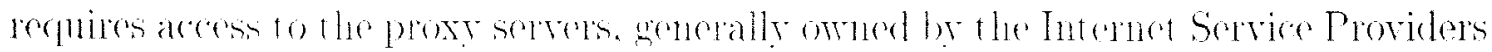
(ISP). which an mise major privary issmen.

\subsubsection{Pattern Discovery}

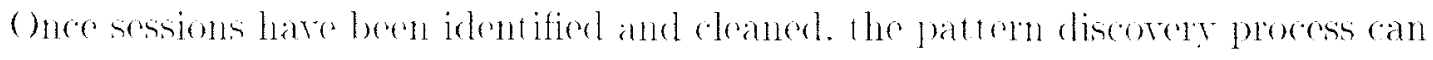
begin. There are seremal methods that can be used to diseorer interesting patterus,

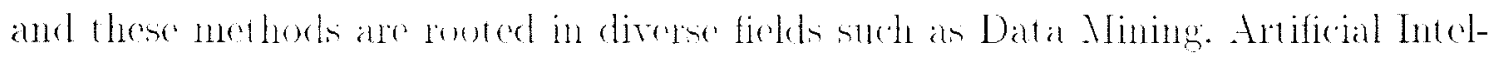
ligener. Statistics. Nachino Learning. and Pantern Rerognition.

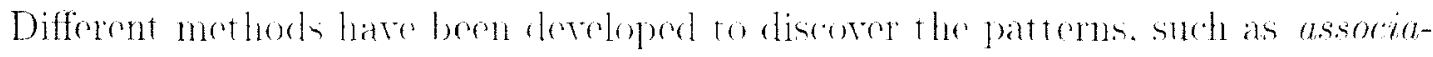

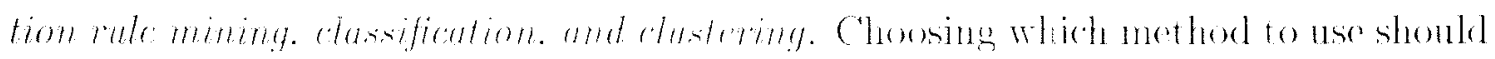
take into consideration any prion knowlerge of the Mol, Data. Fon example in associ-

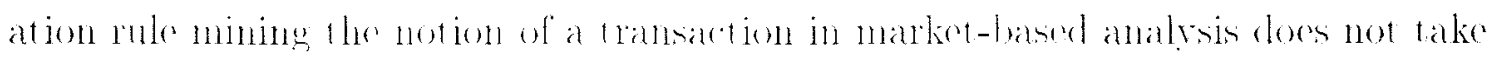

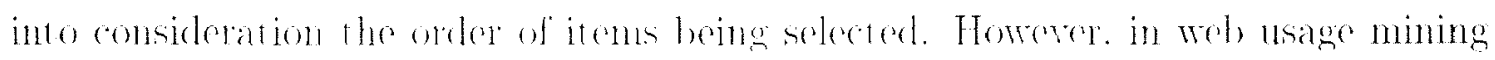
the order of pagen recenested in the session may be innportant siner it reflerets how the user actually reacherl a spereific page of interest. Moreorer. prion knowledge about tho data may help in determining some of the parameters like the defanlt timeout period discressed arlion.

\section{- Statistical Analysis}

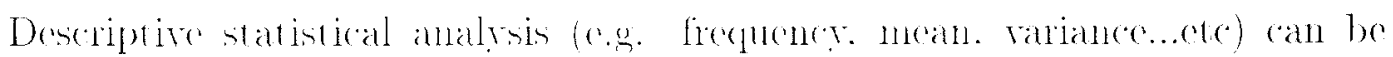

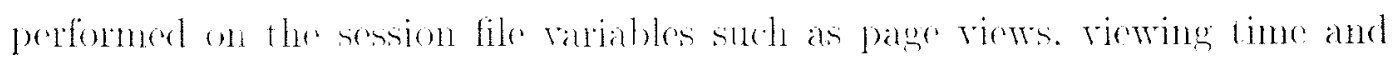
longth of navigational pathe. The ontput of applying statistical methods conde

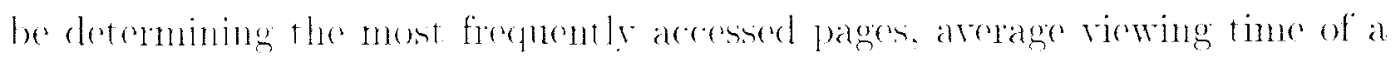
page. arerage length of marigation pathe to a sperifie page or the most common

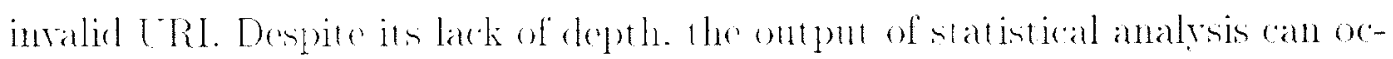

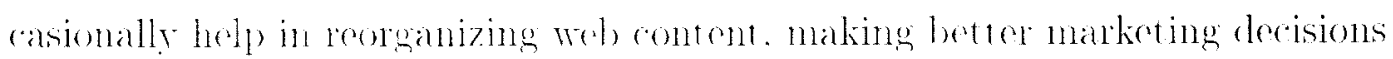
and enhancing ststom performance and security. 


\section{- Sequential Pattern}

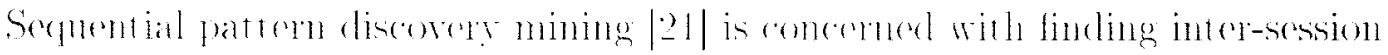
patterns such that the presenes of a sot of iteme is followed by another item

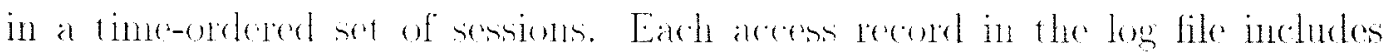
the time of acesss se that when preprocessing the data these timestamps will be atrathed to their sessions. Discoreving the sequential patterns allows the organization to prediet the lisers next click, wheh is rital infomation to tailor

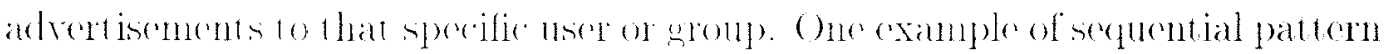

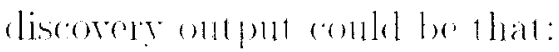

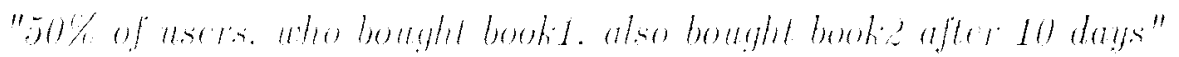

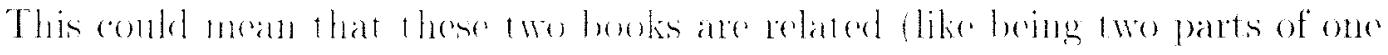

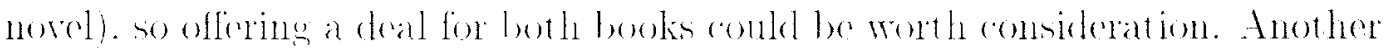

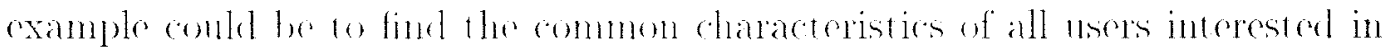
"Bookl" in thr prepiod "May 1-1- May 15th".

\section{- Path Analysis}

Path analysis mothods |20| are concenned with representing the wolsite as a

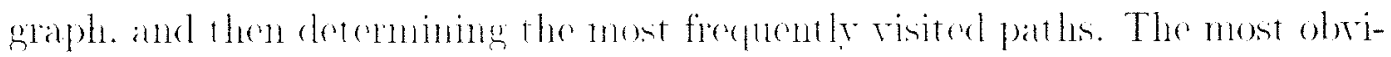

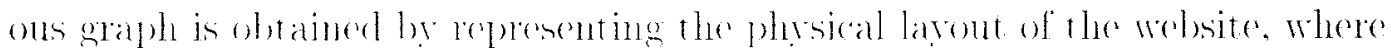

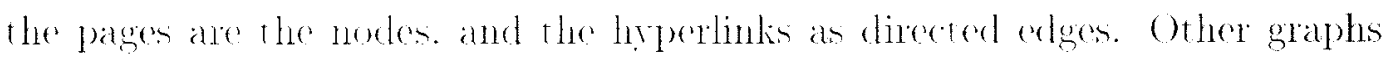
may represent the alges as the mumber of users going linom one page to another or the sindianity sueh as in [2:3]. or using Narkor Chains [25]. or using special tric-like struetures such as the WAP-tren $|26|$. An example of the output of path analyis contel be:

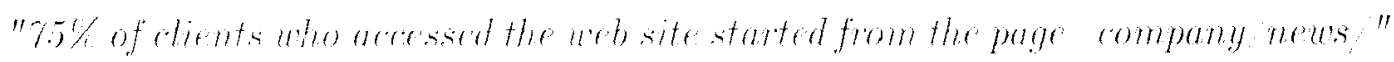
This moane that the page compm! mens. is the first page that most visitors 
acess. Hence. making sure that it links to all other payes might he a good idea.

\section{- Association Rule Mining}

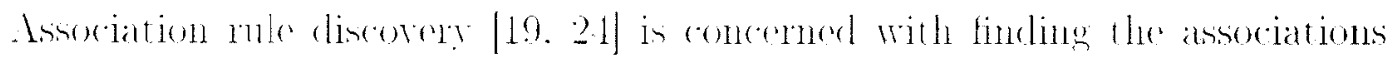

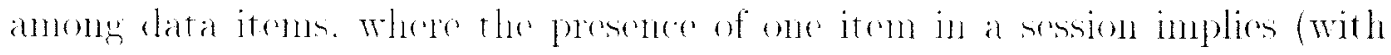

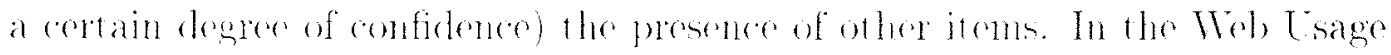
Mining context. this reters to the sot of pages aceessed together by a single user.

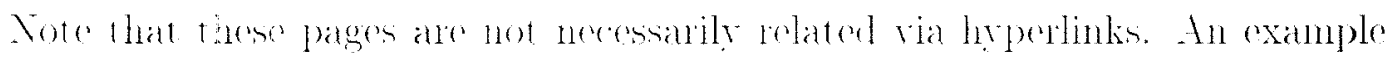
of an associat ion rule might be:

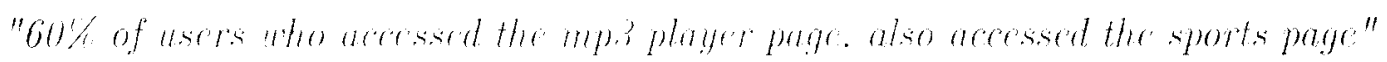
This means that both pages are related. eren thomeh they probably are not linked via hyperdinks since they ane in different departments. so this indicates the nead to hawe hyperlinks between the two pages.

\section{- Classification}

Classification is the process of mapping a lata item into one of several pre-

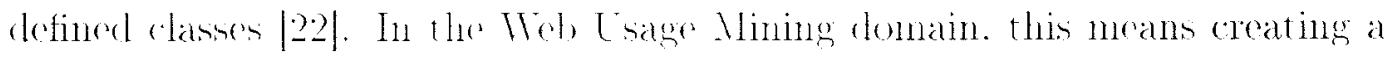

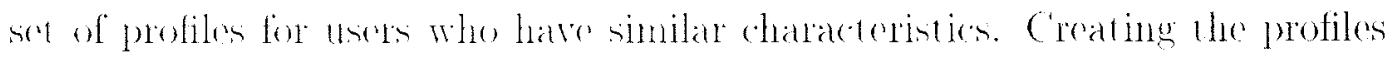

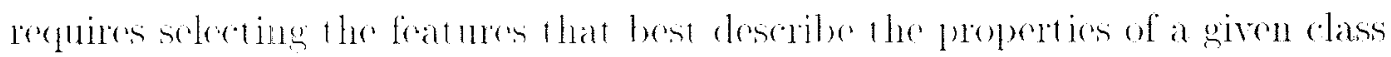
or catreory: These foatmes conle be demographical information such as age.

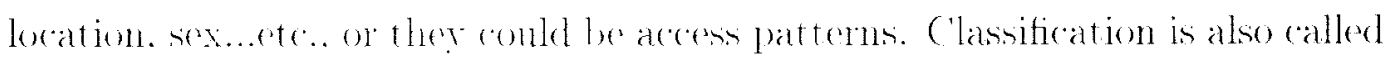
Superised Leaming since the dasses ane hinow in advanec. An example of a dassification rulo (omlil lex:

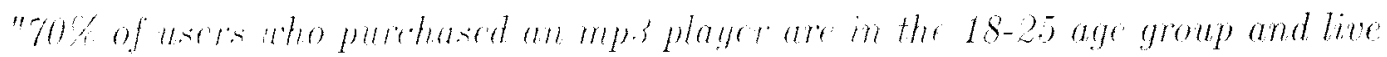
in the Enst Coust"

This mo suggests that areating a suredial offer for all users between 18-25 olds 
who lixe in the East Coant might be a goos plan.

\section{- Clustering}

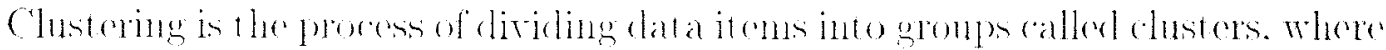
all the itome in one chuter are more "sinilar" to areh other than any oflece

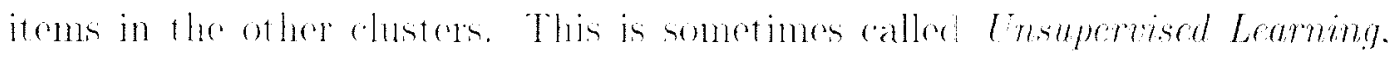

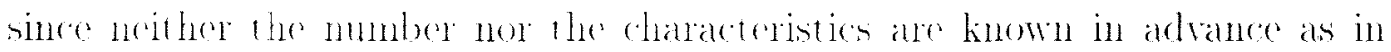

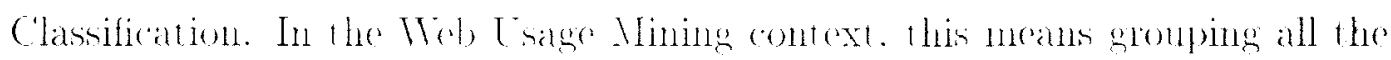

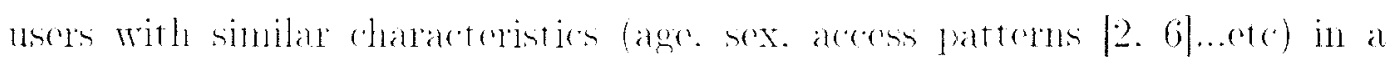
single cluster. Clustering ran facilitate the derelopment and execution of future marketing stategies. such as dranumally dhanging the content of a website

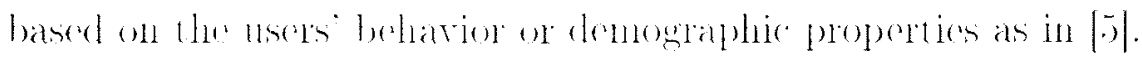

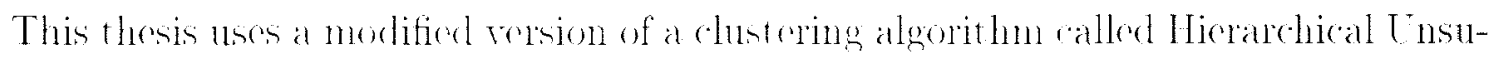

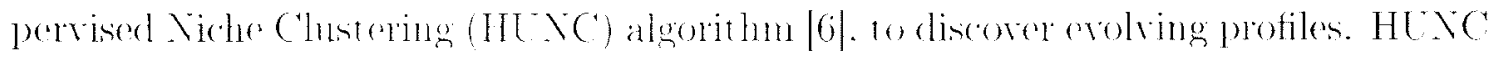
is discussed in Section 2 . 1 .

\subsubsection{Patterns Analysis}

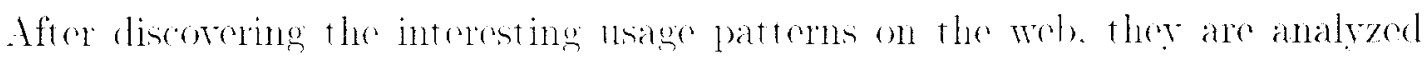
with specialized rools a boter muderstand them. The analysis wook are a mix of different fiolds including statistion. gapheses. visnalization and database querving.

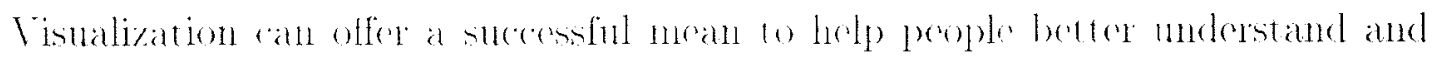

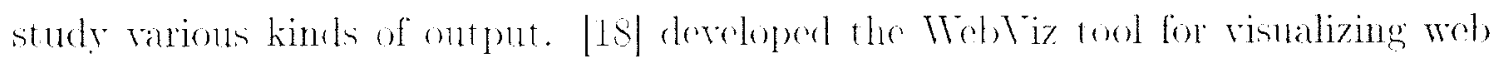
aress patterns. The Nob is represented as a dimened graph whore nodes ane the pages and edges are the hyperlinks. The areess patterns are nised to formulate a wob 
pathe and the une of the fool and analyze any portion of the web site and see how nors anc moving from one page to another.

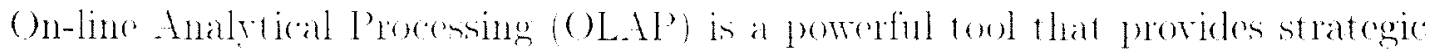

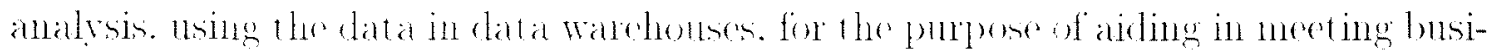

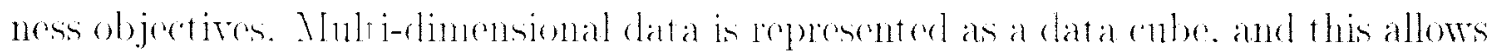
the noar instantaneous analysio and display of large amomuts of data. Areoss logs

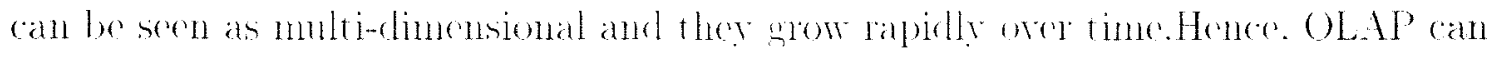
be applied to better analyze and numlestand usage patterns. An SQL-like querying mechanism has been proposed for ITEBMIJVER [19]. and it provides a simple way to extract information about ascociation rules. For exandele the guery

\section{SELECT association-rules $(A * B *)$}

FROM $\log$

WHERE date $>=01 / 01 / 07$ and domain $=$ "com" and support $=2.0$

will extract all asocedation rules in the".com" domain. that ane after Jan 1 st 2007.

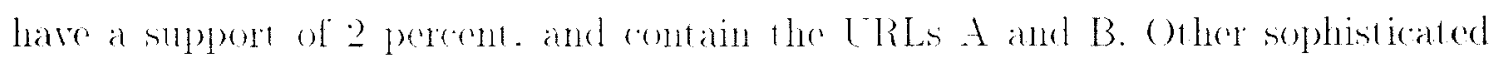
patern analyis fools have hoen proposed. such as XINT in [26].

\subsection{HUNC}

HLXC is a herarchical resion of the Tusupervised Niche (hustering (CXC) al-

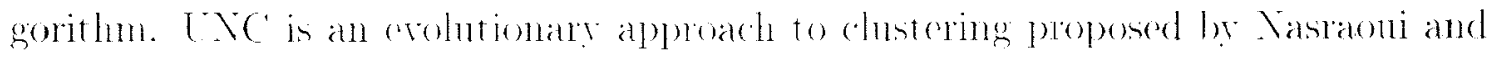

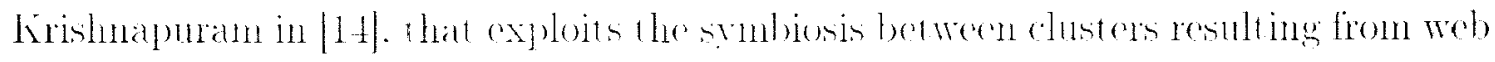
usage mining and genetic bologieal niches in nature. LY uses a Genetie Algorithm (G.1) [16] to avolwe a population of candidate solutions (user profiles) throngh gener-

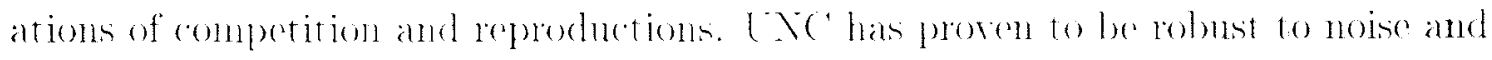
makes no assumptions about the mumber of clusters. Howerer. IC Mas formulated based on an Enclidean metrie spare representation of the data. 
Table 2.1: Log File Sample

\begin{tabular}{|c|c|c|c|c|c|}
\hline IP Address & Time & Method/URL/Protocol & Status & Size & Agent \\
\hline 67.99 .16 .111 & {$[01:$ Jan $2007: 00: 20:(15-0000]$} & 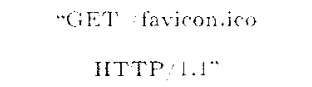 & 404 & 296 & $\begin{array}{l}\text { "Nozilla/5o (compati- } \\
\text { ble:Google Desklop)" }\end{array}$ \\
\hline $65.54 .1 \times 8.110$ & [01 Jan $2007: 00: 56445-0500]$ & $\begin{array}{l}\text { "GET roberts.txt } \\
\text { IITTP. } 1.0\end{array}$ & 40.1 & 283 & $\begin{array}{l}\text { "msubot/t.0 (- https/search. } \\
\text { msn,con/mstrbot.htmin" }\end{array}$ \\
\hline 65.11 .237 .191 & {$[01: J a n / 2007: 01: 01: 15-0500]$} & $\begin{array}{l}\text { "CiET rjmil- } \\
\text { lol fme txt fmissog.lotml } \\
\text { HTTP 1.]" }\end{array}$ & 401 & 311 & $\begin{array}{c}\text { "Mozilla } 4.0 \text { compatiblemsIE } 6.0 \\
\text { Windows NT } 0.1 \text { : SVL" }\end{array}$ \\
\hline 74.6 .131 .201 & {$\left[01, J_{i+11} \quad 2007: 01: 55: 53-0500\right]$} & $\begin{array}{l}\text { "GEL industrial } \\
\text { ITTP, } 1.0 "\end{array}$ & 200 & 018 & 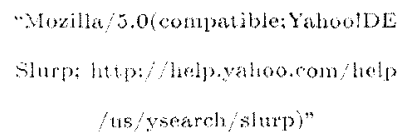 \\
\hline 74.63 .131 .201 & [01 Tan $2007: 01: 5,5,50-0500]$ & $\begin{array}{l}\text { "GET indus- } \\
\text { trial left } 2 . h \mathrm{~m} \\
\text { HTTP: } 1.0 "\end{array}$ & 200 & 8.371 & 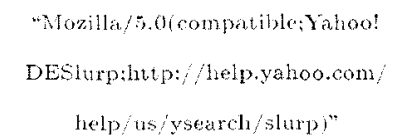 \\
\hline
\end{tabular}

HUNC generates a hierarchy of clusters which gives more insight into the Wob Mining process, and makes it more efficient in terms of speed. HUNC does not need to know the number of clusters in adrance like in most clustering algorithms (e.g. KMcans), can provide profiles to match any desired level of detail, and requires no analytical derivation of the prototypes. Noreover, HCNC calculates the similarities between web pages based only on the user access patterns rather than content.

\subsubsection{Preprocessing the web log file to extract user sessions}

The access log file is the raw data used to discover the user patterns/profiles. Each visit to a web page by each user is stored as a log entry; each log entry consists of information about that particular access such as the access time, IP address, URL page. etc. Table 2.1 shows an example of such a file. 


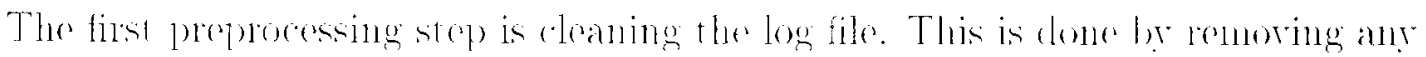

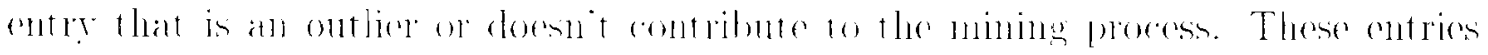

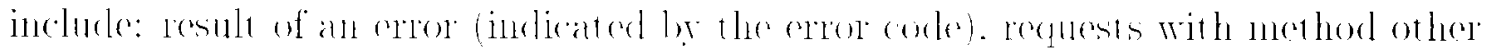

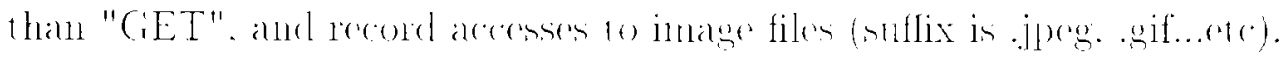

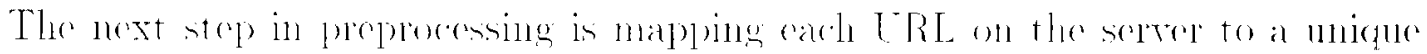

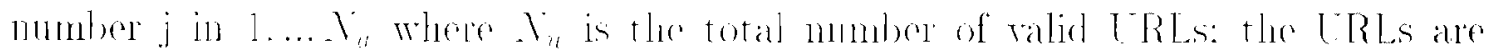

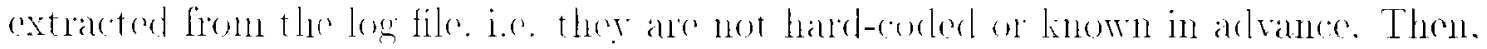

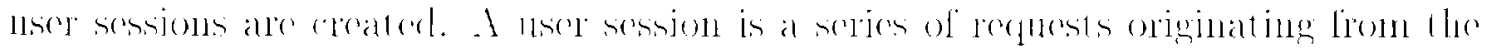

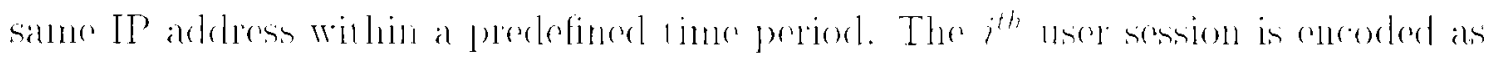

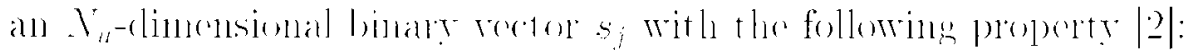

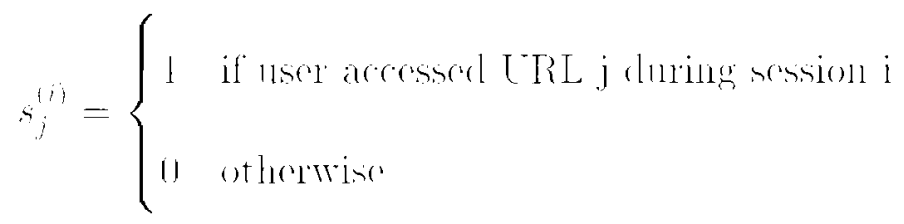

The set of all sessions is donoterl as S.

\subsubsection{Assessing Web User Session Similarity}

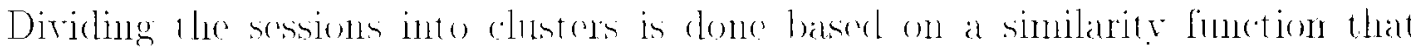

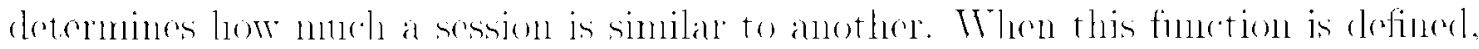

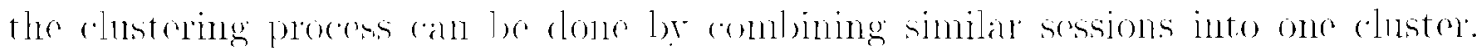

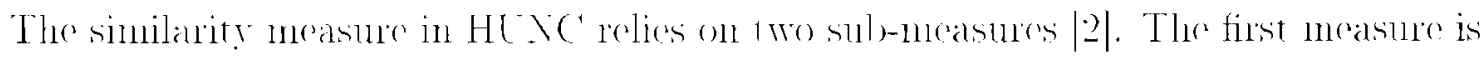
girolis lex:

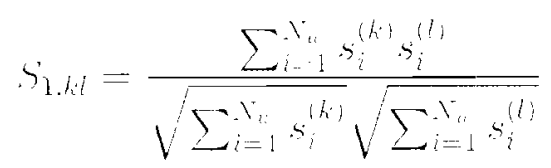

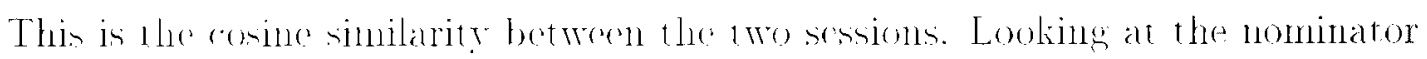

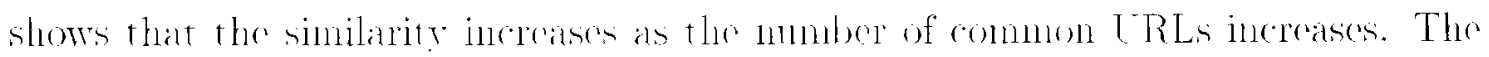




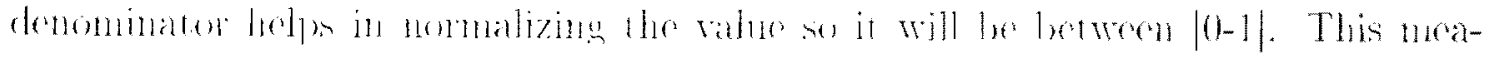

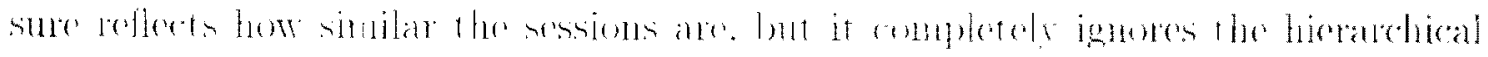

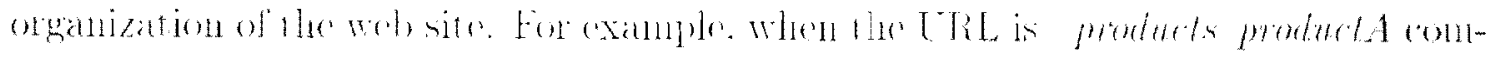

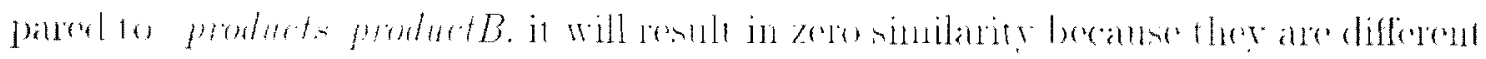

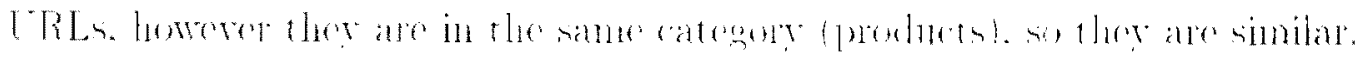

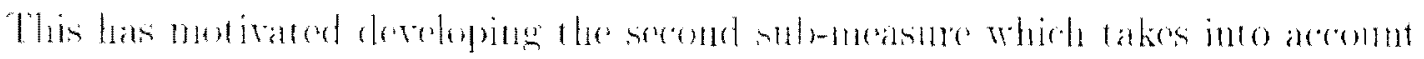

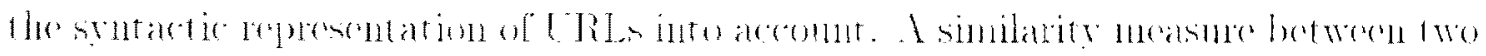
LRLs was dolined as follows |2|:

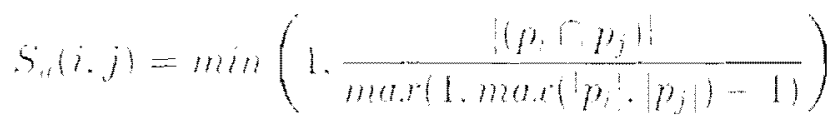

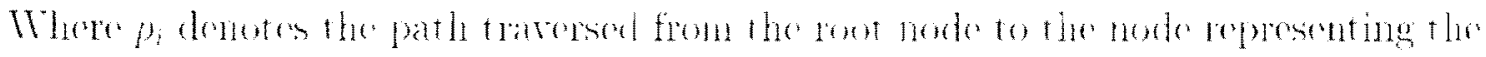

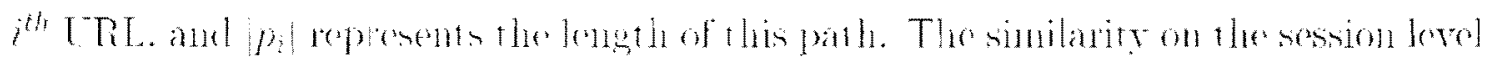

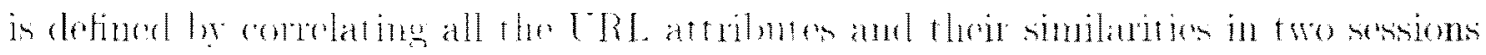
at follows:

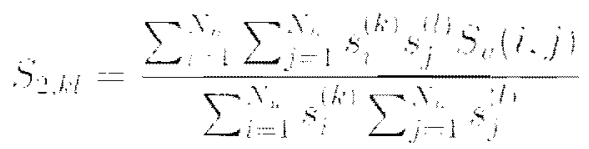

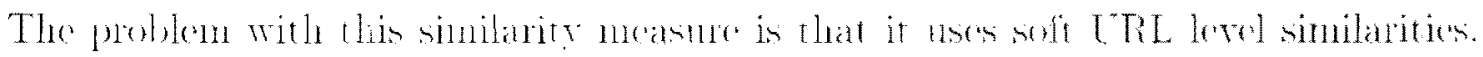

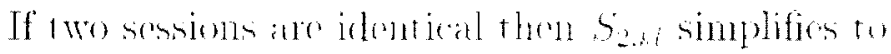

$$
S_{2, H}=\frac{1}{\sum_{i=1}^{i_{i} s_{i}^{(h)}}}
$$

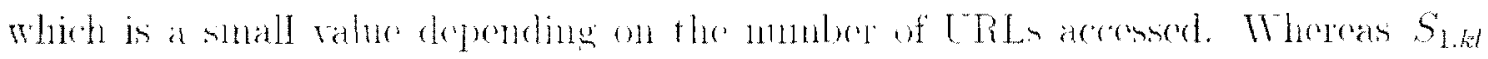

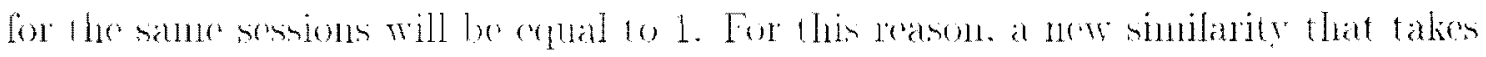

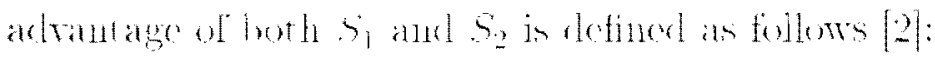

$$
S_{n+2}=m a n\left(S_{1,1,} \cdot S_{2,4}\right)
$$




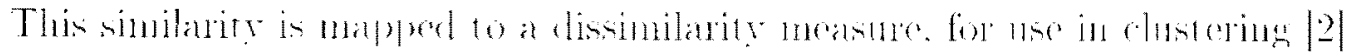

$$
H_{3}^{2}(h)=(1-S)^{2}
$$

\subsubsection{The HUNC Algorithm}

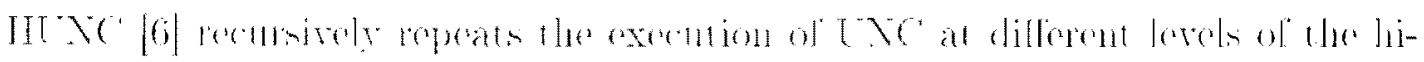

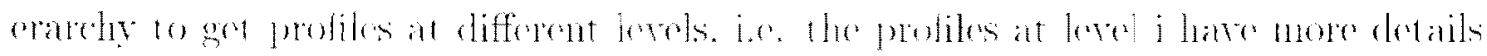
than the protiles at lored j-1.

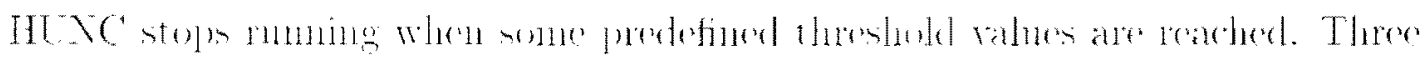

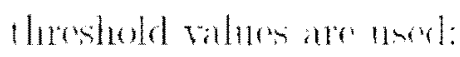

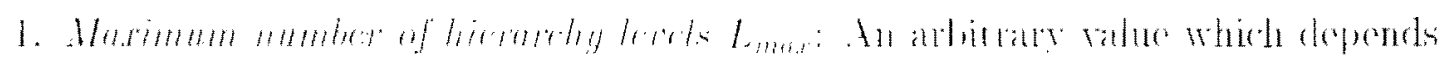

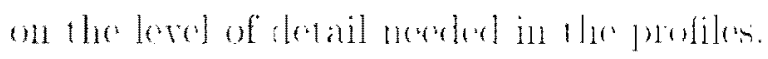

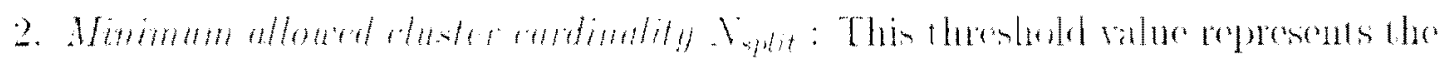

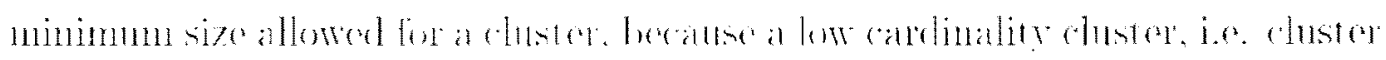
with small mumber of CRIs. maty not he interoming.

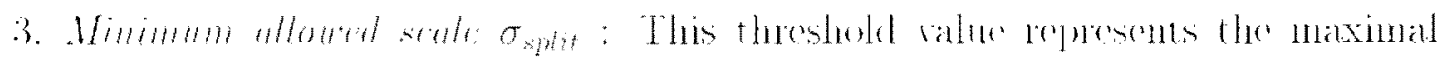

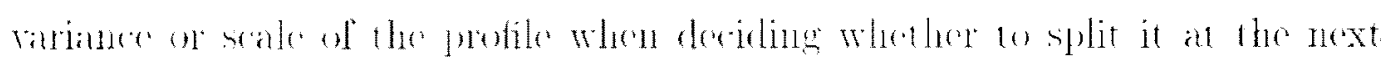

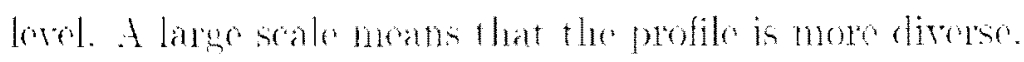

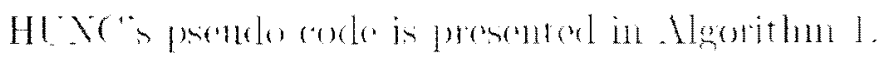




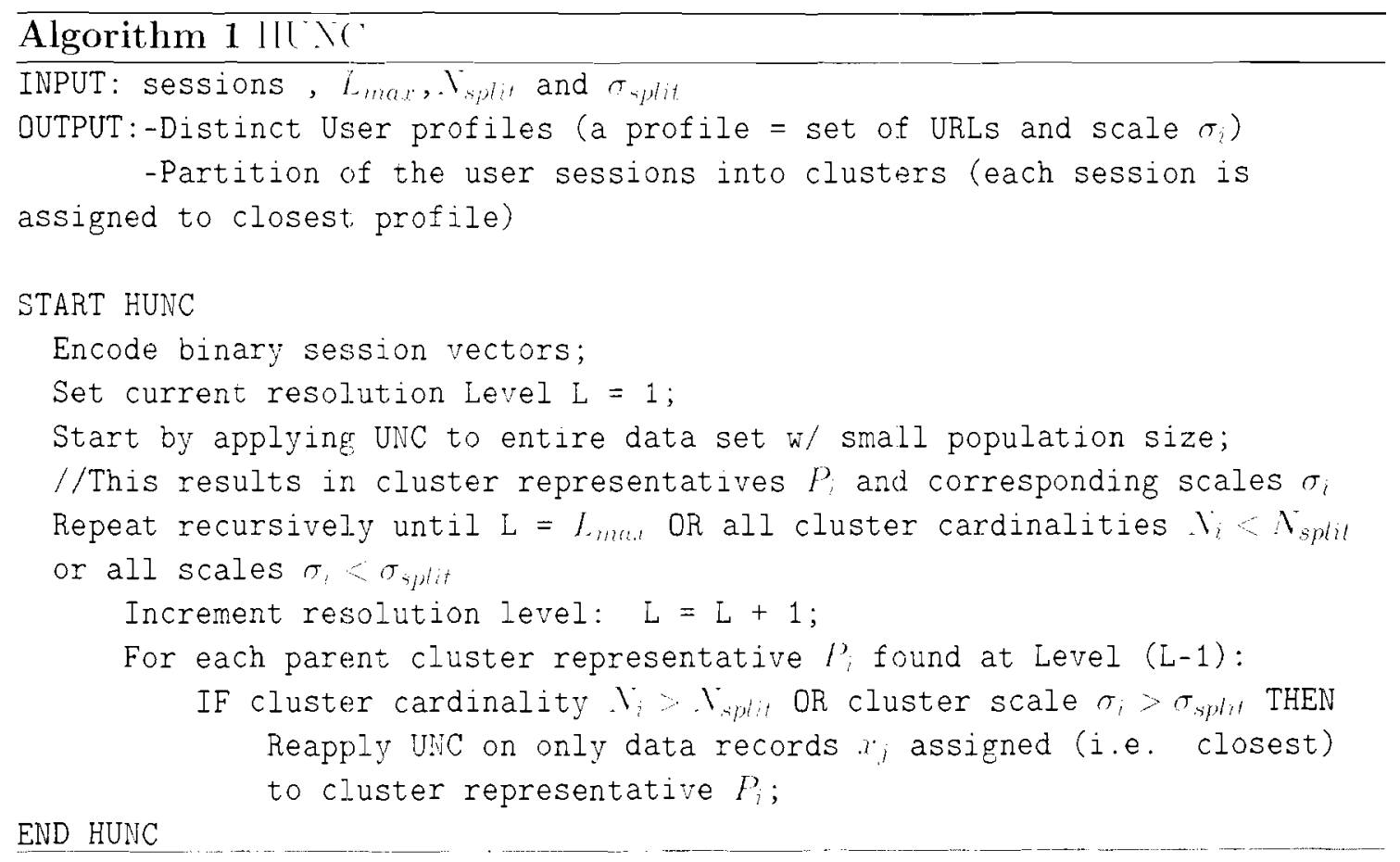

\subsection{Evolving Profiles}

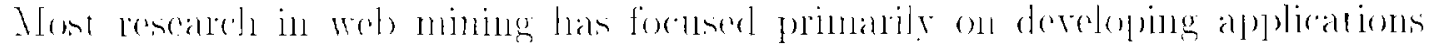

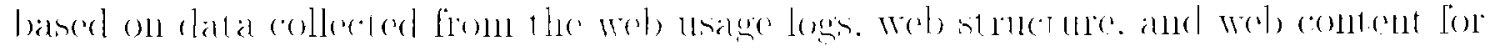

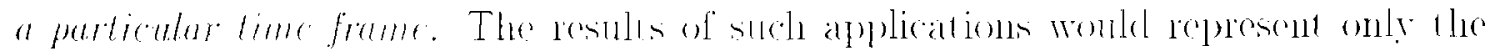

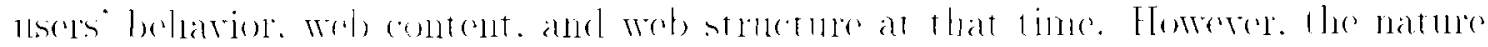

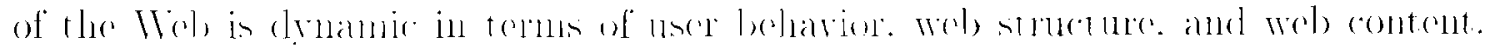

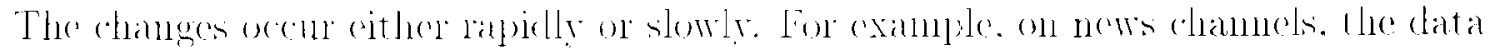

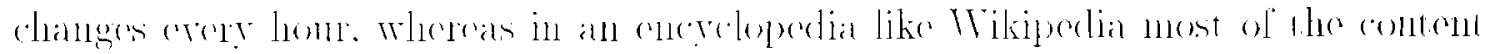

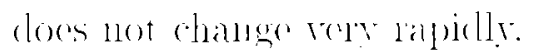

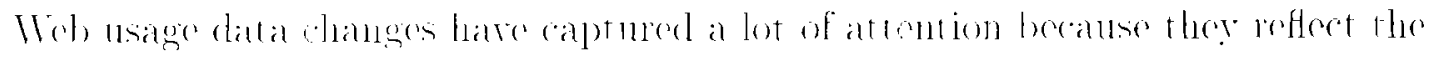

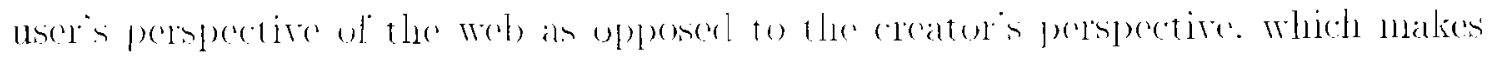

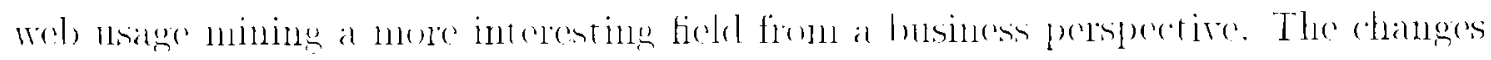

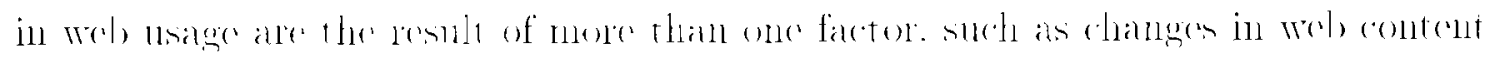

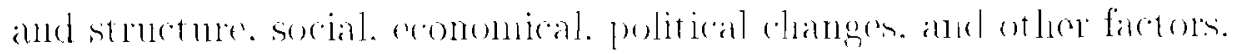

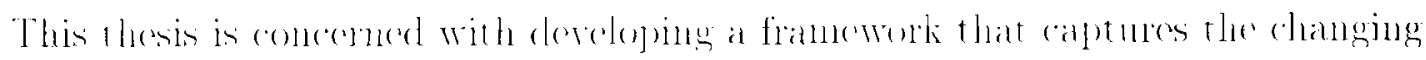




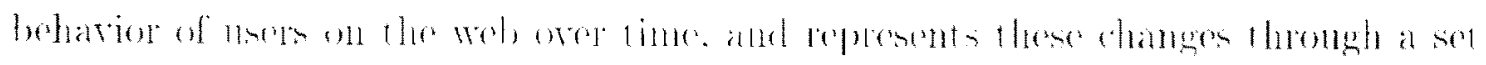

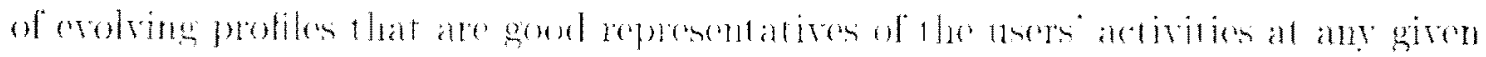
linne.

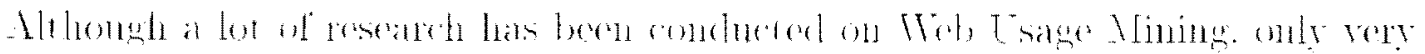

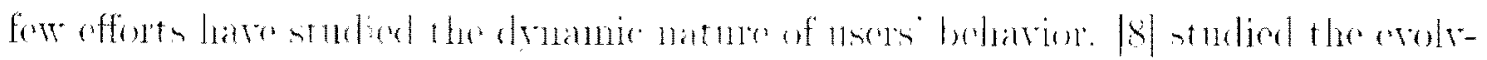

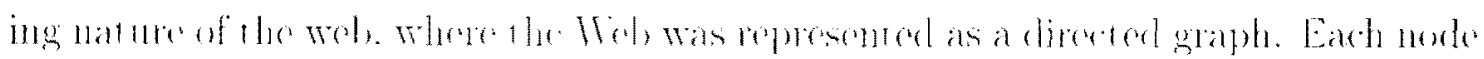

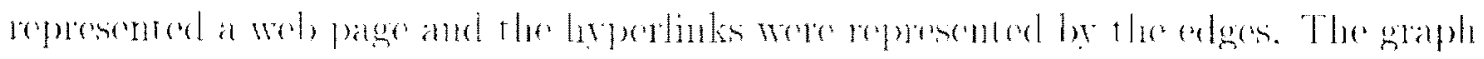

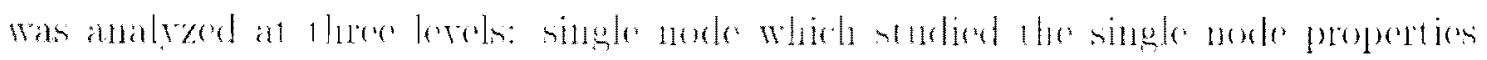

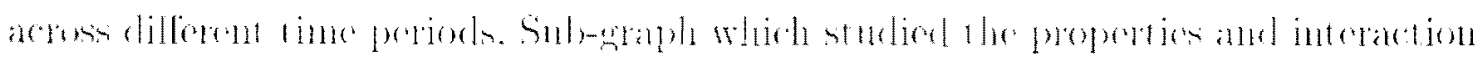

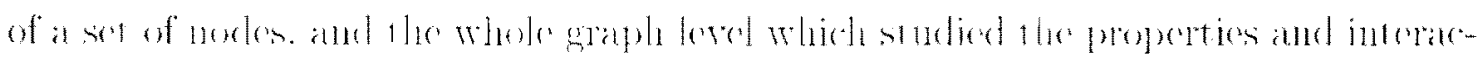

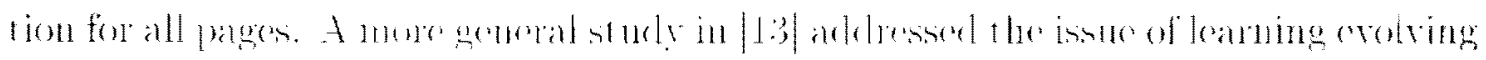

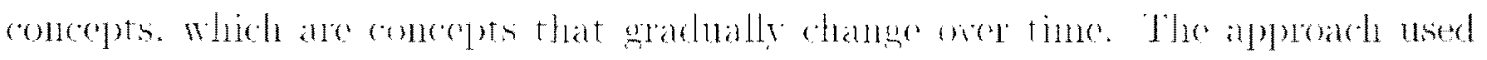

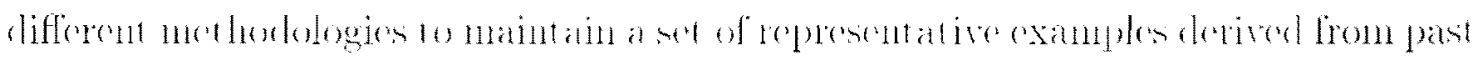

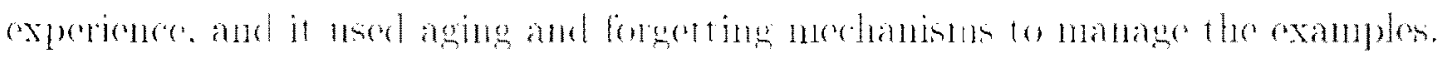

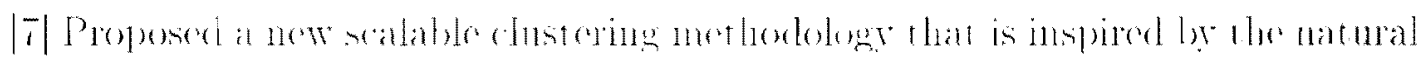

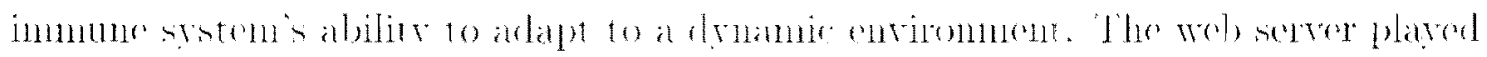

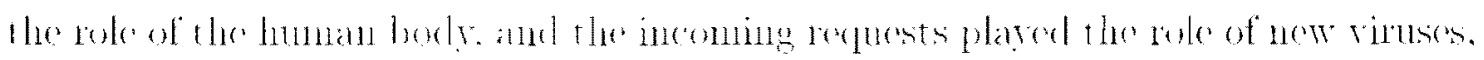

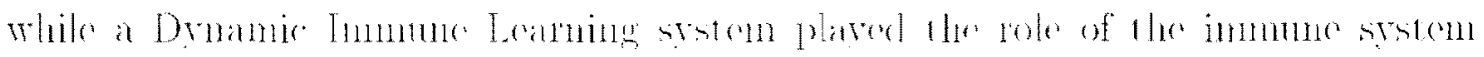
that continnonsty performed an indelligent oreanization of the incoming nerisy data

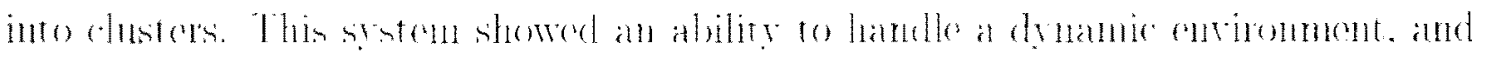

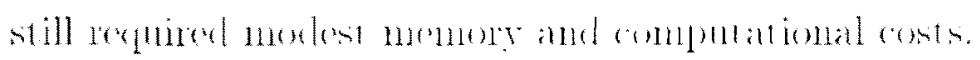

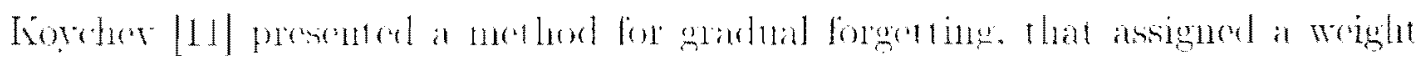

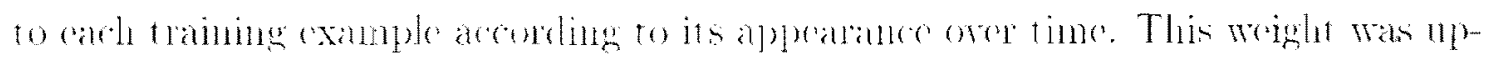

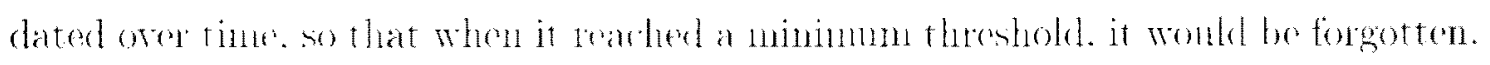
Forcher applied this mothod on the drifting of usens interests. A foature selection

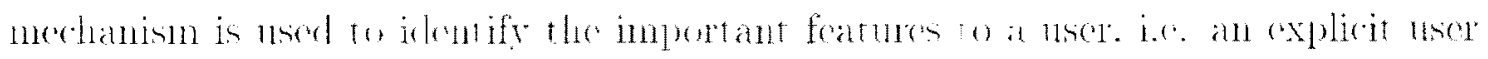

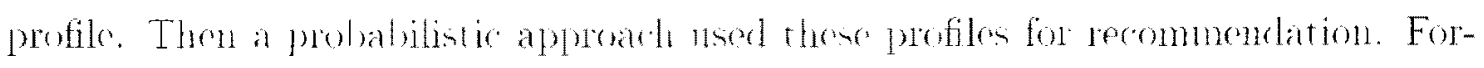




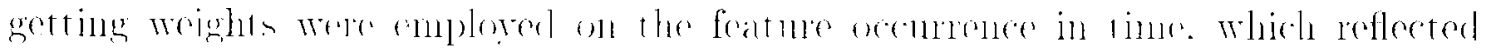

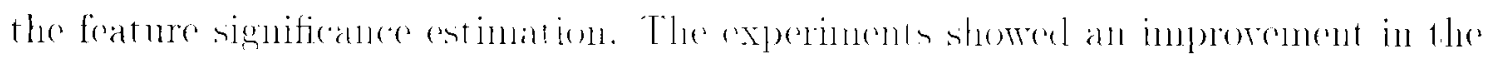

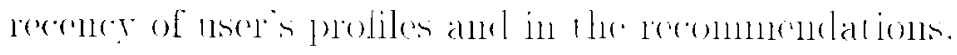

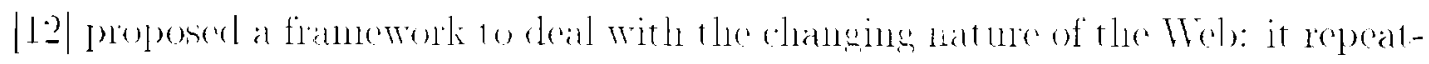

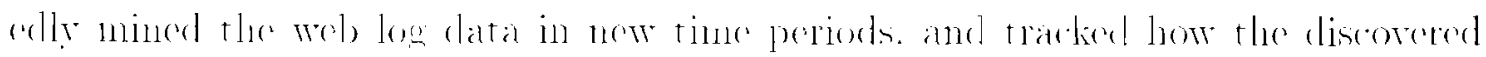

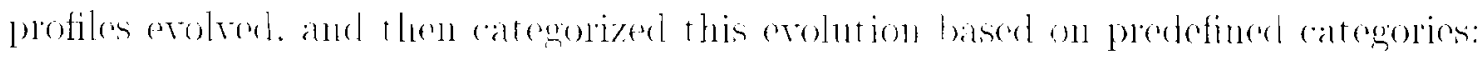

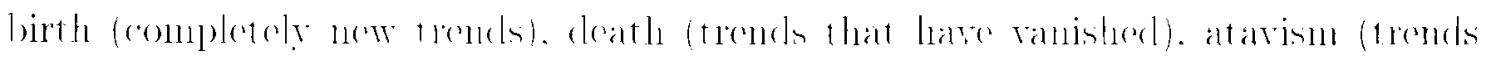

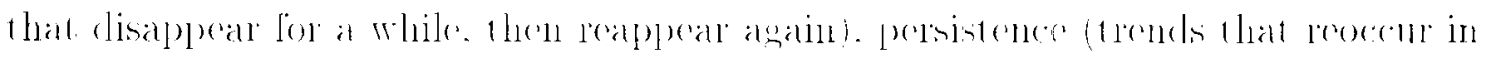

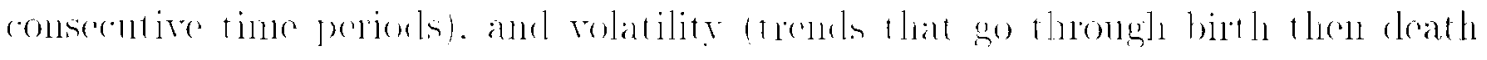

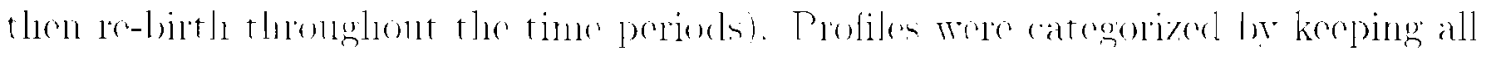

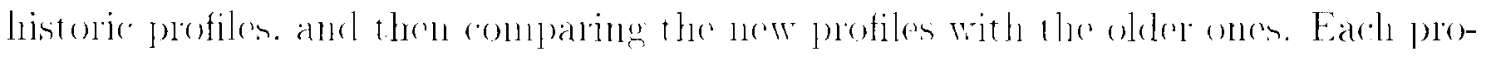

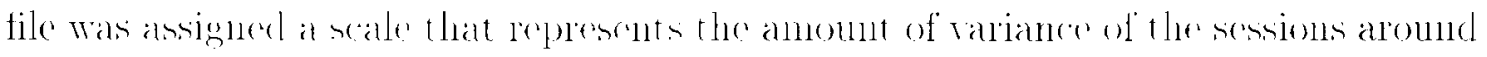

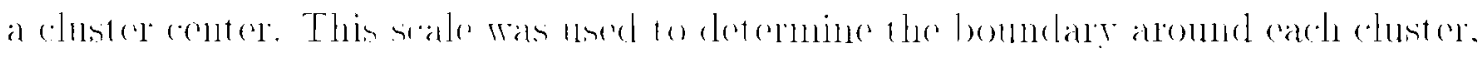

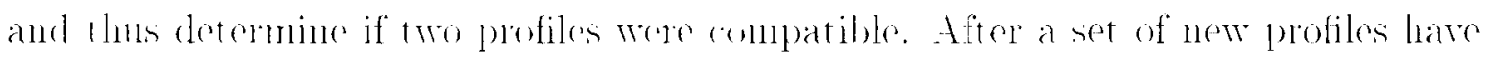

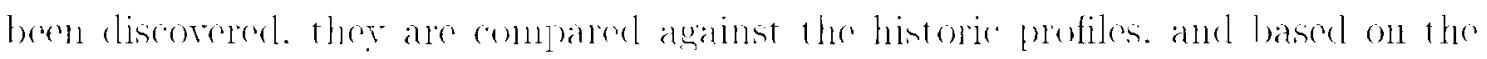

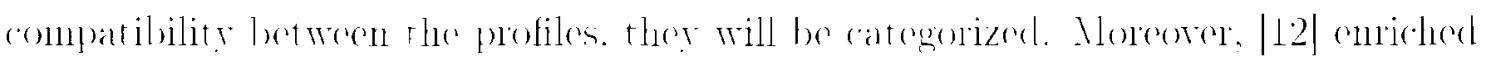

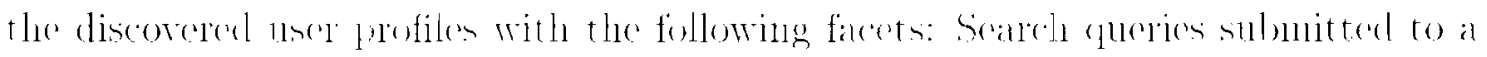

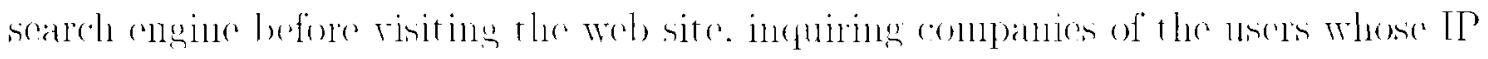

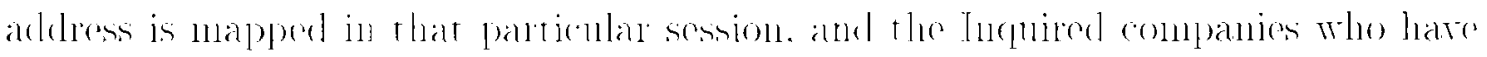

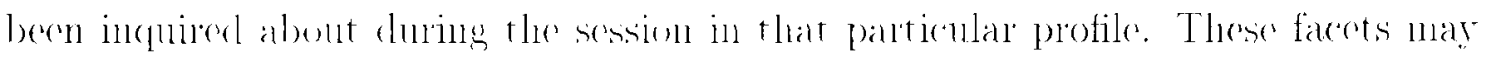

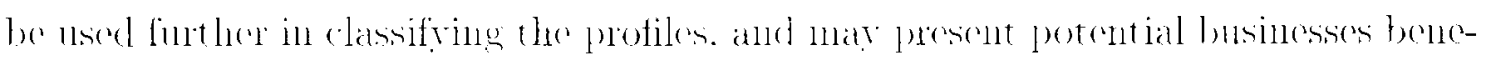

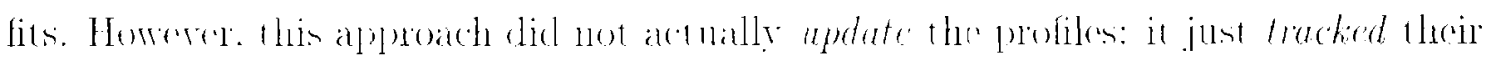

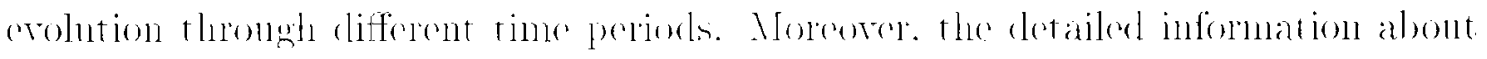
the profilo guality was not maintained.

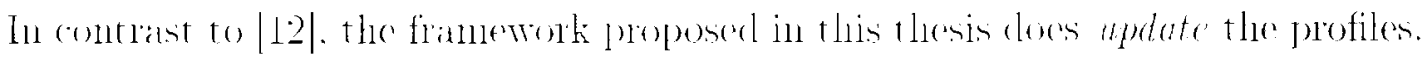

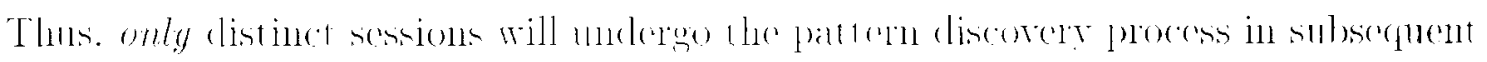

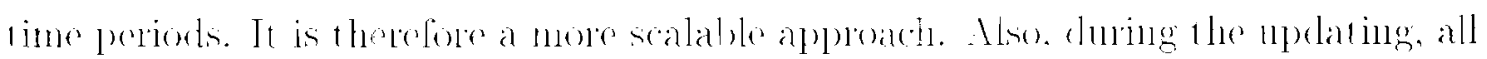




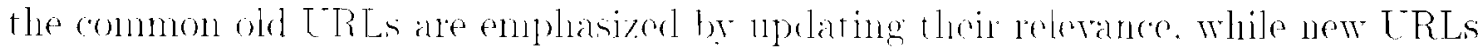

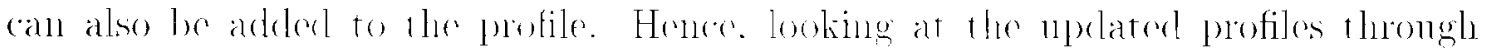

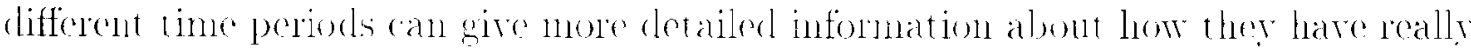
arolverl.

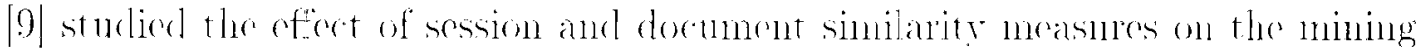
process and on the interperation of the mined patteres in the harsh restrictions

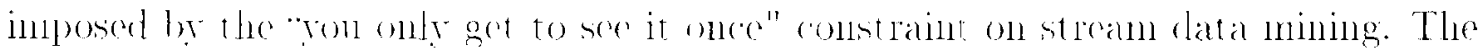

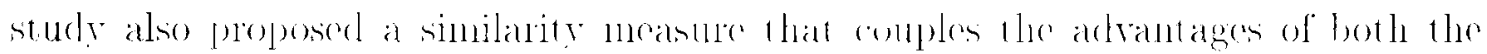

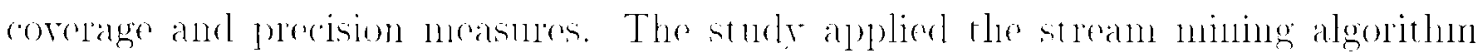

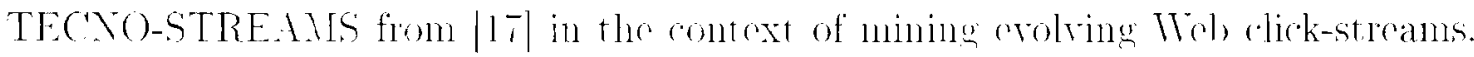

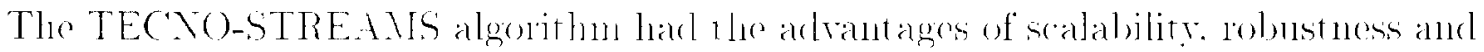
antomatic scale estimation. Vow data is gomeraterl as a stream. and it in processed

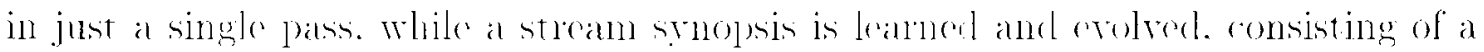

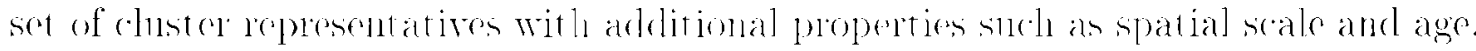

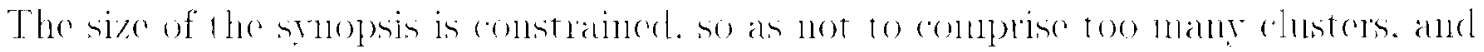

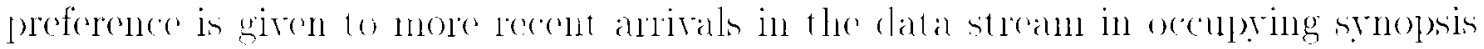
nodes. [9] also presented an inmoratire strategre to ovaluate the discorered trends using somo spocial motries. and a visualizat ion mothos that showod the hits for high precision and high covelage ore tink.

\subsection{Scalability}

Scalability is concertecel with the ability of a srstem or a processis to hamedle a growing andonnt of wort orel time. In the context of wols usage mining, scalability

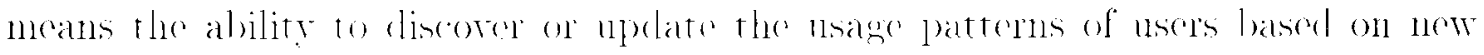

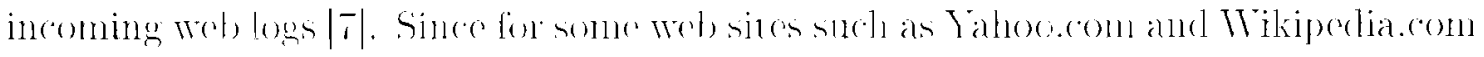

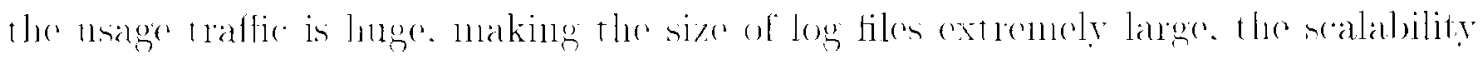

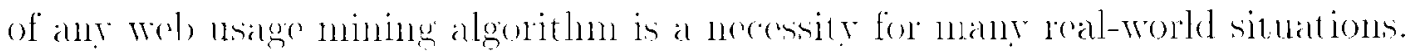




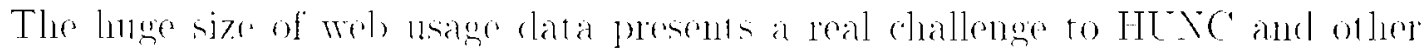

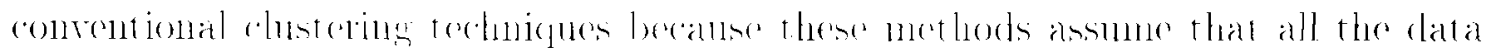

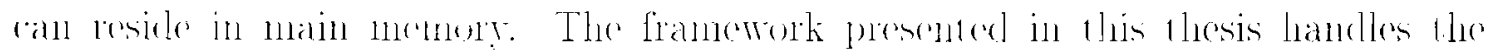

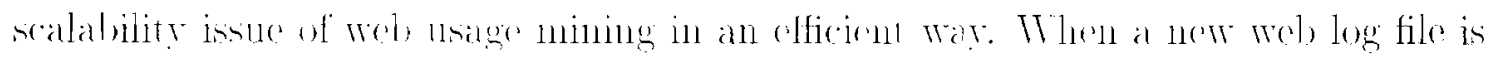

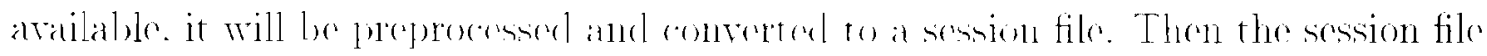

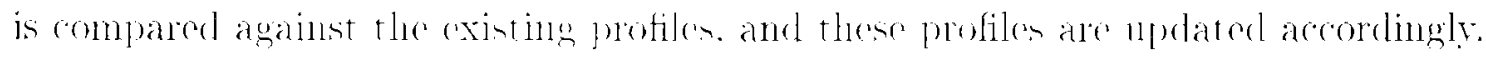

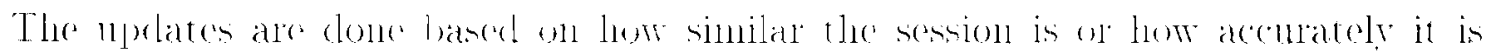
reperesented log some current profile. After the profiles are npelaterl. enly distinct sessions. i.e. sessions that aro not roso enough to any of llo existing profiles. will

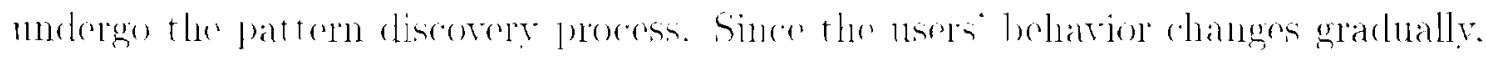

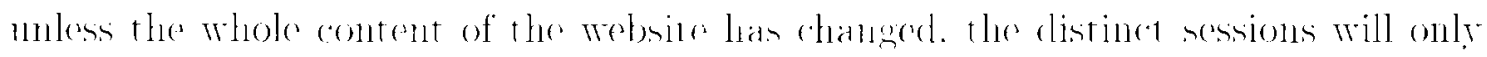

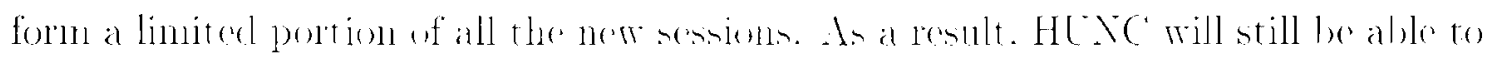

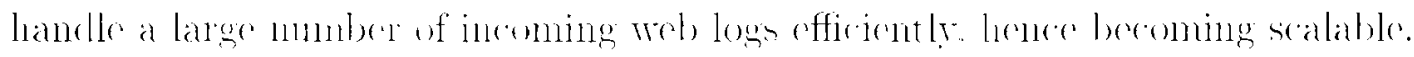

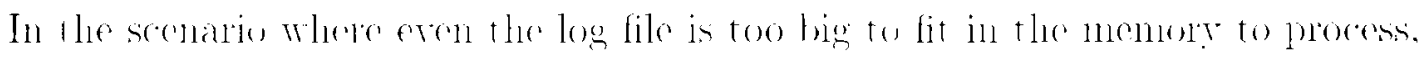
the proposed framework can split the file into a number of hatelos based on pre-

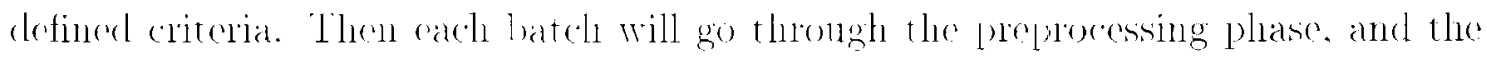

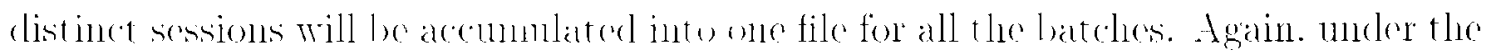

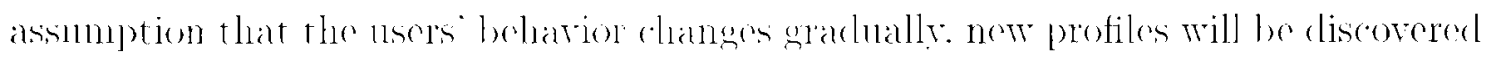
only from llese distinet sesionis.

\subsection{Summary}

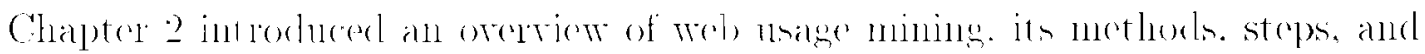
applications. Also, the sounces of unage dara and wolne of the issues related to obtain-

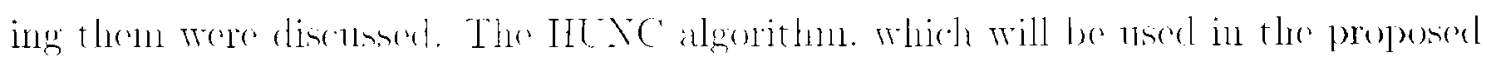

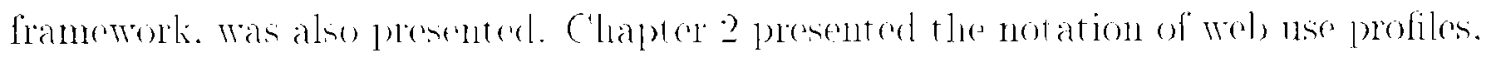

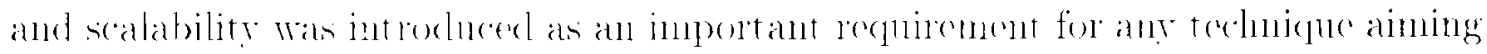

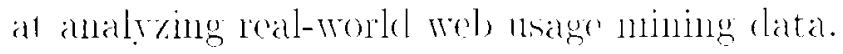




\section{C'HAPTER 3}

\section{METHODOLOGY}

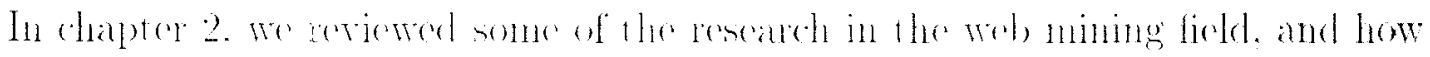

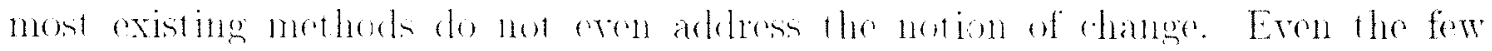
approades that tried to capture and handle the dhanging nature of the web. were

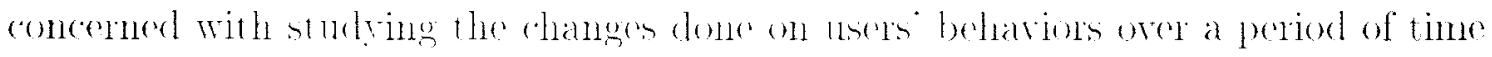
|12|. but failed to utilize this changer divectly to maintain and develop the erolving profiles.

On the other hand. the approach proposed in this thesis discorers the usage patterns orer different periods of tinne. and captures the changes made to the patterns. The olel profiles entemts and properties are upedated as new data arrives, and only

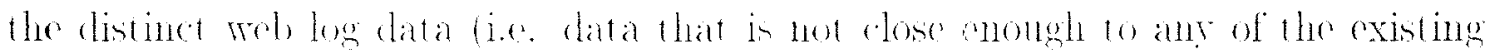
profikes) will medergo the datia mining process. which makes this approach scalable and more efficinent in terms of eomplexity and resomere natage.

When updating a profile. the interesting LTL

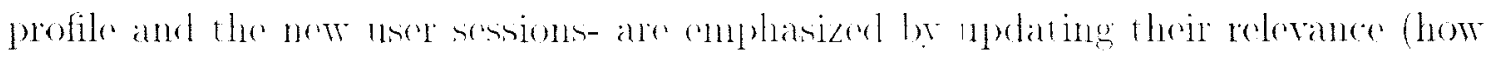

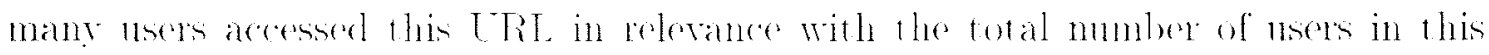
profile). Also the brand ners I RLs in the session are anded to the profile (with the

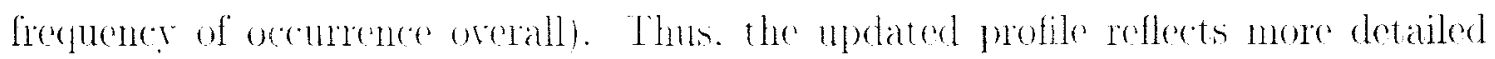
information alout how the nese' beharion changed orer tine. 


\subsection{Overview of the Proposed Methodology}

The weth usage mining procese in traditionally done in sereral steps with only fow variations. The steps an be summarized belew:

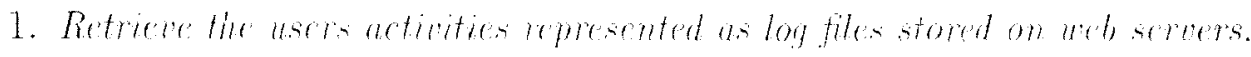

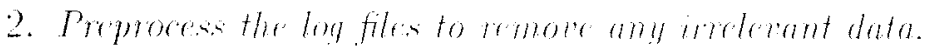

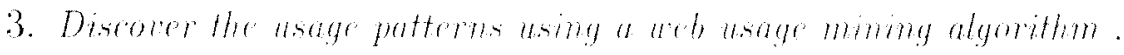

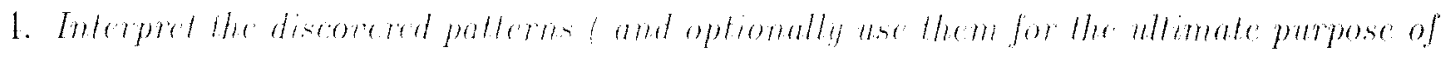
mining. like a recemmendation system).

The traditional main steps abore hate been used to discorel usage pattens withen one specific period of time. but they can argmaldy be reapplied periodically on the web data to tre 10 capture the changes in navigation patterns. Iforever, there are sonur concerns using this approach.

- Reapplying the stops periodically can wither be performed on the whole historie data incheding the newly coming logs. or only on the new log files. The former

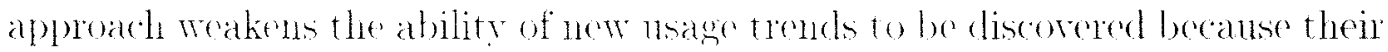

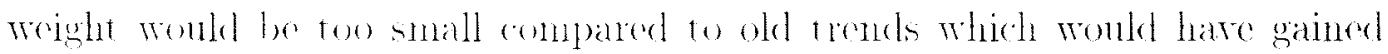
strength orer time. The later completely forgets all previous paterns which may not be reasomable or efficient.

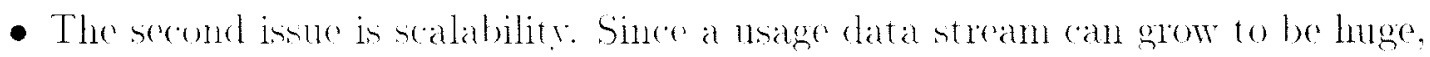
traing to discorer the new behation from the accommlated log filos each time will resuire significant computational resondes and could exen be impractical or impossible for wobsites with huge traffic. 
- The changes in the usage behaviors are not captured in detail, i.e. we do not know how users changed in their behavior. Even though the evolution of an entire profile might be monitored as in [12] and classified, knowing which URLs have changed or became more interesting was not enabled.

To overcome the abore issues, this thesis adds two additional steps. The modified pattern discovery process is shown in Figure 3.1, and can be summarized, assuming that we start with a set of initial (secd) profiles mined from initial period, as follows:

1. Preprocess the new web log data to extract the current user sessions.

2. Update the previous profiles based on the similarities with the extracted user sessions,

3. Re-apply clustering to the distinct user sessions (i.e. the ones not used in step 2 to update the previous profiles) using the Hierarchical Unsupervised Niche Clustering (HUNC) algorithm.

4. Post-process the distinct (new) profiles mined in step 3 ,

5. Combine the updated profiles with the distinct profiles to create the new seed profiles for future mining,

6. Interpret and evaluate the discovered profiles (and optionally use them for the main purpose of mining. like in a recommendation system).

7. Go back to step 1

Figure 3.1 shows the pattern discovery process in three different time periods: the initial time, T1. and T2. The initial period is the first time the web logs are mined, 
Figure 3.1: Proposed Pattern Discovery Process Flowchart

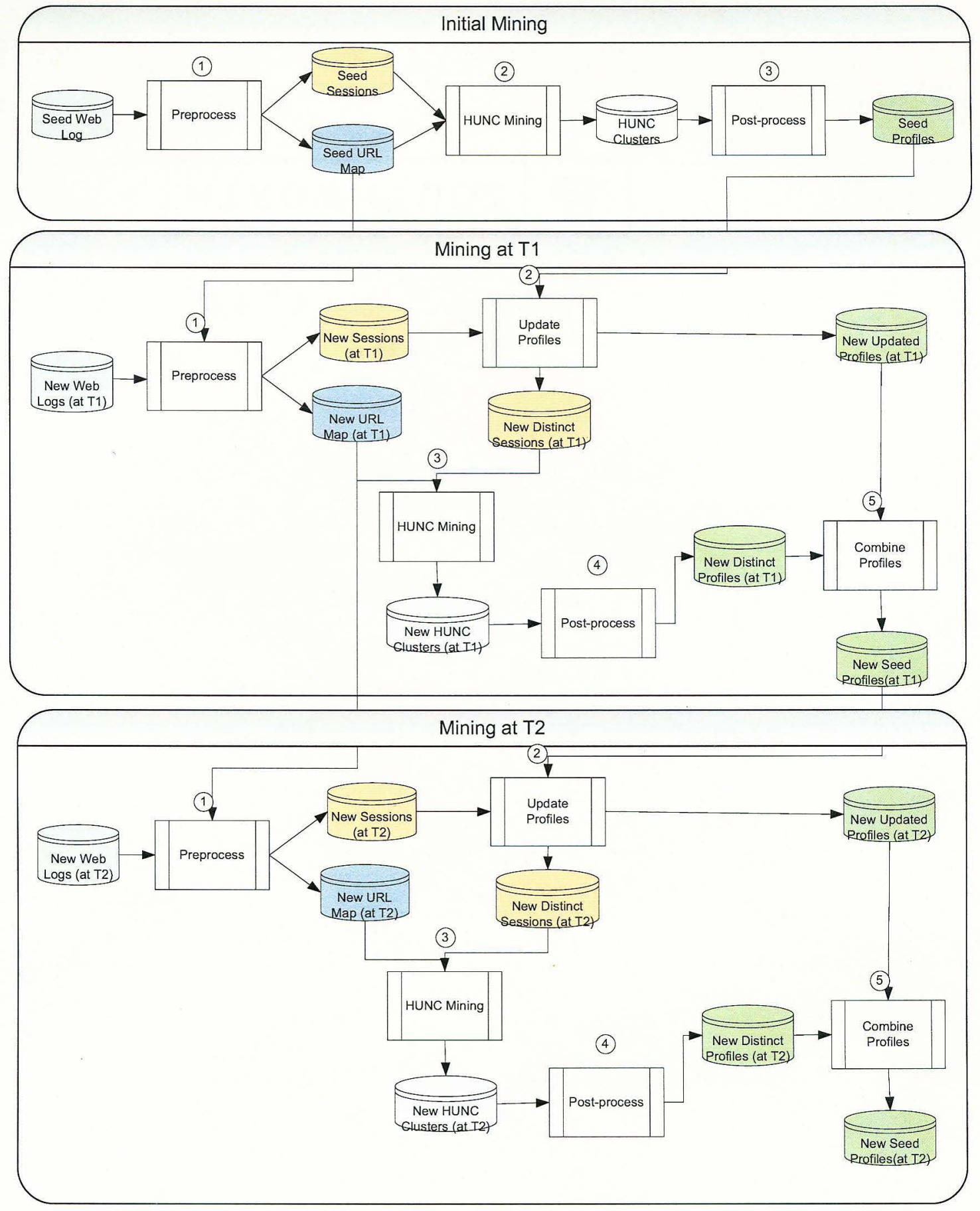


and since there are no historic profiles, all the sessions are considered distinct and they are all used to generate the first seed of profiles.

After the initial period, all the steps above are executed on subsequent time periods $T_{1}, T_{2}, T_{3}$..etc. At each time period $T_{i}$, the profile seed from the previous period $T_{i-1}$ is used in the updating process. Then the distinct profiles are created using the distinct data from $T_{i}$ and combined with the updated profiles to create the profile seeds which will be used as the seed for time period $T_{i+1}$, and so on.

The URL file from $T_{i-1}$ is used in $T_{i}$, which means that the list of all URLs is augmented each time that new LRLs appear in the new data. The reason for this is to keep consistent indexing of all the URLs in the website throughout all the time periods.

\subsection{Preprocessing the logs}

During pre-processing, the web log files are cleaned by removing all irrelevant elements such as images, requests from search agents, and unsuccessful requests. Removing irrelevant accesses is necessary because these elements will affect the accuracy of the discovered patterns, and they will add an overhead to the computational and memory requirements.

Access requests for images are considered irrelevant because the images are typically embedded in a web page, and every time that the web page is requested, these images are requested automatically. Hence, these images do not represent the user's browsing behavior or interests.

Search engines send their web crawlers periodically all over the web to obtain information about web sites, and to index this information to be used in searching. Hence, a web log file will contain many requests from these web crawlers, and these 
entries should be removed since they do not represent an actual Human browsing behavior. A heuristic for removing these requests is by looking for a set of arbitrary keywords like in the "Agent" field, such as "bot" to recognize these requests, or by observing the time difference between subsequent requests. A small time period like 1 second means that the user did not even view the page, so it is most likely a crawler.

After removing irrelevant entries, the page requests are grouped into units called sessions. Each session represents all the pages visited by a particular user within a predefined period of time. The sessions are represented as binary vector as shown in section 2.4.1.

Moreover, a URL index is created that includes all the URLs accessed in the web logs. This index is kept through future pattern discoveries and is always updated to reflect new URLs in the web site. Finally, a matrix of all URL-to-URL similarities, based on Eq. (2.2), is kept. This matrix is used when clustering the sessions.

\subsection{Updating profiles}

During this step, the new usage sessions will be used to update the old profiles, and only the distinct sessions will undergo the next step of discovering the new profiles/trends. The old profiles contribute to the evolving profiles from the last pattern discovery runs. The distinct sessions are the sessions extracted during preprocessing, and which were not used in updating old profiles because they were not found to be "similar" enough to any profile.

Before explaining how profiles are updated, we describe some important properties that describe profiles and sessions. A profile $P_{i}$ is a vector representation of the cluster $X_{i}$, which is a set of user sessions that are more similar to each other than to the sessions in other clusters. The profile is represented as $P_{i}=\left(P_{i 1}, . ., P_{i N_{u}}\right)^{t}$ where $P_{i j}$ is the relevance weight of $U R L_{j}$ in cluster $\mathrm{i}$, and is estimated by the conditional 
probability of accessing $U R L_{j}$ during the sessions assigned to the cluster $X_{i}$

$$
\begin{gathered}
P_{i j}=p\left(s_{j}^{(k)}=1 \mid s_{j}^{(k)} \in X_{i}\right)=\frac{\left|X_{i j}\right|}{\left|X_{i}\right|} \\
X_{i j}=\left\{s^{(k)} \in X_{i} \mid s_{j}^{(k)}>0\right\}
\end{gathered}
$$

$\left|X_{i j}\right|$ is denoted as $N_{i j}$ in the following discussion. The relevance weight determines how much a URL is significant to a profile.

A profile $P_{i}$ has a cardinality $N_{i}$ which is the number of sessions that are closest to the cluster $X_{i}$, i.e. $N_{i}=\left|X_{i}\right|$. The cardinality does not necessary reflect the number of users represented by the profile $P_{i}$, since a user can have more than one session assigned to the same profile, which means that profile $P_{i}$ represents the behavior of that specific user. The equation above can be re-written as:

$$
P_{i j}=\frac{N_{i j}}{N_{i}}
$$

Profile $P_{i}$ has a scale measure $\sigma_{i}^{2}$ that determines how much the sessions in cluster $X_{i}$ are dispersed around the cluster representative. The scale measure can be found using

$$
\sigma_{i}^{2}=\frac{\sum_{j=1}^{N} u_{i j} d_{i j}^{2}}{\sum_{j=1}^{N} u_{i j}}
$$

Where $w_{i j}$ is a robust weight that measures how typical a session $x_{j}$ is in the cluster $X_{i}$.

$$
w_{i j}=e^{-\frac{d_{i j}^{2}}{2 \sigma_{i}^{2}}}
$$

where $d_{i j}^{2}$ is the distance between session $x_{j}$ and the cluster center for $X_{i}$ as given by Eq. $(3.7)$.

The scale measure $\sigma_{i}^{2}$ can be seen as a radius of the profile, and the more similar 
the input sessions are to the profile, the smaller $\sigma_{i}^{2}$. Moreover, based on the robust weight $w_{i j}$ it is possible to detect outliers which will have small weights, thus offering a means of distinguishing between good data and noise.

The similarity between a session $\mathrm{S}$ and a profile $\mathrm{P}$ can be assessed using the Cosine Similarity between two sets as follows:

$$
\operatorname{Sim}_{c o s}(P, S)=\frac{|P \cap S|}{\sqrt{|P| \cdot|S|}}
$$

Where $|\mathrm{P}|$ is the number of URLs in profile $\mathrm{P}$, and $|\mathrm{S}|$ is the number of URLs in session $\mathrm{S}$. The cosine similarity increases as the profile and the session share more common URLs, and it is normalized to be in the range [0-1].

The similarity between sessions $\mathrm{S}$ and profile $\mathrm{P}$ is mapped to the dissimilarity or distance:

$$
d_{S, P}^{2}=\left(1-\operatorname{Sim}_{\cos }(P, S)\right)^{2}
$$

Another measure of similarity called Robust Weight Similarity, will be used in the experiments, where we use the weight of a session defined in Eq.(3.5) with respect to the profile and compare it against a threshold value. The advantage of using the robust weight would be that the weights are normalized by the scale of each cluster; hence, they are less sensitive to the threshold, and they also depend on the profile. Profiles with smaller scales will be more strict in matching and vice vers for large-scale profiles.

One important issue when finding the similarity between a profile and a session is to consider only significant common URLs. The URLs in the profile are thus first compared to a threshold value $P_{\min }$, and only URLs that pass this threshold values will be considered toward the count of similar URLs that will be used in finding the Cosine Similarity or the Robust Weight Similarity. Thresholding URLs is necessary to filter out the effect of potential outliers that might divert the profile from its accurate 
and natural development. However, a too strict thresholding might cause discarding important updates to profiles in their infancy. Hence, choosing the right threshold value is vital. Updating the profiles can be summarized in algorithm 2 .

Update_Profiles compares each session with all the old profiles, using the similarity between the session and each profile. The closest profile is chosen and its similarity is compared to a threshold value $\operatorname{Sim}_{\min }$ and, if it exceeds this threshold value, then it will be updated. Otherwise the session will be classified as distinct.

Only the closest profile is updated in this approach, even though more than one profile might be close to the session. This approach is called the hard or crisp updating, where the session is a member of only one profile. An alternative approach based on soft or fuzzy memberships could allow a session to be a member of more than one profile. In this case, all profiles that are close to the session should be updated. There is a trade-off depending on which membership to use. A soft membership is expected to cause the coverage to increase, since the session will be represented in more than one profile. However, a higher coverage will almost always cause a lower precision, since the profiles would become more general and thus less detailed or accurate. A hard membership represents higher precision and lower coverage. From a business perspective, a hard membership means that each user is mapped to exactly one profile, making more detailed information about his behavior available, whereas, a soft membership will map each user to more than one profile, making more cross-product recommendations possible, at the risk of losing detailed information about the user's behavior.

In the UpdateProfile procedure, first the cardinality of the profile is incremented by one because the profile is accepting an additional session. Second, the URLs in the session are compared to URLs in the profile, and if the URL from the session is in the profile, then its weight is incremented because this URL is now found in an additional session. If the URL from the session does not exist yet in the profile, then 

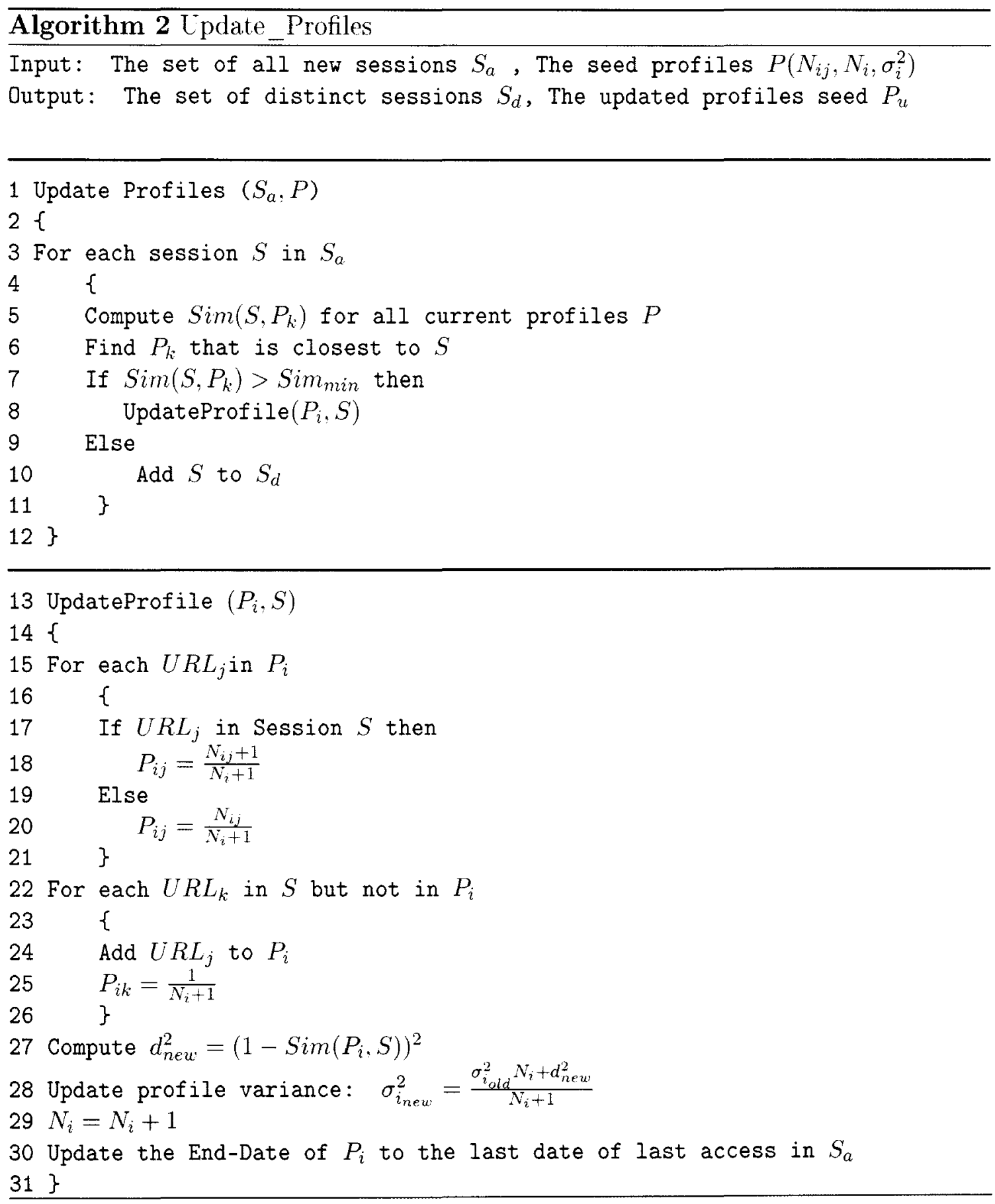
it should be added, and its weight will be initialized. This weight will be low at the beginning because this URL is only in one session, however it should increase with time if this URL is interesting. All the URLs already in the profile, but that do not exist in the session will maintain their weight, but this weight will decrease slightly since the profile cardinality has increased.

Each profile contains a starting date and ending date, which reflect the period of time that this profile covers. This is important to keep track of which profile is most recent and which one was not recently updated. During the updating process, only the ending date is updated to reflect the date of the last access in the web log being processed. These dates could be used further during maintenance to identify which profiles become obsolete and should be archived.

Finally, the variance of the profile should be updated. As shown in line 28 in algorithm 2, the variance is an incremental version of the ratio of sessions' distance to the profile divided by the profile cardinality. Since the profile acquired a new session now, the new session's distance $\left(d_{n e w}^{2}\right)$ should be added to the variance. The weight of each new session is considered to be 1 (unlike the variance definition in Eq.(3.4)), since we are already restricting only very similar sessions to update the profile (thus outliers are eliminated).

The complexity of Algorithm 2 is

$$
O\left(N_{s} *|P| * \operatorname{Max}\left(|U R L|_{P},|U R L|_{S}\right)\right)
$$

where $N_{s}$ is the number of new sessions, $|P|$ is the number of profiles, $|U R L|_{P}$ is the maximum number of LRLs in a profile, and $|U R L|_{S}$ is the maximum number of URLs per sessions.

Furthermore, we exploit the fact that Web sessions are extremely sparse (typically 10 URLs per session) as well as profiles, especially when applying a threshold on 
URL significance. Hence, the maximum number of URLs is typically $<10$. Moreover, the number of profiles tends to be small because only strong profiles are mined at any period. In the cleaning process discussed later, obsolete profiles can be deleted as well. Thus, the number of sessions is what really affects the performance of the updating algorithm. However, an important thing to remember is that updating of the profiles will most likely be done offline, so that it does not add any overhead in real-time.

\subsection{Discovering distinct profiles}

After updating old profiles, the new user sessions are analyzed so that new usage patterns can be discovered. These new trends may represent a potential new interesting market niche, or a radical change in the current market needs and behavior.

After the old profiles have been updated, only distinct sessions will undergo the pattern discovery process. The HUNC algorithm described in Section 2.4, is used to discover the new patterns, and the output will be denoted as the new profiles.

The output of the pattern discovery process at period $(\mathrm{t})$ is a set of new clusters or profiles that are represented as a set of URLs. Each cluster has a variance measure $\sigma_{i}^{2}$, cardinality measure $N_{i}$, sum of weights, and a density or fitness. The variance $\sigma_{i}^{2}$ is defined in Eq.(3.4), the cardinality is the number of sessions assigned/closest to this cluster up to period $(\mathrm{t})$ and is donated as $N_{i}$ as described in Section 3.3. The Density $f_{i}$ of Profile $\mathrm{i}$ is defined as:

$$
f_{i}=\frac{\sum_{j=1}^{N_{i}} w_{i j}}{\sigma_{i}^{2}}
$$

where $w_{i j}$ given by Eq.(3.5).

Since the distinct sessions are only part of the original sessions, the HUNC run time and resource usage can be reduced, which increases the scalability of this approach. 
Figure 3.2: Sample Profile

Profile: 9, Num.URLS: 11, Cardinality: 58

StartDate: 30/Jan/1998:17:30:33 , End Date: 04/Feb/1998:13:37:34,

Variance: 0.0857

$\{0.98-/$ courses.html $\}$

$\{0.98-/$ courses 100. html $\}$

$\{0.96-/$ courses_index.html $\}$

$\{0.82-/\}$

$\{0.74-/$ cecs_computer.class $\}$

$\{0.34-/$ courses $300 . \mathrm{html}\}$

$\{0.20-/$ courses $200 . \mathrm{html}\}$

$\{0.17$ - /courses_webpg.html $\}$

$\{0.12-/ \sim$ joshi/courses/cecs352\}

$\{0.10-/$ courses 400. html $\}$

$\{0.10-/$ people.html\}

\subsection{Post-processing the distinct profiles}

The purpose of post-processing is to formulate the discovered patterns in a way that is understandable by humans, and is usable by higher-level applications.

The post-processing phase is the same as in HUNC, and it will be applied only on the newly discovered clusters. During the post-processing phase, each URL is mapped to the closest cluster. The set of all URLs in the same cluster constitutes a profile. A relevance weight is calculated for each URL as in Eq.(3.5), and the profile variance and cardinality are calculated as well - which are the same as in the cluster.

A sample profile is shown in the Figure 2.7. This profile contains 11 URLs, represents 58 sessions, and reflects the web activity between Jan 14th 1998 and Feb 4th 1998 . Note that the profile variance is relatively small $(0.0857)$, which means that the URLs are very close to each other.

\subsection{Combining profiles}

In this phase, the updated profiles and the newly discovered profiles are combined into one set of profiles that will serve as the new seed of profiles for the next pattern 
discovery period.

Also in this phase, statistics about the pattern discovery process are collected, such as the number of updated profiles, the number of newly discovered profiles, and the number of distinct sessions. These statistics help in monitoring the performance of the discovery process, and in analyzing the output. For example, they can answer questions such as:

"During which period did the browsing behavior change the most?"

"Did changing the content of the website in period T1 change the users behavior?"

\subsection{Interpreting the profiles}

The main purpose of web usage mining is to discover interesting information from raw data in web access log files, and to utilize this information in meeting some business or information organization goals. Thus, after summarizing the browsing behavior of users in the form of profiles, these profiles could serve as the input to higher-level tools that can analyze the usage patterns and make conclusions that help in the decision making process. The analysis tools draw on a mix of different fields including statistics, graphics, visualization and database management. Visualization can offer a successful mean to help people better understand and study various kinds of output.

The proposed framework generates well-formulated profiles that contain information and statistics about the profile and its contents. Some of the information is descriptive and can be used directly in higher level application. This includes the start and end date of the profile which mark the time period that this profile represents, as well as the list of LRLs in that profile. Other types of information are mathematical, and are necessary to put the profile in perspective with other profiles, and each URL in perspective with other URLs in the same profile. This information includes the profile variance, profile cardinality, and URL weights. These statistics 
are normally used when trying to decide which profile best represents a specific user, and which URLs are the most significant.

There are also some other pieces of information that are gathered for each pattern discovery cycle, and used for a higher level analysis of the profile evolution and interaction. For each cycle, the following statistics are collected:

1. The number of distinct profiles created in this cycle, which reflects which period witnessed the most and least radical changes in user behaviors.

2. The number of static profiles, which are the profiles that did not get any new updates from the previous cycle. This might signify the presence of out-dated profiles (since no new session matched them).

3. The number of distinct sessions, which could be used in evaluating the scalability performance of the usage discovery process.

\subsection{Profile Maintenance}

During the updating process, a few properties could be changed in the profiles: New URLs might be added, existing LRL weights may change, and profiles variances may get updated. However, over time, some of the profiles or the URLs may become obsolete and should be omitted for several reasons:

- Profiles are too old and they have not been updated for a long time, so they do not represent interesting and recent usage patterns.

- URLs were removed from the web site, so keeping the URLs might have caused directing the users to non-existing pages.

- The URL relevance weight is too low, and it is not improving. This means that 
this URL is not gaining any interest from the users, which could be because it is old, not accessible, or it appeared only once and disappeared.

- The profile represents only a small number of sessions, which is not cost effective from a business intelligence perspective.

- A profile is becoming too general, as indicated by a very high variance. In this case, the profile no longer represents a set of very similar user sessions. Hence it is of poor quality.

The existence of these obsolete profiles and URLs may cause the output of the pattern discovery to be inaccurate and misleading, and may even result in the dissatisfaction of users, in case the profiles are used to compute recommendations (e.g. a user might be directed to a non-existing or unrelated page). Moreover, these obsolete profiles add to the overhead in computations during the updating process.

Profile maintenance should be conducted in order to get rid of all obsolete profiles, and to make sure that the profiles are accurate, compact and up to date. The maintenance could be done by checking the profiles against some threshold values for the profile variance and URL weight. Given a maximum profile threshold $\sigma_{\max }$ and a minimum URL weight $P_{\min }$, Algorithm 3 can be used for maintenance.

The complexity of the maintenance algorithm is $O\left(|P| * \operatorname{Max}\left(|U R L|_{P_{a l l}}\right)\right)$, where $|\mathrm{P}|$ is the total number of profiles, and $|U R L|$ is the number of URLs in a profile. Both these values are relatively low. Besides the profile maintenance algorithm can be executed offline, so that maintenance does not add an overhead to computations.

One important thing to notice about maintaining profiles is that the URL weights are compared against the threshold value before comparing the profile variance against 


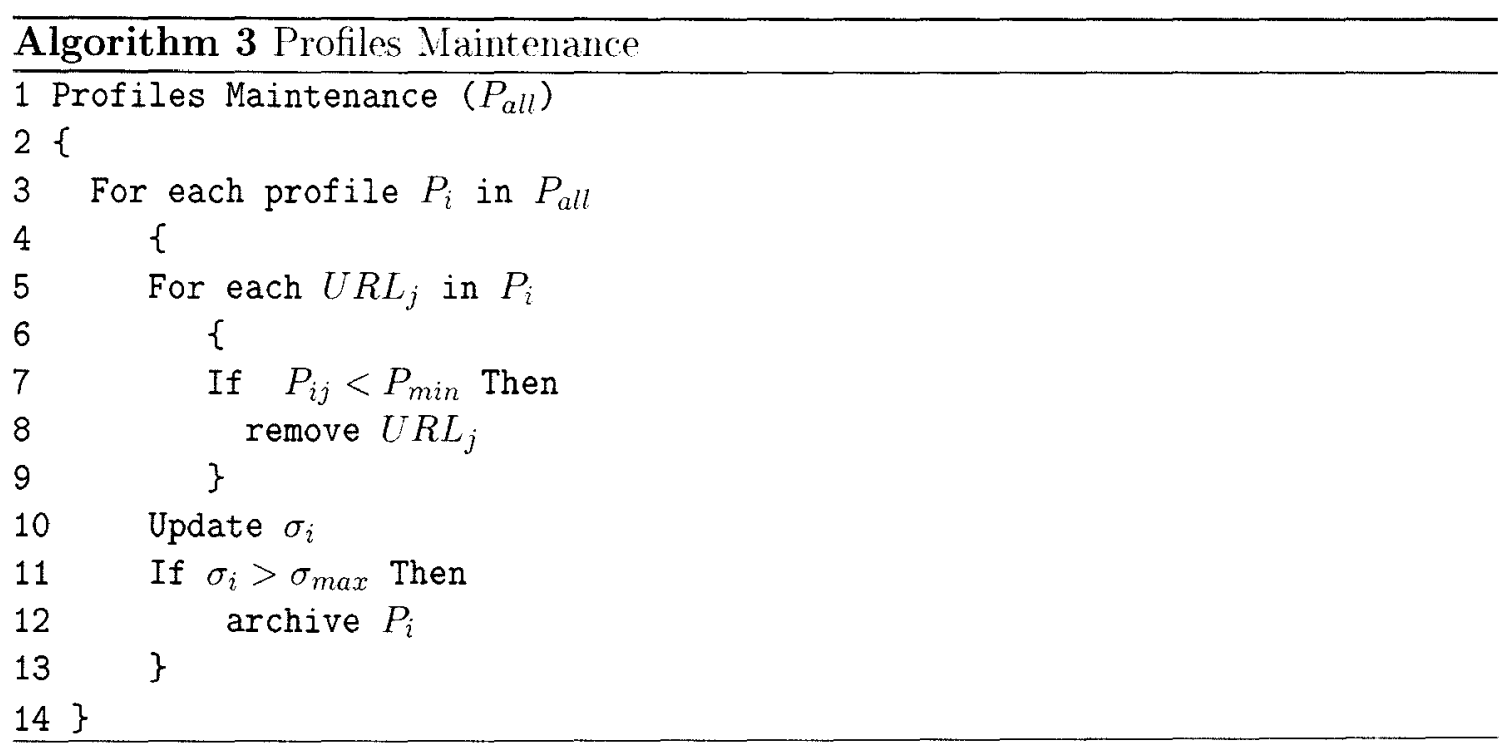

the threshold. This is important since deleting URLs will change the variance. Hence, after these deletions, the profile could become more interesting.

Algorithm 3 is pretty straightforward. However some important issues need to be addressed. The first issue is what threshold value to use; while the second issue is deciding when to run the profile maintenance algorithm.

Choosing the right threshold value is usually done by trial and error, and is domain-dependent. The profile variance represents how much a profile is dispersed, i.e. how different the covered sessions are from this profile. The variance value is in the range [0-1], so a high variance might mean that this profile covers a wider set of sessions, e.g. the profile contains accesses to URLs from different departments in an on-line store. A high variance is not desirable since it might be a result of a diversion of sessions from the original profile seed during updates, or it could signify that this profile represents two clusters, and hence it should be split in 2 profiles. On the other hand, a lower variance means that this profile contains sessions that are very similar to the profile. This is more desirable since it means that the profile is from a very compact cluster/group of users that truly represent a homogeneous interest.

Hence, choosing a threshold depends on the high-level application that will utilize the profiles; a news website might prefer more detailed profiles (so the variance 
threshold would be low). On the other hand, an on-line store might occasionally prefer more general profiles for more cross-products recommendation between different departments (so the profile variance threshold value would be higher).

Another observation is that profiles are archived and not deleted. This means that those profiles that become inactive (no more sessions are similar to them) can still be useful when conducting some analysis on the profiles evolution over a longer time, and could be compared with active profiles to identify any similarities that might indicate a behavior group that was lost for a while and then came back.

The URL weight represents how much that URL is significant in the profile. The weight value is in the range [0-1]; a higher weight means that it is more significant, i.e. more sessions accessed this URL. Again, choosing a right threshold value is a domain-dependent task, so if the recommendations are required to be compact and accurate like in recommending a book, then choosing a high threshold is desirable.

A more complex issue is deciding when to run the maintenance algorithm. During the process of updating profiles in section 3.3, some new URLs will be added to the existing profiles, and their weight will be set to a small value $\frac{1}{N_{i}+1}$. hence, the maintenance algorithm would most likely remove these newly added URLs.

Moreover, in the process of discovering new profiles in Section 3.4 completely new profiles are created, and their variance might not be desirable, which might cause the maintenance algorithm to remove these newly created profiles. However, in both scenarios above, the newly added URLs might gain more weight over time so they would become interesting, and the newly created profiles might change their variance to a desirable value given time. Therefore, the timing of maintenance is crucial since it might cause the loss of an interesting and premature profile or LRL.

As in choosing the threshold values, choosing the right time to run the maintenance algorithm is not trivial. A heuristic rule is to run it periodically with enough time in between, so that the profiles and URLs will have enough time to develop and stabilize. 
Even in the scenario where a URL is created just before the maintenance time, and even if it was deleted, then, if it was really interesting, it will be added again and might gain weight in the next cycle.

Choosing the right time period is domain-dependent. A news website would probably perform the mining on a daily or even hourly basis since new URLs are added every hour. On the other hand, an on-line store would probably run its maintenance every month, with new events (e.g. introducing new products), or when marketing campaigns are started.

\subsection{Summary}

The proposed methodology, for mining and tracking evolving user profiles, was presented in Chapter 3. The main steps of tracking evolving users' behavior were discussed. These are: preprocessing new usage data, updating current profiles based on the new usage data, discovering usage profiles only from distinct usage data, post-processing the newly discovered profiles, combining updated and discovered profiles, and interpreting and evaluating the final profiles output. Moreover, Chapter 3, proposed a maintenance algorithm that is necessary to keep the discovered profiles accurate, compact, and up to date. 


\section{CHAPTER 4}

\section{EXPERIMENTS}

To evaluate the performance of the proposed framework, it was used to discover the usage patterns from real web sites. Two web sites were used: University of Missouri's CECS department's web site, and University of Louisville's library website (library.louisville.edu). For each web site, a set of evolving usage profiles were extracted, and their quality was evaluated using a set of metrics. This chapter starts by defining these evaluation metrics in Section 4.1, then discusses the different parameters that will be used and their configurations in Section 4.2. Section 4.3 will discuss the experimental configurations. The results for each web site are presented in Section 4.4 and Section 4.5.

\subsection{Evaluation Metrics}

The output of the knowledge discovery process is a set of profiles, each of which is a set of URLs with additional metrics. Some of these profiles can be evaluated manually, by visiting its URLs and trying to determine whether these links are related or might represent a plausible usage trend. However, this evaluation can be subjective since what makes one user go from one page to another might be different from other users. Besides, it can be hard or even outdated to visit each URL for large profiles especially once URLs change location and content. 
Table 4.1: Evaluation Metrics Summary ( $\mathrm{t}=$ time period index)

\begin{tabular}{|c|c|c|c|}
\hline Evaluation Metric & Notation & Desired Value & Range \\
\hline \hline Profile Variance & $\sigma_{i}^{2}$ & Low & {$[0-1]$} \\
\hline Profile Cardinality & $N_{i}^{t}$ & High & {$\left[1-N_{s}\right]$} \\
\hline Matching Sessions & - & High & {$\left[1-N_{s}\right]$} \\
\hline Profiles Count & - & High & {$\left[1-N_{s}\right]$} \\
\hline Profiles Pair-wise Similarity & $\operatorname{Sim}_{\cos }\left(P_{i}, P_{j}\right)$ & Low & {$[0-1]$} \\
\hline Profile Density & $D_{i}^{t}$ & High & {$[1-\infty]$} \\
\hline
\end{tabular}

Also, it is not enough that the profile "descriptions" are plausible, since an additional criterion is that the profiles form good "clusters". A good clustering result is one, where data in the same cluster are very similar, while being dissimilar from data in other clusters. Another way to express this is that the clusters (or profiles) should be compact and separated. Thus, a more reliable and accurate method is to use a set of objective evaluation metrics to assess the quality of profiles. Table 4.1 lists the evaluation metrics and their expected ranges to be described in the next sections in detail ( $N_{s}$ is the number of sessions in the data).

\subsubsection{Profile Variance}

The profile or cluster variance (sigma) was defined in Eq.(3.4), and reflects how much the sessions in that cluster are dispersed. The profile variance is normalized in the range [0-1], and its value approaches 1 as the sessions in the corresponding cluster get further from each other ( i.e. are less similar to each other). Hence, a lower profile variance is desirable, because it means that the sessions are closer to each other, i.e. the usage patterns for these sessions are similar, and this in turns attests to the quality of this profile.

The profile variance is proportionally related to the dissimilarity (difference) between the session and a profile. As the dissimilarity increases (i.e. sessions gets further from the cluster's core), the variance will increase. The dissimilarity was defined in Eq.(3.7). The cosine similarity can be redefined using the dissimilarity as: 
Figure 4.1: Cosine Similarity vs. Profile Variance

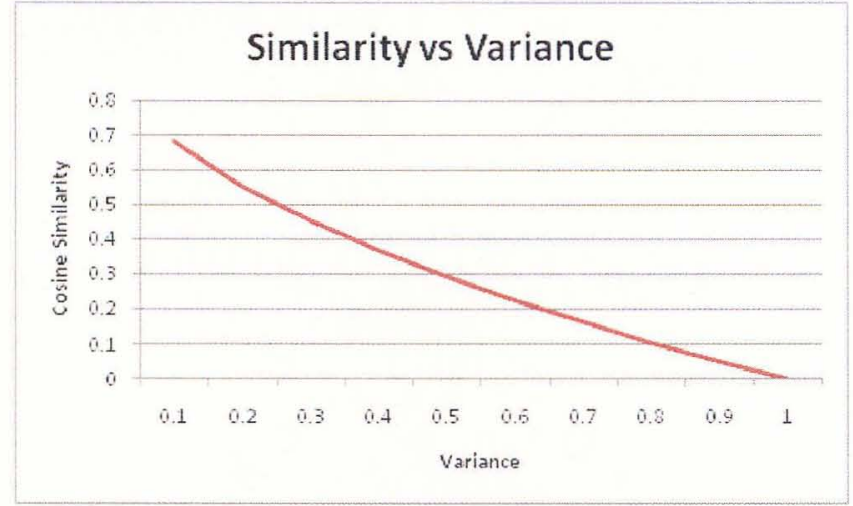

$$
\operatorname{Sim}_{\mathrm{Cos}}=1-\sqrt{d}
$$

or it can be written in terms of the profile variance:

$$
\operatorname{Sim}_{\mathrm{Cos}}=1-\sqrt{\sigma}
$$

Figure 4.1 shows the relationship between the profile variance and the cosine similarity. A low variance will result in high similarity between the session and the profile, hence higher quality. For example, a profile variance value of less than 0.1 will result in a cosine similarity close to 0.7 , which translates to the fact that the profile and the session share almost $70 \%$ of their content, which indicates a pretty good quality. A higher profile variance value like 0.5 will translate to only $30 \%$ of similarity between the profile and the session, which indicates lower quality.

\subsubsection{Profile Cardinality}

The profile cardinality is the number of sessions assigned to the corresponding cluster. A higher cardinality means that this profile is more popular and interesting. However a higher cardinality profile might also be a result of the "default" cluster 
that captures all the "noise" in the data, which are sessions that are too different to form any strong and consistent usage patterns. When sessions are assigned to clusters based on similarity, many of these sessions end up with 0 similarity to all clusters (i.e. non-matching sessions), and end up lumped to the last cluster by default. As a result, this big cluster also ends up with a very high variance. The fewer "good" clusters that are discovered, the more non-matching sessions will fit this category and the higher the cardinality of this cluster. The cardinality will further be normalized to a percentage of the sum of all profile cardinalities, and will be used as a heuristic when comparing profiles variances, because comparing profile variances with close cardinality makes more sense.

When the profile gets updated, its cardinality is increased to reflect the number of new sessions that matched the profile, as shown in Algorithm 2. Hence, the cardinality of a profile at any given time is the sum of sessions that the profile acquired up to and including that time period. The cardinality $N_{i}^{t}$ of a profile $P_{i}$ at time period $t$ can be written as follows

$$
N_{i}^{t}=\sum_{j=1}^{t} n_{i}^{j}
$$

where $n_{i}^{j}$ is the number of sessions identified to be close to the profile $i$ at time $j$. Furthermore, for evaluation purposes, the accumulated cardinality is normalized by the sum of all profile cardinalities at time period $t$. Thus, it can be defined as follows:

$$
\left(N_{i}^{t}\right)_{n o r m}=\frac{N_{i}^{t}}{\sum_{j=1}^{|P|} N_{j}^{t}}
$$

where $|P|$ is the total number of profiles.

\subsubsection{The matching vs. distinct sessions}

Capturing the changes in usage behavior is done by first updating the existing 
profiles based on the matching sessions from the new logs, and then discovering the patterns from only the new (distinct) usage sessions. The matching sessions refer to the sessions that matched an existing profile and are used to update the properties of that profile, while the distinct sessions are the ones that are not close enough to any of the existing profiles, and therefore they are used to extract new profiles.

The percentage of matching sessions will represent how restrictive the discovery process was. A high matching percentage (low distinct percentage) might indicate that sessions that are not very similar were used to update the profiles, which can lower the quality of these profiles. However, this is not the case all the time, because a very high quality profile might also match a large number of very similar sessions simply because of the distribution of sessions. Moreover, a larger number of matching sessions affects the performance of the pattern discovery algorithm, since only a smaller part of the original $\log$ file will undergo the re-discovery process. This is one of the major advantages of the proposed framework over the traditional discovery process of all logs at once.

\subsubsection{Number of profiles}

After each discovery process, some profiles are created and some are updated. Tracking these numbers can give an insight about which time periods have witnessed changes in usage behavior, and what were the trends. Three types of profiles are defined:

1. Discovered: a profile that is completely new, and was generated from the "distinct" sessions during the current batch or period.

2. Static: a profile that has already existed from the last time period, but was not updated by any new sessions in the current period. 
3. Updated: a profile that has already existed but was updated by the new "matching" sessions in the new period.

Tracking the number of profiles and their types over the evolution periods can reflect the overall usage trends of the users. A high number of discovered profiles may indicate that the usage patterns have drastically changed from previous times, which for example, would trigger the business need to capture these changes and develop marketing strategies to satisfy this new market.

A large number of static profiles might indicate that there was little change in the usage patterns, or that these profiles are becoming obsolete and no longer reflect the current activity. A large number of updated profiles is a good indication that the profiles are of good quality, since many sessions are still matching them.

However, making conclusions based only on the profile numbers and their types may be inaccurate, since the number of profiles and their types depend on the parameters used like the similarity threshold value. For example, a high threshold value would result in a larger number of discovered profiles and fewer updated profiles; whereas a lower value would increase the number of updated profiles.

\subsubsection{Profile Pair-wise Similarity}

A strong evaluation of the profile quality is to compare each profile with all other profiles, and determine if they are similar or not. A large number of very similar profiles indicates a poor clustering result, so our aim is a smaller number of similar profiles. The Binary Cosine Similarity defined in Eq.(3.6) will be used for comparing two profiles instead of a profile and a session. The similarity will be mapped to the dissimilarity measure defined in Eq.(3.7).

Since most profiles are not completely different from each other (they can share some URLs), a threshold value will be used to count the number of profiles that are too similar to each other. The value of 0.01 will be used as the threshold value, i.e. 
if the difference between two profiles is less than 0.01 then the profiles are considered similar. The value of 0.01 in the difference means a similarity of 0.9 between profiles as given in Eq.(4.1).

\subsubsection{Profile Density}

The profile density is another quality metric for describing profiles, that was defined in Section 3.4 as the sum of session weights divided by the profile variance. The weight of each session is now considered to be 1 (unlike the variance definition in Eq.(3.4)), since we are already restricting only very similar sessions to update the profile (thus outliers are eliminated). Hence, the profile density $\left(D_{i}^{t}\right)$ at time period $\mathrm{t}$ can be defined as follows:

$$
D_{i}^{t}=\frac{N_{i}^{t}}{\sigma_{i}^{2}}
$$

where $N_{i}^{t}$ is the profile cardinality (i.e. the number of sessions that are assigned to or have updated this profile) up to time period (t).

The profile quality generally increases as its variance decreases, as discussed in Section 4.1.1, and a higher cardinality is also desirable as discussed in Section 4.1.2. Hence, the profile density $D_{i}^{t}$ combines two quality metrics, and since it increases when cardinality is high and variance is low, then a high value for the density is desirable and indicates a high quality profile with high compactness (low variance) and more importance (high cardinality).

The advantage of using the density metric is that it considers two important quality metrics in combination. Using only the profile variance $\sigma_{i}^{2}$, alone, to judge the profile quality is not sufficient, since a profile with very few similar sessions might have low variance, but that is not necessarily desirable if profiles are to represent mass user patterns. Moreover, using only the cardinality $\left(N_{i}^{t}\right)$ is not accurate, because a 
large cardinality might be a result of the "default" cluster which acquires all nonmatching sessions regardless of their homogeneity.

\subsection{Parameter configuration}

The pattern discovery process depends on a number of parameters that affect the usage profiles and their quality. Varying the number and values of these parameters would help in determining the best configuration to be used, and help point out the weaknesses and strengths of the proposed framework. Only the most important parameters will be discussed in this thesis. Other parameters could be studied in the future.

\subsubsection{Method of discovery}

The first method of discovery is denoted as the "Evolution" mode, where different batches of data are processed and profile evolution is tracked through these periods. The second method is denoted as "Traditional" pattern discovery mode, which accepts all the web logs at once, and tries to discover the profiles in one shot. Therefore to emphasize the importance and scalability of the evolution mode discovery, the profiles discovered through "evolution" should be compared against traditional 1-shot profiles.

The profiles discovered in the traditional mode are expected to be of higher quality, since all log data are mined in one shot. Hence, each session will be compared to each other session during all time periods, and based on these similarities the profiles will be created. However, in the evolutionary mode, the session is only compared to all sessions in a single time period. The latter approach is more realistic since real website log data comes in batches with time (like data streams), and there is no way to know what the new logs would be. 


\subsubsection{Method of matching profiles}

This parameter determines which similarity measure is used when comparing a new session to existing profiles, and deciding whether this session is close enough to this profile. The two methods that will be used are the Binary Cosine Similarity defined in Eq.(3.4), and the Robust Weight Similarity which is defined in Eq.(3.5).

The Binary Cosine Similarity depends primarily on the number of common URLs between the profile and the session, whereas the Robust Weight Similarity goes further by being more sensitive to the profile's variance as well. A profile with lower variance (typically indicating high quality) is more restrictive in matching, so as to maintain its quality.

\subsubsection{Similarity threshold}

For both methods of finding the similarity between a profile and new sessions, a threshold value $\left(\operatorname{Sim}_{\min }\right)$, is used to control the strength of matching and thus the quality of resulting profiles. If the session is similar enough to a profile, i.e. the similarity is more than the threshold, then it will be used to update the profile (i.e. if $\operatorname{Sim}\left(S_{j}, P_{i}\right)>\operatorname{Sim}_{\text {min }}$ then update $\left.P_{i}\right)$.

Choosing the right threshold value $\left(\operatorname{Sim}_{\min }\right)$ can be done by trial and error. A higher threshold value leads to more restrictive and higher quality profiles. However, if it is too high, then too many "similar" sessions will fail to match existing profiles and will be forced to contribute to the re-discovery of new profiles. This in turn would lead to "duplicate" or redundant profiles that keep getting re-discovered. Choosing the threshold value is also domain-dependent. For example, an on-line encyclopedia might prefer more accurate and restrictive profiles than an on-line store, since an encyclopedia aims to direct users to accurate and specialized sources of information, while the on-line store would prefer the users to browse through more products even though they may be less similar to the initial product that the user was looking at 
(in hope of cross-selling).

For the Binary Cosine Similarity, two values are chosen: an average threshold of 0.3 and a more restrictive value of 0.5 . For the Robust Weight Similarity, two values are also chosen: an average value of 0.3 and a more restrictive value of 0.6 . These values were chosen based on some trial an error and historical results.

\subsubsection{URL weight threshold}

A URL significance weight threshold is used in two phases of the discovery process to make sure that only significant URLs are taken into account when comparing profiles. The first phase is in the post-processing phase in HUNC discussed in Section 3.5 , where profiles are generated from the clusters discovered during the pattern discovery process in Section 3.4. The threshold value is applied to make sure that only URLs that are significant enough in the cluster are selected in the final profiles. This is needed to filter out any weak URLs which might risk affecting the profile description.

The second phase where the URL significance weight is used, is in the profile updating algorithm (Algorithm 2), where only significant URLs in the profile are compared to the current sessions to calculate the Binary Cosine Similarity or the Robust Weight Similarity. This is also necessary to make sure that an accurate and reliable update is done on profiles. Despite thresholding URLs when creating profiles, weak URLs might still find their way into the profile if they were part of a lengthy session with many URLs, that is used to update the profile.

However, the use of a URL weight threshold may introduce a critical trade-off, because on the one hand, the threshold will help prevent infrequent URLs from affecting the updating of profiles, but on the other hand, it risks discarding important updates to the profiles in their premature stage (i.e. while still weak). This is because URLs may start with low weight, particularly relative to the rest of the well 
established URLs in the profile, and then might gain more weight over time, however using thresholding risks discarding them in the early steps.

As for the previous threshold values, choosing the right value is domain-dependent and can be done based on trial and error. Two URL weight threshold values are used in our experiments: an average value of 0.04 and a more restrictive value of 0.1 .

\subsection{Experimental Configuration}

The four parameters controlling the discovery of the usage patterns are shown in Table 4.2 with their different values. For the URL significance weight threshold, the term URL_TH will be used, for the Binary Cosine Similarity threshold, the term CosSim_ TH is used, and for the Robust Weight Similarity threshold, the term RobWT_ $\mathbf{T H}$ is used. Each parameter configuration will generate an experiment and its resulting evaluation metrics.

Table 4.2: Experimental Configuration

\begin{tabular}{|c|c|c|c|c|}
\hline Method & Matching Criteria & URL_TH & CosSim_TH & RobWT_TH \\
\hline \hline Evolution & Binary Cosine & 0.04 & 0.3 & - \\
\hline & & 0.04 & 0.5 & - \\
\hline & & 0.1 & 0.3 & - \\
\hline & & 0.1 & 0.5 & - \\
\hline & Robust Weight & 0.04 & - & 0.3 \\
\hline & & 0.04 & - & 0.6 \\
\hline & & 0.1 & - & 0.3 \\
\hline & & 0.1 & - & 0.6 \\
\hline Traditional & - & - & - & - \\
\hline
\end{tabular}

The HUNC algorithm is used to discover the profiles from distinct sessions. Table 4.3 shows all the HUNC parameters and their values. Changing the values of these parameters might affect the resulting profiles quality. But they were chosen based 
Table 4.3: HUNC Parameters

\begin{tabular}{|c|r|c|c|}
\hline Parameter & The smallest allowable size of a cluster \\
\hline Min. Card. & If cardinality of a cluster is less than this, it will not be split & Value \\
\hline Min. Card. to Split & If cluster's variance is less than this, it will not be split \\
\hline Var. Threshold to Split & Factor of variance used as threshold for within niche distance in mating restriction \\
\hline Var. Factor & Threshold to identify valid individuals when restricting mating of good individuals from different niches & 1000 \\
\hline Min. Fitness Crossover & Maximum number of levels of hierarchical clustering \\
\hline Max. Num. of Levels & The initial population size used in the genetic algorithm \\
\hline Population Size & The probability of performing crossover (per pair of individuals) \\
\hline Crossover Probability & The probability of any bit of a chromosome being mutated \\
\hline Mutation Probability & & 0.5 \\
\hline
\end{tabular}

on previous experiments on many website logs. Since this thesis aims to study the changing usage behavior over the web, and not the performance of HUNC, these parameter values will be the default values for all experiments.

\subsection{Experiment 1: University of Missouri}

\subsubsection{The Dataset}

This log data was collected from the CECS Department's website of the University of Missouri during a two-week period, from January 22nd 1998 until February 4th 1998. There were 32,770 access requests done during that period, grouped into 1704 sessions. To track the usage changes, the logs were divided into four batches:

- Thursday, Jan 22nd - Sunday, Jan 25th : 7,015 access requests (21.41\%), 326 sessions $(19.13 \%)$

- Monday, Jan 26th - Friday, Jan30: 15,126 access requests (46.16\%), 746 sessions $(43.78 \%)$

- Saturday, Jan31-Sunday, Feb1: 2,972 access requests (9.1\%), 197 sessions (11.56\%)

- Monday, Feb 2nd - Wednesday, Feb 4th : 7,657 access requests (23.33\%), 435 sessions $(25.53 \%)$ 
The data was cleaned during the preprocessing phase as described in Section 2.4.1. Figure 4.2 shows the percentage of "bad access" and "good access" requests. Bad access requests include irrelevant and noise requests from search engines requests and requests that resulted in an erroneous status code. Good access requests are the remaining non-error generating requests.

Figure 4.2, shows the importance of the cleaning steps in the preprocessing phase, because all these bad requests would have adversely affected the profile discovery. Typically graphics requests may amount to 3-4 graphics per page or more (e.g. background picture, top banners, etc), that is why they end up being the majority of requests.

Figure 4.2: Missouri: Access Requests

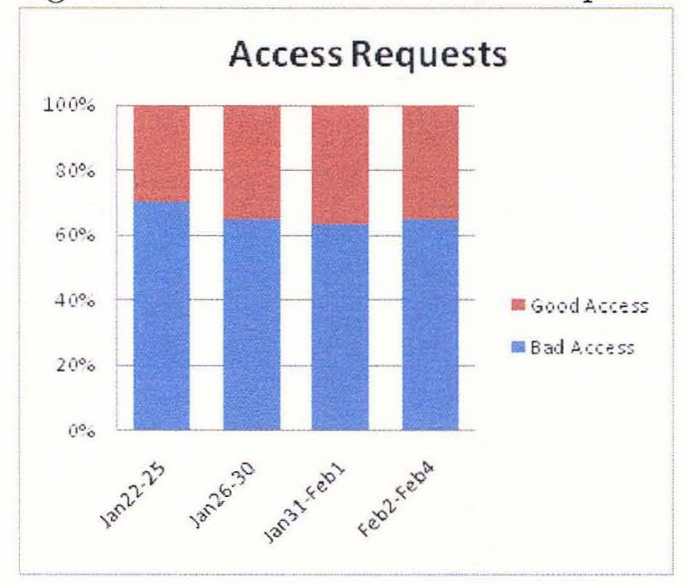

Table 4.4 shows the list of all profiles (after processing the last batch (Feb2-Feb4)) that resulted from mining this dataset. These profiles are the result of using Robust Weight Similarity with threshold value of 0.6 , and URL threshold value of 0.1 . The second column shows the total number of URLs in each profile, however for illustration purposes, only URLs with weight greater than 0.1 are shown. URLs were sorted in a descending order by the URL significance weight. 
Table 4.4: Missouri: Final Profiles for RobWT (0.6) and URL TH(0.1)

\begin{tabular}{|c|c|c|c|c|}
\hline Profile & Urit: & Card & Variance & Content \\
\hline 0 & 22 & 63 & 0.1542 & $\begin{array}{l}\text { /courses_index.html,/courses100.html, /courses.html, /, /cecs_computer.class, /courses200.html, /courses300.html, } \\
\text { /courses_webpg.html, /degrees.html, }\end{array}$ \\
\hline 1 & 29 & 68 & 0.230874 & $\begin{array}{l}\text { /cecs_computer.class, /people_index.html, /people.html, /faculty.html, /degrees.html, /grad_people.html, /staff.html, } \\
\text { /degrees_grad.html, }\end{array}$ \\
\hline 2 & 40 & 46 & 0.268294 & $\begin{array}{l}\text { / shi/cecs345, / shi, /,/people.html, /people_index.html, /faculty.html, / shi/cecs345/Lectures/04.html, /cecs_computer.class } \\
/ \sim \text { shi/cecs345/Projects/1.html, }\end{array}$ \\
\hline 3 & 231 & 223 & 0.592878 & $\begin{array}{l}\text { / c697168/cecs227/left.html, / c697168/cecs227,/ c697168/cecs227/head.html, / c697168/cecs227/main.html, } \\
/ \sim c 697168 / \operatorname{cecs} 227 / \text { handouts.html, }\end{array}$ \\
\hline 4 & 22 & 57 & 0.116559 & $\begin{array}{l}\text { /courses.html, /courses_index.html, /courses100.html, /,/cecs_computer.class, /people.html, /people_index.html, } \\
\text { /faculty.html, /general.html, /index.html, }\end{array}$ \\
\hline 5 & 19 & 68 & 0.165445 & $\begin{array}{l}\text { /courses_index.html, /courses } 100 . h t m l, / \text { courses.html, /,/cecs_computer.class, /courses300.html, /courses } 200 . h t m l, \\
\text { /courses_webpg.html, /courses } 400 . \mathrm{html} \text {, }\end{array}$ \\
\hline 6 & 28 & 52 & 0.193953 & $\begin{array}{l}\text { /people_index.html, /people.html, /faculty.html,/,/cecs_computer.class, /grad_people.html, /staff.html, } \\
\text { /undergrad_people.html,/research.html, }\end{array}$ \\
\hline 7 & 9 & 78 & 0.115836 & /./cecs_computer.class, /research.html, / searc, /degrees.html, /general.html, /general_index.html, /facts.html, \\
\hline 8 & 13 & 88 & 0.079423 & $\begin{array}{l}\text { / joshi/courses/cecs35, / joshi/courses/cecs352/slides-index.html, / joshi, / joshi/courses/cecs352/environment.html, } \\
/ \sim \text { joshi/courses/cecs352/outline.html, }\end{array}$ \\
\hline 9 & 10 & 38 & 0.110454 & 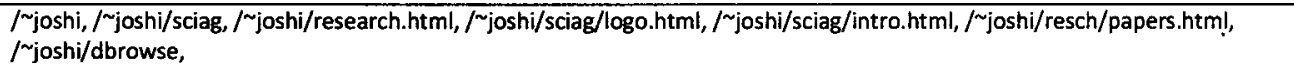 \\
\hline 10 & 53 & 36 & 0.325243 & $\begin{array}{l}\text { /faculty/springer.html, /people_index.html, /faculty.html, /people.html, /faculty/keller.html, /faculty/chen.html, } \\
\text { /faculty/plummer.html, /faculty/palani.html, }\end{array}$ \\
\hline 11 & 12 & 53 & 0.1091 & $\begin{array}{l}\text { / shi/cecs345, / shi/cecs345/java_examples, / shi/cecs345/Lectures/06.html, / shi/cecs345/Lectures/07.html, } \\
/ \sim \text { shi/cecs345/Lectures/05.html, }\end{array}$ \\
\hline 12 & 3 & 49 & 0.016306 & / yshang/CECS341.html, / yshang/W98CECS345, / yshang, \\
\hline 13 & 19 & 44 & 0.0902 & $\begin{array}{l}\text { / saab/cecs333,/ saab/cecs333/private,/ saab/cecs333/private/lecture_programs, } \\
\sim \text { saab/cecs333/private/textbook_programs, / saab/cecs333/final.html, }\end{array}$ \\
\hline 14 & 17 & 48 & 0.051113 & $\begin{array}{l}\text { / c697168/cecs227,/ c697168/cecs227/main.html,/ c697168/cecs227/left.html,/ c697168/cecs227/head.html, } \\
/ \sim c 697168 / \operatorname{cecs} 227 / \text { labs/main.html, }\end{array}$ \\
\hline 15 & 4 & 28 & 0.0218 & / manager/LAB/motif.html, / manager/LAB/tin.html, \\
\hline 16 & 6 & 82 & 0.556638 & /access, /access/details.html, \\
\hline 17 & 24 & 49 & 0.143885 & $/ \sim$ saab/cecs333/private, $/ \sim$ saab/cecs333, / saab/cecs333/private/assignments, $/ \sim$ saab/cecs333/private/lecture_programs, \\
\hline 18 & 17 & 61 & 0.2141 & $\begin{array}{l}\text { /cecs_computer.class, /courses.html, /courses100.html,/courses_index.html, /degrees.html, /degrees_undergrad.html, } \\
\text { /degrees_index.html, /bsce.html, }\end{array}$ \\
\hline 19 & 30 & 110 & 0.708612 & $/ \sim$ joshi/courses/cecs352, / c697168/cecs227, \\
\hline 20 & 8 & 56 & 0.242 & $\begin{array}{l}\text { / joshi/courses/cecs352,/ /joshi, / joshi/courses/cecs352/proj/overview.html, / joshi/courses/cecs352/proj, } \\
\text { / joshi/courses/cecs352/slides-index.html, / joshi/courses/cecs352/outline.html, }\end{array}$ \\
\hline 21 & 9 & 57 & 0.1266 & $\begin{array}{l}/ \sim \text { shi/cecs345, / shi/cecs345/java_examples, } / \sim \text { shi/cecs345/references.html, } / \sim \text { shi/cecs345/Lectures/07.html, } / \sim \text { shi, } \\
/ \sim \text { shi/cecs345/Lectures/09.html, }\end{array}$ \\
\hline 22 & 8 & 250 & 0.8325 & $\begin{array}{l}\text { /,/cecs_computer.class, / } \sim \text { saab/cecs } 333 / \text { private, / } \sim \text { saab/cecs333,/ } \sim \text { saab/cecs333/private/assignments, /courses. html, } \\
\text { /courses_index.html, /courses100.html, }\end{array}$ \\
\hline
\end{tabular}




\subsubsection{Profile Variances}

To study how the profiles variances change over time, and hence how their quality changes, the variance for each profile in each time period was plotted against time, in Fig.4.3 when using Binary Cosine Similarity to match profiles with sessions. A plot for each different configuration is used. The profile numbers in the legend are ordered (in a descending order) based on their cardinality percentage (i.e. the cardinality of the profile divided by the total cardinalities of all profiles at the last batch).

A low profile variance is desirable since it means that its assigned sessions are closer to the profile. Hence, if the profile variance decreases over time, then this profile can be considered to be improving and gaining quality. Fig.4.3(a) shows that profiles are generally decreasing in variance over time, especially the ones with higher cardinality (such as profiles 11,15 , and 3 ). This means that as these profiles capture more sessions, and they improve in quality over time. Profile number 19 seems to have high variance and high cardinality which is not desirable, however it was discovered only at the last time period, so over time, it might get improved. The low-cardinality profiles (like 7, 9 and 14) show an undesirable behavior, which is an increase in their variance, however these represent only a small portion of the sessions, so they are naturally sensitive to even small changes resulting from a few new sessions.

Fig.4.3(b) also shows a similar behavior to Fig.4.3(a) with the difference that the number of profiles with high cardinality and high variance is smaller. Thus, the majority of profiles have lower variance which is more desired. Thus, using a more restrictive URL weight threshold (0.1) before matching resulted in better quality profiles (as expected).

Figs.4.3(c) and (d) show similar behavior where the profile variances does not change much over time. However, the main difference was in the total number of profiles generated. Fig.4.3(c) has more profiles than Fig.4.3(d). So changing the URL threshold didn't affect the quality as much when using a more restrictive similarity 
threshold of 0.5. The majority of profiles in Fig.4.3(c) and (d) have low variance which is good, but they are not improving over time, which means that these configurations are highly sensitive to the initial profiles, in contrast to the configurations in Figs.4.3(a) and (b) where variances did change over time. This is probably because the high similarity threshold makes it very hard for the new period's session to "match" the existing profiles. Thus they remain more stable compared to when lower similarity thresholds were used.

Figure 4.3: Missouri: Evolution of Profile Variances (Binary Cosine Similarity)

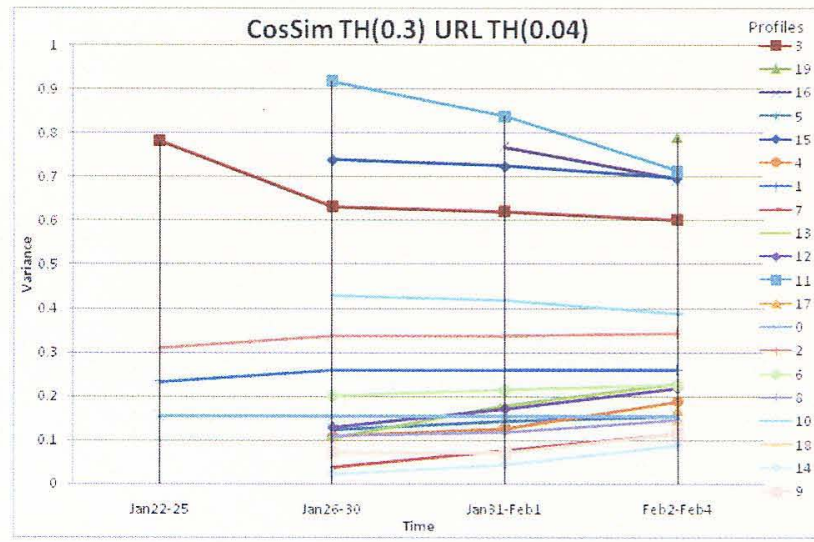

(a)

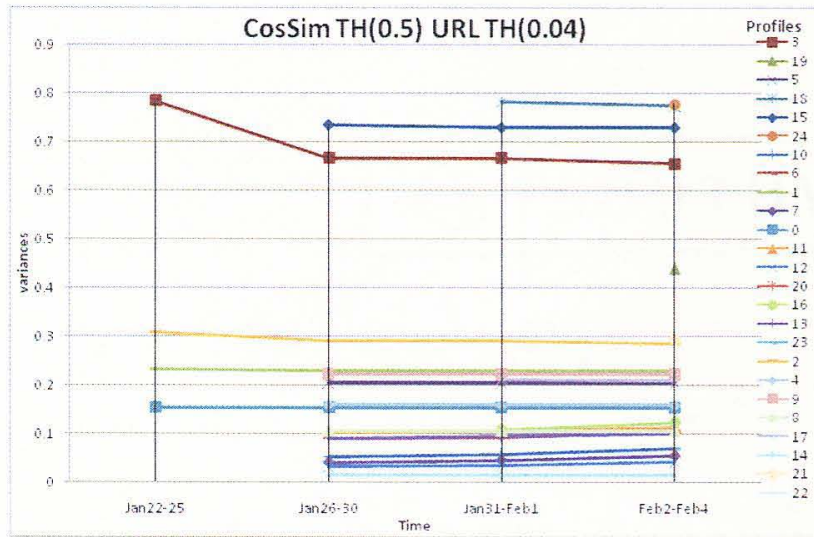

(c)

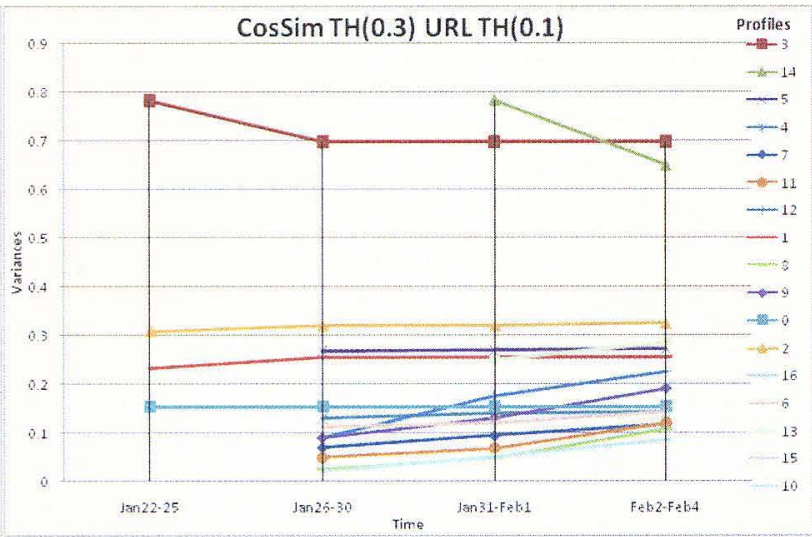

(b)

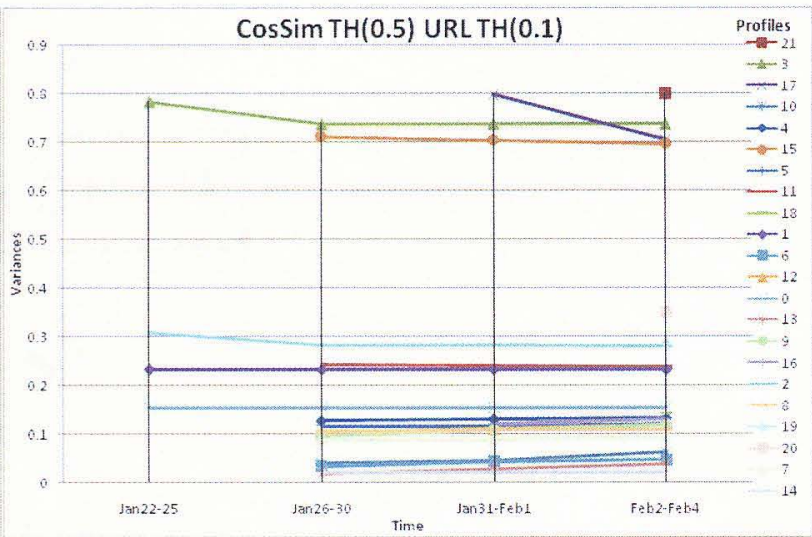

(d)

Fig.4.4 shows the profile variances over time when using the Robust Weight Similarity. Figs.4.4(a) and (b) show a low number of discovered profiles, low overall 
variance, and stable variance over time. A low number of profiles means less diversity (i.e. more general profiles). So the change in the URL threshold did not seem to affect the variances, it only changed the number of discovered profiles. This seems to be a different behavior from using the Binary Cosine similarity where changing the URL threshold resulted in better quality profiles.

Figs.4.4(c) and (d) show a similar behavior to Figs.4.3(a) and (b), where the profile variances showed improvement over time. However, they have the advantage that even the lower cardinality profiles retain a constant variance over time. This desirable behavior is due to the fact that the Robust Weight Similarity is more sensitive to the profile variance, i.e. it is naturally more restrictive when the profile has lower variance, thus "shielding" even the vulnerable small profiles from noise.

Using a more strict Robust Weight Similarity threshold as shown in Figs.4.4(c) and (d) has resulted in more profiles than in Figs.4.4(a) and (b), i.e. more "detailed profiles". Another conclusion can be drawn from this figure is that the URL threshold value did not really affect the quality when using the robust weight threshold, whereas it had more effect when the cosine similarity was used. So based only on Fig.4.3and Fig.4.4, using the more restrictive Robust Weight threshold value has resulted in higher quality and detailed profiles. However we need to also look at the "rest" of the metrics like the inter-profile similarity before making a judgment.

To study the overall quality of the profiles, some aggregate metrics were calculated during all the time periods. Fig.4.5 shows the minimum, maximum, average, and median variances of the profiles at the end of evolution: Fig.4.5(a) shows the aggregates when using the Binary Cosine Similarity, while Fig.4.5(b) shows the aggregates for Robust Weight Similarity.

The overall trend regarding the aggregates in both charts seems to be similar, where the average and the median variances maintained similar levels. However the 
Figure 4.4: Missouri: Evolution of Profile Variances (Robust Weight Similarity)

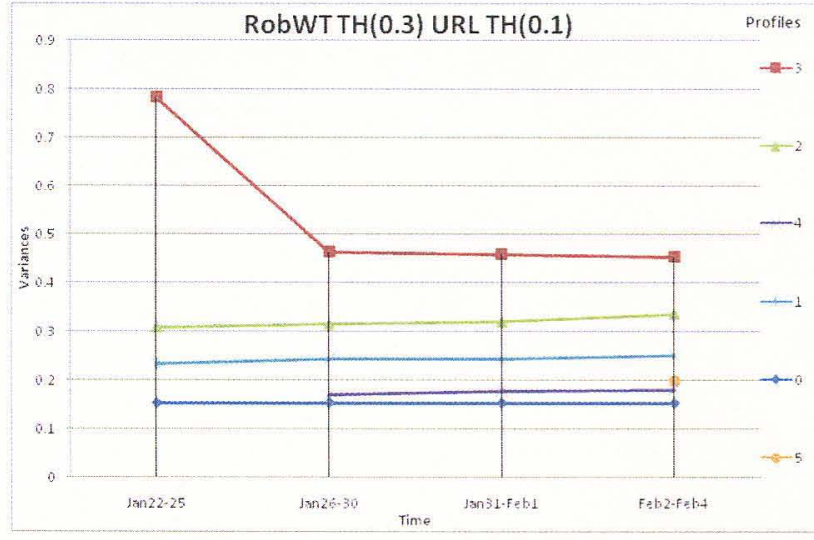

(a) (c)

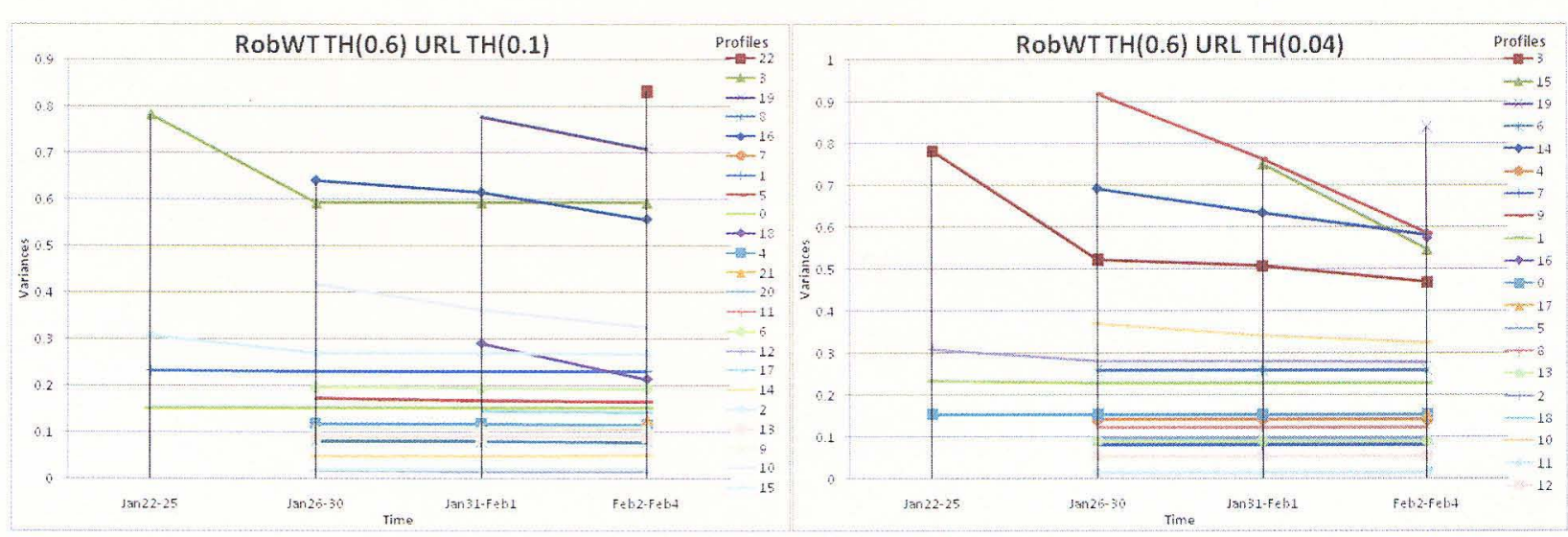

RobWT TH(0.3) URL TH(0.04) Profiles

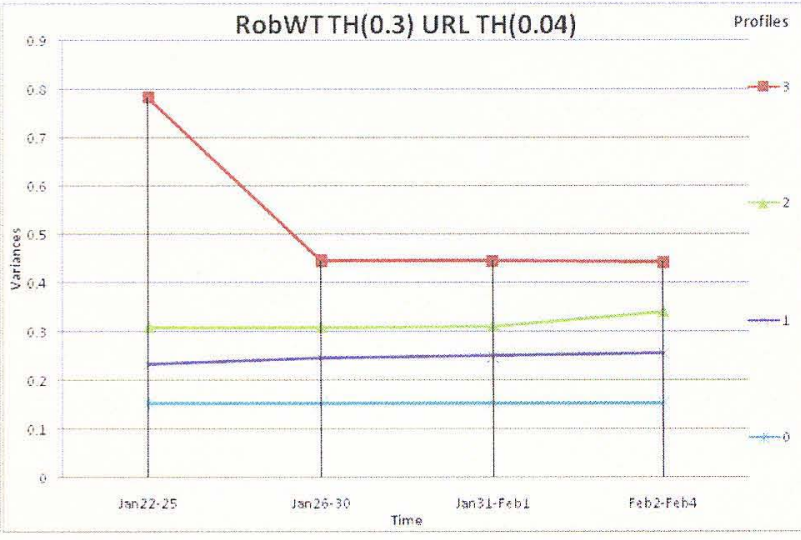

(b)

(d)

maximum variance decreased significantly when the similarity threshold was lower (0.3 in both CosSim and RobWT), and increased when the similarity threshold was stricter (i.e. using higher values of 0.5 in CosSim and 0.6 in RobWT for matching new sessions to old profiles). A general conclusion can be drawn that the URL weight threshold did not affect the aggregate variance metrics. In contrast to the maximum variance, the minimum, median and average variance seemed to decrease with stricter matching thresholds. Hence, a stricter matching results in an increase in the maximum variance and decrease in the minimum variance. This can be explained by the fact that stricter matching allows only "very" similar sessions to update most "good" profiles, resulting in profiles of better quality (lower variance). However, this 
Figure 4.5: Missouri: Profiles Sigma Aggregates

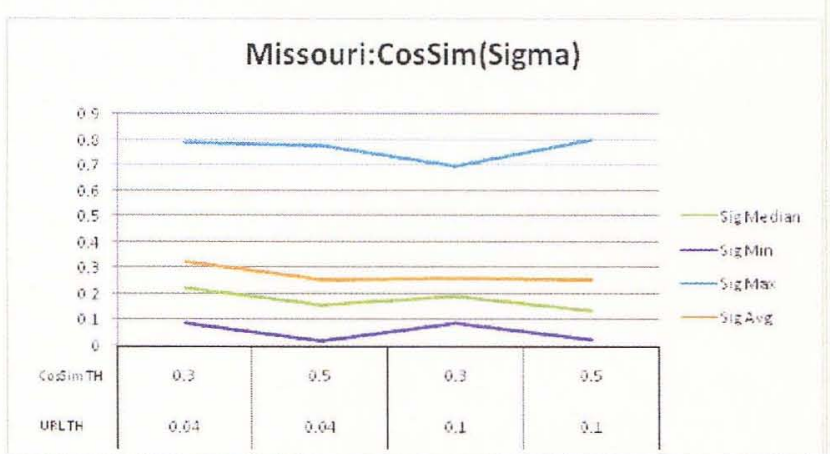

(a)

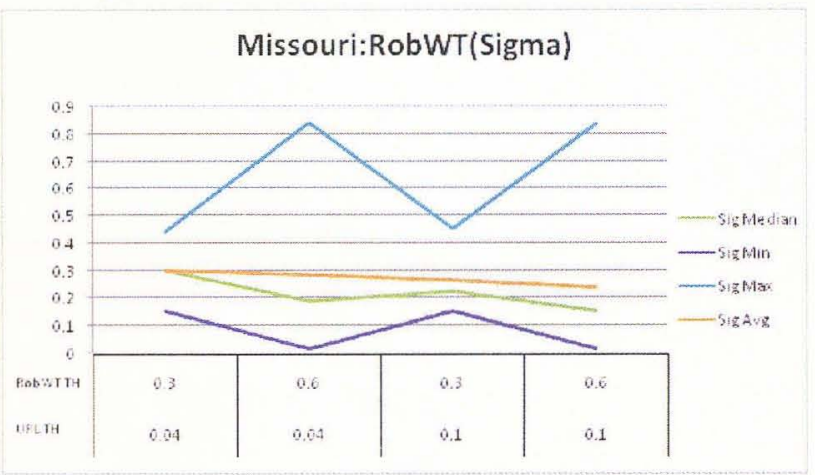

(b)

increased strictness will cause more new sessions to fail in matching most profiles and thus end up in the big "default" cluster that acquires all the heterogeneous sessions, which tends to have maximum variance, thus increasing its variance even more.

The difference between the two results in Fig.4.5 is in the value of the maximum variance; while the minimum, average, and median variances change very little (Fig.4.5(a) has a narrow range of values for the maximum variance (0.7-0.8) whereas Fig.4.5(b) has a wider range $(0.4-0.8))$. This means that using the Robust Weight similarity can result in a set of profiles where the maximum variance is around 0.4 (when the similarity threshold is 0.3 ), so all the profiles are essentially of good quality. However, going back to Fig.4.4, the profiles generated in this case were too general , i.e. only a few generic profiles were created, and they maintained their variance over time which makes them more sensitive to the initial profiles discovered.

\subsubsection{Profile Evolution}

The number and type of profiles discovered during the evolution gives an overall picture of the changes in behavior trends. Distinct or newly discovered profiles indicate a radical change in browsing behavior, while static profiles indicate low traffic 
or obsolete profiles, and updated profiles indicate more stable representative profiles and/or similar browsing behavior by users.

Fig.4.6 shows the percentages of each type of profiles for each time period during evolution when using the Binary Cosine Similarity for matching. The initial time period (period 1) always results in all distinct profiles since there are no profiles from previous time periods.

During period 2 (Jan26-Jan30), the profile percentages are almost the same for all configurations. Almost $80 \%$ of profiles are distinct which means that the usage behavior in this period may have changed by $80 \%$. This might be due to many reasons: the initial data from Jan22-Jan25 was from Thursday to Sunday, with half of it on a weekend, so the traffic was low, and it might not be the best initial period to use. However, over time, profiles will evolve and eventually represent more typical everyday web usage patterns.

During the weekend in period 3 (Jan31 and Feb01), the traffic was the least, and the profile percentages were almost the same for two configurations Fig.4.6(b) and (d), where about $40 \%$ of the profiles remained unchanged because of low traffic, while $40 \%$ of the profiles got updated, and only $20 \%$ of the profiles were newly discovered. In contrast, the configuration in Fig.4.6(a) shows that about $20 \%$ of profiles are static, $75 \%$ got updated and $5 \%$ were newly discovered. The large number of updated profiles could be explained by the lower threshold value for similarity, which allows more sessions to be matched to existing profiles and cause more profiles to be updated. Fig.4.6(c) is similar to Fig.4.6(b) and (d) but with fewer static profiles.

At the end of evolution (period 4), the changes between different configurations became more apparent. Fig.4.6(a) has the highest percentage of updated profiles (about $80 \%$ ) due to the lower similarity threshold (0.3). Fig.4.6(b) has more balanced profiles because it has a stricter similarity threshold. Fig.4.6(c) and (d) are similar to each other where the updated profiles are about $60 \%$. Moving from Fig.4.6(c) to 
Figure 4.6: Missouri: Profile Counts (Binary Cosine Similarity)

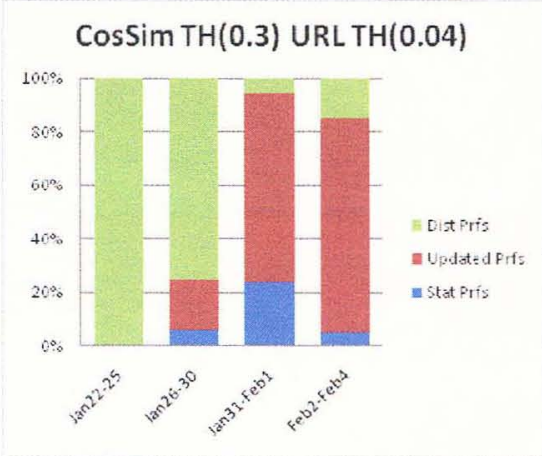

(a)

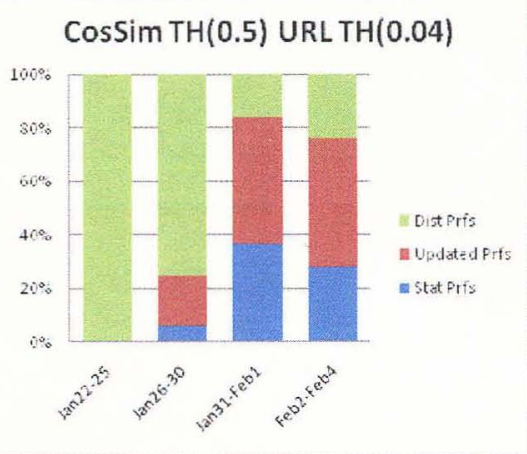

(b)

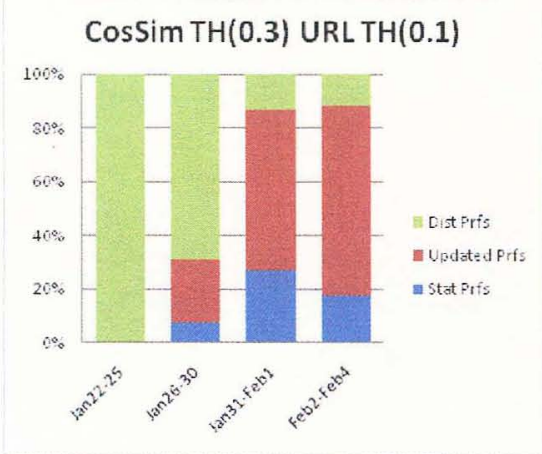

(c)

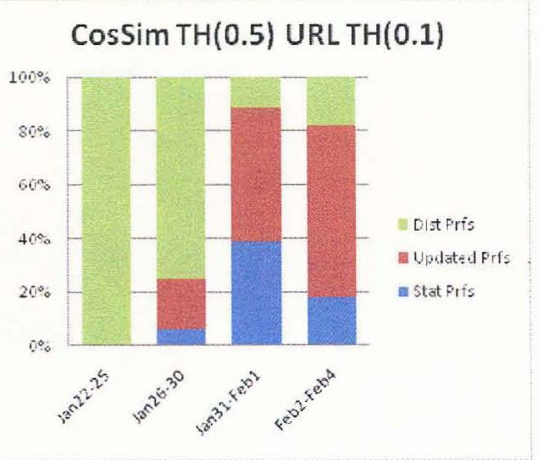

(d)

(d), the similarity threshold gets more restricted. However the percentage of updated profiles stayed the same which is the opposite of what happened with Fig.4.6(a). But this behavior can be explained by the more strict URL threshold, which ensures that higher quality profiles are generated, and hence even if the similarity threshold is loose, weak sessions will not be matched to these profiles.

Fig.4.7 shows the percentages of profile evolution when the Robust Weight Similarity was used. The profiles show more dynamic changes in profiles percentages compared to Fig.4.6that used the Binary Cosine Similarity.

Fig.4.7(a) shows that the percentages of profiles are the same for all time periods (except the initial time since they were all distinct). Using this configuration, only 
four profiles were developed: one of them remained the same, while the other three were updated during all time periods. The low similarity threshold is the reason for having a low number of profiles, because a new session can easily be matched to any of the existing profiles, and hence old profiles get updated instead of new profiles being discovered. The variances for these profiles are low as shown in Fig.4.4, but they are more general, and sensitive to initial data.

Fig.4.7(c) has also resulted in a low number of profiles (6 profiles), but some profiles were discovered during the evolution such as in period 2 (Jan26-Jan30). So even though the similarity threshold was low, the restricted (compared to Fig.4.7(a)) URL weight threshold caused some of the sessions to not match the existing profiles, and hence created more new distinct profiles.

Fig.4.7(b) and (d) are close in their behavior and they have many more profiles developed than previous configurations. During the second time period, about $75 \%$ of profiles were newly discovered, which is similar to Fig.4.6(b) and (d), and can also be explained for the same reasons, such as low traffic during weekend in the initial data. In the next time period more than $60 \%$ profiles remained static, which is also because that traffic was during the week end. The last time period witnessed more updated profiles and some static and distinct profiles, which is more typical of usage behavior and means that profiles are of good quality.

\subsubsection{Matching vs. Distinct Sessions}

The percentage of sessions that matched profiles to the sessions that were not close enough to any of the profiles is an important indication about how strict the matching process was. Fig. 4.8 shows the percentage of matching sessions during all evolution period for both similarities matching methods (CosSim and RobWT). For 
Figure 4.7: Missouri: Profile Evolution (Robust Weight Similarity)

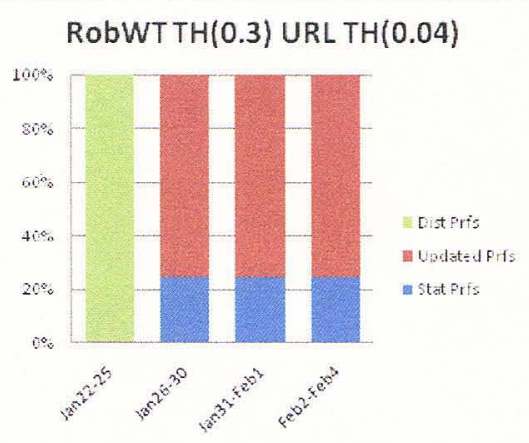

(a)

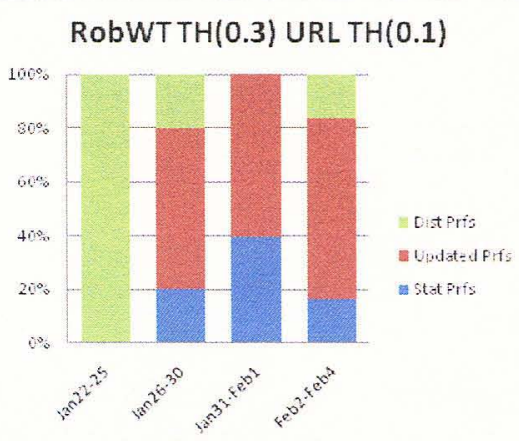

(c)

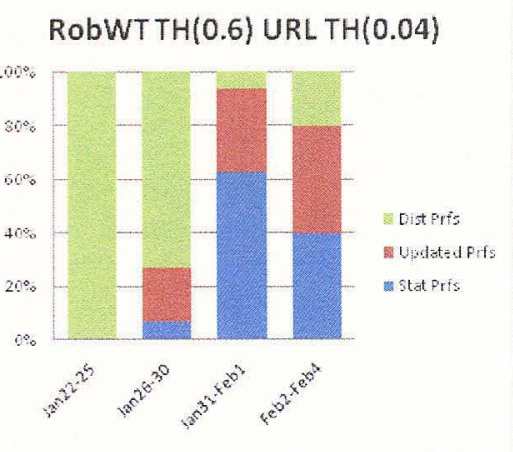

(b)

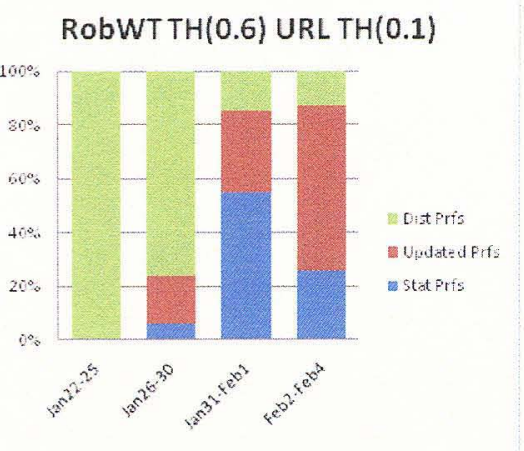

(d) 
simplicity, the similarity threshold was joined on one axis for the two methods of matching.

A general trend for both methods is that the percentage of matched sessions is high when the similarity threshold is low (0.3), and is low when a stricter threshold is used ( 0.5 for CosSim and 0.6 for RobWT). This has an intuitive explanation, which is that a lower similarity threshold allows more sessions to be matched with existing profiles. Another thing to notice for both methods is that the URL threshold value had a minimum affect on the matching percentage: when using 0.1 as URL threshold the percentage is slightly decreased compared to using 0.04 . So in general, the URL weight threshold is not significant when it comes to sessions matching percentage.

The RobWT method causes more changes in the percentage of matching sessions, when changing the similarity threshold, than the CosSim does. In fact, it goes from $80 \%$ when using 0.3 as threshold to only $15 \%$ when using a 0.6 threshold. In contrast, CosSim went from $20 \%$ when using 0.3 threshold to about $9 \%$ when using 0.5 threshold. This is also due to the sensitivity of RobWT to the profile variance. Since using both threshold values for RobWT result in overall low variance profiles as previously shown in Fig.4.4, these profiles would show more resistance to accepting different sessions, and hence, for a high similarity threshold of 0.6 , they will not match new sessions as easily as the CosSim would.

A more detailed view of the distinct and matching sessions is seen in Fig.4.9, where the percentages of both the distinct and matching sessions are shown during all time periods for each parameter configuration, when using the Binary Cosine Similarity. It can easily be seen that the number of matching sessions is the highest in Fig.4.9(a) and (c), and less in Fig.4.9(b) and (d). This is due to the loose similarity threshold used in the former, and the strict similarity threshold in the latter. A higher threshold 
Figure 4.8: Missouri: Matching Sessions Percentage

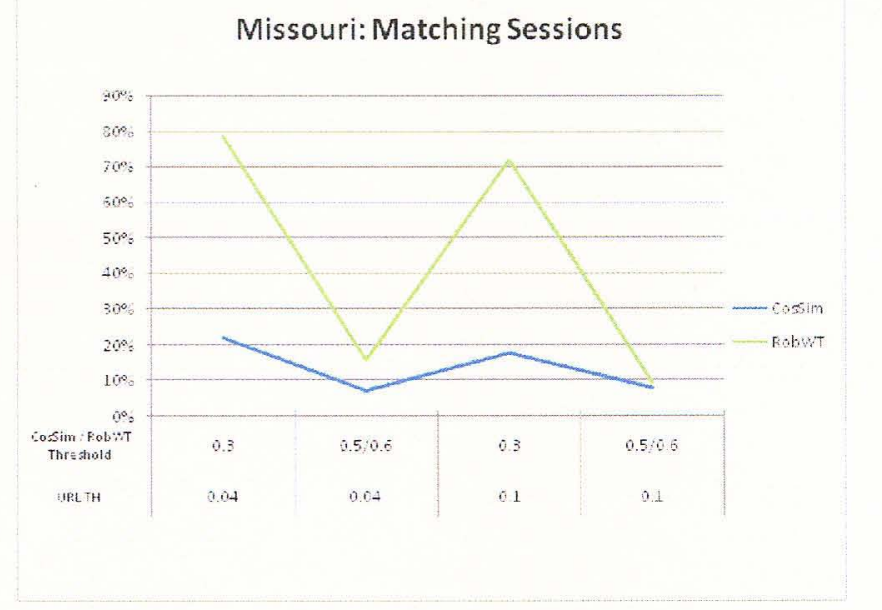

means that the sessions should really be closer to the profiles in order to update them, so fewer sessions would match profiles.

Another observation is that the time period 3 (Jan31-Feb1) witnessed the highest percentage of matching sessions relative to other time periods. This time period falls in a weekend, so the traffic in general is lower than week days so, given that good profiles have already been developed, the browsing behavior would match more profiles than it would in regular traffic.

Fig.4.10 shows the percentages of missing and matching sessions when using Robust Weight Similarity. Fig.4.10(a) and (c) are similar, and they show that the majority of sessions match the profiles and cause their update. Whereas in Fig.4.10(b) and (d), the majority of sessions did not match any of the profiles, which results in creating new profiles. The stricter similarity threshold (0.6) in Fig.4.10(b) and (d) makes it hard for new sessions to match the profiles, which in turn results in the high percentage of distinct sessions. 
Figure 4.9: Missouri: Missing vs. Distinct Sessions (Binary Cosine Similarity)

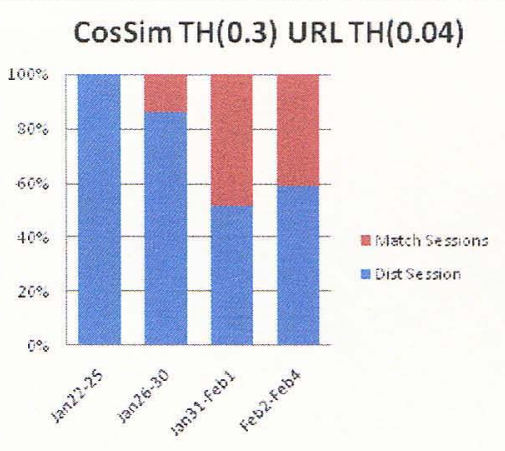

(a)
CosSim TH(0.5) URL TH(0.04)

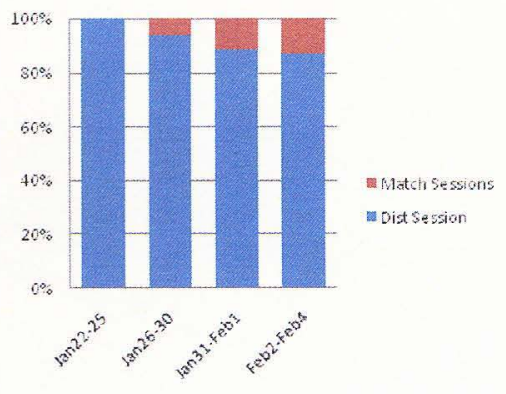

(b)

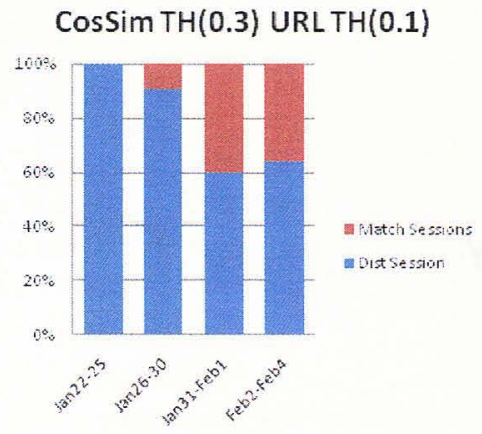

(c)
CosSim TH(0.5) URLTH(0.1)

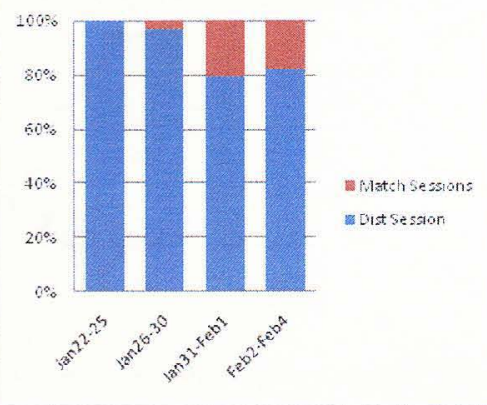

(d) 
Figure 4.10: Missouri: Missing vs. Distinct Sessions (Robust Weight Similarity)

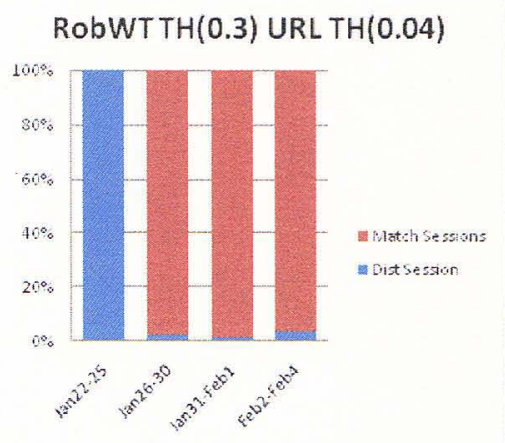

(a)

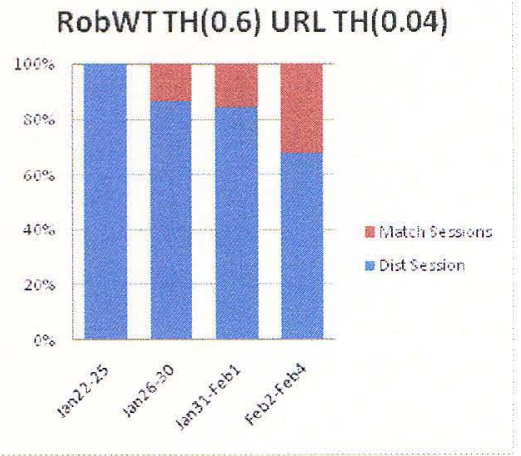

(b)

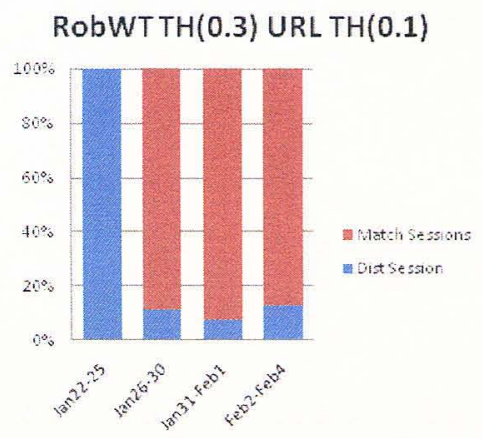

(c)

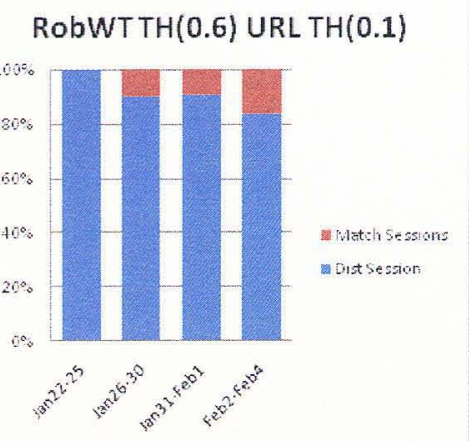

(d) 


\subsubsection{Profile Cardinality}

The profile cardinality is the number of sessions that the profile represents. A higher cardinality means that the profile is more popular and represents more usage behavior. To compare the cardinalities of different profiles, the percentage of each profile's cardinality with respect to the total number of sessions is used.

Fig.4.11 shows the cardinality percentage of both the profiles with maximum and minimum variance for the different parameter configurations. The cardinality percentage of the profile with minimum cardinality stays the same regardless of the different configurations. Recall that the profile with minimum variance is the highest quality (most compact) profile, so it is desirable to have it with high cardinality, however in this dataset its cardinality is low.

For the profile with maximum variance, the trends in both charts seem similar, where the cardinality percentage is higher when the similarity threshold is more restrictive. However, the Robust Weight threshold causes a more drastic change in the cardinality percentage than the Binary Cosine threshold. This can be explained by the fact that when the RobWT threshold is 0.3 only a few profiles are created, thus forcing one of them (the default profile) acquire all the sessions that do not match any profile.

Another thing to notice is that in Fig.4.11(a), the cardinality percentage of the profile with maximum variance is higher when the URL weight threshold is higher (more restrictive), whereas in Fig.4.11(b), the URL weight threshold does not seem to have a big effect.

To show how the profiles are increasing or decreasing in their quality, the number of sessions assigned to the profile at each time period is tracked. Fig.4.12 shows the cardinality percentage of each profile at each time period with the Binary Cosine 
Figure 4.11: Missouri: Cardinality of Max and Min Sigma

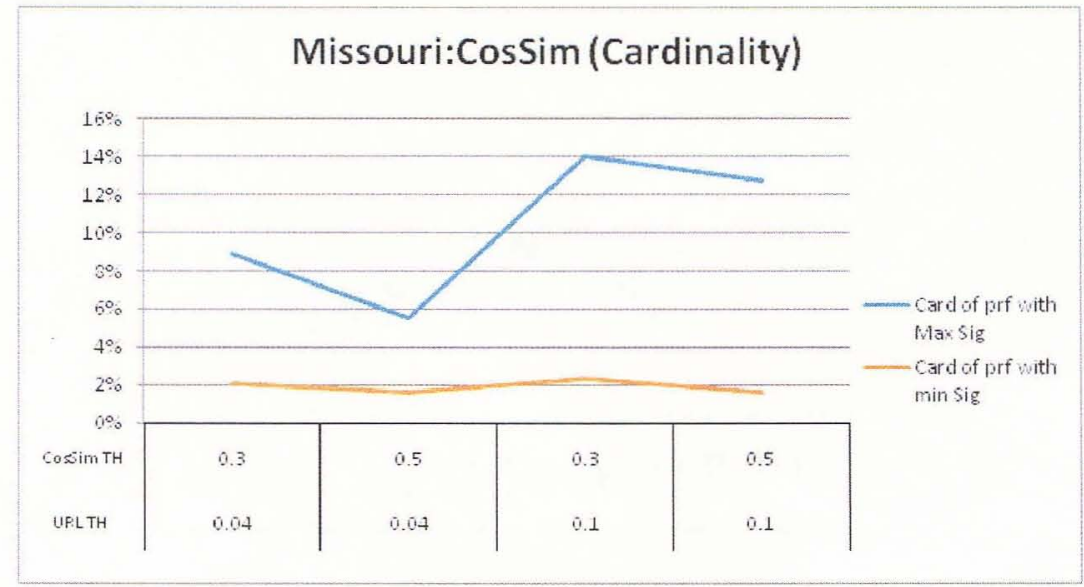

(a)

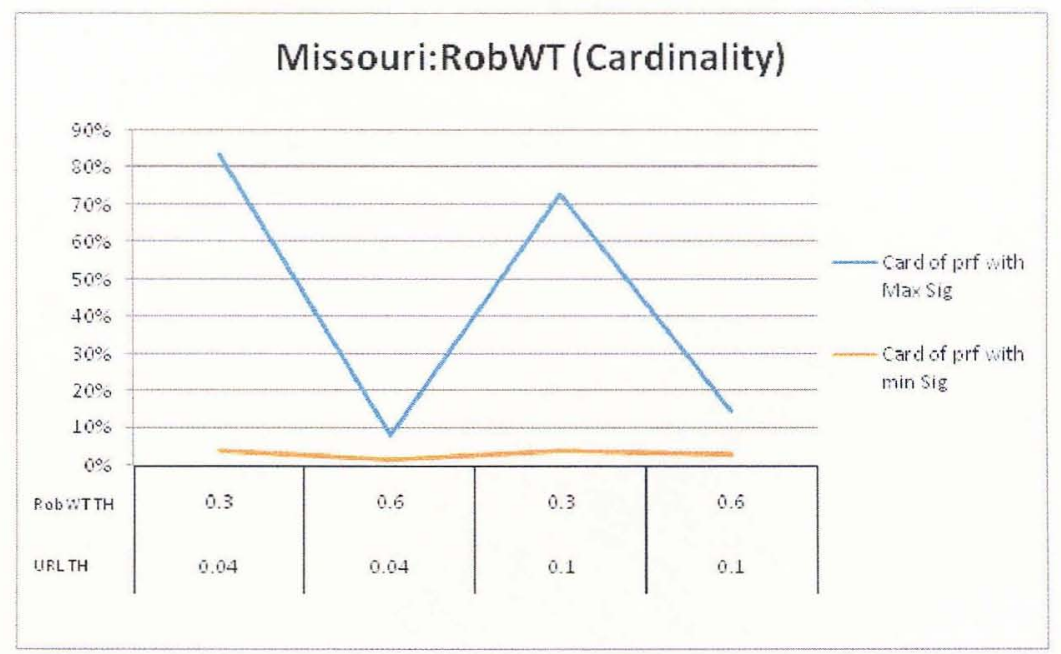

(b) 
Figure 4.12: Missouri: Evolution of Profile Cardinality Percentages (CosSim)

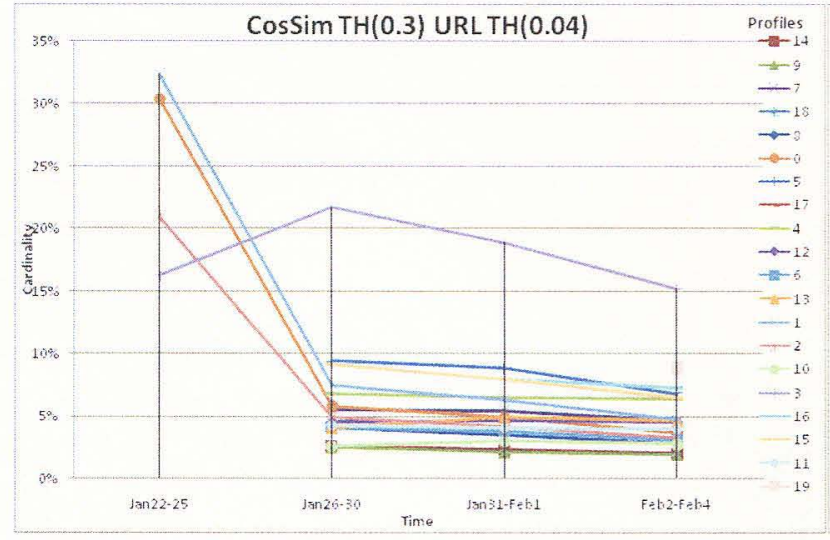

(a)

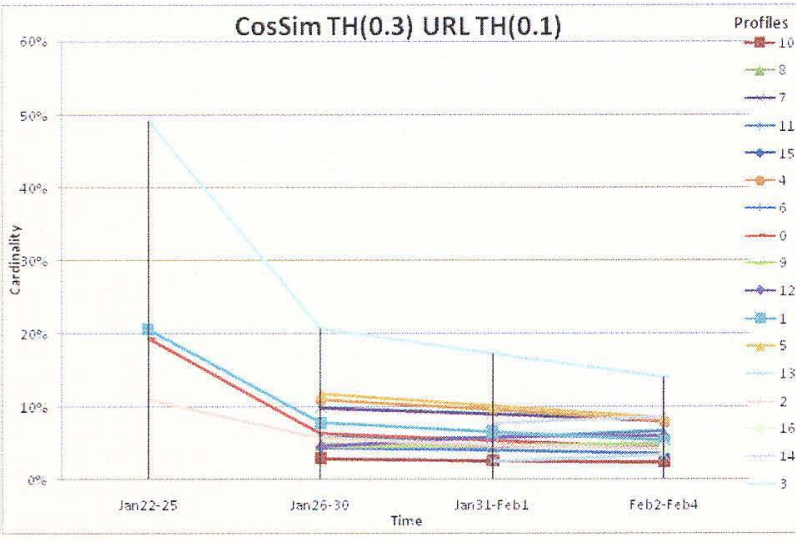

(b)

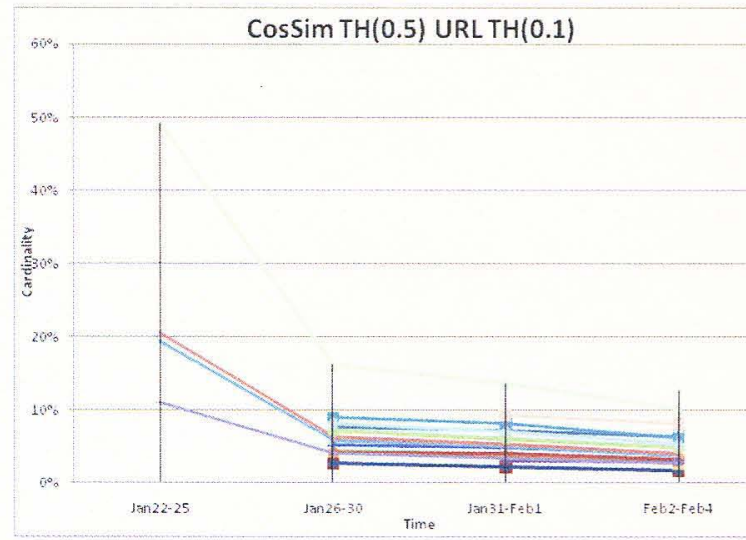

(c)
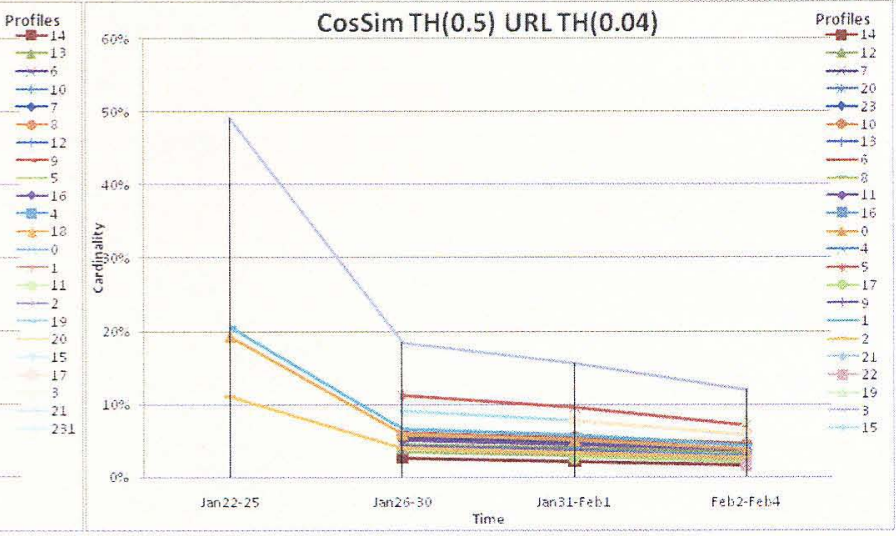

(d)

Similarity. The cardinality of the profile at each time period represents all sessions matching this profile up to (and including) that time period. The cardinality is always normalized by dividing it by the sum of all profile cardinalities up to that time. The profiles are ordered (in a decreasing order) based on their variance.

All configurations in Fig.4.12 show similar behavior where the cardinality is decreasing slightly over time. The normalized cardinality is affected by the number of profiles discovered at each time period, since a large number of profiles means more competition on new sessions, and hence lower cardinality.

Fig.4.13 shows the profile cardinalities when using the Robust Weight Similarity. 
Figure 4.13: Missouri: Evolution of Profile Cardinality Percentages (RobWT)

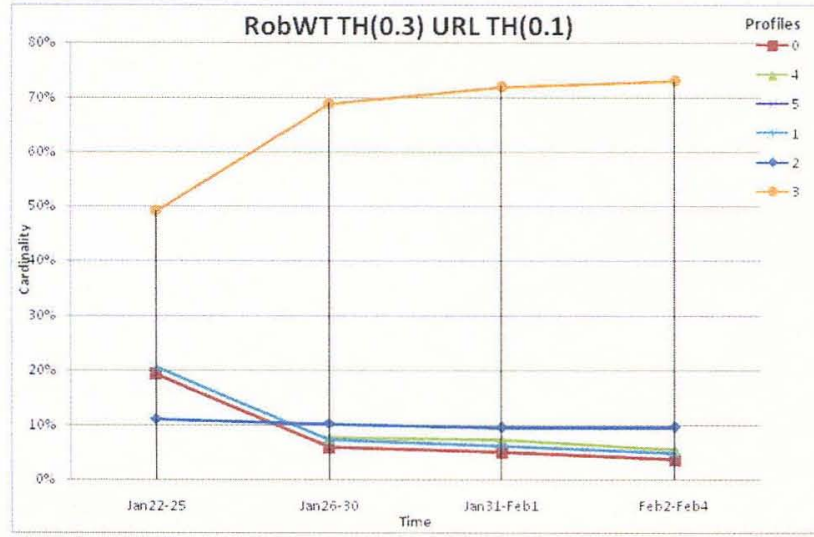

(a)

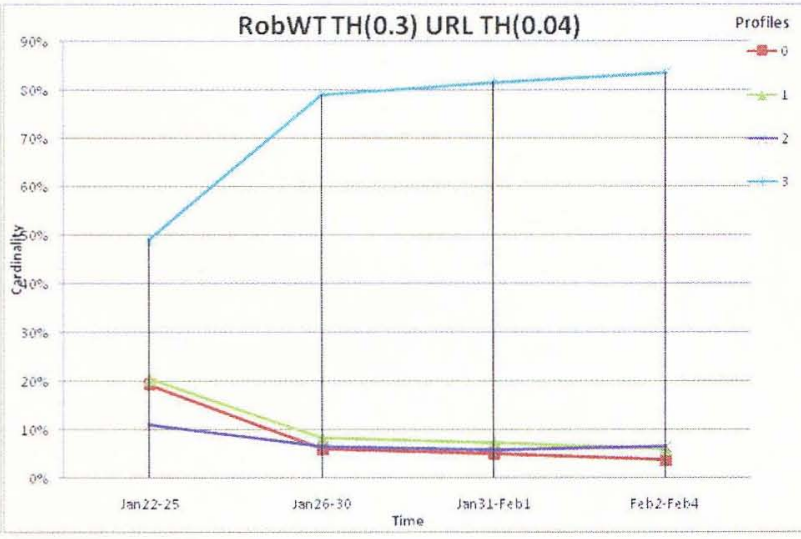

(b)

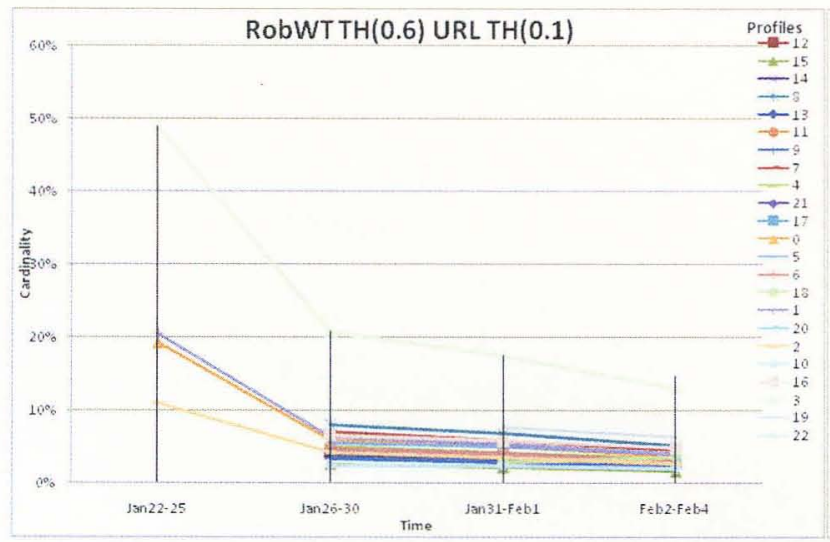

(c)

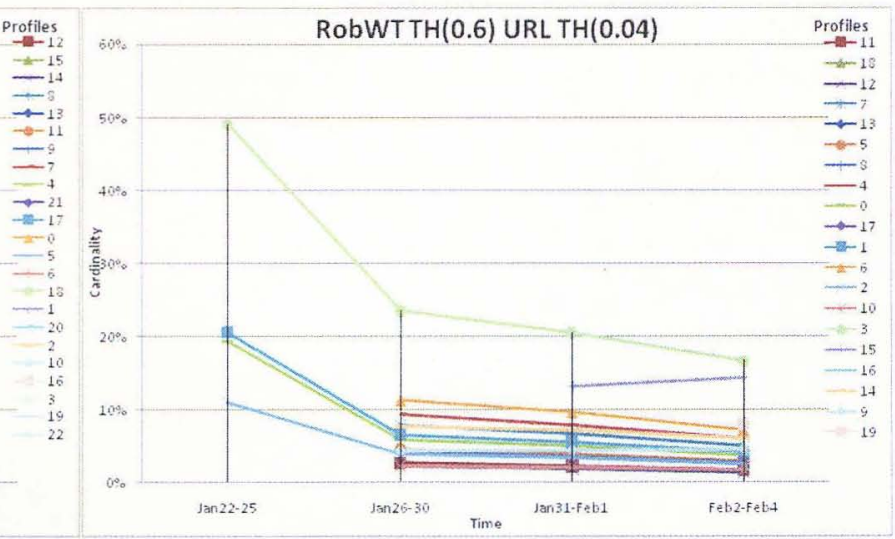

(d)

All configurations show that the majority of profiles are decreasing their normalized cardinality, except for two profiles in Fig.4.13(a) and (b). These two profiles do not have a lot of competition (because a low number of profiles were discovered), so they keep increasing their cardinality. However, both of them contain the highest variance which indicates that these profiles are of low quality, and most likely are the "default" profiles where the majority of new sessions are added.

\subsubsection{Profile Count}

The number of profiles generated at the end of evolution for each configuration is 
Figure 4.14: Missouri: Total Profiles Count

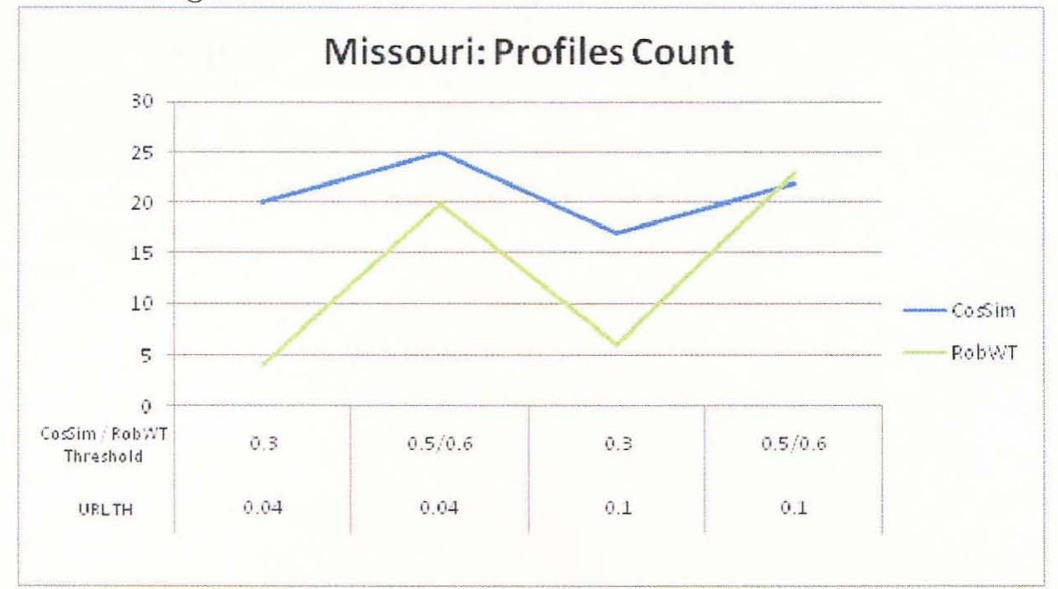

shown in Fig.4.14. A common trend between both matching methods (CosSim and RobWT) is that the number of profiles increases when a higher similarity threshold value is used. This is due to the fact that when fewer sessions succeed to match the current profiles (due to the higher matching similarity threshold), the distinct sessions would be forced to generate new (distinct) profiles. Another observation is that the URL weight threshold has minimal affect on the number of profiles for both matching methods.

\subsubsection{Profile Pair-wise Similarity}

A powerful profile discovery quality evaluation metric is to find how similar the profiles that result from the pattern discovery are to each other. If profiles are too similar to each other, then the updating algorithm was not able to match the new logs with the existing profiles accurately.

A difference matrix is created to find the pair-wise similarity between every two profiles at the end of evolution. The maximum, minimum, average, and median similarity is plotted in Fig.4.15, as well as the percentage of duplicate profiles. Duplicate profiles are defined as the ones with difference less than 0.01 . This translates to pro- 
Figure 4.15: Missouri: Profile Pair-wise Similarity Aggregates

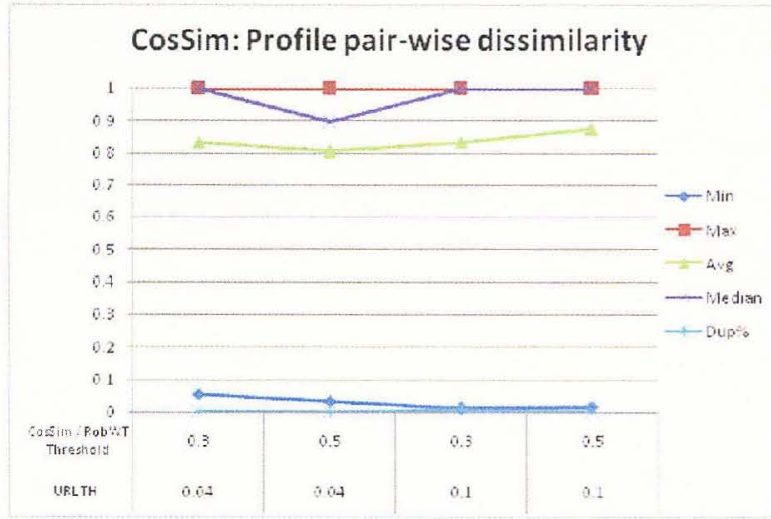

(a)

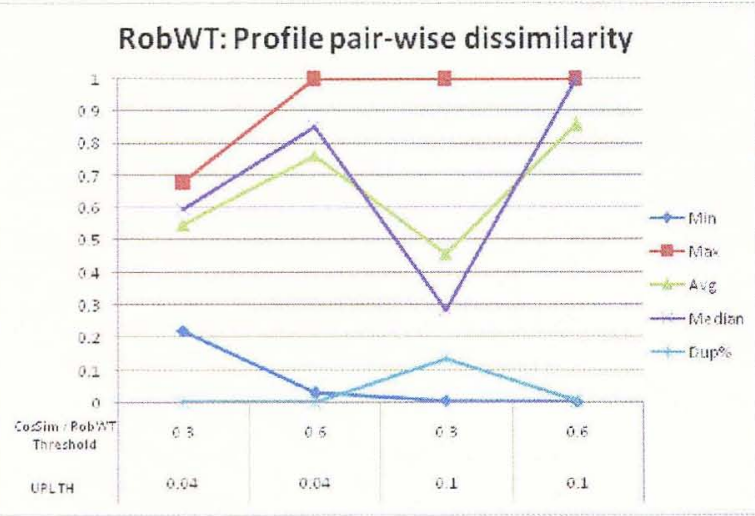

(b)

files who are at least $90 \%$ similar to each other (using binary cosine similarity), since $(1-0.9)^{2}=0.01$. See Section 4.1.1.

Fig.4.15(a) shows a desired behavior where the maximum, median, and average pairwise similarities are all greater than 0.8 which means that they are only $10 \%$ or less similar to each other. The minimum similarity aggregate is around zero which means that there are some profiles (at least one pair) that are very similar to each other. The duplicate percentage is also around zero which means that only very few number (if any) of the profiles are duplicates.

Fig.4.15(b) shows that when the similarity threshold is low (0.3) the median and average pairwise distances are low, i.e. most profiles are only $50 \%$ different, and the duplicate percentage is about $13 \%$ which is high compared to all other configurations. However, when the RobWT threshold value becomes more strict (0.6), the behavior improves and fewer profiles are similar to each other, resulting in fewer duplicates.

\subsubsection{Profile Density}

The profile density is a quality metric that combines the profile cardinality and variance. A high density is desirable since it means a high cardinality and low variance 
at the same time. The profile density is calculated at each time period by dividing the total number of sessions that belong to the profile until that time period (i.e. cardinality at $\mathrm{T} 2$ is the sum of cardinality at $\mathrm{T} 1$ and the new matching sessions in T1) by the variance of the profile at that time period. This means that a decrease in density indicate an increase in variance (since cardinality at time period T2 is always larger than or equal to $\mathrm{T} 1$ ).

Fig.4.16 shows the density for each profile at each different time period when using the Binary Cosine Similarity as the matching criterion. The profiles were ordered (in a decreasing order) based on their variance. For most configurations, the majority of profiles maintain their density over time, except for some profiles which decrease as in Fig.4.16(a) and (b). The decrease in the density means that sessions added to the profile are not very similar to old ones, and hence increasing the variance. A stable behavior like the majority in Fig.4.16(c) and (d) means that either the profile is not gaining any new sessions (out-dated) or the new sessions that are being added are not very similar to existing ones which increase the variance slightly.

Fig.4.17 shows the profile densities when using the Robust Weight Similarity. Fig.4.17(a) and (b) show similar behavior were the profile with highest variance increases its density, and the rest of the profiles maintain a stable density over time. This means that the high cardinality profile is acquiring too many sessions (and that the increase in its variance is not enough to affect its density). This is an example of an undesirable high density, because this high-cardinality and high-variance profile seems to be the "default" cluster that all non-matching sessions are assigned to. This could not have been detected by looking only at the cardinality.

Fig.4.17(c) and (d) show more stable density over time (with some profiles increasing their density). Using the high similarity threshold have caused higher quality profiles to be discovered with a balanced ratio between their variance and cardinality. 
Figure 4.16: Missouri: Evolution of Profile Densities (CosSim)

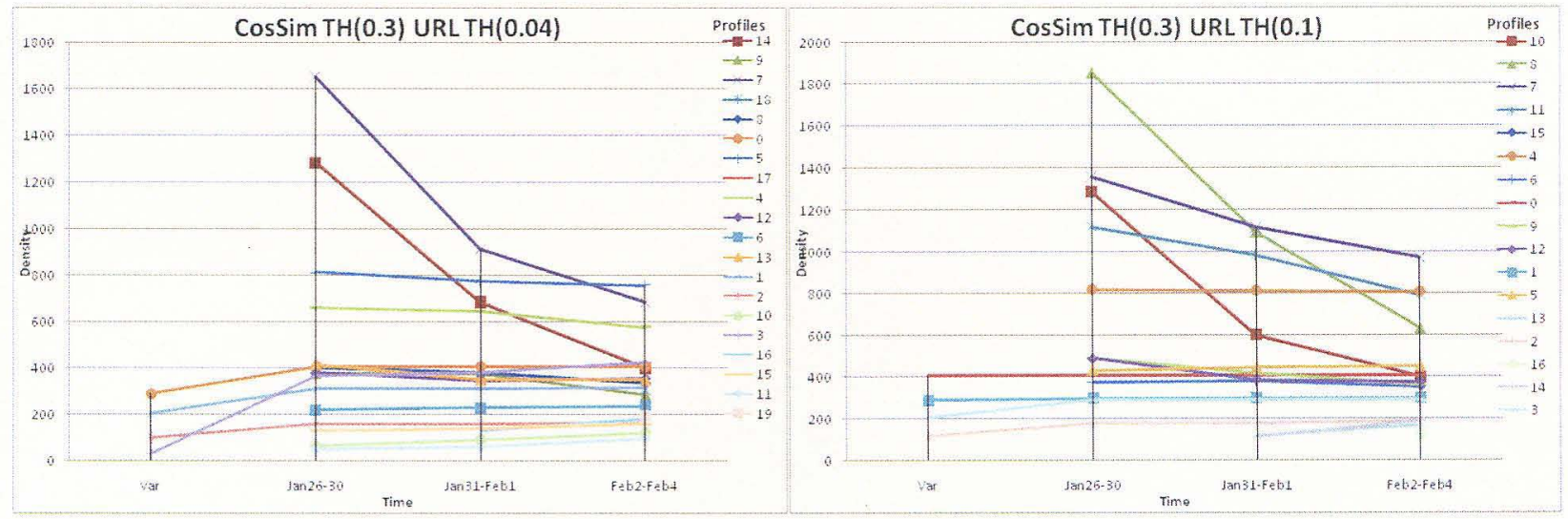

(a)

(b)

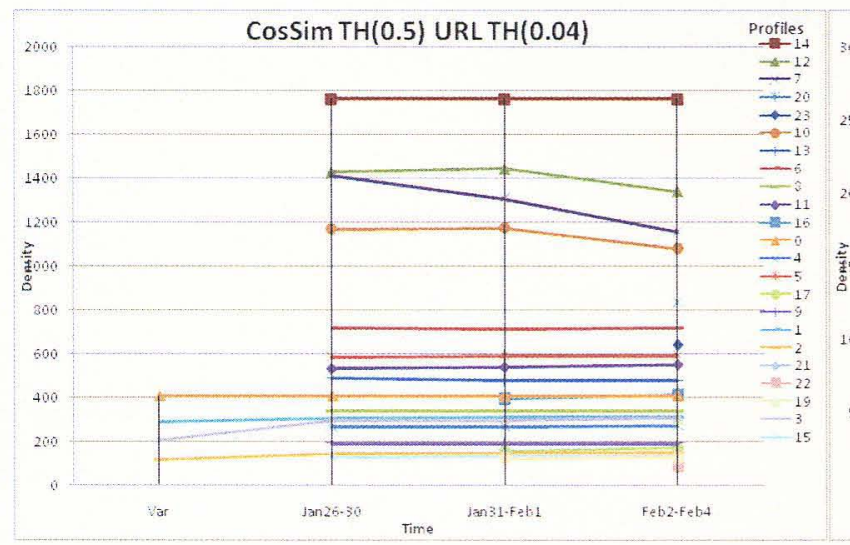

(c)

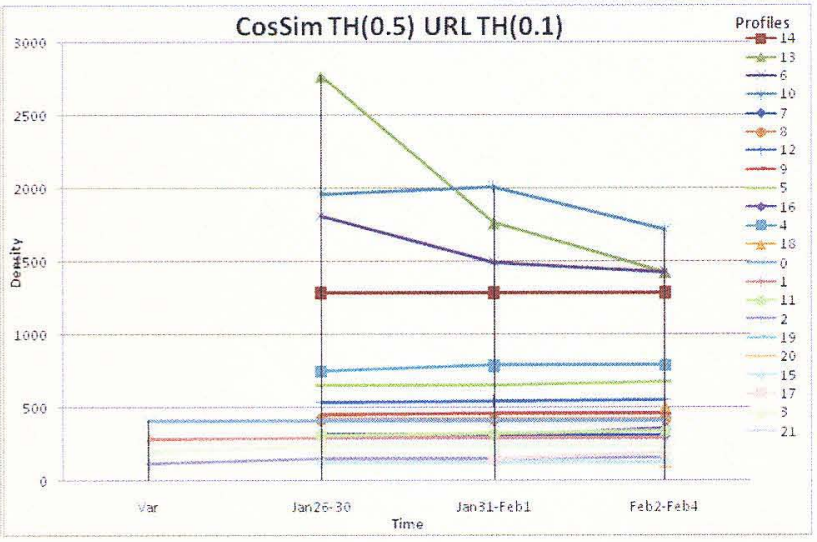

(d) 
Figure 4.17: Missouri: Evolution of Profile Densities (RobWT)

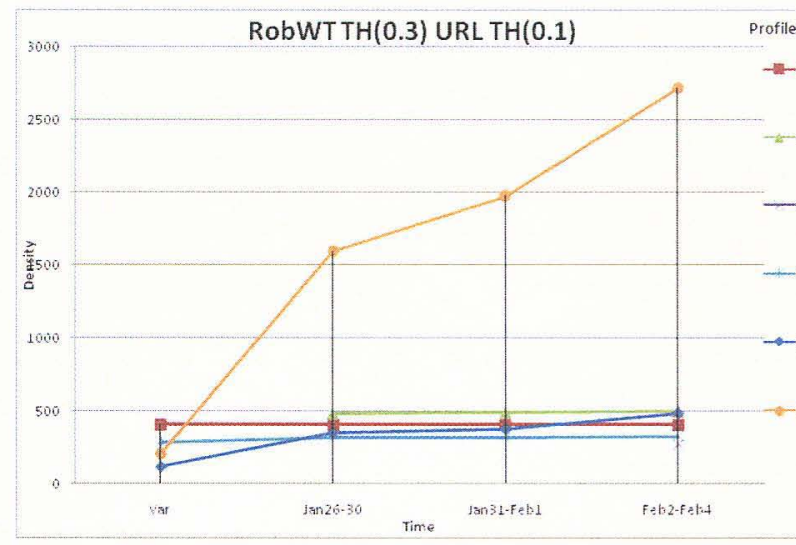

(a)
RObWT TH(0.3) URL TH(0.04)

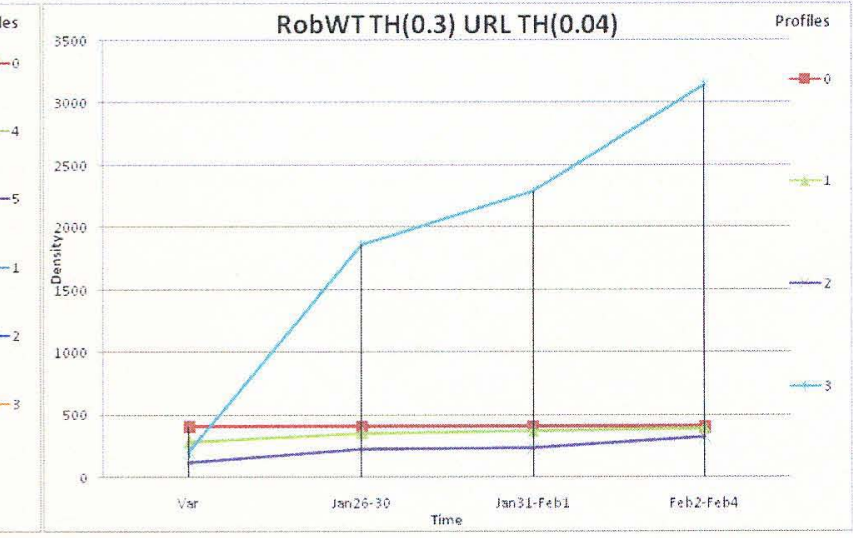

(b)

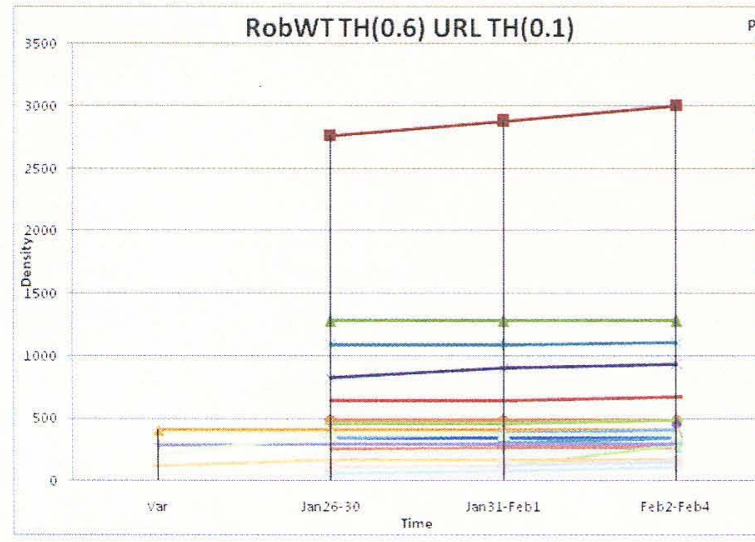

(c)

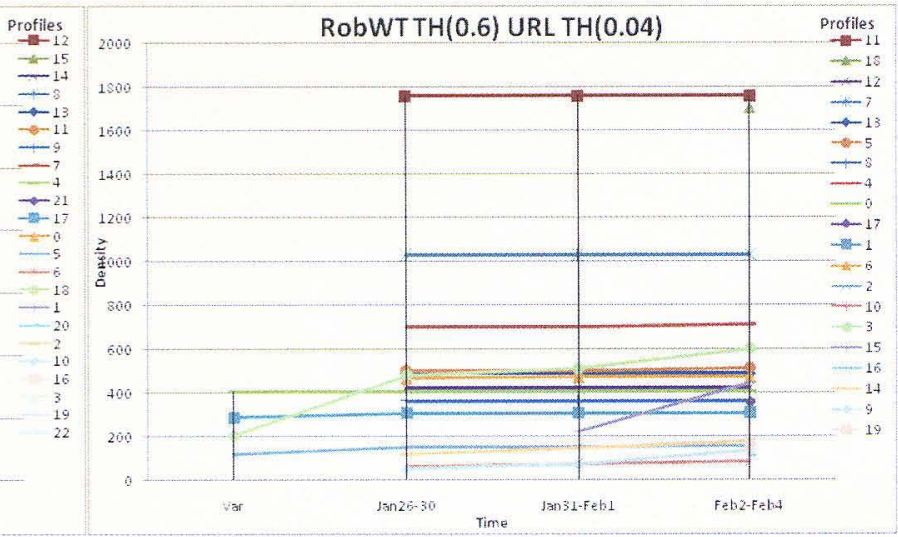

(d)

This consistent increase in densities may be the ultimate judge that matching using the Robust Weights (which depend on accurate variance estimates) yields the best quality profiles compared to Cosine Similarity matching.

\subsubsection{Evolution vs. Traditional (Full run)}

The last evaluation addresses the approach or mode of pattern discovery, which is either evolutionary (the focus of this thesis), where the pattern discovery is done in different time periods, or traditional where the full or entire usage data from all time periods is used to discover usage patterns in one shot. 
Figure 4.18: Missouri: Traditional Profile Variances

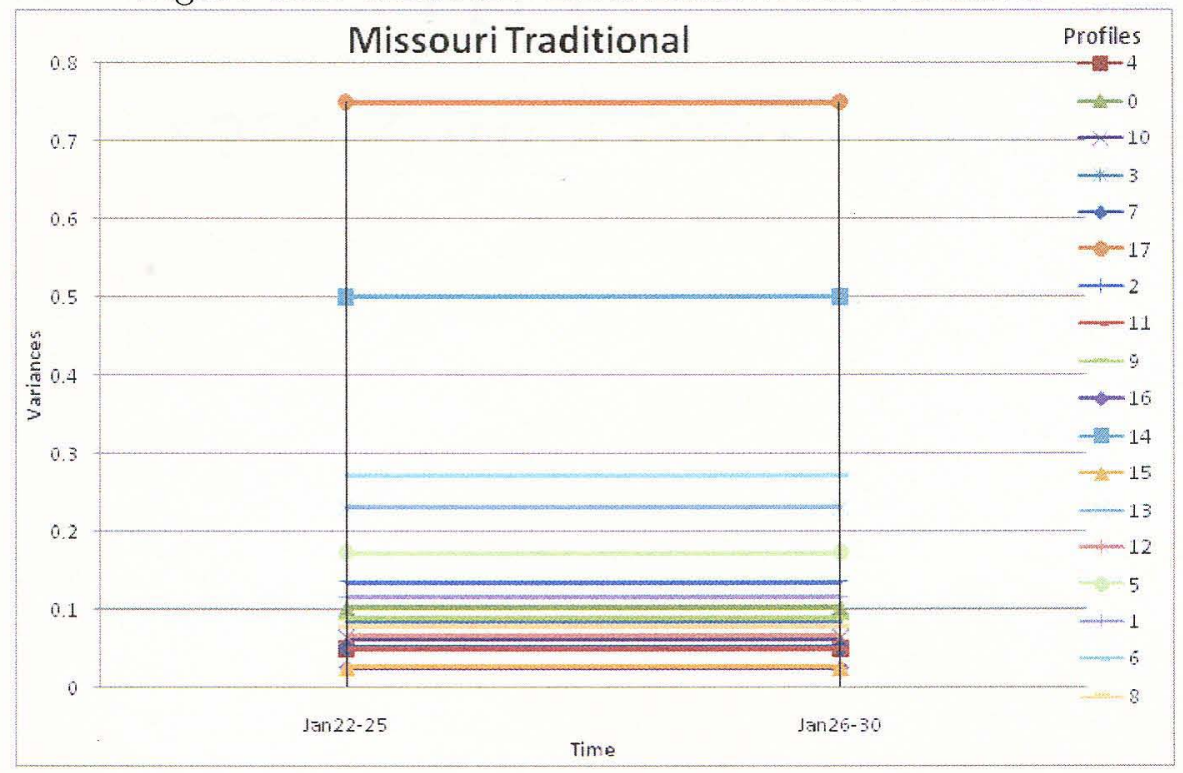

Profile variances for the traditional method are shown in Fig.4.18. The variances stay the same during all time periods (since they do not evolve), and they are shown at different times just for the purpose of illustration and for ease of comparison with Fig.4.3 and Fig.4.4 (for the evolution mode). The profiles were also ordered based on their cardinality percentage. The majority of profiles have a low variance, which means that most profiles from the traditional method are of good quality. These levels of quality were also found when using the evolutionary approach. However, we can conclude from Fig.4.4(d) that the Evolution mode was able to "refine" the profiles to lower variance (and hence improved compactness), by the end of all periods. The maximum variance was 0.6 in the evolution mode as opposed to 0.8 in the traditional mode (25\% improvement).

Fig.4.19 shows the densities of the profiles discovered when using the traditional discovery approach. The density represents the ration between the profile cardinality and its variance. Profiles are order in a decreasing order based on their cardinality percentage. All profiles are of high quality since the density is high. Some of the 
Figure 4.19: Missouri: Traditional Profile Density

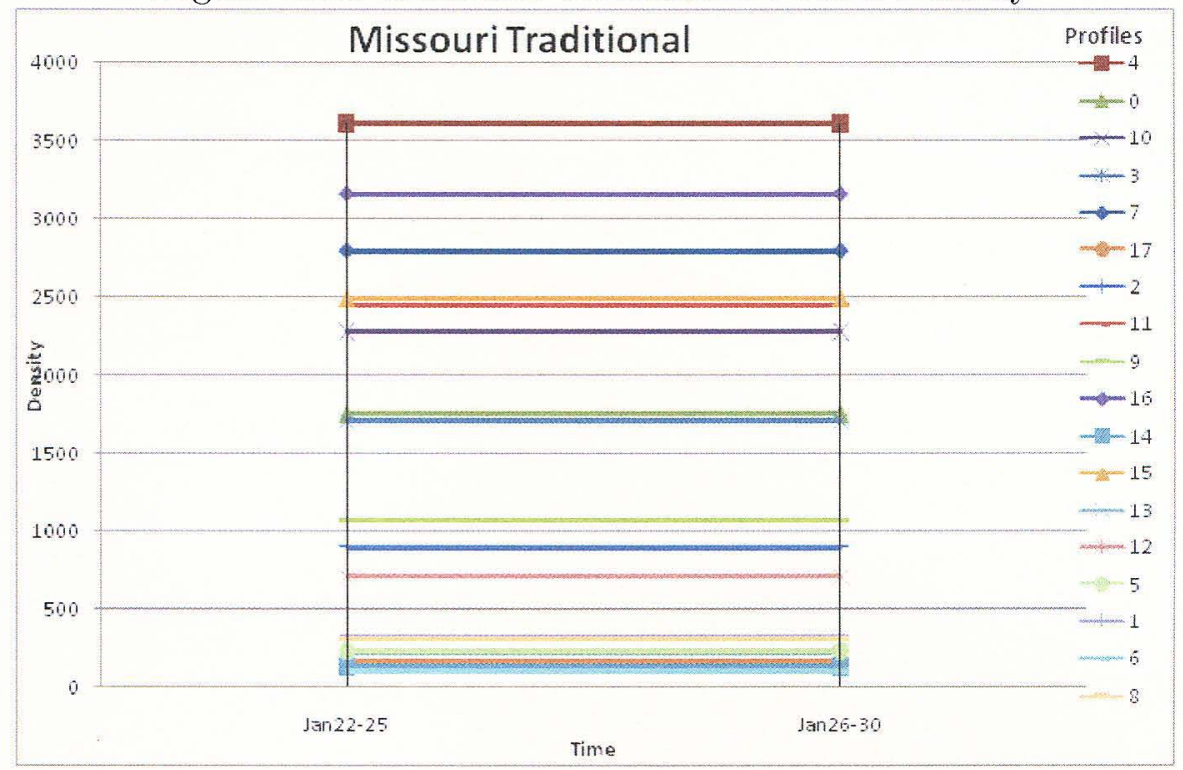

profiles generated using the evolutionary approach showed a comparable density as was seen in Fig.4.17(d).

Fig.4.20 shows the maximum, minimum, average, and median profile variance with each of the methods: Evolution (CosSim, RobWT) and Traditional (Full). The aggregate metrics for the traditional method will stay the same for different configurations, since no threshold values are used. For all aggregates, a low value is desired.

Figs.4.20(a) and (d) show that the median and average variance are always larger using the evolutionary approach (but by only 5-10\%). Fig.4.20(b) shows that the minimum variance using the evolutionary approach is larger than the traditional (full) approach when the similarity threshold is low, and is equal or less than the traditional approach when a strict similarity threshold is used. This is because a higher similarity threshold will result in higher quality profiles (i.e. lower variance). The maximum variance in Fig.4.20(c) is similar for the traditional and evolutionary approaches when using a high similarity threshold value, but is less when using Robust Weight Similarity and a low similarity threshold. 
The quality of profiles resulting from traditional mining are expected to be of higher quality since all log data is mined at once. However, the evolutionary approach has proved that it too can discover profiles that are as good (or better) than using the traditional method. This can be seen in Fig.4.20(b) where the minimum variance for the evolutionary approach was less than that of the traditional approach.

Figure 4.20: Missouri: Evolution vs. Traditional Variance Aggregates

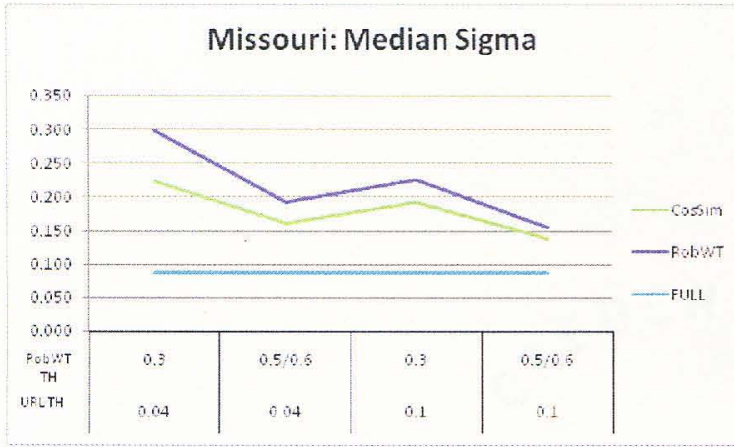

(a)

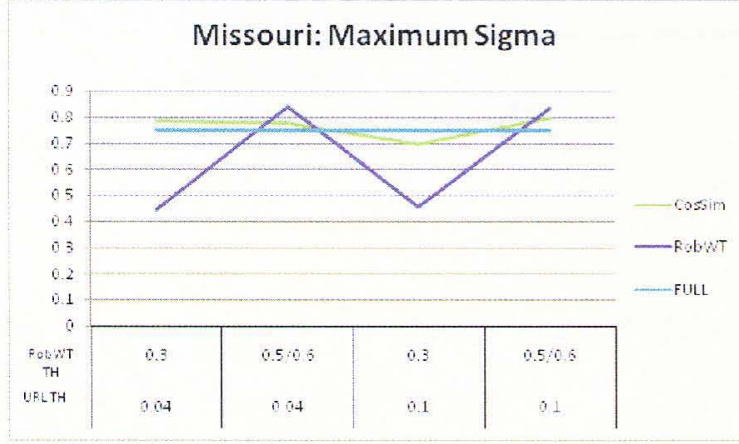

(c)

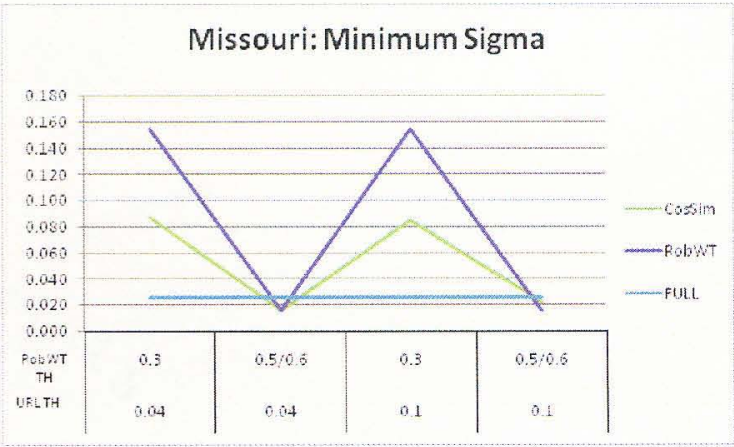

(b)

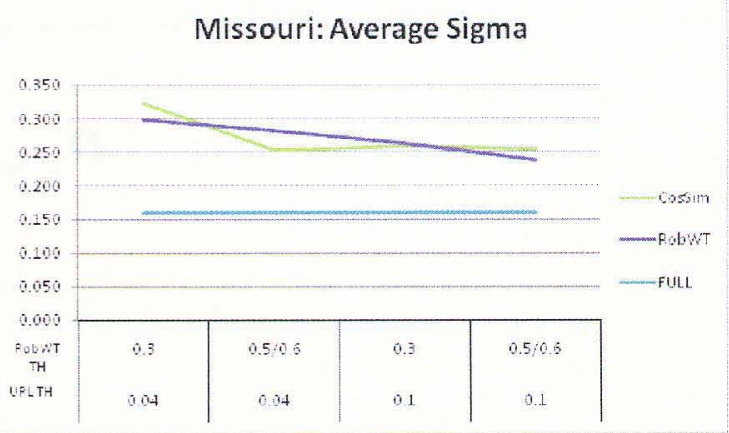

(d) 


\subsection{Experiment 2: University of Louisville Library}

\subsubsection{The Dataset}

This experiment will be conduced on a larger and much more recent dataset. The logs were collected for requests done to the University of Louisville's Library main website (library.Louisville.edu).

The logs were collected for five consecutive days: from Wednesday February 27th, 2008 till Sunday March 2nd, 2008. There were a total of 364,409 requests, grouped into 14,888 sessions. The profile evolution tracking was done on a daily basis, so there were five time periods:

- Wednesday Feb 27th 2008: 104,794 access requests (28.76\%), 4,196 sessions $(28.18 \%)$

- Thursday Feb 28th 2008: 92,446 access requests (25.37\%), 3,657 sessions (24.56\%)

- Friday Feb 29th 2008: 70,722 access requests (19.41\%), 2,919 sessions (19.61\%)

- Saturday March 1st 2008: 40,834 access requests (11.2\%), 1,791 sessions (12.03\%)

- Sunday March 2nd 2008: 55,613 access requests (15.26\%), 2,325 sessions (15.62\%)

Fig.4.21 shows the percentage of "bad access" and "good access" requests. Bad access requests include irrelevant and noise requests from search engines requests and requests that resulted in an erroneous status code. Good access requests are the remaining non-error generating requests. Just looking at the figure shows the importance of the preprocessing phase, because all these bad requests (around 85\%) would have adversely affected the resulting profiles discovery. Typically graphics requests may amount to 3-4 graphics per page or more (e.g. background picture, top banners, etc), that is why they end up being the majority of requests. 
Figure 4.21: U of L Library: Access Requests

$U$ of $L$ Library Access

Requests

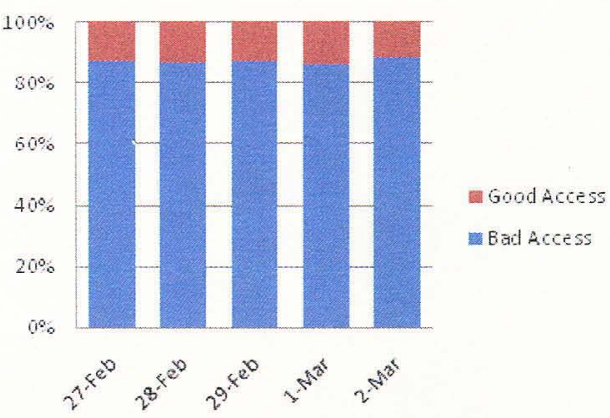

Table 4.5 shows the list of all profiles that resulted from mining this dataset at the last time period (March 2). These profiles are the result of using Robust Weight Similarity with threshold value of 0.6 , and URL threshold value of 0.1 . Only URLs with weight greater than 0.1 are shown. URLs were sorted in a descending order by the URL significance weight.

\subsubsection{Profile Variances}

The profiles evolution over time is shown in the Figures 4.22, 4.23, 4.24, and 4.25. Since the number of profiles is large for some configurations (about 50), they were separated in two graphs for the same configuration to avoid clutter: one for the highest cardinality profiles, and the other for the lower cardinality profiles. Separating profiles based on cardinality makes the comparison more accurate, because a low cardinality profile is much more sensitive to changes than a higher cardinality profile.

Fig.4.22 shows the profile variances when using a URL threshold of 0.04 and Binary Cosine Similarity for matching sessions. Figs.4.22(a) and (b) show that the majority of profiles (both high and low cardinality) start with low variance, then increase a little bit until they stabilize, which indicates good quality profiles. However, the 
Table 4.5: U of L Library: Final Profiles for RobWT (0.6) and URL TH(0.1)

\begin{tabular}{|c|c|c|c|c|}
\hline Profile & Url\# & Card & Variance & Content \\
\hline 0 & 2 & 667 & 0.0229 & /, /promo/bookplates.swf, \\
\hline 1 & 78 & 1747 & 0.291953 & /kornhauser, \\
\hline 2 & 5 & 157 & 0.088875 & /research/sub/dbsA.html, /research/sub/dbsDE.html, /research/sub/dbsP.html, \\
\hline 3 & 5 & 724 & 0.100414 & /,/research/sub/dbsA.html, /promo/bookplates.swf, \\
\hline 4 & 7 & 102 & 0.056861 & /research/sub/dbsA.html, /research/sub/dbsJKL.html, /,/promo/bookplates.swf, /research/sub/dbsDE.html, \\
\hline 5 & 18 & 61 & 0.186887 & $\begin{array}{l}\text { /research/sub/dbsA.html, /top/subjects.html, /,/promo/bookplates.swf, /research/sub/dbsDE.html, /research/sub/dbsP.html, } \\
\text { /research/sub/dbsJKL.html, /research/business/index.html, /top/tools.html, /research/panafrican/index. html, /research/health/index.html, } \\
\text { /research/sub/dbsNO.html, }\end{array}$ \\
\hline 6 & 6 & 45 & 0.059365 & /research/sub/dbsA.html,/research/sub/dbsM.html, /,/promo/bookplates.swf, \\
\hline 7 & 1 & 386 & 0.005911 & /music, \\
\hline 8 & 2 & 100 & 0.00968 & /music/listenonline.html, /music, \\
\hline 9 & 6 & 77 & 0.056598 & l, /music, /promo/bookplates.swf, /music/listenonline.html, \\
\hline 10 & 2 & 264 & 0.110867 & /government/news/otherlinks/otherlinks.html, /, \\
\hline 11 & 4 & 56 & 0.112673 & /ekstrom, /research/sub/dbsA.html, \\
\hline 12 & 3 & 65 & 0.055307 & /art, /promo/bookplates.swf, \\
\hline 13 & 1 & 159 & 0.007119 & /forms/z.htm, \\
\hline 14 & 1 & 154 & 0.004239 & /ekstrom/special/moi/moi-people01.html, \\
\hline 15 & 9 & 96 & 0.224964 & $\begin{array}{l}\text { /top/subjects.html, /,/research/communication/index.html, /research/anthropology/index.html, /promo/bookplates.swf, } \\
\text { /research/general/index.html, }\end{array}$ \\
\hline 16 & 2 & 64 & 0.139356 & /government/periodicals/periodall.html, \\
\hline 17 & 3 & 1166 & 0.071876 & I./promo/bookplates.swf, \\
\hline 18 & 5 & 83 & 0.02923 & /research/sub/dbsA.html, /research/sub/dbsP.html, /,/promo/bookplates.swf, \\
\hline 19 & 5 & 36 & 0.058454 & /research/sub/dbsJKL.html, /research/sub/dbsA.html, /, /ekstrom, \\
\hline 20 & 8 & 78 & 0.094318 & /research/sub/dbsA.html, /research/sub/dbsDE.html, /,/promo/bookplates.swf, /research/sub/dbs.JKL.html, \\
\hline 21 & 8 & 41 & 0.108382 & /research/sub/dbsWXYZ.html, /research/sub/dbsA.html, /,/promo/bookplates.swf, /top/subjects.html, \\
\hline 22 & 10 & 49 & 0.108915 & $\begin{array}{l}\text { /research/sub/dbsA.html, /research/sub/dbsS.html, /,/promo/bookplates.swf, /research/sub/dbsWXYZ.html, /top/subjects.html, } \\
\text { /research/sub/dbsDE.html, }\end{array}$ \\
\hline 23 & 11 & 90 & 0.252119 & /research/sub/dbsA.html, /research/sub/dbsNO.html,/,/research/sub/dbsB.html, /top/subjects.html, \\
\hline 24 & 3 & 99 & 0.052191 & fill, $/$, \\
\hline 25 & 2 & 48 & 0.142716 & /government/goodsources/factbook.html, \\
\hline 26 & 2 & 49 & 0.03262 & /art, / \\
\hline 27 & 8 & 45 & 0.180277 & /ekstrom,/,/ekstrom/hours/index.html, /job, /services/index.html, \\
\hline 28 & 4 & 105 & 0.322663 & /government/news/otherlinks/otherlinks.html, \\
\hline 29 & 2 & 450 & 0.0233 & /promo/bookplates.swf, $/$ \\
\hline 30 & 6 & 62 & 0.094646 & /research/sub/dbsA.html, /research/sub/dbsS.html, /research/sub/dbsWXYZ.html, /research/sub/dbsC.html, \\
\hline 31 & 4 & 445 & 0.120767 & l,/research/sub/dbsA.html, \\
\hline 32 & 5 & 39 & 0.054863 & /research/sub/dbsA.html, /research/sub/dbsDE.html, /, \\
\hline 33 & 4 & 41 & 0.043474 & /research/sub/dbsA.html, /research/sub/dbsNO.html, /,/research/sub/dbsDE.html, \\
\hline 34 & 4 & 50 & 0.0783 & /research/sub/dbsA.html, /research/sub/dbsP.html, /research/sub/dbsJKL.htmi, /top/subjects.html, \\
\hline 35 & 1 & 58 & 0.024124 & /ill, \\
\hline 36 & 1 & 35 & 0.011143 & lart, \\
\hline 37 & 6 & 33 & 0.129325 & /dlls, /dils/database, /,/dlls/guide, /dlls/forms, \\
\hline 38 & 3 & 549 & 0.080418 & /,/promo/bookplates.swf, \\
\hline 39 & 7 & 124 & 0.140677 & /research/sub/dbsA.html, /,/research/sub/dbsP.html, /research/sub/dbsJKL.html, /research/sub/dbsDE.html, /research/sub/dbsNO.html, \\
\hline 40 & 8 & 41 & 0.140021 & /ekstrom, /./promo/bookplates.swf, /ekstrom/hours/index.html, /kornhauser, \\
\hline 41 & 1 & 41 & 0.01061 & /kornhauser/forms/webform.html, \\
\hline 42 & 2 & 705 & 0.085 & /,/promo/bookplates.swf, \\
\hline 43 & 4 & 48 & 0.2716 & /ekstrom, /top/subjects.html, /ekstrom/hours/index.html, \\
\hline 44 & 9 & 182 & 0.34 & $\begin{array}{l}\text { /research/sub/dbsA.html, /top/subjects.html, /research/sub/dbsDE.html, /research/business/index.html, /,/research/sub/dbsP.html, } \\
\text { /research/sub/dbsJKL.html, /promo/bookplates.swf, }\end{array}$ \\
\hline 45 & 2 & 29 & 0.0873 & /government/states/kentucky/kylit/berry.html, /government/states/kentucky/kylit/berryadd.html, \\
\hline 46 & 5 & 30 & 0.4754 & /government/news/otherlinks/otherlinks.html,/government/subjects/health/daterape.html, \\
\hline
\end{tabular}


top three profiles $(17,18$, and 26$)$ have the highest cardinality and a relatively high variance, and they don't seem to improve over time, i.e. their variance stays almost the same during evolution.

When using a stricter similarity threshold in Figs.4.22(c) and (d), the quality of profiles seems to improve. The majority of profiles start with and keep a stable low variance over time, and the high cardinality profiles even seem to improve their variances, e.g. profile number 1 . Therefore, an initial conclusion from these results is that a stricter similarity threshold resulted in better quality profiles, since only close sessions are used to update profiles.

Fig.4.23 shows the profiles evolution for a higher URL threshold (0.1) and using Binary Cosine Similarity. Figs.4.23(a) and (b) shows that all profiles are of high quality with variance less than 0.5 . However, some of them have a tendency to increase their variance. Figs.4.23(c) and (d) (with strict matching) show more stable variance values with the majority under 0.3 , with the exception of the profile with highest cardinality (profile 1) which starts with high variance and later improves its variance. The results shown in this figure point to a more desirable behavior than the ones in Fig.4.22. Hence, a higher URL threshold caused more stability and better quality of profiles.

For the Robust Weight Similarity, Fig.4.24 shows the variance evolution when using 0.04 as the URL weight threshold value. For 0.3 similarity threshold, fewer profiles are developed and their profile variances are shown in Fig.4.24(a), which shows that profiles starting with low cardinality have increased their variances slightly, while profiles with higher cardinality started with high variance and then decreased their variances, with most profiles finally stabilizing around an average variance of 0.5 . 
Figure 4.22: U of L Library: Evolution of Profile Variances (CosSim, URL TH(0.04))

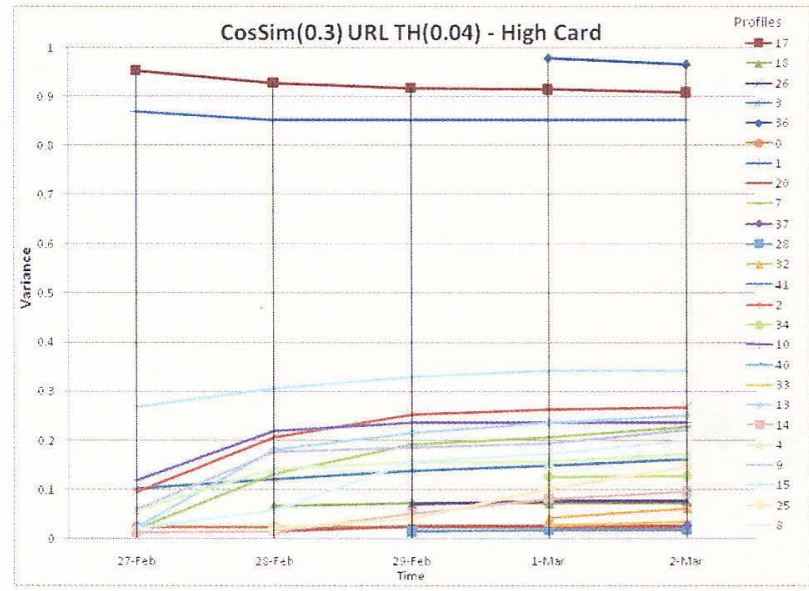

(a)

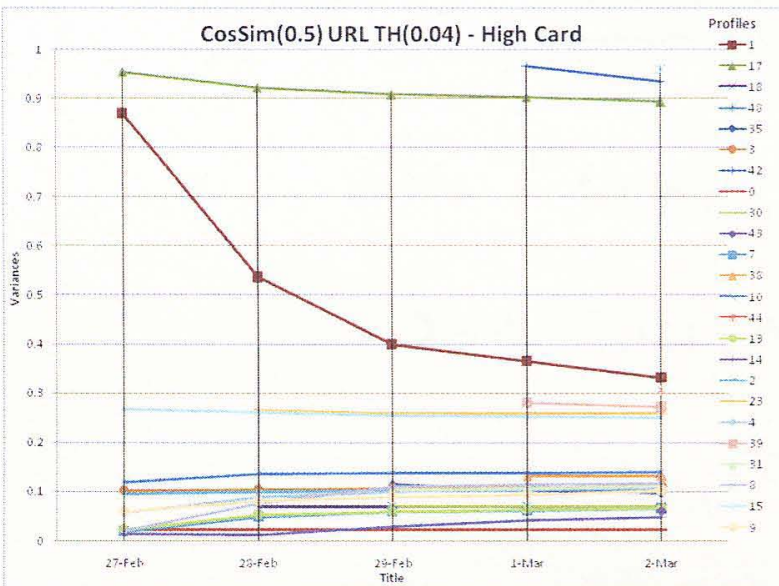

(c)

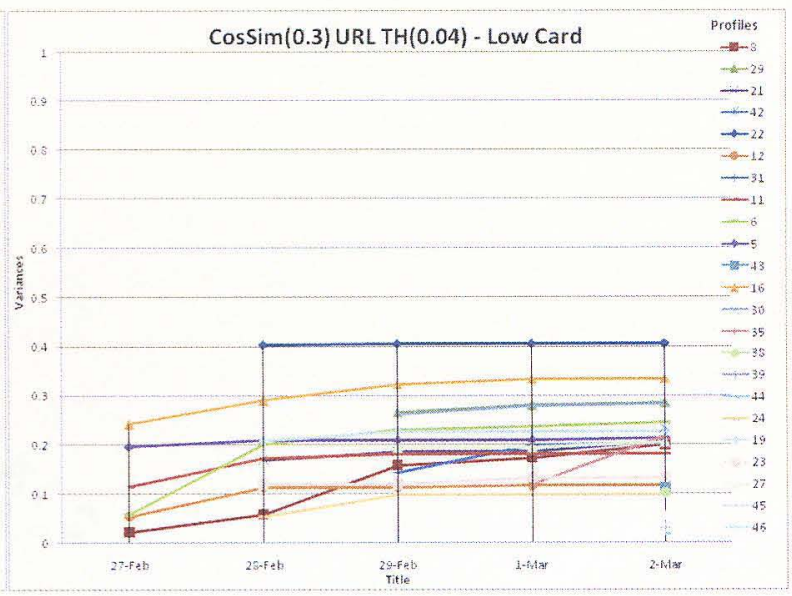

(b)

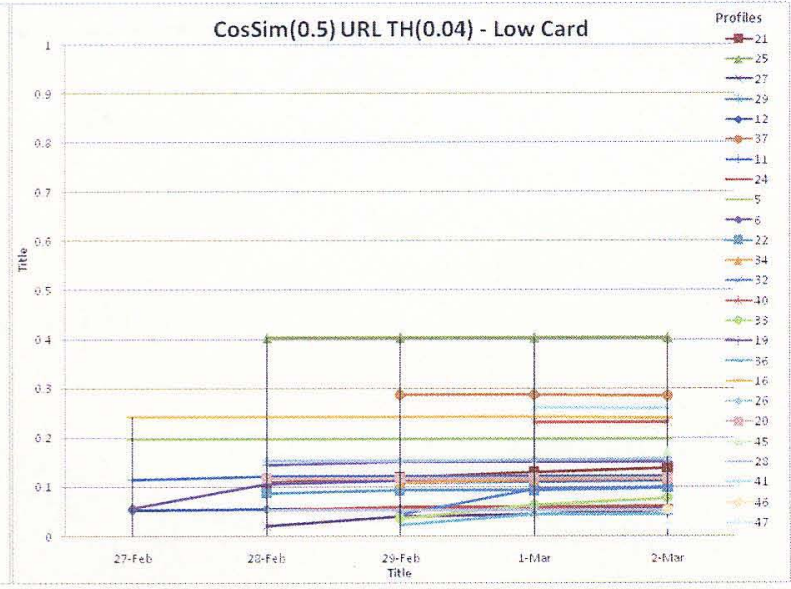

(d) 
Figure 4.23: U of L Library: Evolution of Profile Variances (CosSim, URL TH(0.1))

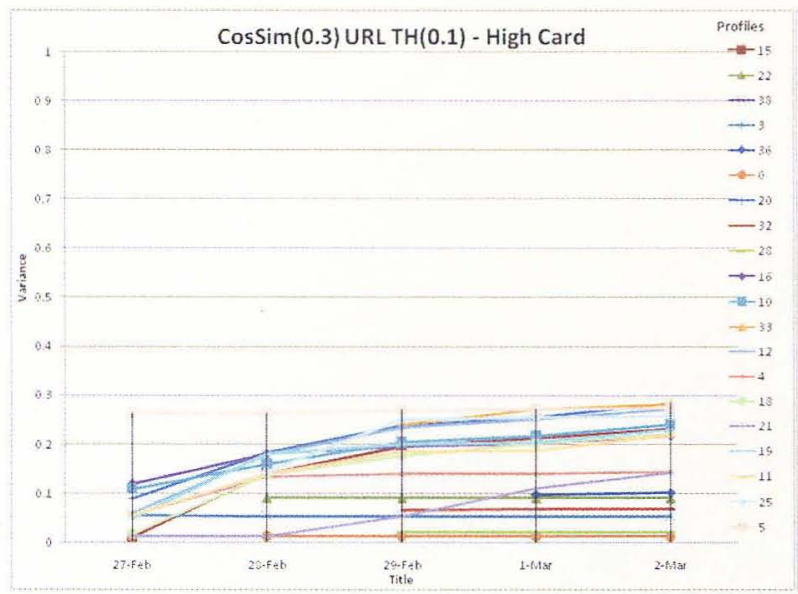

(a)

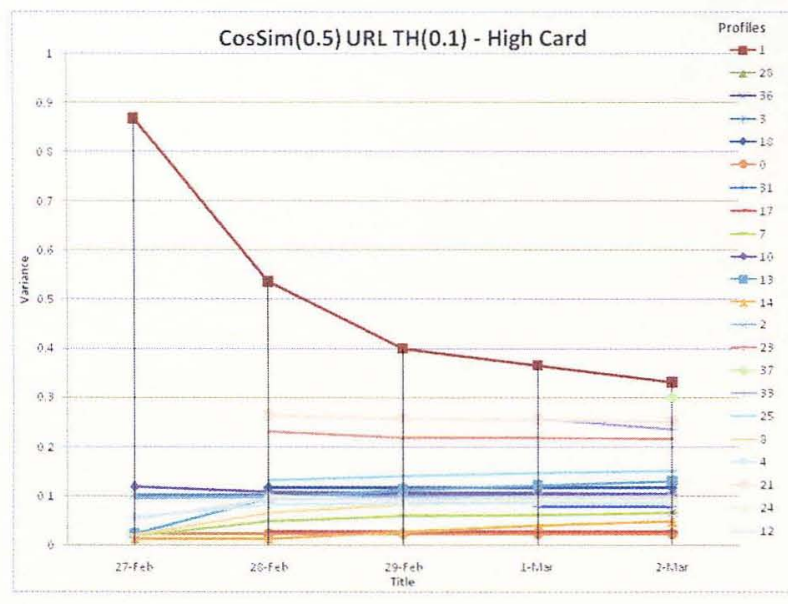

(c)

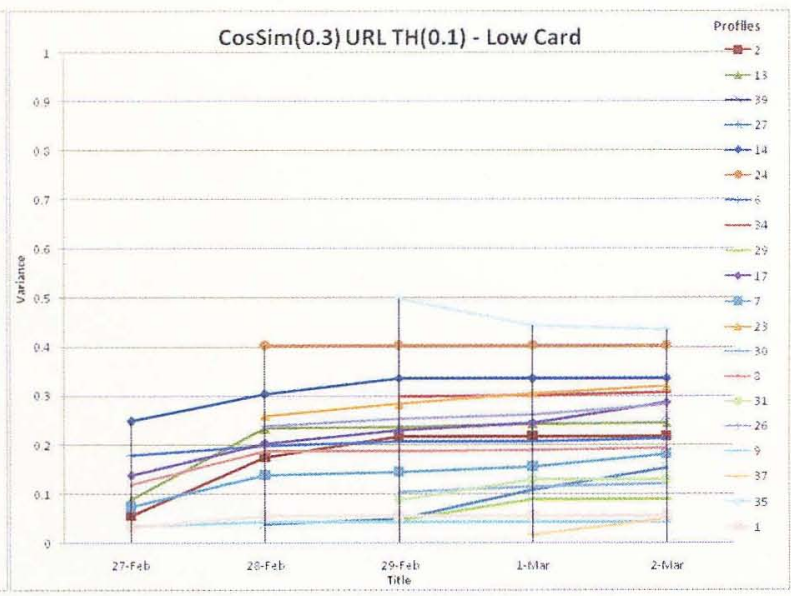

(b)

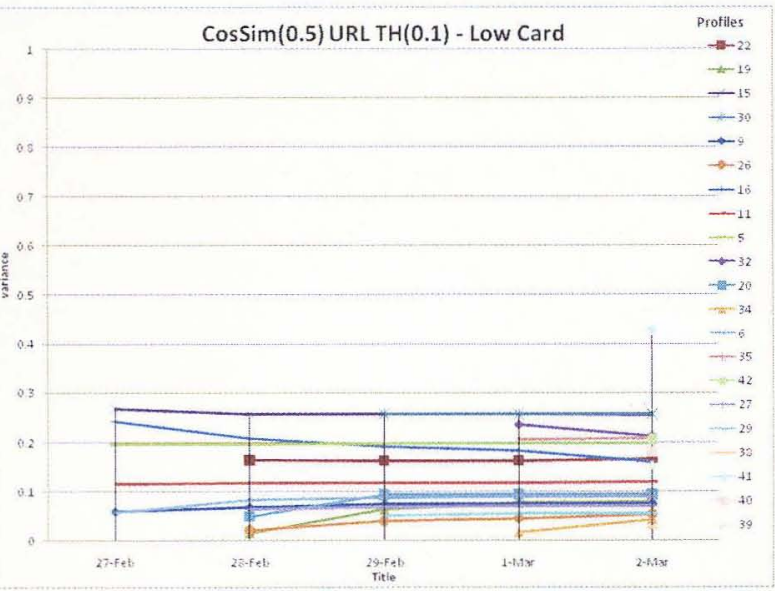

(d) 
Figs.4.24(b) and (c) show that more profiles were discovered with a higher matching threshold. The majority of profiles have stable variance changes, except the highest cardinality profiles which start with high variance that later start decreasing slightly. As in Fig.4.22, we can conclude that a stricter matching threshold has resulted in higher quality and more detailed profiles. Comparing the results from this configuration to Figs.4.22(c) and (d), we can see that both seem to show similar behavior. Thus, using the two methods of similarity (CosSim and RobWT) with a more restricted threshold value can be expected to cause the majority of profiles to have low and stable variance evolution over time.

Fig.4.25 shows the variances when using a more restrictive URL threshold value of 0.1. Fig.4.25(a) shows a similar behavior to Fig.4.24(a), where a low number of profiles were discovered with the majority of profiles having low variances, that increased slightly with the exception of the highest cardinality profile, for which the variance decreased from a high value to an average value.

The last configurations in Figs.4.25(b) and (c) show the best quality of profiles, because most profiles began with a small variance and they kept improving, in contrast with the other configurations where the variances either remained the same or increased. Even the high cardinality profiles which started with high variance, drastically decreased in their variance with time to a low value.

An overall conclusion can be drawn that more restrictive threshold values combined with the use of the profile-sensitive Robust Weight Similarity tend to result in very high quality profiles over time.

To study the overall evolution behavior profile quality, Fig.4.26 shows the minimum, maximum, median, and average variance of the final profiles at the end of 
Figure 4.24: U of L Library: Evolution of Profile Variances (RobWT , URL TH(0.04))

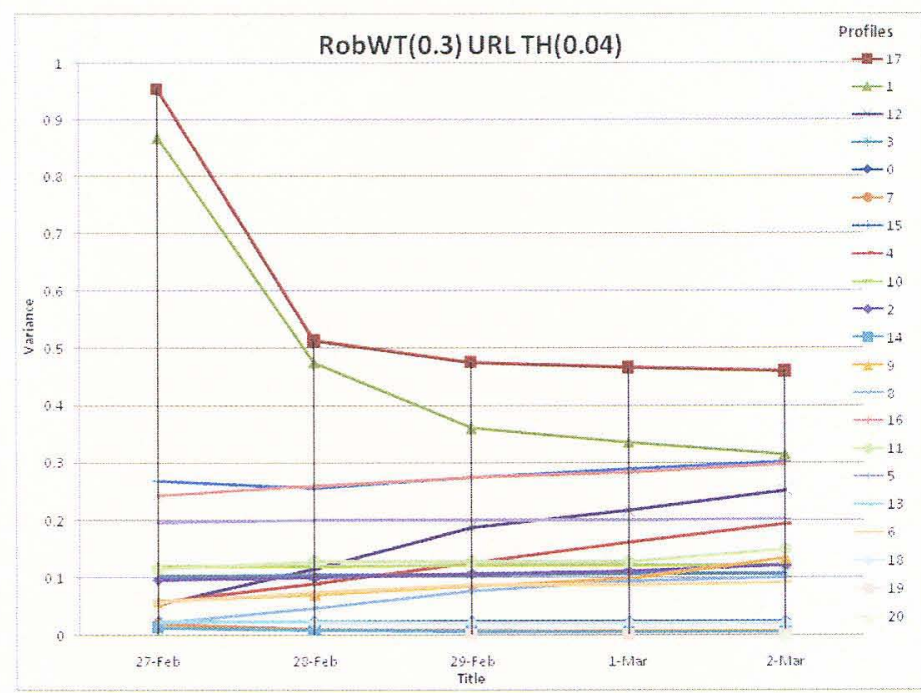

(a)

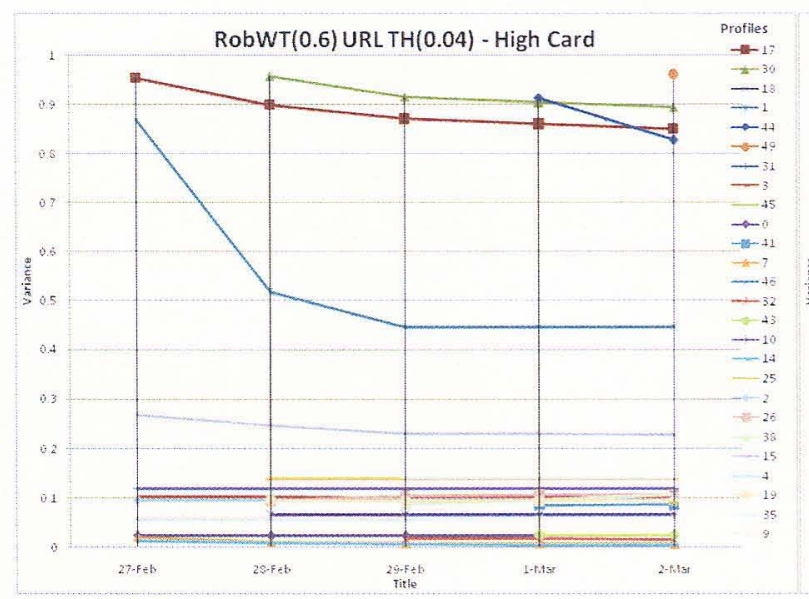

(b)

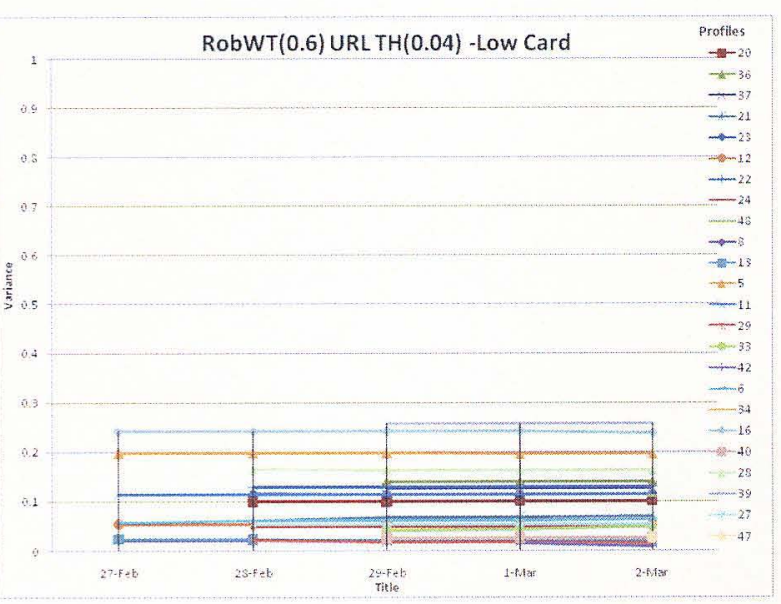

(c) 
Figure 4.25: U of L Library: Evolution of Profile Variances (RobWT, URL TH(0.1))

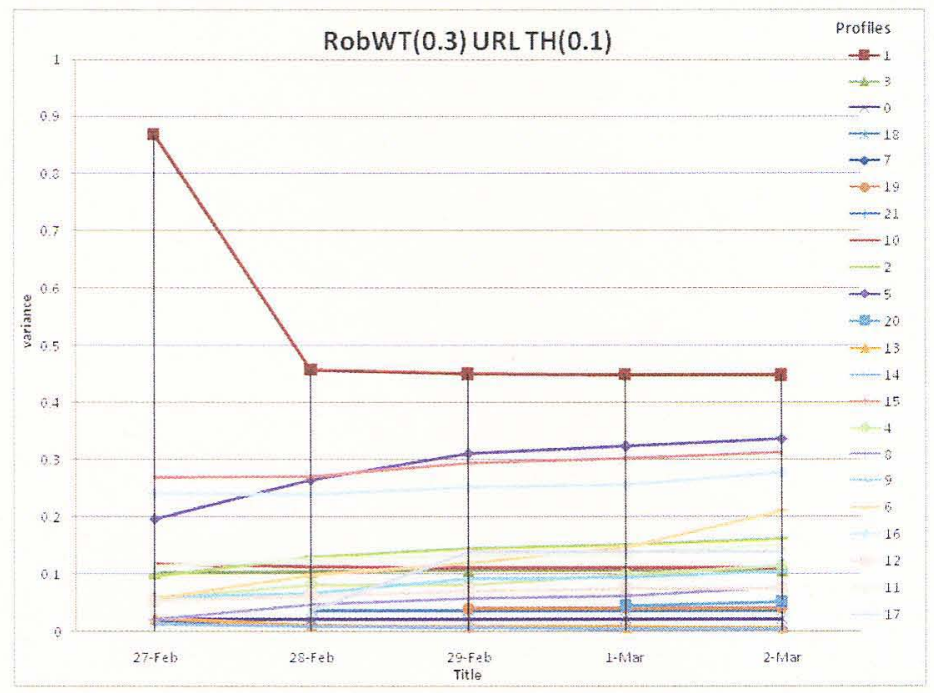

(a)

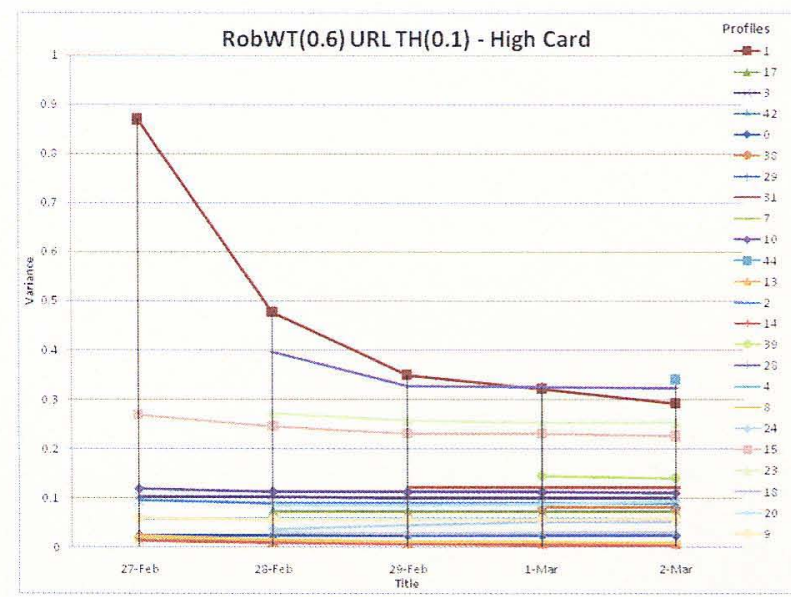

(b)

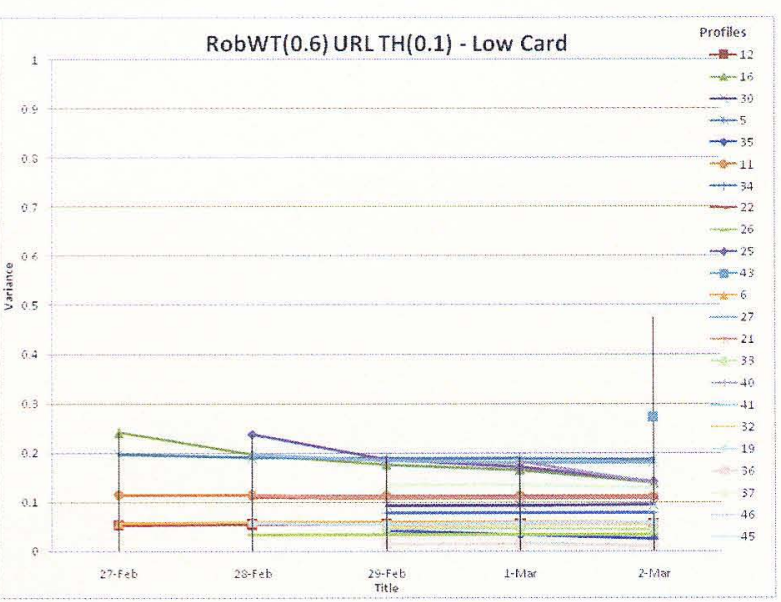

(c) 
evolution. As was noticed in Fig.4.5 for the smaller Missouri dataset, the minimum, median, and average variance remained stable and within the same range for all different configurations. The median and average variances indicate that the overall quality of profiles is good, since their values are low $(<0.2)$. However, this result should be taken in context with the final number of profiles which will be discussed next.

Fig.4.26(a) shows that moving the URL weight threshold value from 0.04 to a more restrictive value of 0.1 caused the maximum variance to drop drastically. On the other hand, the value of the cosine similarity threshold did not seem to have any effect. The decrease in variance can be explained because the binary cosine similarity is computed based on the number of common URLs between a profile and a session. So a larger number of URLs (due to a lower URL threshold) will help increase this similarity and hence increase the chance of sessions passing the thresholding test. Since URL weight threshold is applied in both post-processing and updating phases, all profiles would have either more or less URLs. In case of using a low value for URL weight threshold, the number of URLs in the profile is more than when using a 0.1 threshold value. Therefore more sessions will pass the thresholding test, and lower quality profiles are thus generated. This is why the maximum variance value is more sensitive to the URL weight threshold.

Fig.4.26(b) shows an opposite behavior, where the similarity threshold has more effect on the value of the maximum variance. In contrast to the Binary Cosine Similarity, the Robust Weight Similarity takes into account the profile variance, not just the number of URLs in the profile. Hence, if the profile has low variance, it becomes harder for new sessions to match the profile. For this reason, changing the number of URLs in the profile by changing the URL threshold value will not have the same affect as it had when using the Binary Cosine Similarity. 
Figure 4.26: U of L Library: Profiles Sigma Aggregates

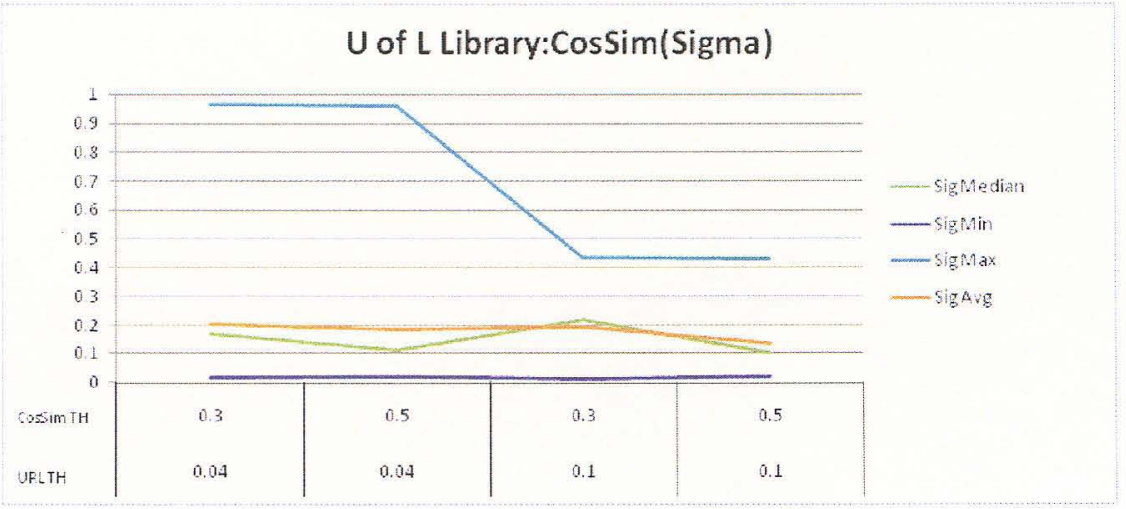

(a)

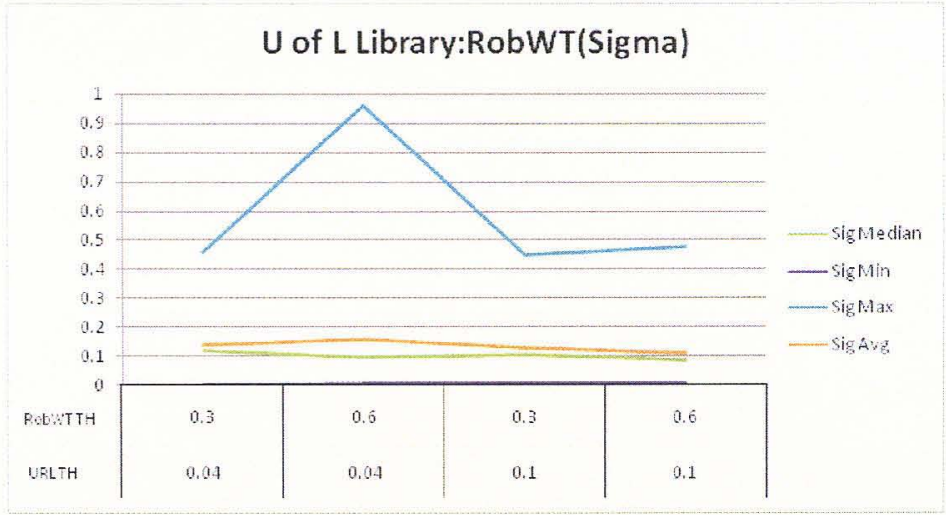

(b) 
Figure 4.27: U of L Library: Profile Counts (Binary Cosine Similarity)

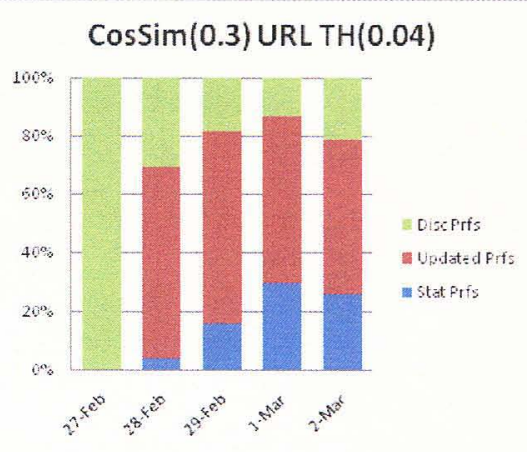

(a)
$\operatorname{Cos} \operatorname{Sim}(0.5)$ URL TH(0.04)

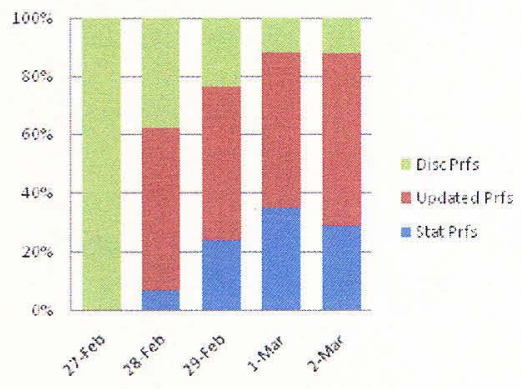

(b)

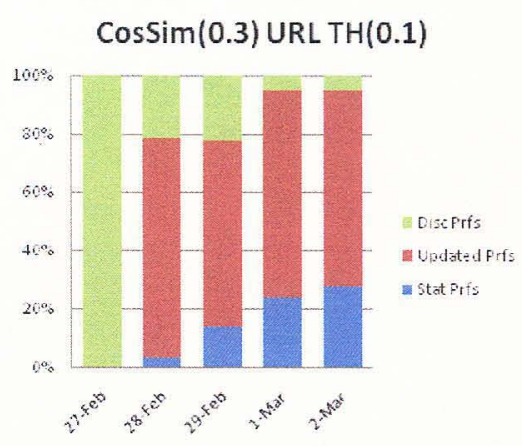

(c)

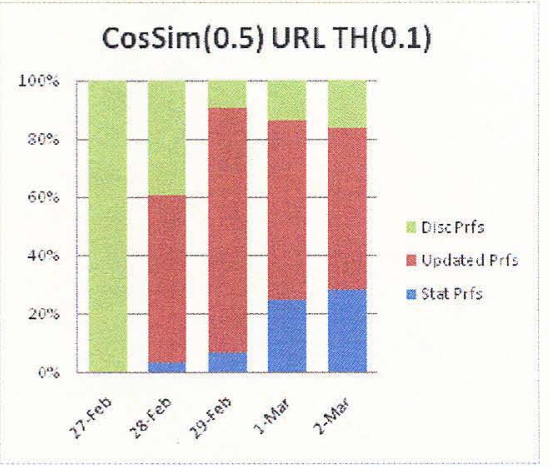

(d)

\subsubsection{Profile Evolution}

The percentage of each type of profiles discovered/updated for each time period when using the Binary Cosine Similarity is shown in Fig.4.27. The percentages of profiles seem to be similar using the different configurations, with the exception of Fig.4.27(c), where more profiles got updated on Feb 28th (Day 2), and fewer distinct profiles where discovered on March 2nd (Day 5). In all four configurations, the majority of profiles got updated, while a few profiles were discovered.

Figure 4.28 shows the profile percentages when using the Robust Weight Similarity for matching. Figs.4.28(a) and (c) show similar behaviors, where the vast majority of profiles got updated during all time periods. This can be explained for the low 
Figure 4.28: U of L Library: Profile Counts (Robust Weight Similarity)

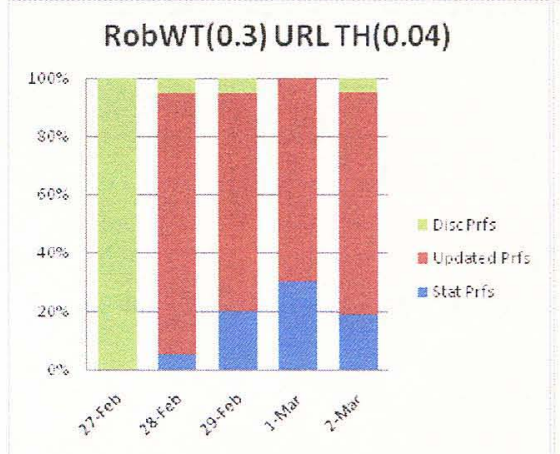

(a)

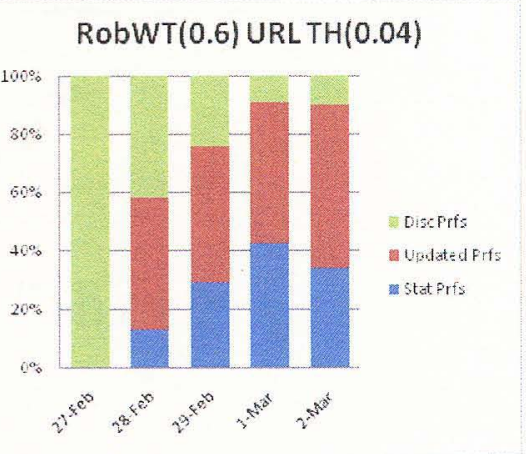

(b)

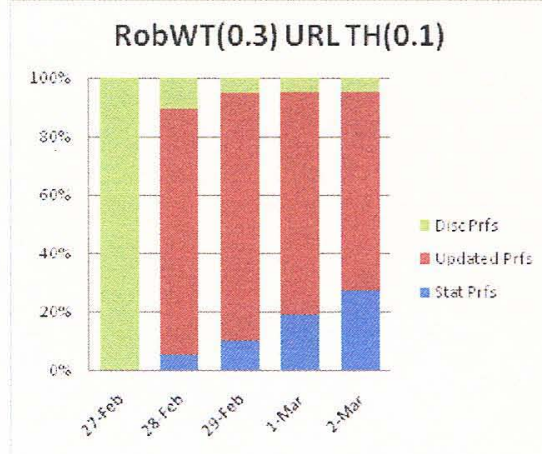

(c)

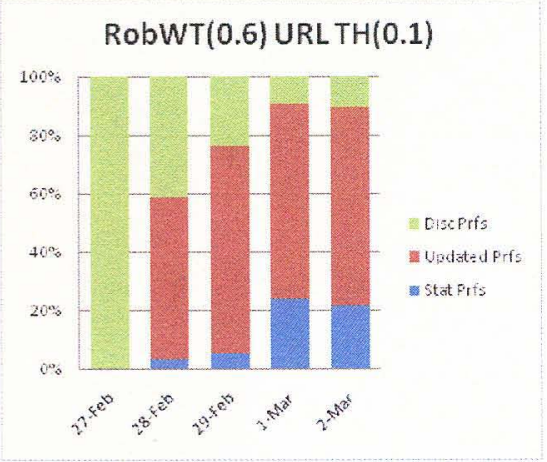

(d)

similarity threshold value of 0.3 , which allows more sessions to match existing profiles and thus update them.

Fig.4.28(b) and (d) show more restricted profile updates, with more new profiles being discovered or old ones remaining the same. This is because the higher value of similarity threshold decreases the chance that a new session would match an existing profile, and hence would either create a new profile if enough such sessions agree on a usage pattern, or would be discarded otherwise.

\subsubsection{Matching vs. Distinct sessions}

For a fixed configuration, the number of matching and distinct sessions may shed 
a light on which time periods have more usage behavior changes. Fig.4.29 shows the percentages of matching and distinct session for each time period when using the binary cosine similarity. All configurations show that at each time period, the percentage of matching sessions was between $15 \%$ and $25 \%$. These results can give a better understanding about the real percentages of profiles and their types as discussed in the previous section, because Fig.4.27 showed that the majority of profiles where updated, which seems to contradict what this figure suggests since the majority of sessions are counted as distinct, so it seems that the majority of profiles should be newly discovered instead of updated. This contradiction is due to the fact that more distinct sessions do not necessary imply more new profiles to be discovered. For example all the distinct sessions might be very close to each other, and thus generate only one distinct profile.

Figure 4.30 shows the number of matching and distinct sessions when using the Robust Weight Similarity. Fig.4.30(a) and (c) show similar behavior where the vast majority of sessions got matched. This is due to the loose similarity threshold value of 0.3, which is compatible with the findings in Fig4.28(a) and (c) where most profiles got updated.

Fig.4.30(b) and (d) show a completely opposite behavior to Fig.4.30(a) and (c) where the majority of sessions are treated as distinct. This is reflected in Fig.4.28(a) and (c) where some profiles were distinct. As discussed above, the large number of distinct sessions does not necessarily mean that a large number of new profiles will be discovered.

The overall trend of matching sessions is shown in Fig.4.31, which shows that for the binary cosine similarity, the percentage of matching sessions is similar for all 
Figure 4.29: U of L Library: Matching vs. Distinct Sessions (Binary Cosine Similarity)

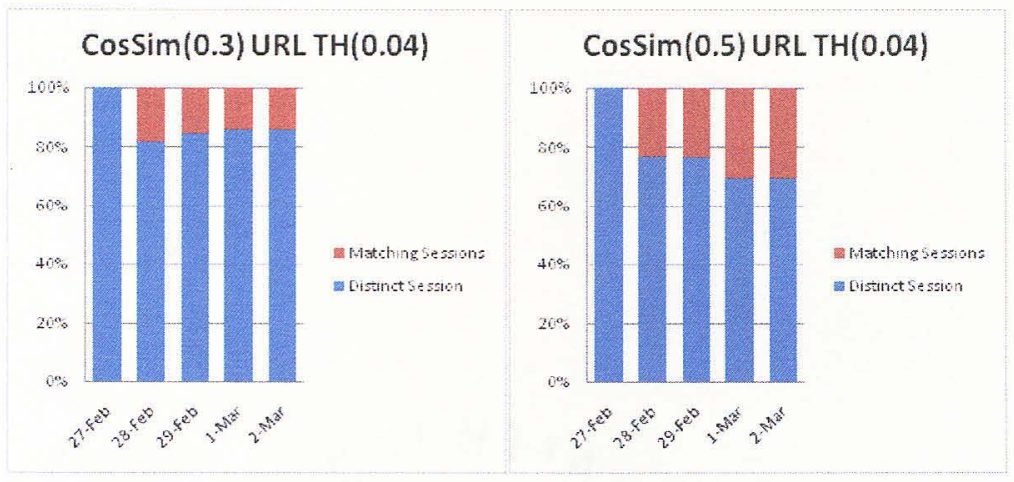

(a)

(b)

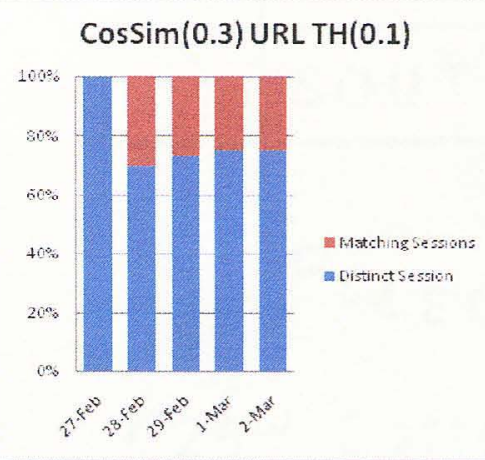

(c)

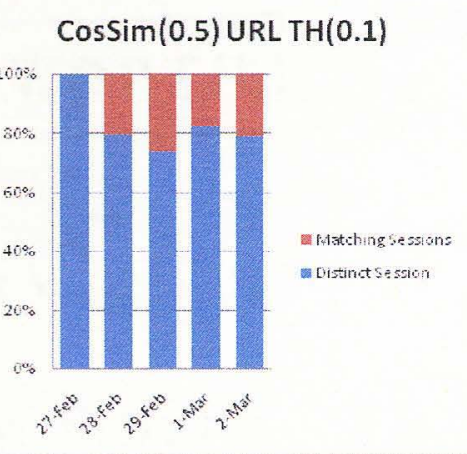

(d) 
Figure 4.30: U of L Library: Matching vs. Distinct Sessions (Robust Weight Similarity)

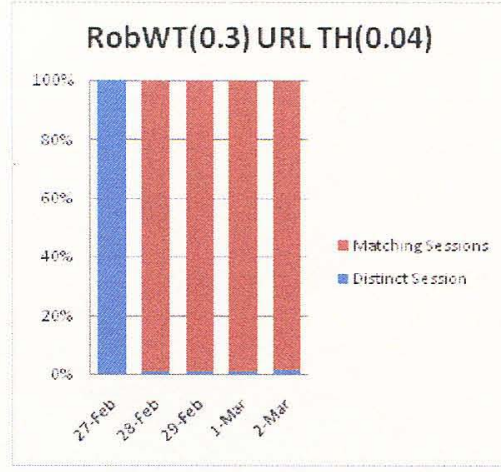

(a)

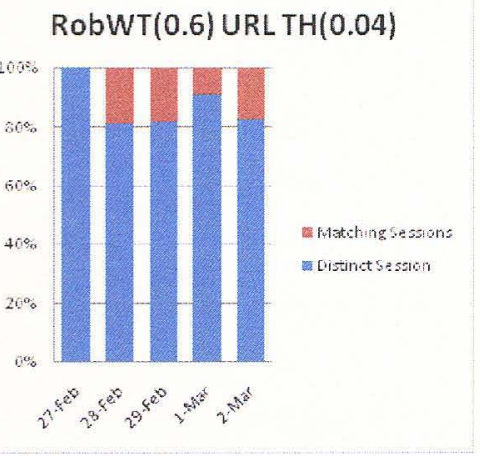

(b)

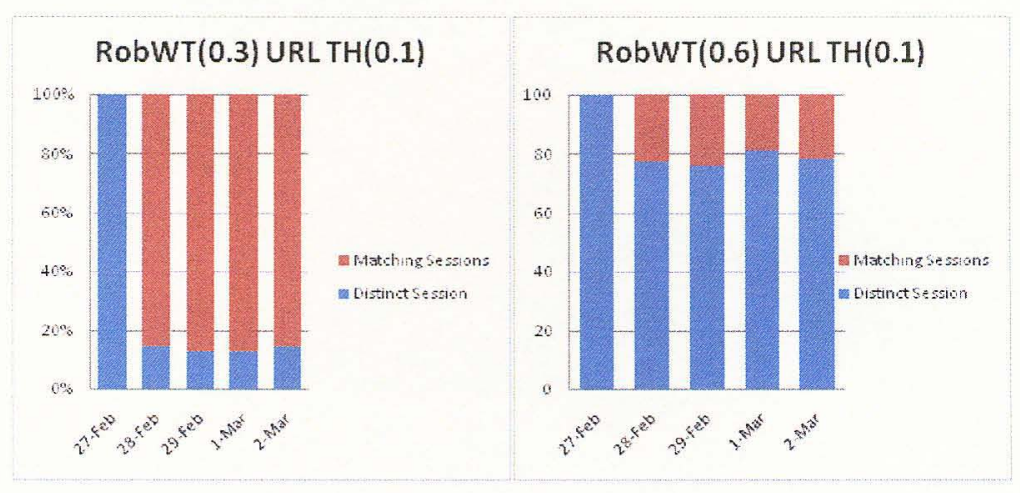

(c)

(d) 
Figure 4.31: U of L Library: Matching Sessions Percentage

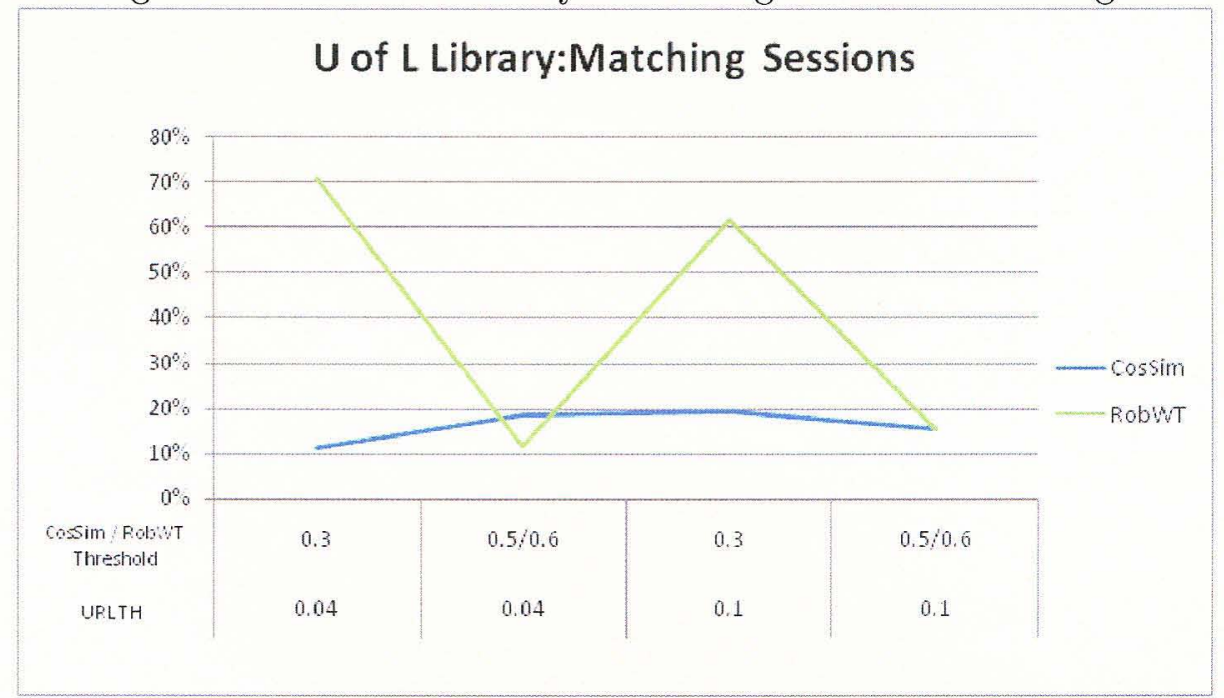

configurations. However, for the robust weight similarity, the percentage gets much higher when the matching threshold is lower.

\subsubsection{Profile Cardinality}

The profile cardinality represents how popular and important a profile is. In Fig.4.32, the cardinality of the profiles with maximum and minimum variance is plotted for each configuration.

Fig.4.32(a) presents the results when using the binary cosine similarity, and it shows that the lower URL weight threshold caused the highest quality profile (i.e. with minimum variance) to have a high cardinality, while a higher URL threshold value caused the high quality profiles to have fewer sessions. However, when it comes to the lowest-quality profile (i.e. the one with high variance), the similarity threshold value was the main factor affecting the cardinality, since a higher threshold causes the profile with maximum variance to acquire more sessions.

Fig.4.32(b) shows the results when using the robust weight similarity. The cardinality of the high quality profile (with low variance) remains the same for all different 
Figure 4.32: U of L Library: Cardinality of Max and Min Sigma

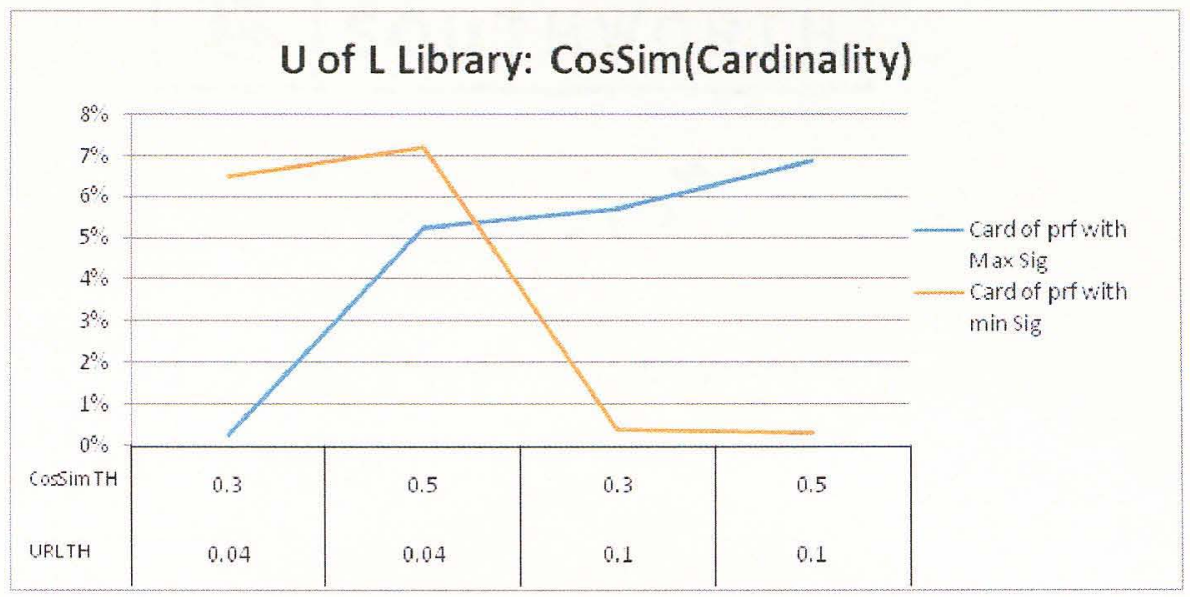

(a)

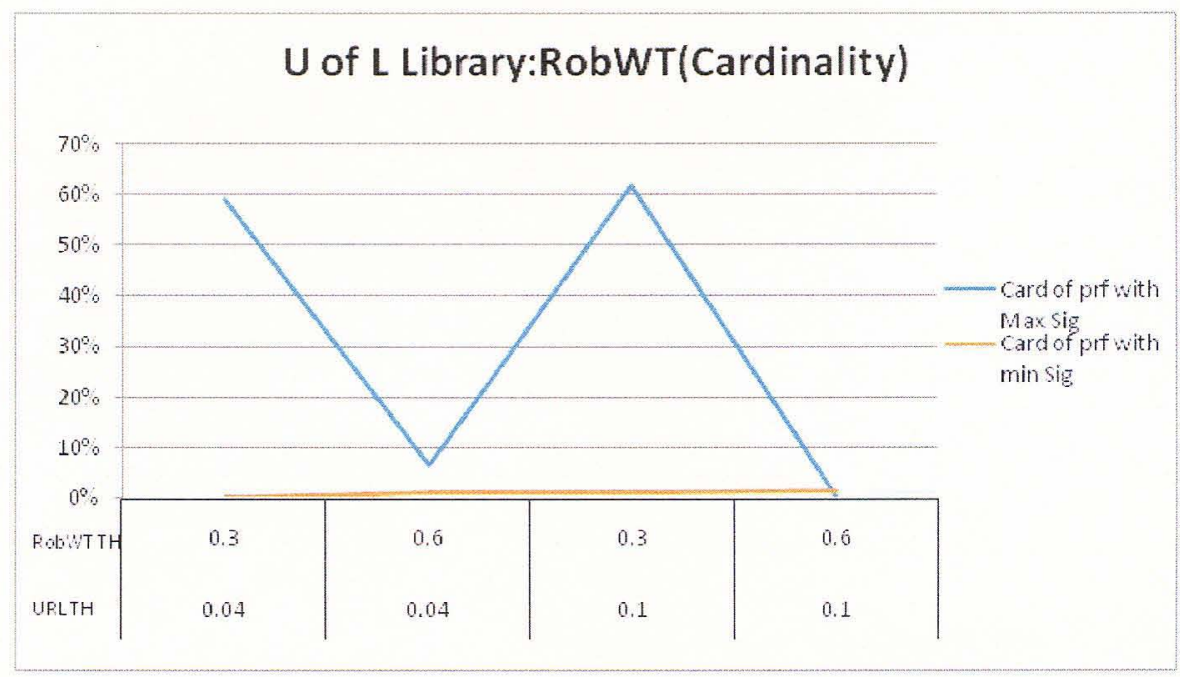

(b)

configurations. However, the cardinality of the profile with maximum variance is sensitive only to the similarity threshold value, where a high threshold value causes the cardinality to decrease, while a lower threshold value causes higher cardinality.

For a more detailed view of the profile quality, the cardinalities of each profile at each time period are plotted. Fig.4.33 shows the cardinality percentage of each profile at each time period when the Binary Cosine Similarity. The cardinality of the 
Figure 4.33: U of L Library: Evolution of Profile Cardinality Percentages (CosSim)

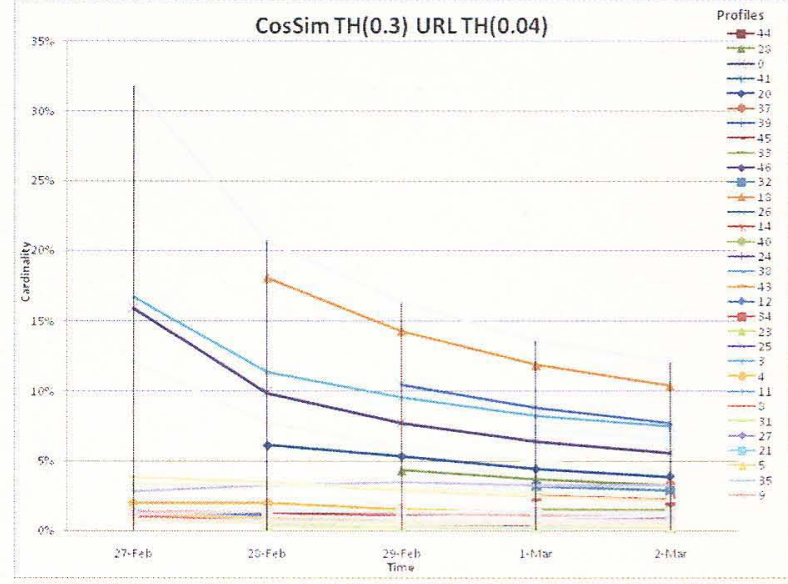

(a)

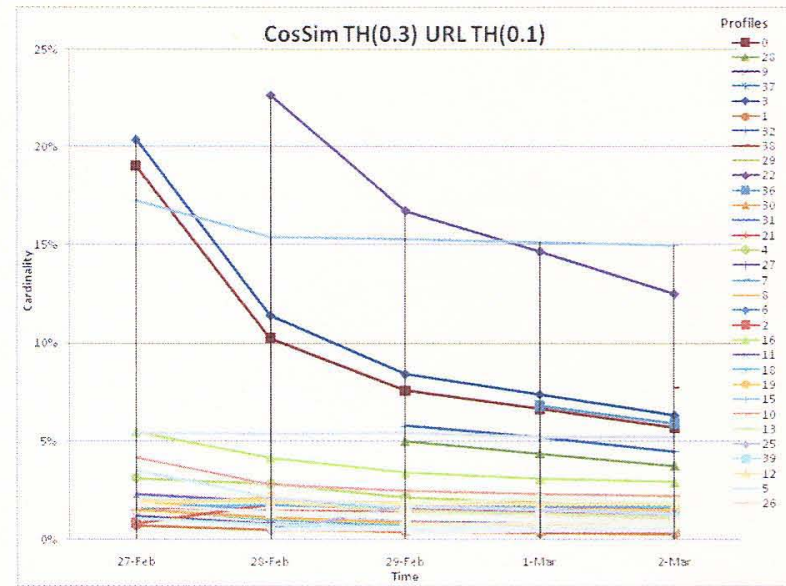

(c)

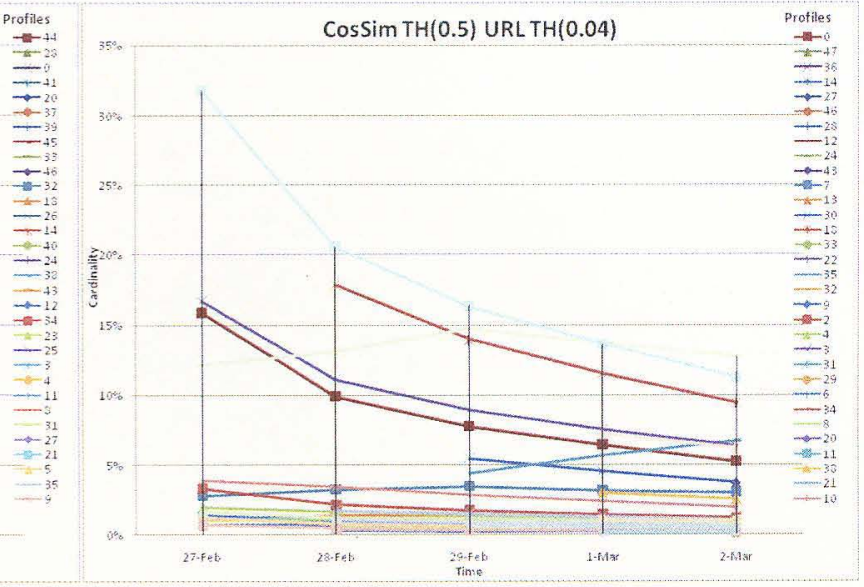

(b)

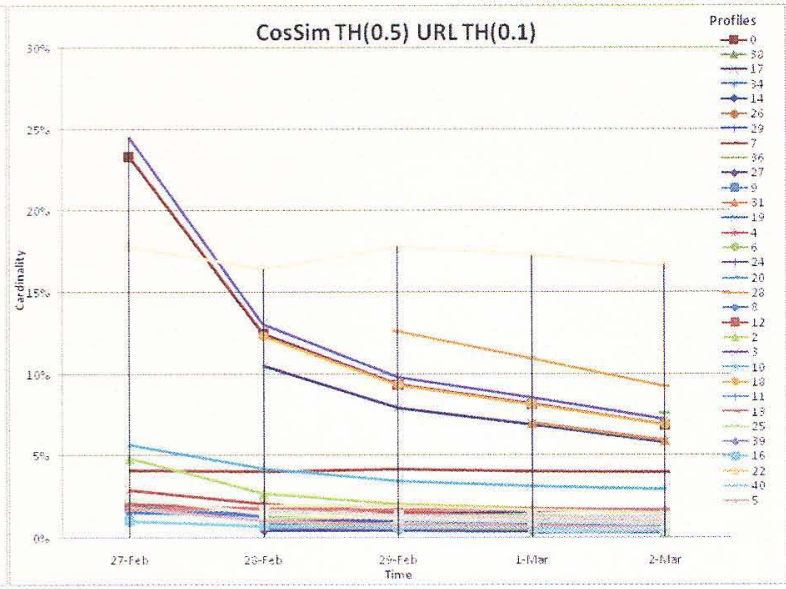

(d)

profile at each time period represents all sessions that matched this profile up to (and including) that time period. The cardinality is always normalized by the sum of all profiles cardinalities. The profiles are ordered (in a decreasing order) based on their variance.

For all configurations, the majority of profiles are slightly decreasing in their cardinality over time. This means that there is a high competition between profiles over new sessions.

Fig.4.34 shows the profile cardinalities over time when using the robust weight 
Figure 4.34: U of L Library: Evolution of Profile Cardinality Percentages (RobWT)

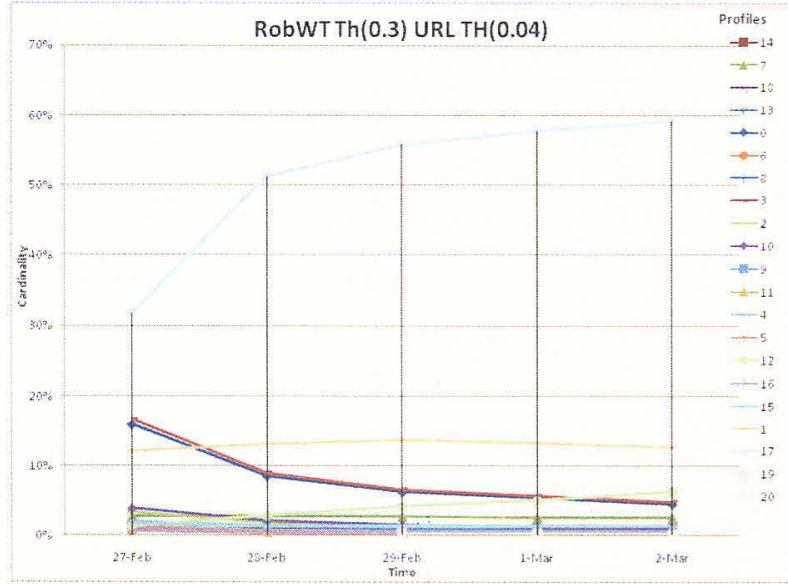

(a)

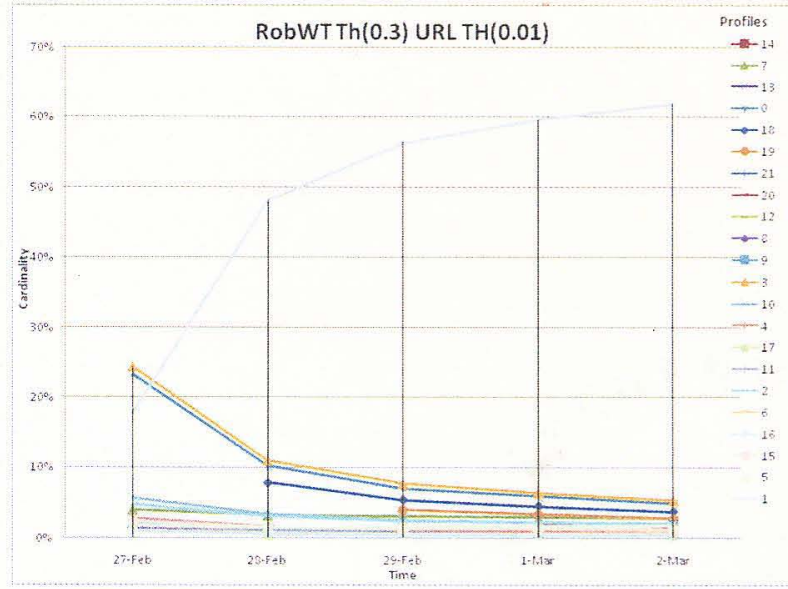

(c)

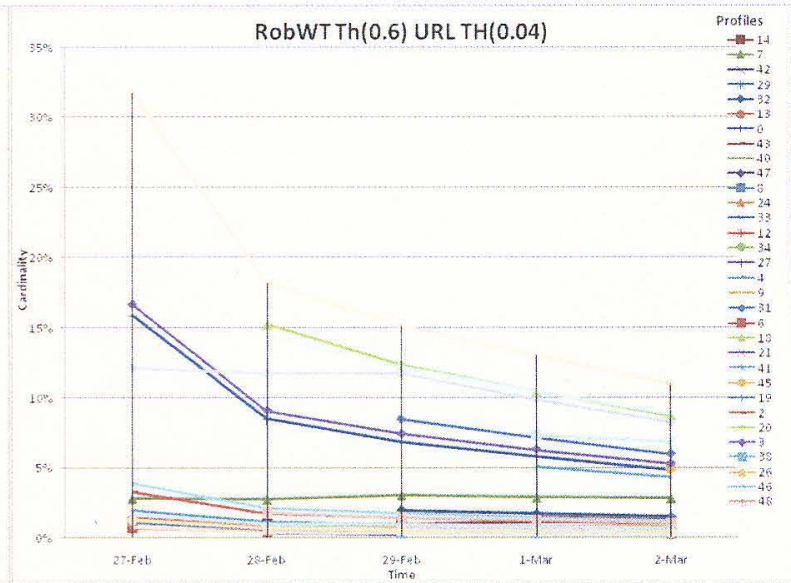

(b)

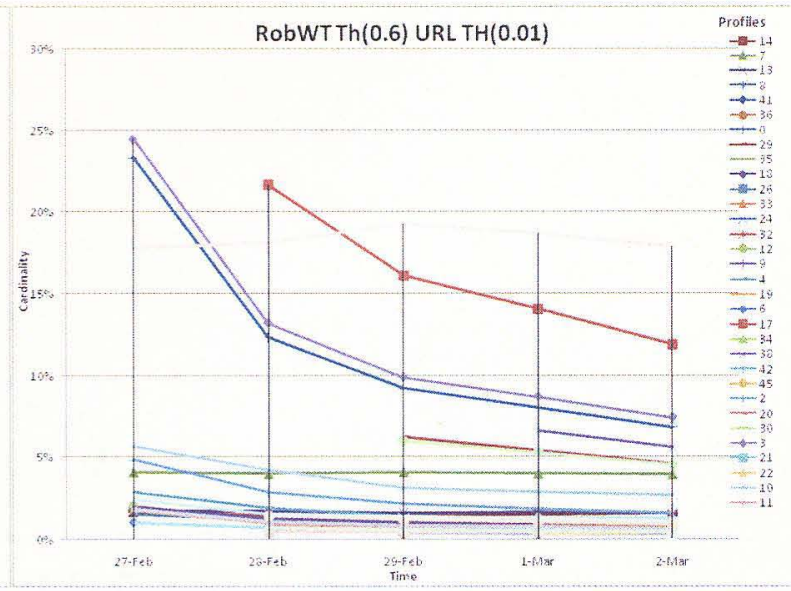

(d)

similarity. As seen in Fig.4.33, the majority of profiles for all configurations slightly decrease their cardinality over time, with the exceptions of two profiles: one in Fig.4.34(a) and one in Fig.4.34(c). Despite the increasing in their cardinality, these two profiles have high variance, which means that they are of low quality; most likely, they are the "default" clusters where all non-matching sessions are assigned.

\subsubsection{Profiles Count}

The number of profiles generated from the usage behavior is shown in Fig.4.35. 
Figure 4.35: U of L Library: Total Profiles Count

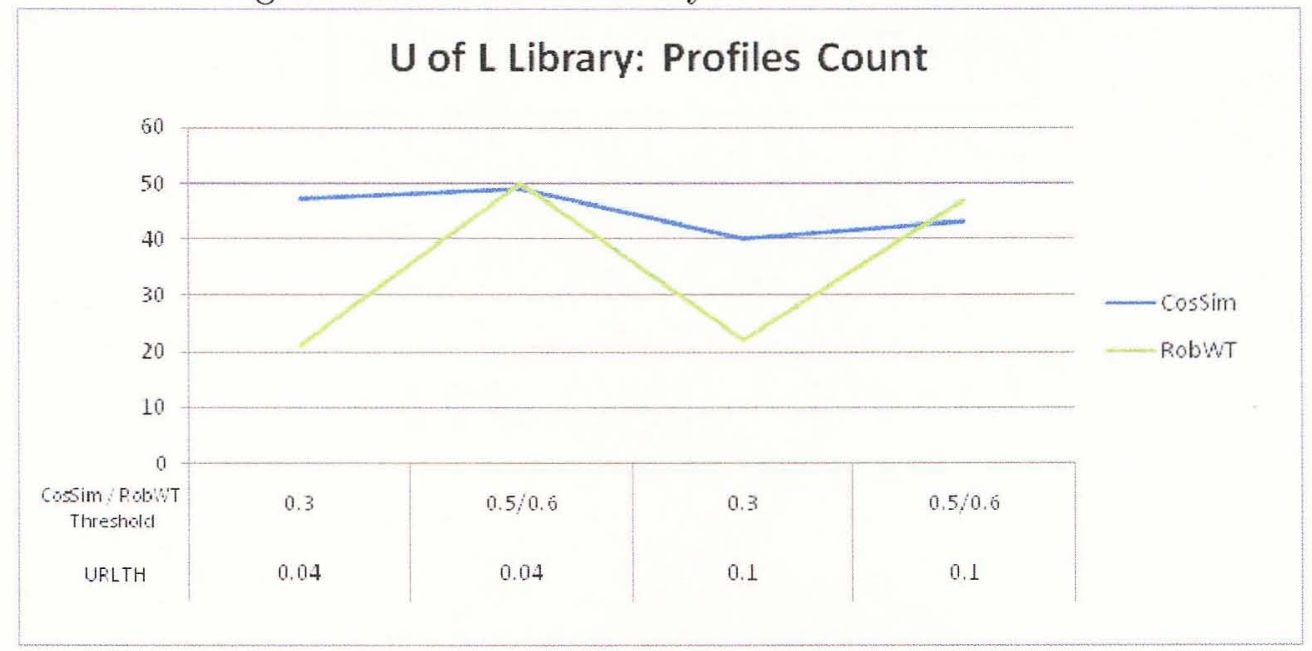

Since HUNC is a hierarchical algorithm, a high number of profiles indicates more detailed profiles, which is desirable. Generally, detailed profiles are of good quality, while a general profile can be considered of low quality, and should be split into multiple detailed profiles. However, the larger number of profiles typically causes low cardinality, since the sessions are distributed over more profiles. Thus, these profiles become more sensitive to any updates and might change their behavior more often.

The number of profiles, when using the binary cosine similarity, is mostly the same for different configurations, whereas using the robust weight threshold generates a lower number of profiles for the lower (0.3) threshold value, and a larger number of profiles for the higher (0.6) threshold value. A more detailed analysis of the profile types was done in Section 4.5.3.

\subsubsection{Profile Pair-wise Similarity}

A powerful evaluation metric is to find how similar the profiles that result from the pattern discovery are to each other. If profiles are too similar to each other, then this would indicate that the updating algorithm was not able to match the new logs 
Figure 4.36: U of L Library: Profile Pair-wise Similarity Aggregates

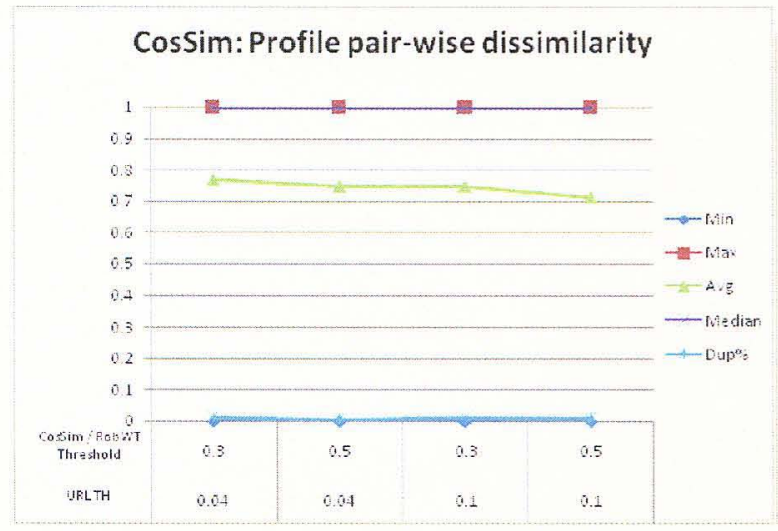

(a)

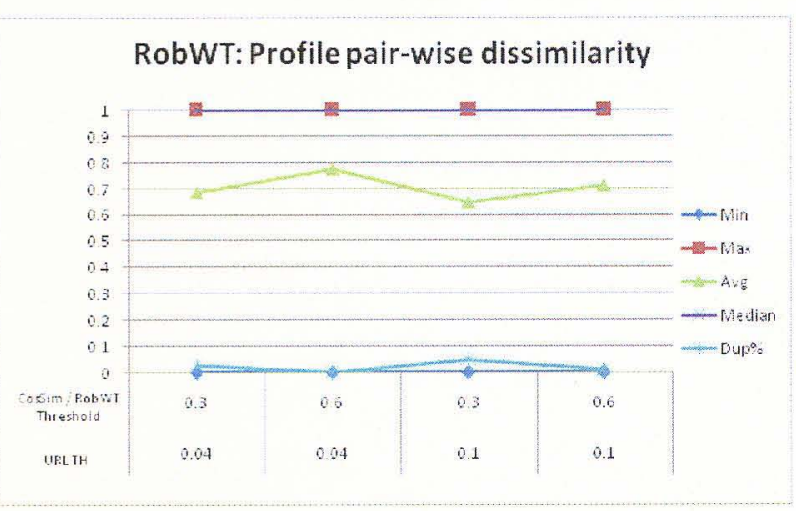

(b)

with the existing profiles accurately.

A difference matrix consisting of the pair-wise similarities between every two profiles at the end of evolution was calculated. The maximum, minimum, average, and median pair-wise similarities are plotted on Fig.4.36, as well as the percentage of duplicate profiles. Duplicate profiles are those whose difference is less than 0.01. This translates to profiles who are at least $90 \%$ similar to each other (using the binary cosine similarity).

Both Figs.4.36(a) and (b) show similar trends, where the maximum and median dissimilarity is one (which means the majority of profiles are completely different), while the average difference is around 0.7 which can be translated to profiles which are only $16 \%$ similar. The minimum profile dissimilarity is close to zero, which means that these two profiles are too similar to each other. The percentage of duplicate profiles is almost zero in all cases (it is zero in some configurations), which means that the total number of duplicate profiles is extremely low, which supports the objective of this thesis of developing high quality, distinct, and evolving profiles through different time periods. 


\subsubsection{Profile Density}

Fig.4.37 shows the profile densities over time when using the Binary Cosine Similarity. The profiles were ordered based on their variance (in decreasing order).

Most profiles maintain a stable density over time for all configurations. Some of them decrease their density slightly as in Fig.4.37(a), which means that the variance is increasing (since the cardinality does not decrease over time), and this increase in variance is due to the profile being updated by new sessions which are not very similar to existing ones in the profile. This happens because of the low similarity threshold value used (0.3).

Fig.4.38 shows the profile densities when using the Robust Weight Similarity. All configurations show better results than the results shown in Fig.4.37 which uses the Binary Cosine Similarity instead. In Fig.4.38 more profiles are improving their quality (increase their density) over time. This supports conclusions reached in figures 4.24 and 4.25 , where the variances are decreasing over time, and profiles are becoming more compact (hence of higher quality). This consistent increase in densities may be the ultimate judge that matching using the Robust Weights (which depend on accurate variance estimates) yields the best quality profiles compared to Cosine Similarity matching.

\subsubsection{Evolution vs. Traditional (Full run)}

The last criterion is the approach of pattern discovery, which is either evolutionary -discussed in this thesis- where the pattern discovery is done through different time periods, or traditional where the log data from all time periods is used in one shot to discover usage patterns. 
Figure 4.37: U of L Library: Evolution of Profile Densities (CosSim)

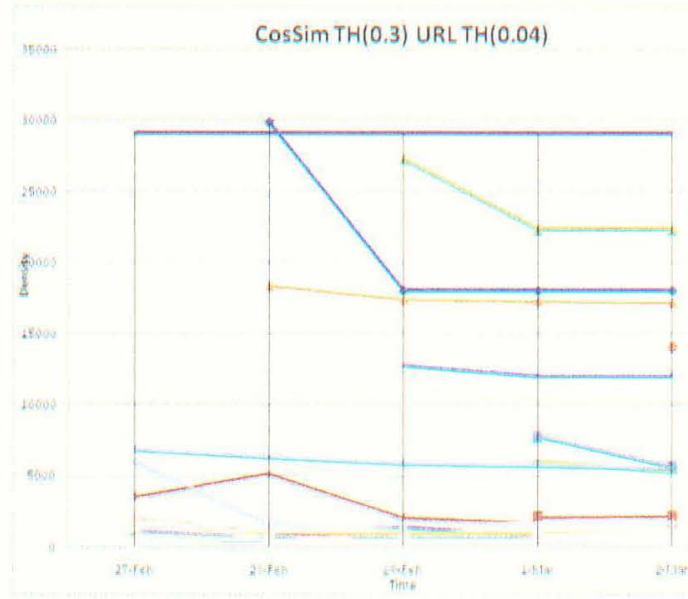

(a)

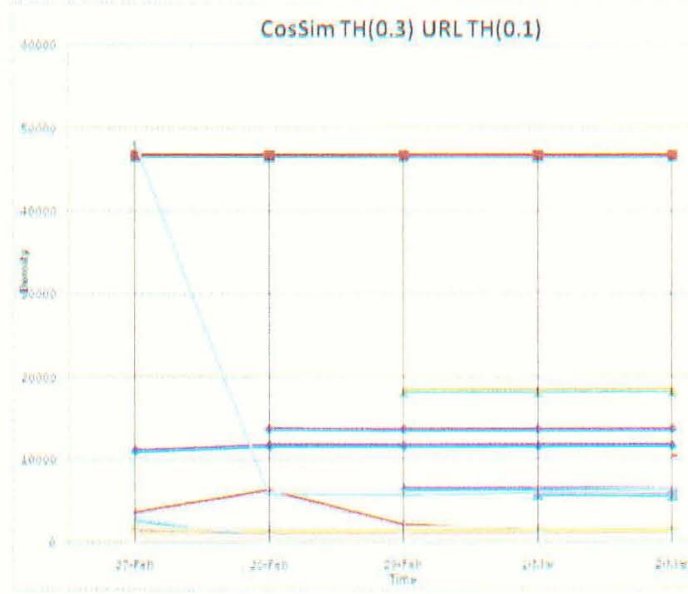

(c)
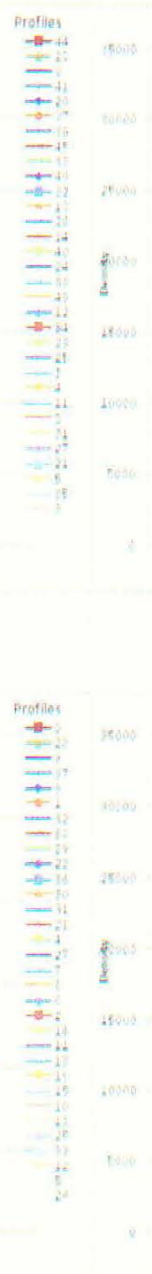

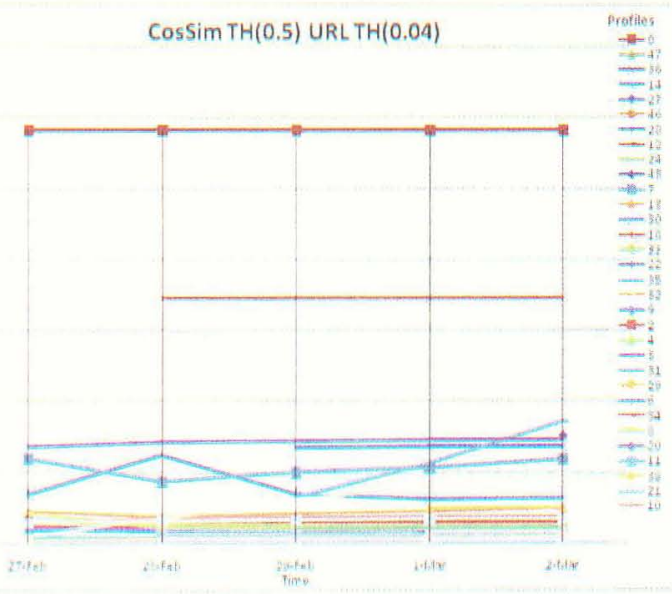

(b)

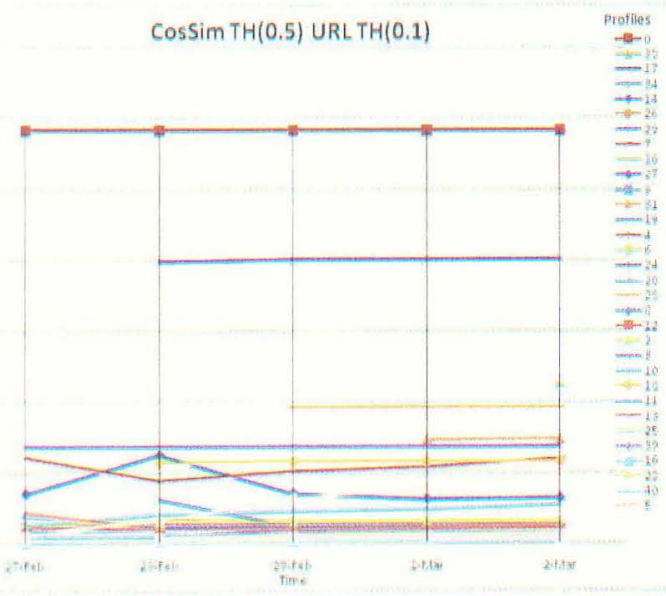

(d) 
Figure 4.38: U of L Library: Evolution of Profile Densities (RobWT)

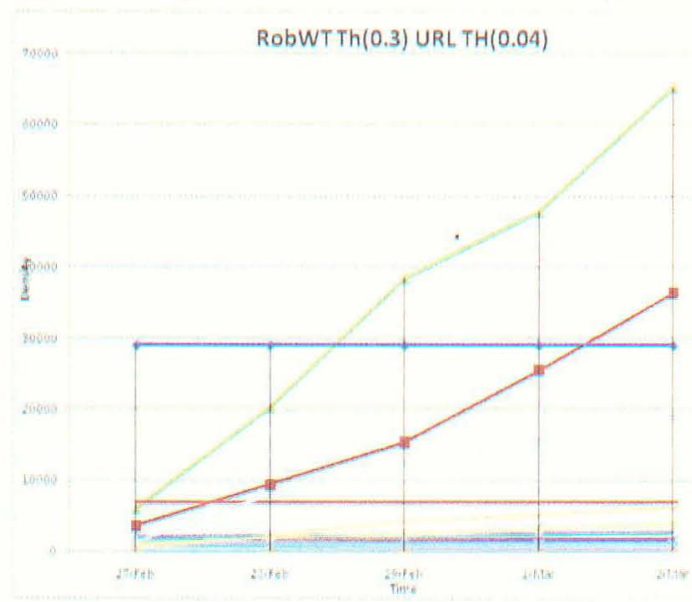

(a)

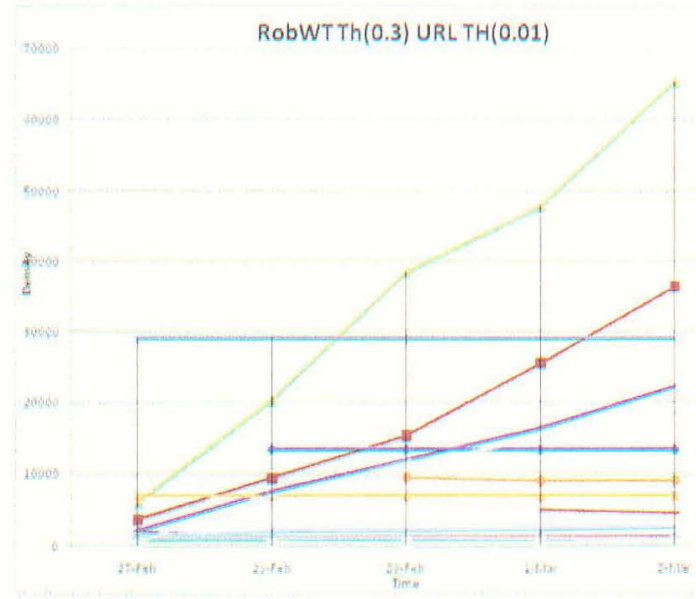

(c)

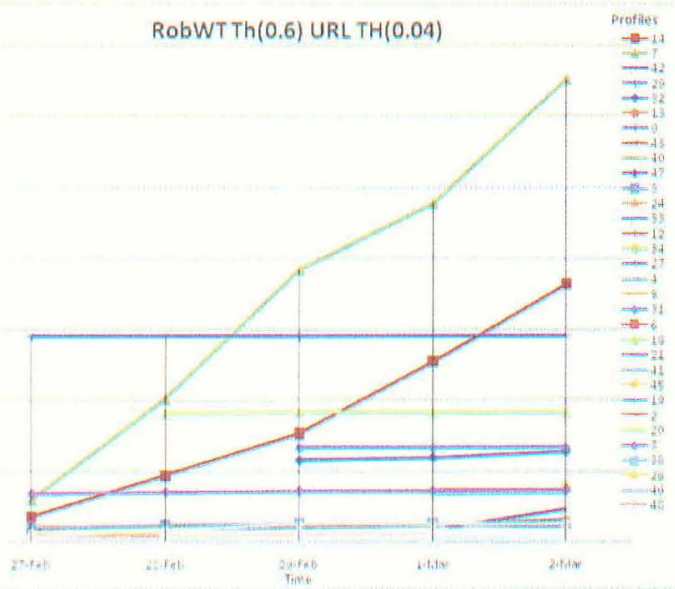

(b)

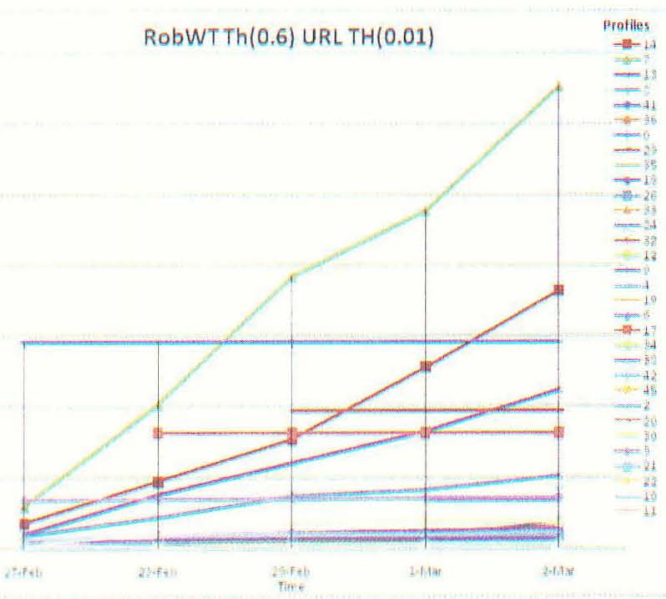

(d) 
Figure 4.39: U of L Library: Traditional Profile Variances

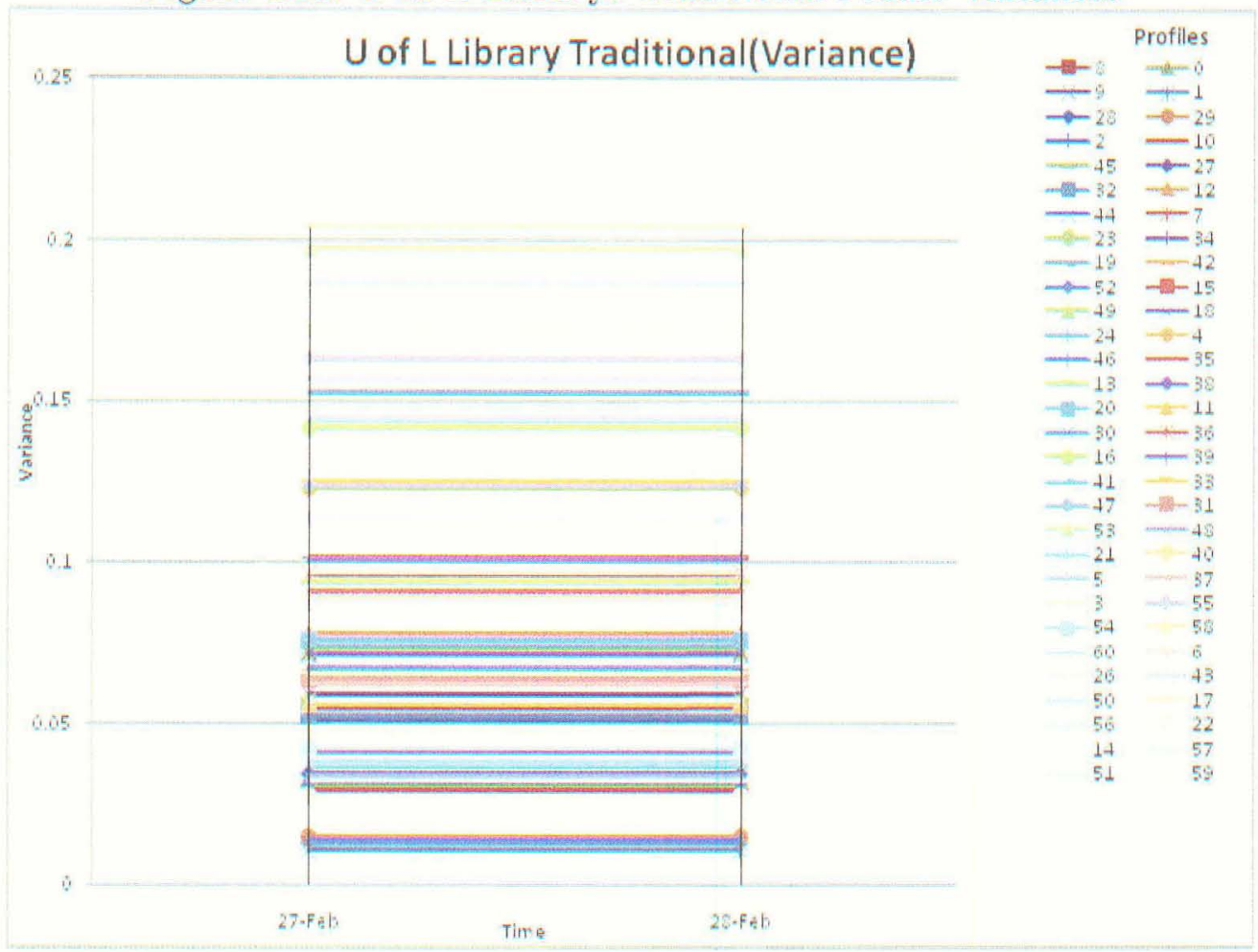

Fig.4.39 shows the profile variances when using the traditional discovery approach. Profiles are ordered in a decreasing order based on their cardinality percentage. All profiles are of good quality since their variance is low, where the highest variance is only about 0.2 .

Fig.4.40 shows the densities of the profiles discovered when using the traditional discovery approach. The density represents the ration between the profile cardinality and its variance. Profiles are order in a decreasing order based on their cardinality percentage. All profiles are of high quality since the density is high. Some of the profiles generated using the evolutionary approach showed a comparable or higher density as was seen in Fig.4.38. 
Figure 4.40: U of L Library: Traditional Profile Density

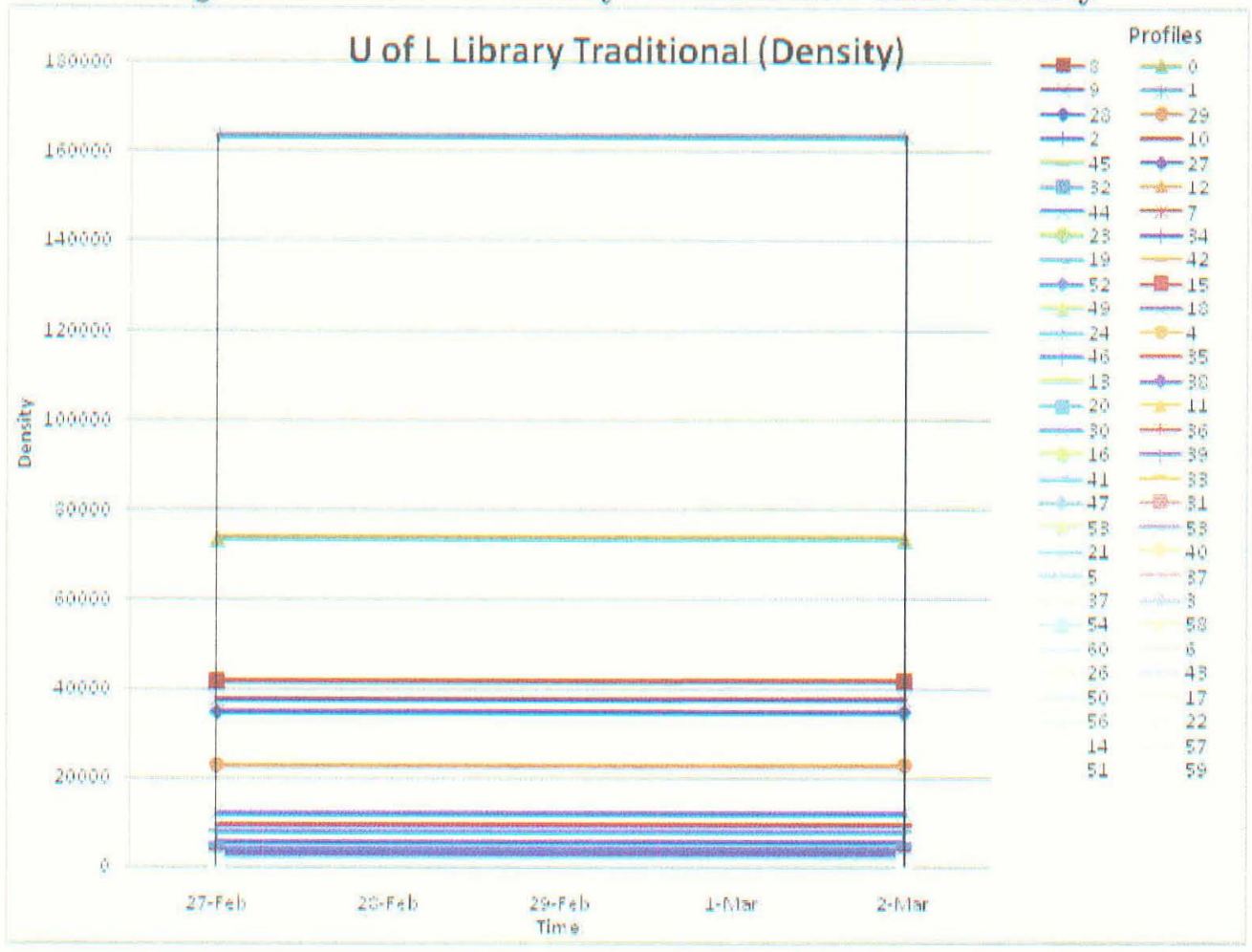

Fig.4.41 compares the aggregate metrics for both discovery approaches (traditional and evolution). The median, maximum, and average variances are less when using the traditional approach than using the evolutionary approach. Fig.4.41(b) shows that using the Robust Weight Similarity in the evolution mode has resulted in the minimum profile variance for all configurations compared to using the Evolution mode's Cosine Similarity and compared to the traditional mode. This means that using the Robust Weight Similarity evolution has discovered profiles (at least one) whose variance is the least over all other approaches. However, this high quality profile might be the result of only a few similar sessions. 
Figure 4.41: U of L Library: Evolution vs Traditional Variance Aggregates

Library: Median Sigma

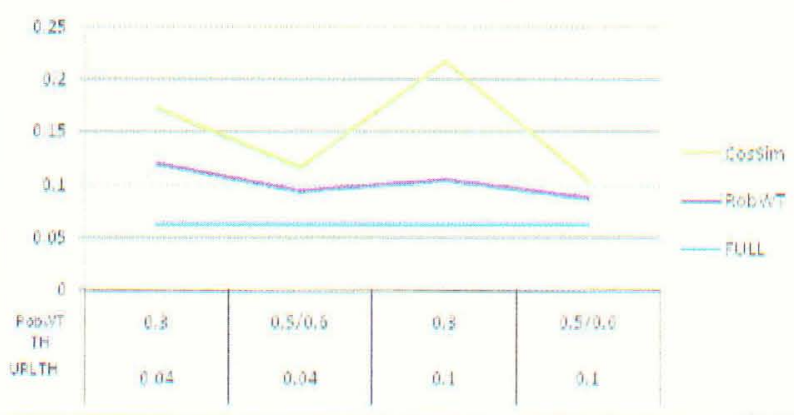

(a)

Library: Maximum Sigma

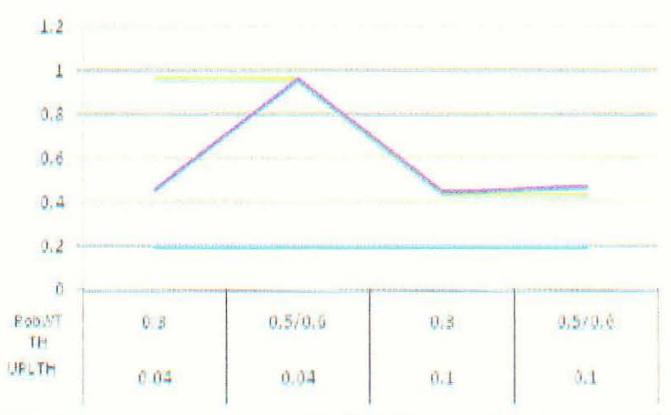

(c)
Library: Minimum Sigma

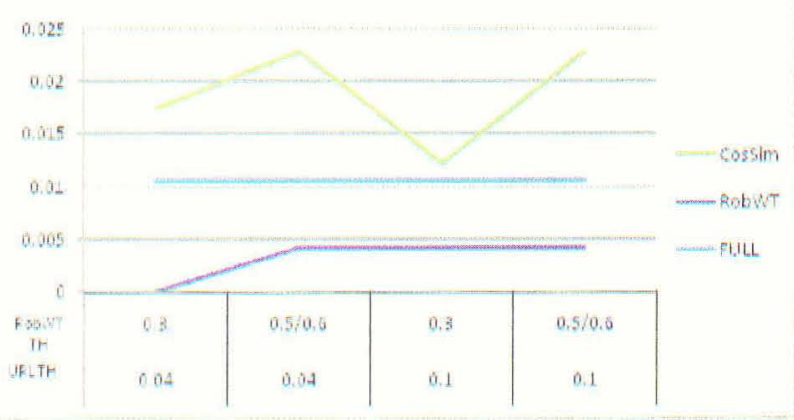

(b)

Library: Average Sigma

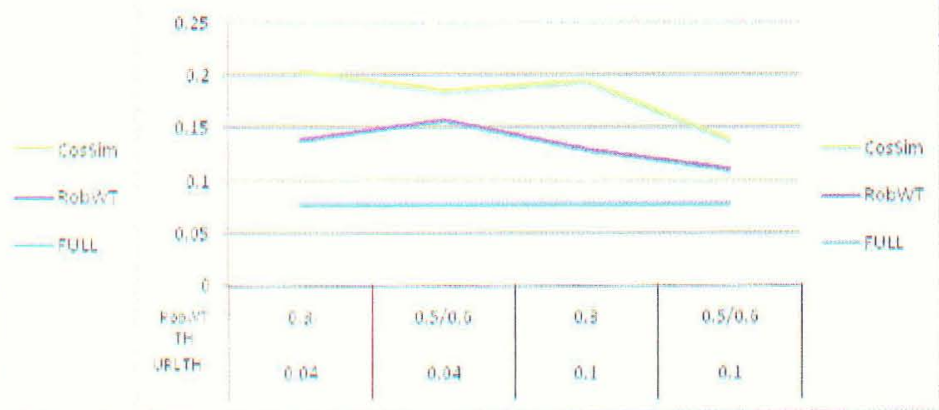

(d) 


\subsection{Experimental Design}

An experimental design analysis was conducted on the University of Louisville dataset to find the significance of the configuration parameters on some of the evaluation metrics.

The factors used in experimental design analysis are listed in Table 4.6. For the "Discovery Approach" factor, the value 0 represents the "Evolution" mode, and 1 represents the "Traditional" discovery mode. The similarity matching method is encoded as 0 for the Binary Cosine Similarity and 1 for the Robust Weight Similarity. The similarity threshold has two levels: 0.3 and 0.55 . During experiments, the value of 0.5 was used as the Binary Cosine Similarity threshold, whereas the value 0.6 was used for the Robust Weight Similarity threshold. However, to have a standardized view of the similarity threshold factor, the average of the two thresholds was used, so as to have only 2 levels instead of 3 . The value of 0.05 is used as the sigma value $\sigma$.

Table 4.7 shows the response variables used in experimental design. Table 4.8 shows the data used in the experimental design analysis.

The results of experimental design, which are in Appendix A, showed that the URL weight threshold value and the profile discovery mode (evolution versus traditional) proved to be the most significant factors on profile variances and cardinalities (with small p-value), the similarity method (Cosine Binary versus Robust Weight) proved significant to some of the response variables (profile variances and densities), and the similarity threshold proved significant to some of the response variables (profile variances and number of profiles). 
Table 4.6: Experimental Design Factors

\begin{tabular}{|c|c|c|}
\hline Factor & Number of Levels & Level Values \\
\hline \hline Discovery Approach & 2 & 0,1 \\
\hline Similarity Matching Method & 2 & 0,1 \\
\hline URL Weight Threshold & 2 & $0.04,0.1$ \\
\hline Similarity Threshold & 2 & $0.3,0.55$ \\
\hline
\end{tabular}

Table 4.7: Experimental Design Responses

\begin{tabular}{|c|c|}
\hline Response & Level Values \\
\hline \hline Max Variance & {$[0-1]$} \\
\hline Average variance & {$[0-1]$} \\
\hline Number of Profiles & {$[1-\#$ sessions $]$} \\
\hline Cardinality \% of Profile with Max Variance & $(0-1]$ \\
\hline Percentage of Duplicate Profiles & {$[0-1]$} \\
\hline Profiles Density Average & {$[1-\infty]$} \\
\hline
\end{tabular}

Table 4.8: Experimental Design Data Table

\begin{tabular}{|c|c|c|c|c|c|c|c|c|c|}
\hline $\begin{array}{c}\text { Disc. } \\
\text { Approach }\end{array}$ & $\begin{array}{l}\text { Sim. Match. } \\
\text { Method }\end{array}$ & $\begin{array}{l}\text { URL Wt } \\
\text { Thr }\end{array}$ & Sim.Thr & Max var & Avg. Var & $\begin{array}{l}\text { Num. } \\
\text { Profides }\end{array}$ & $\begin{array}{l}\text { Card \%of } \\
\text { Prf with } \\
\text { max var. }\end{array}$ & Dupl. $\%$ & Den Avg. \\
\hline 0 & 0 & 0.04 & 0.30 & 0.966092 & 0.203 & 47 & 6.5 & 0.0120259 & 35536 \\
\hline 0 & 0 & 0.04 & 0.55 & 0.9151000 & 0185 & 45 & 7.2 & 0.0026596 & 2442.2 \\
\hline 0 & 0 & 0.10 & 0.30 & 0.435975 & 0.194 & 40 & 0.4 & 0.0102564 & 3467.3 \\
\hline 0 & 0 & 0.10 & 0.55 & 0.428800 & 0.138 & 43 & 0.3 & 0.0066445 & 2975.0 \\
\hline 0 & 1 & 0.04 & 0.30 & 0.460281 & 0139 & 21 & 593 & 0.0285714 & 9380.6 \\
\hline 0 & 1 & 0.04 & 0.55 & 0.962000 & 0.158 & 50 & 6.7 & 0.0048980 & 4998.6 \\
\hline 0 & 1 & 0.10 & 0.30 & 0.448265 & 0.129 & 22 & 619 & 0.0519481 & 101868 \\
\hline 0 & 1 & C. 10 & 0.55 & 0.475400 & 0.111 & 47 & 0.3 & 0.0120259 & 5671.9 \\
\hline 1 & of & 0.04 & 0.30 & 0.203900 & 0078 & 61 & 0.4 & 0.0018939 & 7867.0 \\
\hline 1 & 0 & 0.04 & $0.5 E$ & 0203900 & 0078 & $6 !$ & 0.4 & 0.0018939 & 7867.0 \\
\hline 1 & 0 & 0.10 & 0.30 & 0.203900 & 0.078 & 61 & 0.4 & 0.0018939 & 7867.0 \\
\hline 1 & 0 & 0.10 & 0.55 & 0.203900 & 0.078 & SI & 0.4 & 0.0018939 & 78670 \\
\hline 1 & 1 & 0.04 & 0.30 & 0.203900 & 0078 & 61 & 04 & 0.0018939 & 7867.0 \\
\hline 1 & 1 & 004 & 0.5 .5 & 0.203900 & 0078 & 61 & 04 & 0.0018939 & 7867.0 \\
\hline 1 & 1 & 0.10 & 0.30 & 0.203900 & 0.078 & 61 & 0.4 & 0.0018939 & 7867.0 \\
\hline 1 & $\$$ & 0.10 & $0.5 E$ & 0.203900 & 0.079 & 51 & 0.4 & 00018939 & 7867.0 \\
\hline
\end{tabular}




\subsection{Summary}

Chapter 4 presented the results of applying the proposed framework on two datasets: University of Missouri CECS department's website logs, and University of Louisville's Library website logs. Each of the evaluation metrics and the proposed framework's parameters were discussed.

The results of the experiments showed that using the Robust Weight Similarity with a high similarity threshold value of 0.6 , and a strict $\mathrm{CRL}$ weight threshold of 0.1 , gives the best results. The compactness of the profiles for this configuration kept improving over time, as seen in Figures 4.4 and 4.25. A lower profile variance means that the sessions are more similar to the profile. The higher quality of the profiles has made these profiles able to match more incoming new sessions, as seen in figures $4.7(\mathrm{~d})$ and $4.28(\mathrm{~d})$, where the majority of the profiles got updated. Moreover, this configuration resulted in more detailed profiles, where the average number of profile was around 20 when mining the University of Missouri CECS department's dataset and around 50 when mining the University of Louisville's Library dataset, as seen in Figures 4.14 and 4.35 respectively. These detailed profiles were also very different from each other (based on the profile pair-wise similarity measure), as seen in Figures $4.15(\mathrm{~b})$ and $4.36(\mathrm{~b})$, where the percentage of duplicate profiles was almost 0 for both cases. The profile quality using this configuration was further confirmed when the density of profiles was found to be increasing over time, as seen in Figures 4.17 and 4.38. The consistent increase in densities may be the ultimate judge that matching using the Robust Weights (which depend on accurate variance estimates) yields the best quality profiles compared to Cosine Similarity matching.

The results of experimental design showed that the URL weight threshold value and the profile discovery mode (evolution versus traditional) proved to be the most significant factors on profile variances and cardinalities (with small p-value), the similarity method (Cosine Binary versus Robust Weight) proved significant to some of 
the response variables (profile variances and densities), and the similarity threshold proved significant to some of the response variables (profile variances and number of profiles). 


\section{CHAPTER 5}

\section{CONCLUSION}

The increasing importance of the user has challenged online organizations to start tracking the behavior of their website users on the web, in order to better understand and satisfy their needs, and hence, maintain the loyalty of current users and lure new users. Since web usage data tends to be huge, heterogeneous, and continuously growing and changing, traditional data management tools are neither feasible nor cost-

effective to handle them. Web usage mining is the process of discovering interesting usage behavior over the World Wide Web. Many studies have been conducted to adapt web usage mining to business needs, however, very few have tried to handle the changing nature of the web user activities.

This thesis has presented an innovative and scalable framework that is capable of capturing the changing behavior of users over the World Wide Web. The proposed framework develops a set of evolving profiles that represent the usage pattern as a set of URLs with varying degrees of significance. These profiles can be considered as representatives of usage behavior at any given time. The proposed framework is applied each time that new data becomes available. It tries to match these transactions with previous user behavior (as in collaborative filtering), uses matching transactions to update the old profiles, and subjects new (non-matching) transactions to a new pattern discovery process using the Hierarchical Lnsupervised Niche Clustering (HUNC) 
algorithm.

The resulting profiles are formulated in way that makes it intuitive to utilize them in higher-level applications to meet business goals such as web recommendations, web personalization, and user-driven web content. These evolving profiles also allow a better understanding of the changing interests of visitors to a website.

The proposed framework was applied on the web server logs of two real web sites: University of Missouri's CECS department website, and University of Louisville's Library website. The developed usage profiles were evaluated using a set of metrics including the profile variance (how much the sessions in a cluster are dispersed or different from each other), profile cardinality (how many sessions the profile represents), the percentage of matching transactions (to which degree are the new transactions similar to older profiles), profiles' pair-wise similarity (how similar the developed profiles are to each other), the profile density (which is the ratio of the profile variance and cardinality), and the total number of profiles and their types (new, distinct, or static).

To find high quality profiles, several parameters were varied within different configurations. These parameters include the matching method which could be the Binary Cosine Similarity or the Robust Weight Similarity (which is variance-sensitive), the similarity threshold value which controls how strict this matching is, and the URL significance weight threshold which controls the quality of URLs allowed in the profile. Moreover, the profiles resulting from the evolution mode of discovery were compared to the traditional mode, where all the available data is mined in one shot.

The experiments have showed that the output quality should be evaluated by scrutinizing the different parameter configurations, and observing the evaluation metrics from different perspectives, because there is always a trade-off between different quality measures. For example, an increase in the profile cardinality (number of sessions assigned to the profile) might cause a decrease in the profile's compactness (i.e. higher 
variance), which may not be desirable.

The results of the experiments also showed that using the Robust Weight Similarity with a high similarity threshold value of 0.6 , and a strict URL weight threshold of 0.1 , gives the best results. The compactness (variance) of the profiles for this configuration kept improving over time, the higher quality of the profiles has made them match more incoming new sessions, more detailed profiles were discovered, and those detailed profiles were also very different from each other (as verified by the low profile pair-wise similarity measures). The profile quality using this configuration was further confirmed when the density of profiles was found to be increasing over time. The consistent increase in densities may be the ultimate judge that matching using the Robust Weights (which depend on accurate variance estimates) yields the best quality profiles compared to Cosine Similarity matching.

The proposed framework's output patterns were comparable to the ones resulting from the traditional pattern discovery mode. The latter can be considered as the best output possible since all usage data is mined at once (instead of at different time periods as in the proposed approach). Having said that, the proposed framework has still shown desired behavior and resulted in high quality profiles that are good representatives of the users' browsing behavior at any given time. Most importantly, the proposed framework has the critical advantage of enabling scalability in handling very large usage data that makes it impossible to mine all patterns in one shot.

The results of experimental design showed that the URL weight threshold value and the profile discovery mode (evolution versus traditional) proved to be the most significant factors on profile variances and cardinalities (with small p-value), the similarity method (Cosine Binary versus Robust Weight) proved significant to some of the response variables (profile variances and densities), and the similarity threshold proved significant to some of the response variables (profile variances and number of profiles). 
This thesis has achieved its objectives, and succeeded in providing the means to track usage changes over the web. However, this framework can be improved further. As part of future work, experiments on more challenging datasets could be conducted, and more parameters, such as HUNC parameters, could be studied in the evaluation experiments. A forgetting factor could also be considered so that new data would have more importance compared to older data as in [7]. Also, developing a high-level application, such as a recommendation system, that uses the generated profiles could give more insight when evaluating the performance of proposed framework. 


\section{REFERENCES}

[1] R. Cooley, B. Mobasher, and J. Srivastava. Web Mining: Information and Pattern discovery on the World Wide Web, Proc. IEEE Intl. Conf. Tools with AI, Newport Beach, CA, pp. 558-567, 1997.

[2] O. Nasraoui and R. Krishnapuram, and A. Joshi. Mining Web Access Logs Using a Relational Clustering Algorithm Based on a Robust Estimator, 8th International World Wide Web Conference, Toronto, pp. 40-41, 1999.

[3] J. Srivastava, R. Cooley, M. Deshpande. and P-N Tan, Web usage mining: Discovery and applications of usage patterns from web data, SIGKDD Explorations, Vol. 1, No. 2, Jan 2000, pp. 1-12.

[4] M. Spiliopoulou and L. C. Faulstich. WUM: A Web utilization Miner, in Proceedings of EDBT workshop WebDB98, Valencia, Spain, 1999.

[5] T. Yan, M. Jacobsen, H. Garcia-Molina, and U. Dayal. From user access patterns to dynamic hypertext linking. In Proceedings of the 5th International World Wide Web conference, Paris, France, 1996.

[6] O. Nasraoui and R. Krishnapuram. A New Evolutionary Approach to Web Usage and Context Sensitive Associations Mining, International Journal on Computational Intelligence and Applications - Special Issue on Internet Intelligent Systems, Vol. 2, No. 3, pp. 339-348, Sep. 2002. 
[7] O. Nasraoui, C. Cardona, C. Rojas, and F. Gonzalez. Mining Evolving User Profiles in Noisy Web Clickstream Data with a Scalable Immune System Clustering Algorithm, in Proc. of WebKDD 2003, Washington DC, Aug. 2003, 71-81.

[8] P. Desikan and J. Srivastava, Mining Temporally Evolving Graphs. In Proceedings of "WebKDD- 2004 workshop on Web Mining and Web Usage Analysis", B. Mobasher, B. Liu, B. Masand, O. Nasraoui, Eds. part of the ACM KDD: Knowledge Discovery and Data Mining Conference, Seattle, WA, 2004.

[9] O. Nasraoui, C. Rojas, and C. Cardona, A Framework for Mining Evolving Trends in Web Data Streams using Dynamic Learning and Retrospective Validation, in Computer Networks, Special Issue on Web Dynamics, 50(14), Oct., 2006.

[10] I. Grabtree and S. Soltysiak, Identifying and Tracking Changing Interests. International Journal of Digital Libraries, vol. 2, 38-53.

[11] I. Koychev, Gradual Forgetting for Adaptation to Concept Drift. In Proceedings of ECAI 2000 Workshop Current Issues in Spatio-Temporal Reasoning. Berlin, Germany, 2000, pp. 101-106

[12] O. Nasraoui, M. Soliman, E. Saka, A. Badia, R. Germain, A Web Usage Mining Framework for Mining Evolving user Profiles in Dynamic web Sites, IEEE Transactions on Knowledge and Data Engineering, Feb. 2008, pp 202-215

[13] A. Maloof, S. Michalski, Learning Evolving Concepts Using Partial-Memory Approach. Working Notefs of the 1995 AAAI Fall Symposium on Active learning, Boston, MA. Pp 70-73

[14] Nasraoui O., and Krishnapuram R., "A Novel Approach to Unsupervised Robust Clustering using Genetic Niching," Proc. of the 9th IEEE International Conf. on Fuzzy Systems, San Antonio, TX, May 2000, pp. 170-175. 
[15] L. Catledge and J. Pitkow. Characterizing browsing behaviors on the World Wide Web. Computer Networks and ISDN Systems, 27(6), 1995.

[16] J. H. Holland, Adaption in Natural and Artificial Systems (MIT Press, 1975)

[17] O. Nasraoui, C. Cardona-Uribe, C. Rojas-Coronel, "TECNO-Streams": tracking evolving clusters in noisy data streams with a scalable immune system learning model, in: IEEE International Conference on Data Mining, Melbourne, Florida, November 2003.

[18] J. Pitkow and K.Bharat. Webviz: A tool for world-wide web access log analysis. In First International WWW Conference, 1994.

[19] B. Mobasher, N. Jain, E.Han, and J. Srivastava. Web mining: Pattern discovery from world wide web transactions. Technical Report TR 96-050, University of Minnesota, Dept. of Computer Science, Minneapolis, 1996

[20] R. Sarukkai, Link prediction and path analysis using Markov chains, Computer Networks Volume 33, Issues 1-6, June 2000, Pages 377-386

[21] R. Agrawal and R. Srikant, "Mining sequential patterns," p. 3, 11th International Conference on Data Engineering (ICDE'95), 1995

[22] P.-N. Tan, M. Steinbach, and V. Kumar, Introduction to Data Mining, Addison Wesley, 2005.

[23] R.Baraglia and F. Silvestri, "An Online Recommender System for Large Web Sites" IEEE/WIC/ACM International Conference on Web Intelligence (WI'04), pp. 199-205, 2004.

[24] Agrawal, R. and Srikant, R. Fast Algorithms for Mining Association Rules in Large Databases. In Proceedings of the 20th international Conference on Very Large Data Bases, p. 487-499, Sep. 1994. 
[25] J Borges and M Levene, Data Mining of User Navigation Patterns, in Proc. of WebKDD 2000, KDD workshop on Web Usage Analysis and User Profiling, 2000

[26] M Spiliopoulou, C Pohle, and LC Faulstich, Improving the Effectiveness of a Web Site with Web Usage Mining, In Proc. of WebKDD 2000, KDD workshop on Web Usage Analysis and User Profiling, 2000. 


\section{APPENDIX A}

\section{Max Variance:}

General Linear Model: Sigma Max versus Method, Matching Method, ...

$\begin{array}{llrl}\text { Factor } & \text { Type } & \text { Levels } & \text { Values } \\ \text { Method } & \text { fixed } & 2 & 0,1 \\ \text { Matching Method } & \text { fixed } & 2 & 0,1 \\ \text { URL } & \text { fixed } & 2 & 0.04,0.10 \\ \text { Matching Sim } & \text { fixed } & 2 & 0.30,0.55\end{array}$

Analysis of Variance for Sigma Max, using Adjusted SS for Tests

$\begin{array}{lrrrrrr}\text { Source } & \text { DF } & \text { Seq SS } & \text { Adj SS } & \text { Adj MS } & \text { F } & \text { P } \\ \text { Method } & 1 & 0.76852 & 0.76852 & 0.76852 & 26.92 & 0.000 \\ \text { Matching Method } & 1 & 0.01243 & 0.01243 & 0.01243 & 0.44 & 0.523 \\ \text { URL } & 1 & 0.15228 & 0.15228 & 0.15228 & 5.33 & 0.041 \\ \text { Matching Sim } & 1 & 0.01668 & 0.01668 & 0.01668 & 0.58 & 0.461 \\ \text { Error } & 11 & 0.31402 & 0.31402 & 0.02855 & & \\ \text { Total } & 15 & 1.26393 & & & & \end{array}$

$\mathrm{S}=0.168959 \quad \mathrm{R}-\mathrm{Sq}=75.16 \% \quad \mathrm{R}-\mathrm{Sq}(\operatorname{adj})=66.12 \%$

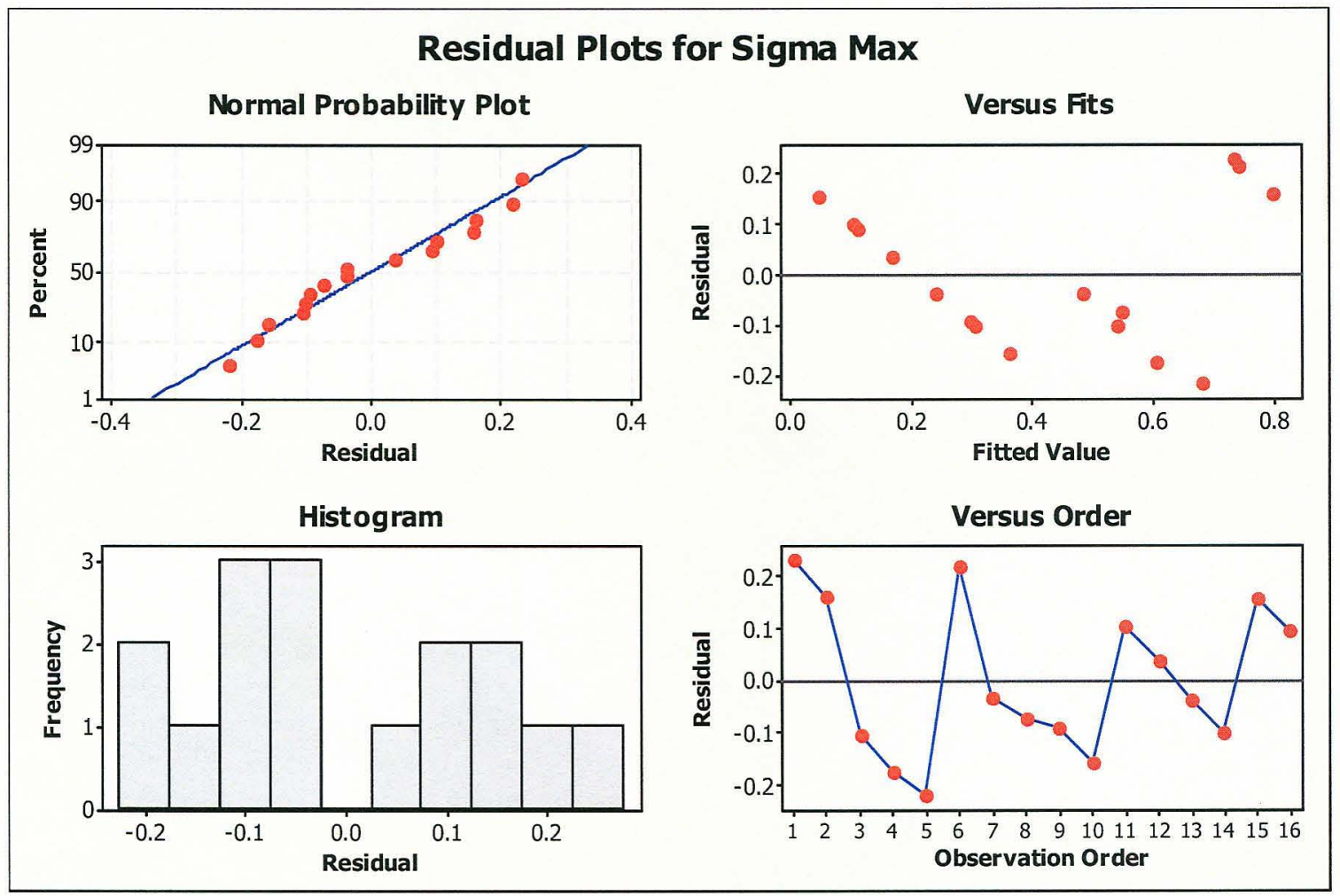


General Linear Model: Sigma Max versus Method, URL

$\begin{array}{llrl}\text { Factor } & \text { Type } & \text { Levels } & \text { Values } \\ \text { Method } & \text { fixed } & 2 & 0,1 \\ \text { URL } & \text { fixed } & 2 & 0.04,0.10\end{array}$

Analysis of Variance for Sigma Max, using Adjusted SS for Tests

$\begin{array}{lrrrrrr}\text { Source } & \text { DF } & \text { Seq SS } & \text { Adj SS } & \text { Adj MS } & \text { F } & \text { P } \\ \text { Method } & 1 & 0.76852 & 0.76852 & 0.76852 & 29.12 & 0.000 \\ \text { URI } & 1 & 0.15228 & 0.15228 & 0.15228 & 5.77 & 0.032 \\ \text { Error } & 13 & 0.34313 & 0.34313 & 0.02639 & & \\ \text { Total } & 15 & 1.26393 & & & & \end{array}$

$\mathrm{S}=0.162463 \quad \mathrm{R}-\mathrm{Sq}=72.85 \% \quad \mathrm{R}-\mathrm{Sq}(\operatorname{adj})=68.68 \%$

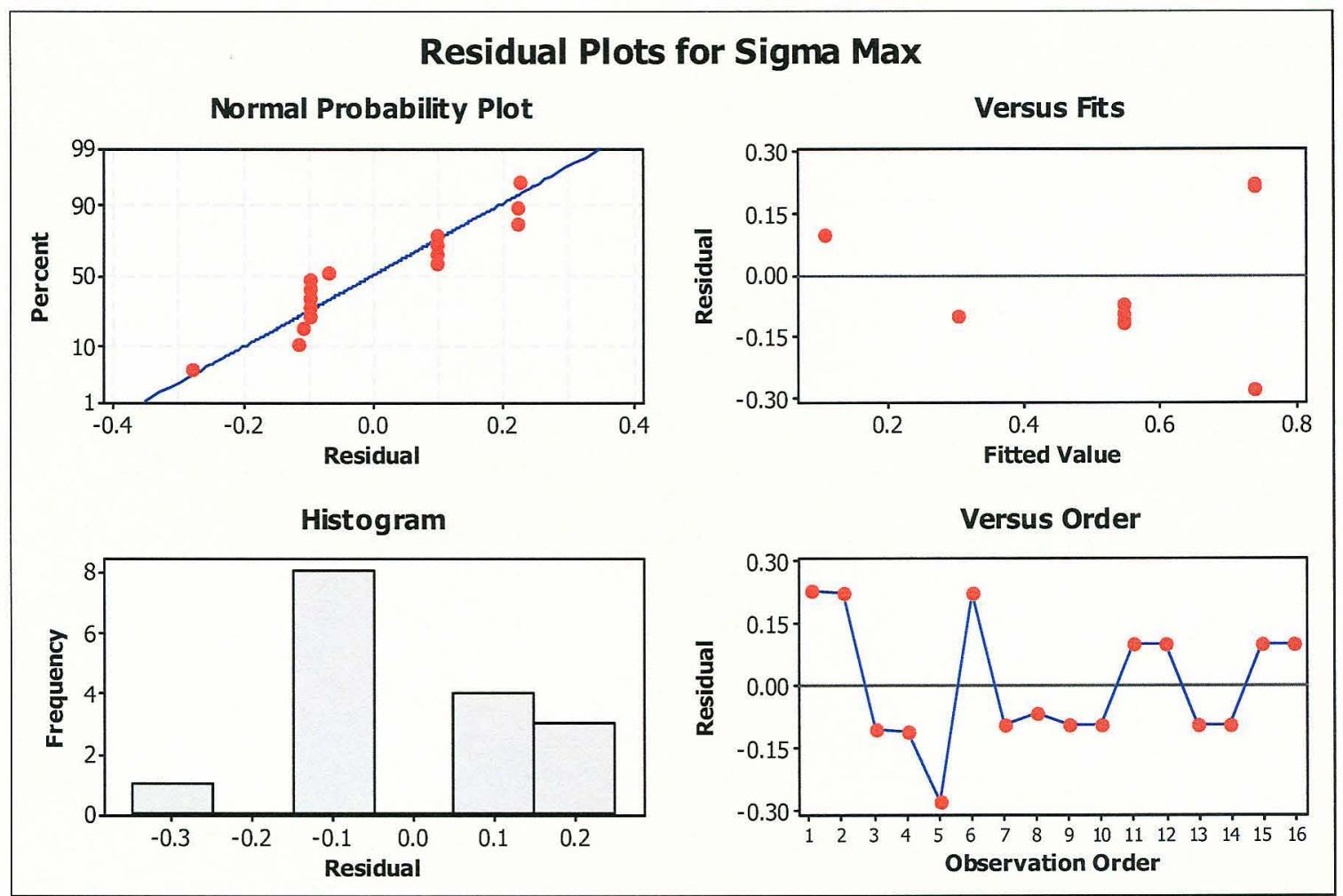


General Linear Model: Sigma Max versus Method, URL

$\begin{array}{llrl}\text { Factor } & \text { Type } & \text { Levels } & \text { Values } \\ \text { Method } & \text { fixed } & 2 & 0,1 \\ \text { URL } & \text { fixed } & 2 & 0.04,0.10\end{array}$

Analysis of Variance for Sigma Max, using Adjusted ss for Tests

$\begin{array}{lrrrrrr}\text { Source } & \text { DF } & \text { Seq SS } & \text { Adj SS } & \text { Adj MS } & \text { F } & \text { P } \\ \text { Method } & 1 & 0.76852 & 0.76852 & 0.76852 & 48.32 & 0.000 \\ \text { URL } & 1 & 0.15228 & 0.15228 & 0.15228 & 9.58 & 0.009 \\ \text { Method*URL } & 1 & 0.15228 & 0.15228 & 0.15228 & 9.58 & 0.009 \\ \text { Error } & 12 & 0.19084 & 0.19084 & 0.01590 & & \\ \text { Total } & 15 & 1.26393 & & & & \\ & & & & & & \\ \text { S }=0.126110 & \text { R-Sq }=84.90 \% & \text { R-Sq }(\text { adj })=81.13 \%\end{array}$

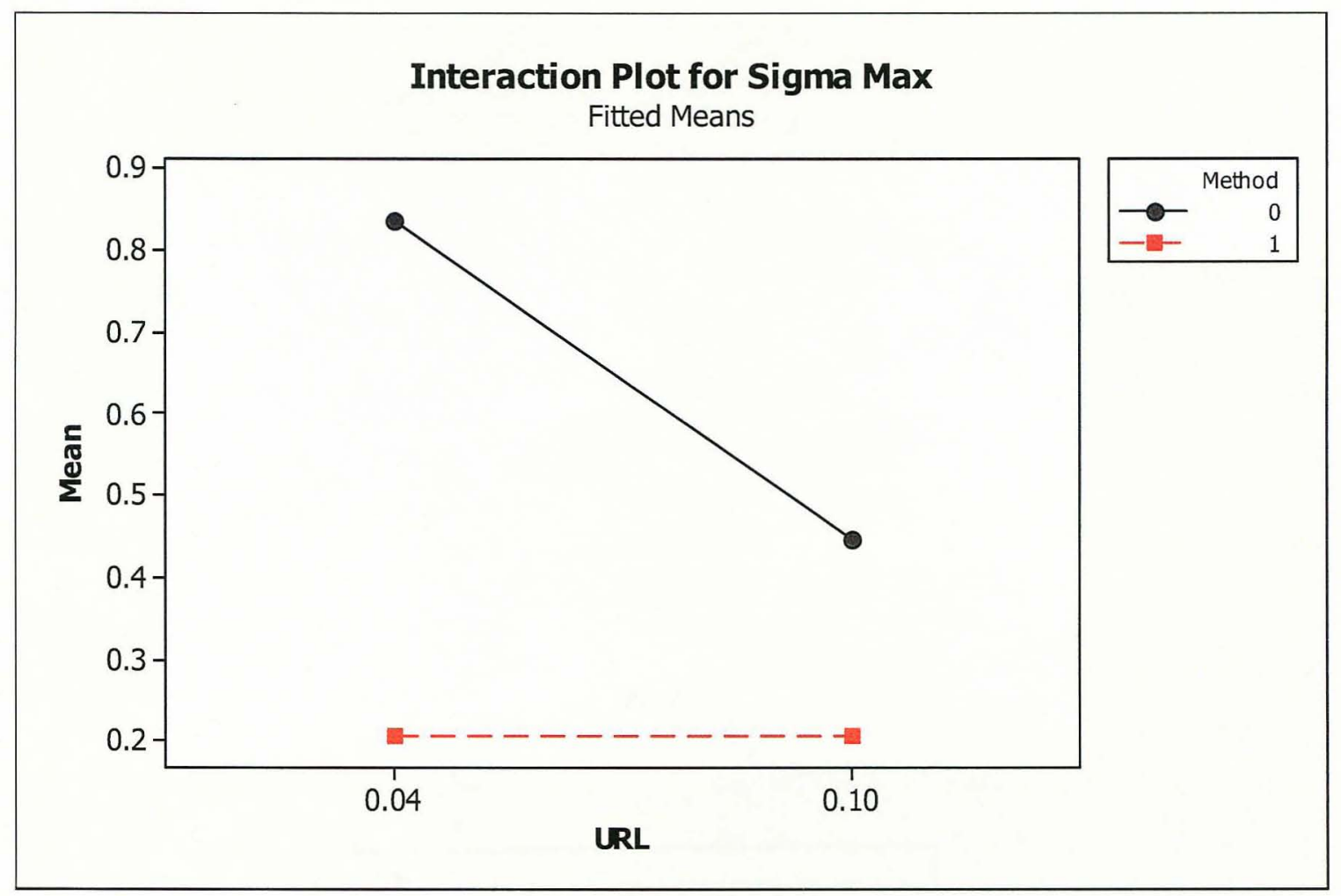



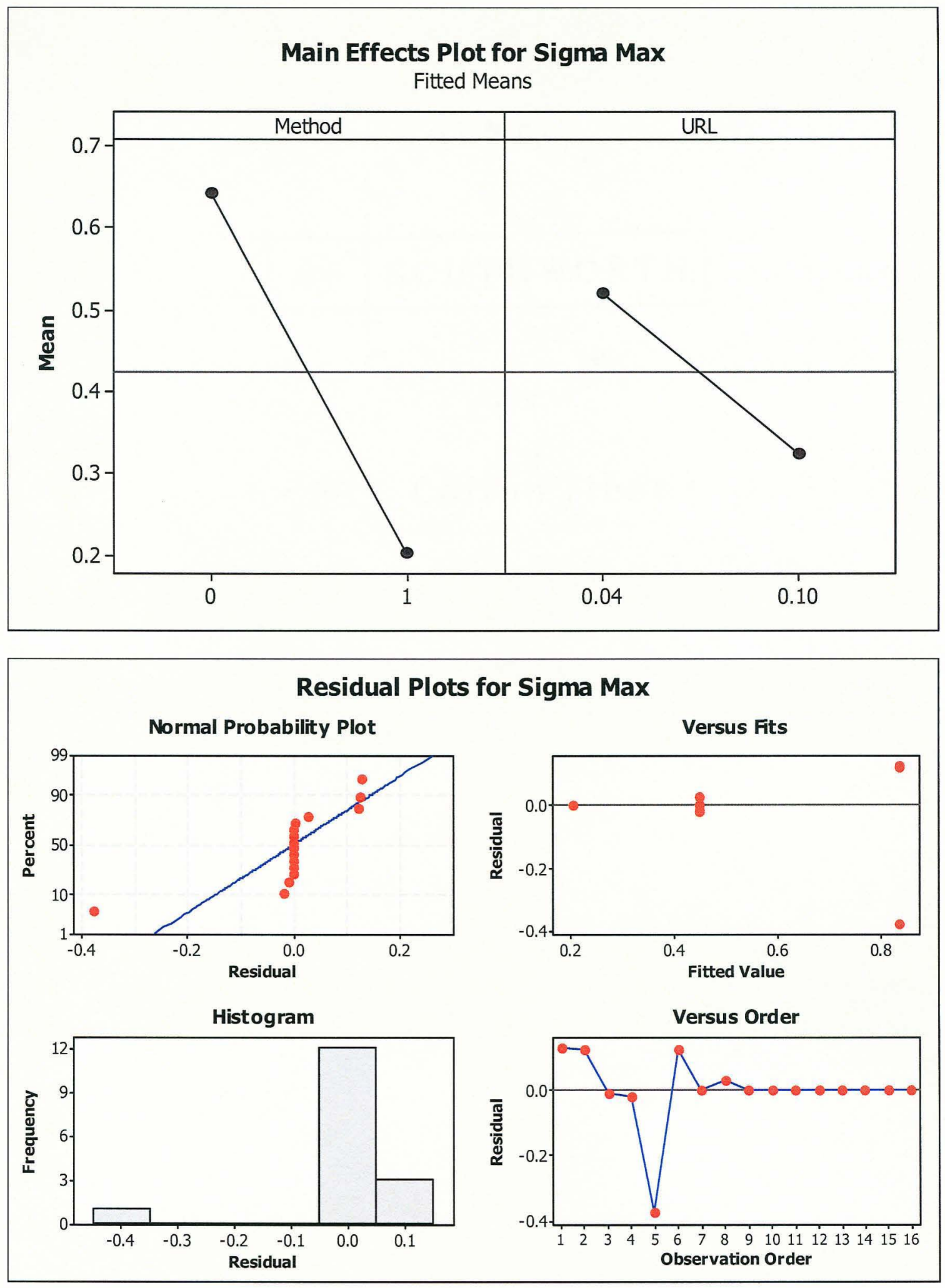


\section{Variance Average:}

\section{General Linear Model: Sigm Avg versus Method, Matching Method,}

-..

$\begin{array}{llrll}\text { Factor } & \text { Type } & \text { Levels } & \text { Values } \\ \text { Method } & \text { fixed } & 2 & 0,1 & \\ \text { Matching Method } & \text { fixed } & 2 & 0,1 & \\ \text { URL } & \text { fixed } & 2 & 0.04,0.10 \\ \text { Matching Sim } & \text { fixed } & 2 & 0.30,0.55\end{array}$

Analysis of Variance for Sigm Avg, using Adjusted SS for Tests

$\begin{array}{lrrrrrr}\text { Source } & \text { DF } & \text { Seq SS } & \text { Adj SS } & \text { Adj MS } & \text { F } & \text { P } \\ \text { Method } & 1 & 0.0252565 & 0.0252565 & 0.0252565 & 59.62 & 0.000 \\ \text { Matching Method } & 1 & 0.0021011 & 0.0021011 & 0.0021011 & 4.96 & 0.048 \\ \text { URL } & 1 & 0.0008100 & 0.0008100 & 0.0008100 & 1.91 & 0.194 \\ \text { Matching Sim } & 1 & 0.0003383 & 0.0003383 & 0.0003383 & 0.80 & 0.391 \\ \text { Error } & 11 & 0.0046596 & 0.0046596 & 0.0004236 & & \\ \text { Total } & 15 & 0.0331655 & & & \\ & & & & & \\ \text { S }=0.0205815 & \text { R-Sq }=85.95 \% & \text { R-Sq(adj) } & =80.84 \%\end{array}$

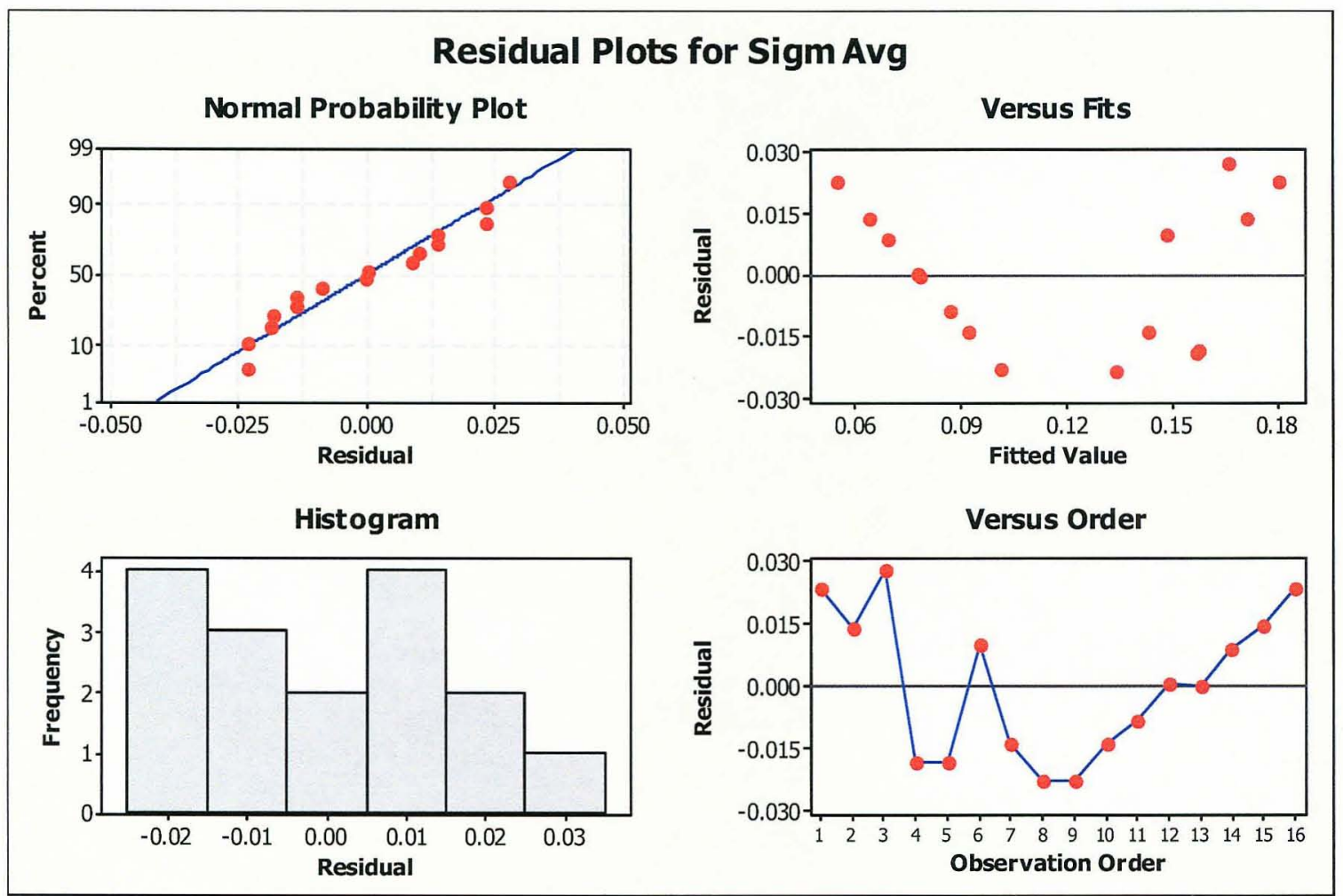


General Linear Model: Sigm Avg versus Method, Matching Method

$\begin{array}{llrl}\text { Factor } & \text { Type } & \text { Levels } & \text { Values } \\ \text { Method } & \text { fixed } & 2 & 0,1 \\ \text { Matching Method } & \text { fixed } & 2 & 0,1\end{array}$

Analysis of Variance for Sigm Avg, using Adjusted SS for Tests

\begin{tabular}{lrrrrrr} 
Source & DF & Seq SS & Adj SS & Adj MS & F & P \\
Method & 1 & 0.025256 & 0.025256 & 0.025256 & 56.53 & 0.000 \\
Matching Method & 1 & 0.002101 & 0.002101 & 0.002101 & 4.70 & 0.049 \\
Error & 13 & 0.005808 & 0.005808 & 0.000447 & & \\
Total & 15 & 0.033165 & & & \\
& & & & & \\
S $=0.0211368$ & R-Sq $=82.49 \%$ & R-Sq(adj) $=79.79 \%$ & \\
\hline
\end{tabular}

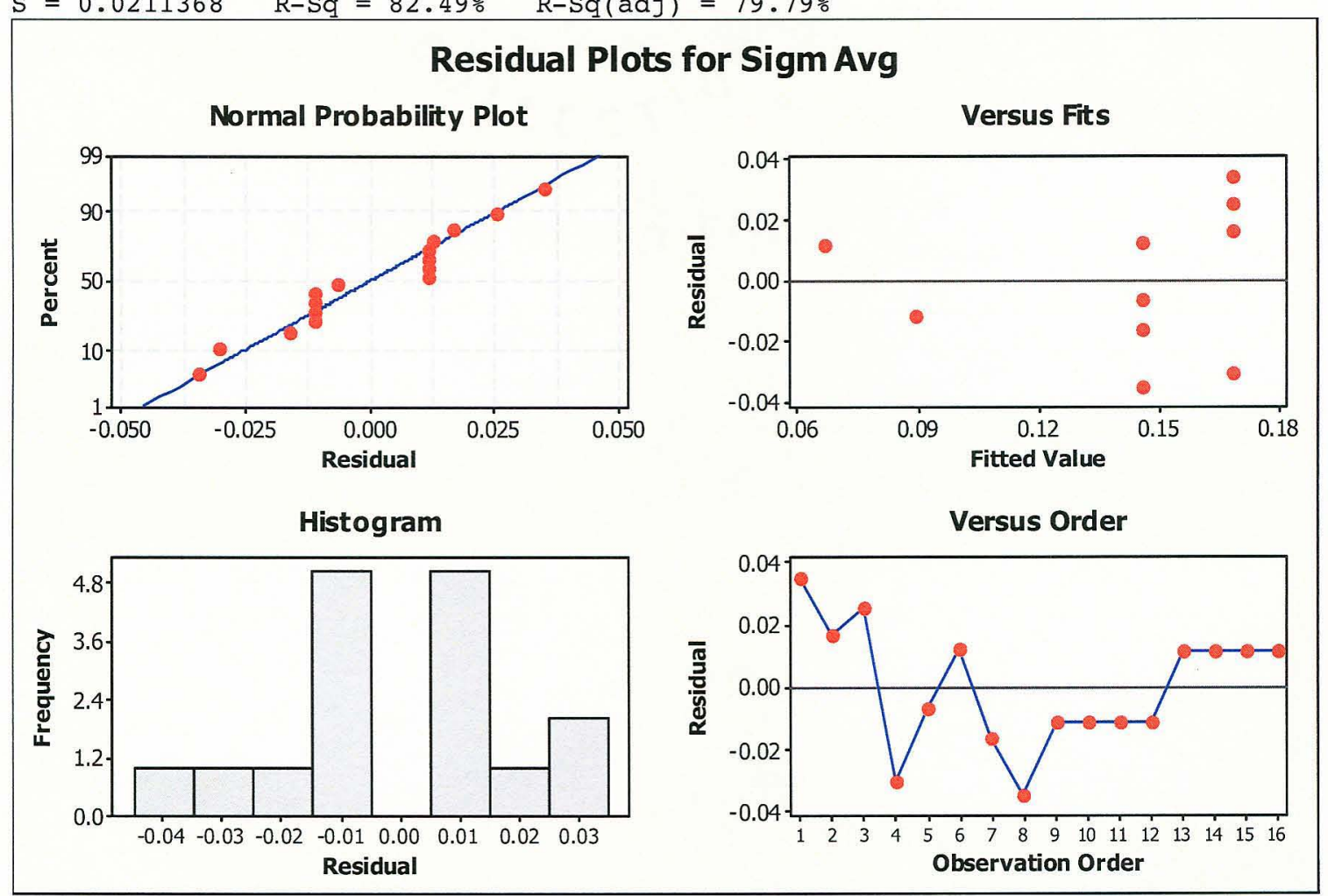

General Linear Model: Sigm Avg versus Method, Matching Method

$\begin{array}{llrl}\text { Factor } & \text { Type } & \text { Levels } & \text { Values } \\ \text { Method } & \text { fixed } & 2 & 0,1 \\ \text { Matching Method } & \text { fixed } & 2 & 0,1\end{array}$

Analysis of Variance for Sigm Avg, using Adjusted SS for Tests

$\begin{array}{lrrrrrr}\text { Source } & \text { DF } & \text { Seq SS } & \text { Adj SS } & \text { Adj MS } & \text { F } & \text { P } \\ \text { Method } & 1 & 0.0252565 & 0.0252565 & 0.0252565 & 81.76 & 0.000 \\ \text { Matching Method } & 1 & 0.0021011 & 0.0021011 & 0.0021011 & 6.80 & 0.023 \\ \text { Method*Matching Method } & 1 & 0.0021011 & 0.0021011 & 0.0021011 & 6.80 & 0.023 \\ \text { Error } & 12 & 0.0037069 & 0.0037069 & 0.0003089 & & \\ \text { Total } & 15 & 0.0331655 & & & & \end{array}$



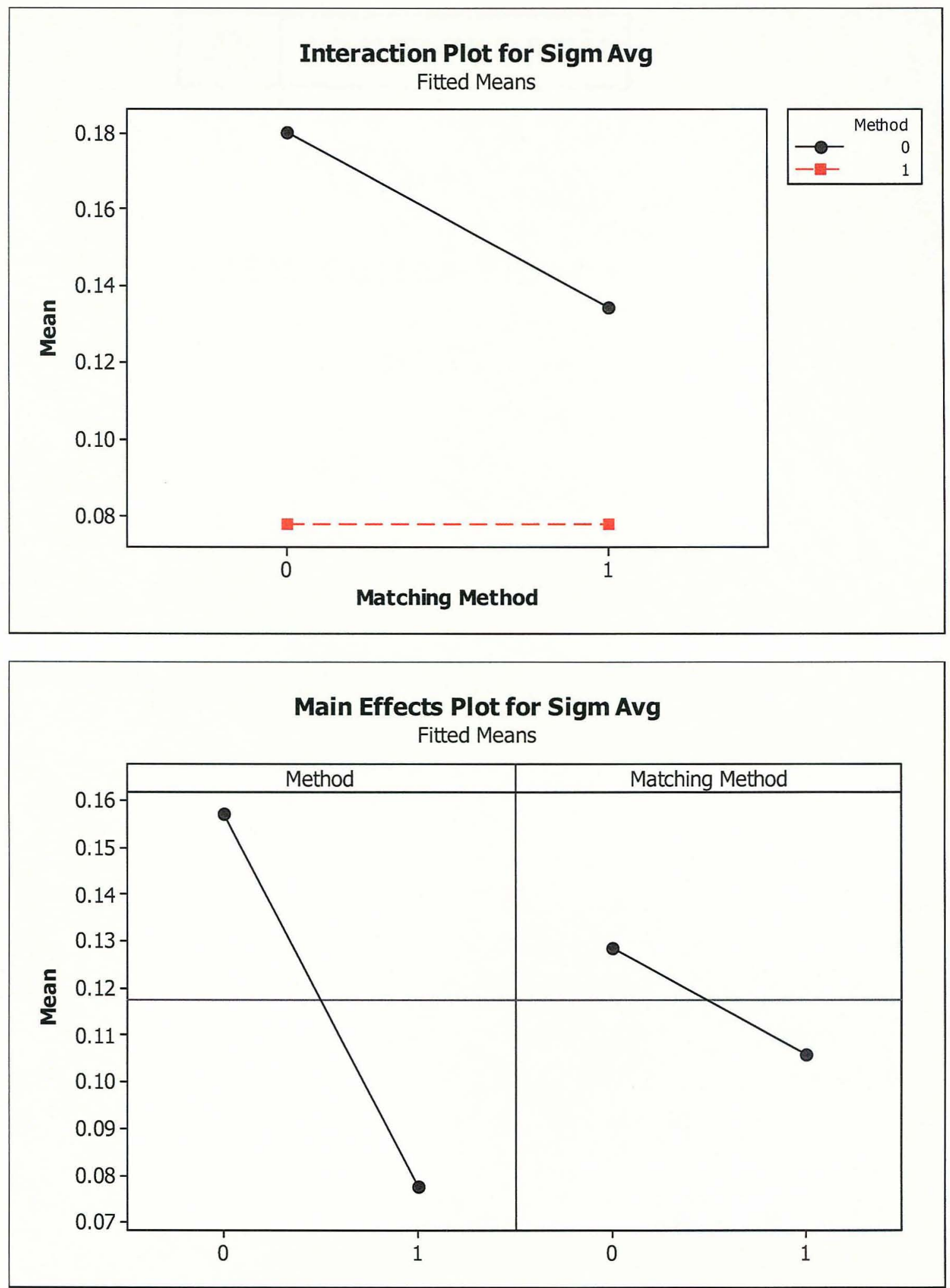


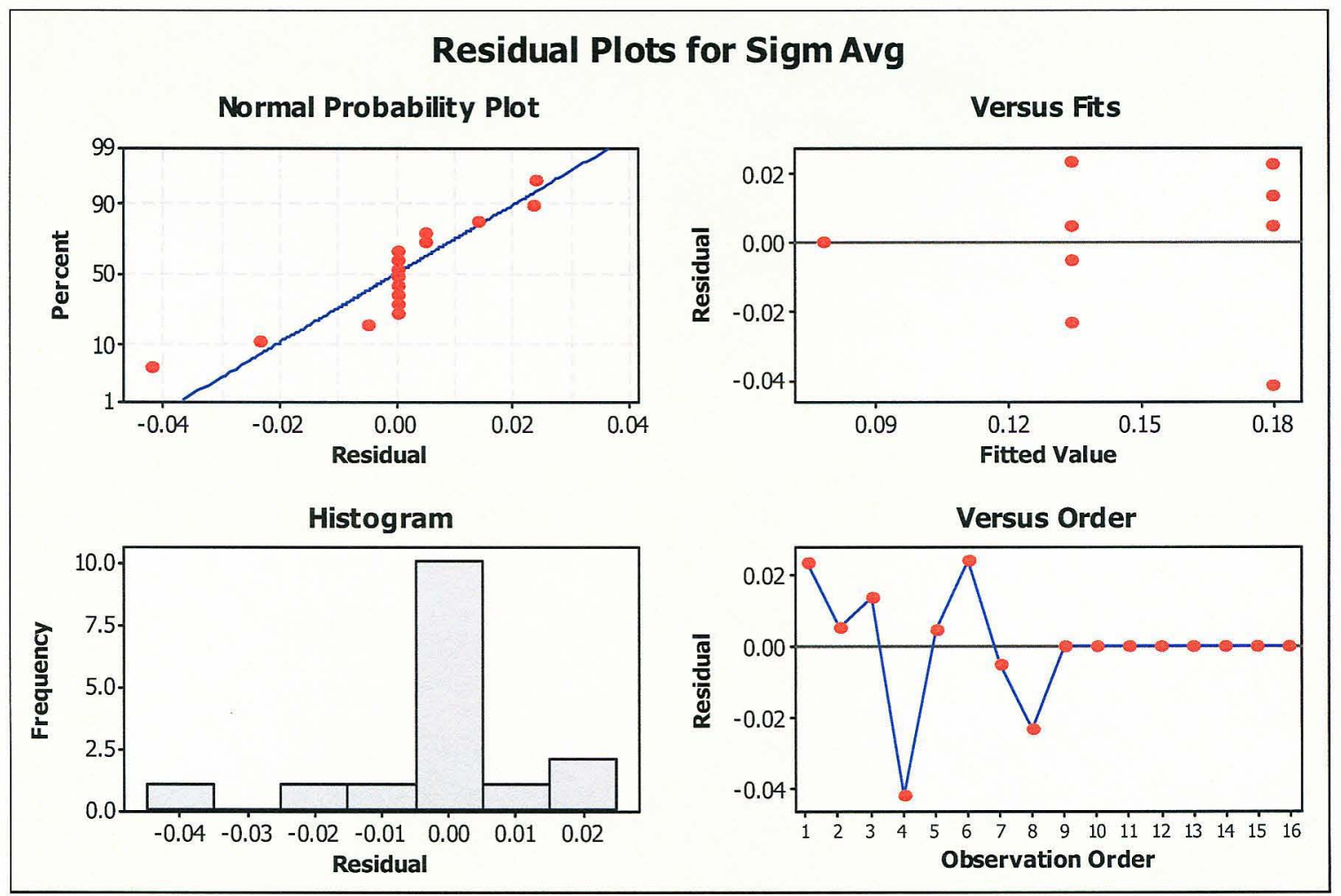

\section{Profiles Counts:}

General Linear Model: Prf Num versus Method, Matching Method, ...

$\begin{array}{llrl}\text { Factor } & \text { Type } & \text { Levels } & \text { Values } \\ \text { Method } & \text { fixed } & 2 & 0,1 \\ \text { Matching Method } & \text { fixed } & 2 & 0,1 \\ \text { URL } & \text { fixed } & 2 & 0.04,0.10 \\ \text { Matching Sim } & \text { fixed } & 2 & 0.30,0.55\end{array}$

Analysis of Variance for Prf Num, using Adjusted SS for Tests

$\begin{array}{lrrrrrr}\text { Source } & \text { DF } & \text { Seq SS } & \text { Adj SS } & \text { Adj MS } & \text { F } & \text { P } \\ \text { Method } & 1 & 1785.06 & 1785.06 & 1785.06 & 30.39 & 0.000 \\ \text { Matching Method } & 1 & 95.06 & 95.06 & 95.06 & 1.62 & 0.230 \\ \text { URL } & 1 & 14.06 & 14.06 & 14.06 & 0.24 & 0.634 \\ \text { Matching Sim } & 1 & 217.56 & 217.56 & 217.56 & 3.70 & 0.081 \\ \text { Error } & 11 & 646.19 & 646.19 & 58.74 & & \\ \text { Total } & 15 & 2757.94 & & & & \end{array}$

$\mathrm{S}=7.66448 \quad \mathrm{R}-\mathrm{Sq}=76.57 \% \quad \mathrm{R}-\mathrm{Sq}(\operatorname{adj})=68.05 \%$ 


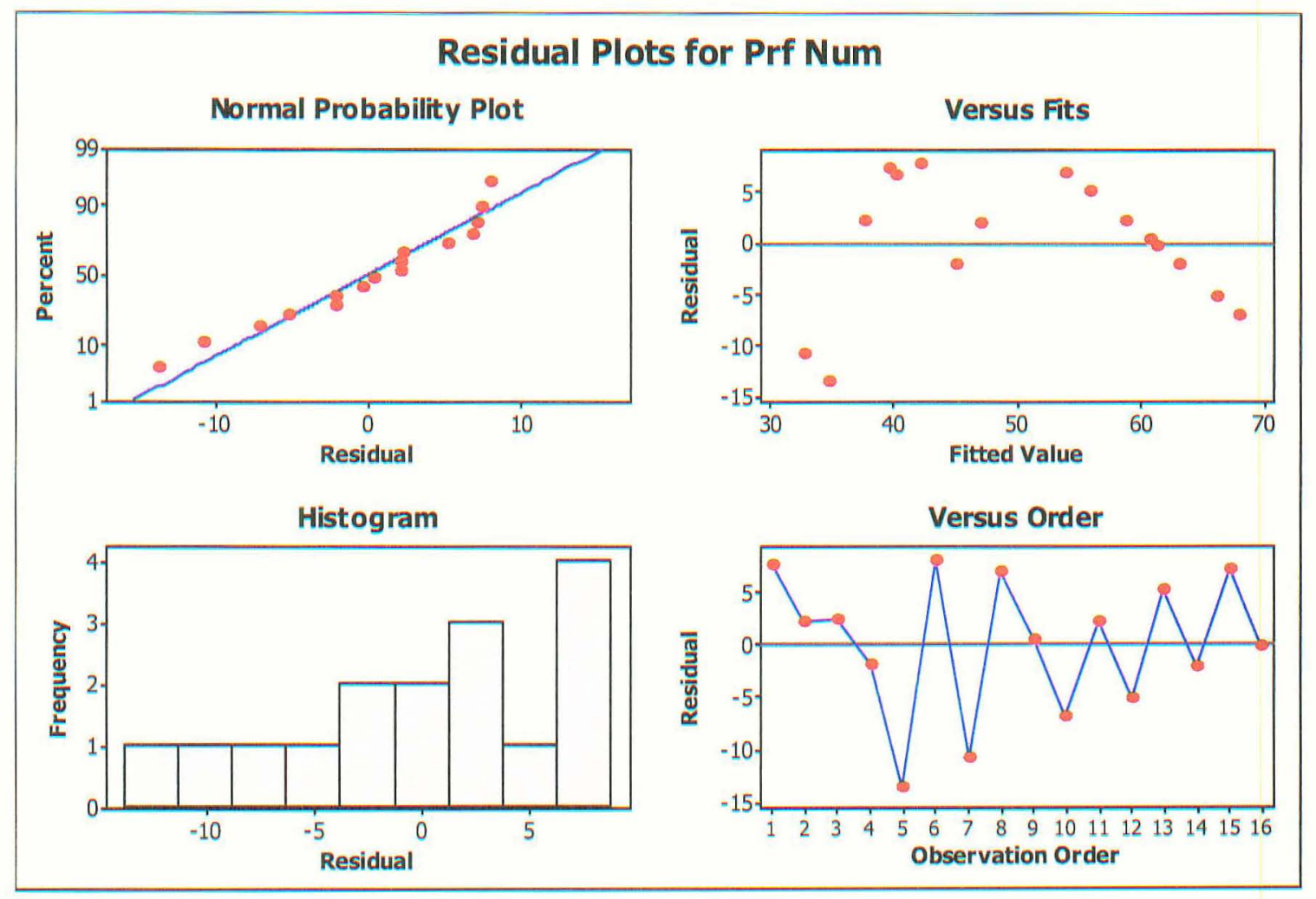

General Linear Model: Prf Num versus Method, Matching Sim

$\begin{array}{llrl}\text { Factor } & \text { Type } & \text { Levels } & \text { Values } \\ \text { Method } & \text { fixed } & 2 & 0,1 \\ \text { Matching Sim } & \text { fixed } & 2 & 0.30,0.55\end{array}$

Analysis of Variance for Prf Num, using Adjusted SS for Tests

$\begin{array}{lrrrrrr}\text { Source } & \text { DF } & \text { Seq SS } & \text { Adj SS } & \text { Adj MS } & \text { F } & \text { P } \\ \text { Method } & 1 & 1785.1 & 1785.1 & 1785.1 & 30.72 & 0.000 \\ \text { Matching Sim } & 1 & 217.6 & 217.6 & 217.6 & 3.74 & 0.075 \\ \text { Error } & 13 & 755.3 & 755.3 & 58.1 & & \\ \text { Total } & 15 & 2757.9 & & & & \\ & \\ \text { S }=7.62240 & \text { R-Sq }=72.61 \% & \text { R-Sq }(\text { adj })=68.40 \%\end{array}$




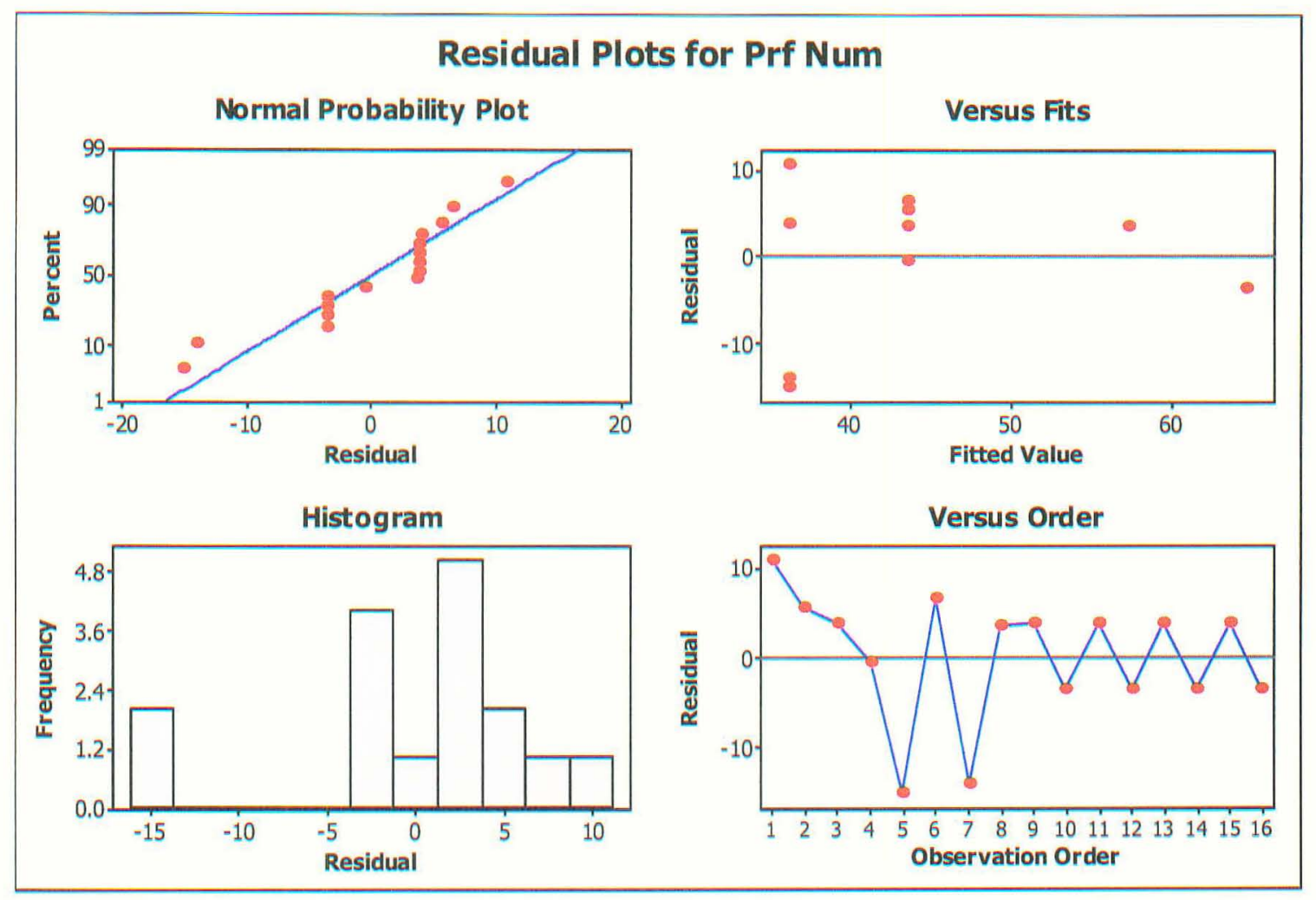

General Linear Model: Prf Num versus Method, Matching Sim

$\begin{array}{llrl}\text { Factor } & \text { Type } & \text { Levels } & \text { Values } \\ \text { Method } & \text { fixed } & 2 & 0,1 \\ \text { Matching Sim } & \text { fixed } & 2 & 0.30,0.55\end{array}$

Analysis of Variance for Prf Num, using Adjusted ss for Tests

$\begin{array}{lrrrrrr}\text { Source } & \text { DF } & \text { Seq SS } & \text { Adj SS } & \text { Adj MS } & \text { F } & \text { P } \\ \text { Method } & 1 & 1785.06 & 1785.06 & 1785.06 & 39.83 & 0.000 \\ \text { Matching Sim } & 1 & 217.56 & 217.56 & 217.56 & 4.85 & 0.048 \\ \text { Method*Matching Sim } & 1 & 217.56 & 217.56 & 217.56 & 4.85 & 0.048 \\ \text { Error } & 12 & 537.75 & 537.75 & 44.81 & & \\ \text { Total } & 15 & 2757.94 & & & \\ & \\ \text { S }=6.69421 \quad \text { R-Sq }=80.50 \% \quad \text { R-Sq (adj) }=75.63 \%\end{array}$



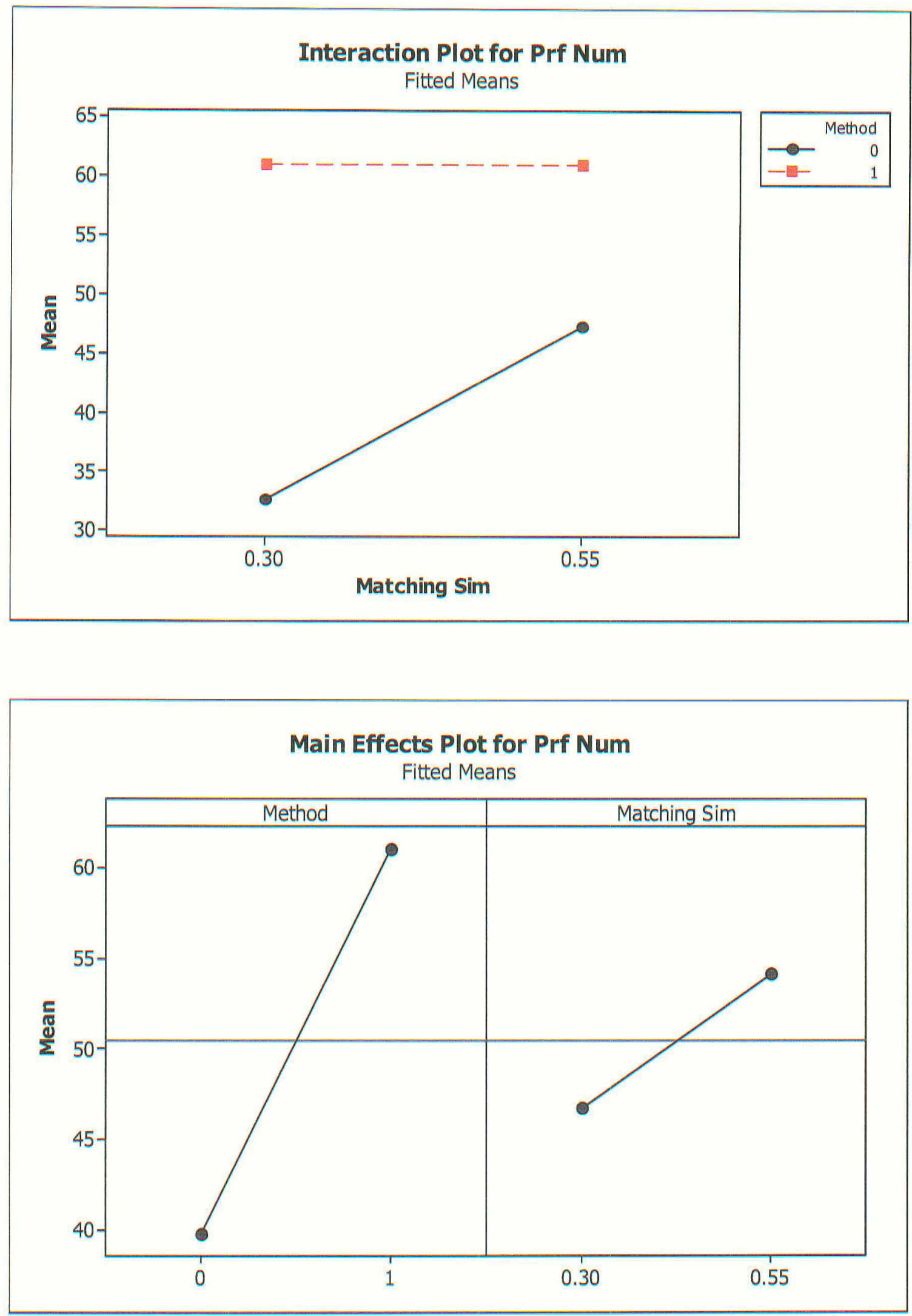


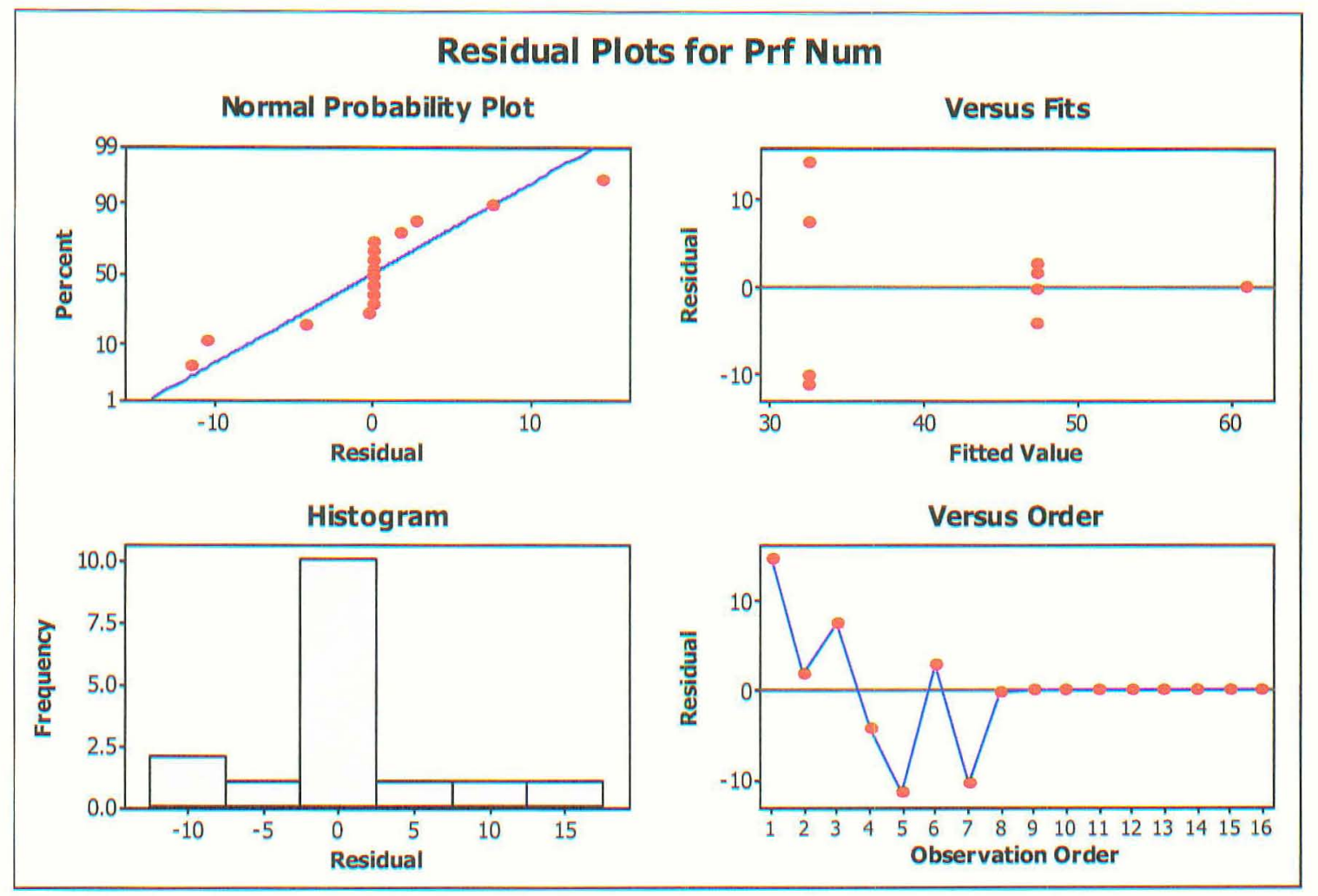

Cardinality of profile with maximum sigma:

General Linear Model: Card versus Method, Matching Method, ...

$\begin{array}{llrl}\text { Factor } & \text { Type } & \text { Levels } & \text { Values } \\ \text { Method } & \text { fixed } & 2 & 0,1 \\ \text { Matching Method } & \text { fixed } & 2 & 0,1 \\ \text { URL } & \text { fixed } & 2 & 0.04,0.10 \\ \text { Matching Sim } & \text { fixed } & 2 & 0.30,0.55\end{array}$

Analysis of Variance for Card, using Adjusted SS for Tests

$\begin{array}{lrrrrrr}\text { Source } & \text { DF } & \text { Seq SS } & \text { Adj SS } & \text { Adj MS } & \text { F } & \text { P } \\ \text { Method } & 1 & 1221.3 & 1221.3 & 1221.3 & 4.06 & 0.069 \\ \text { Matching Method } & 1 & 808.3 & 808.3 & 808.3 & 2.68 & 0.130 \\ \text { URL } & 1 & 17.4 & 17.4 & 17.4 & 0.06 & 0.814 \\ \text { Matching Sim } & 1 & 806.8 & 806.8 & 806.8 & 2.68 & 0.130 \\ \text { Error } & 11 & 3312.5 & 3312.5 & 301.1 & & \\ \text { Total } & 15 & 6166.4 & & & & \end{array}$

$\mathrm{S}=17.3532 \quad \mathrm{R}-\mathrm{Sq}=46.28 \% \quad \mathrm{R}-\mathrm{Sq}(\mathrm{adj})=26.75 \%$ 


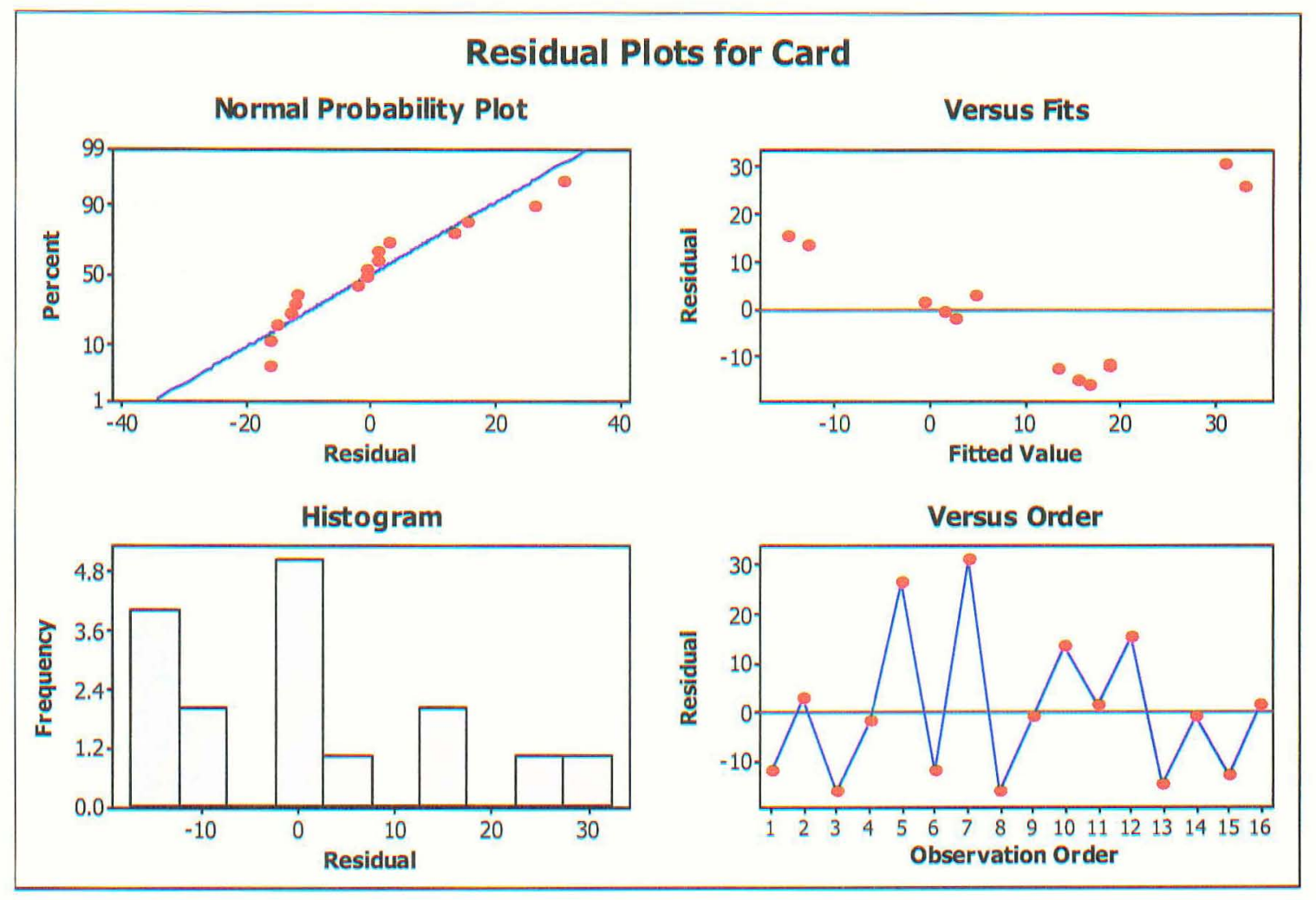

General Linear Model: Card versus Method, Matching Method, Matching Sim

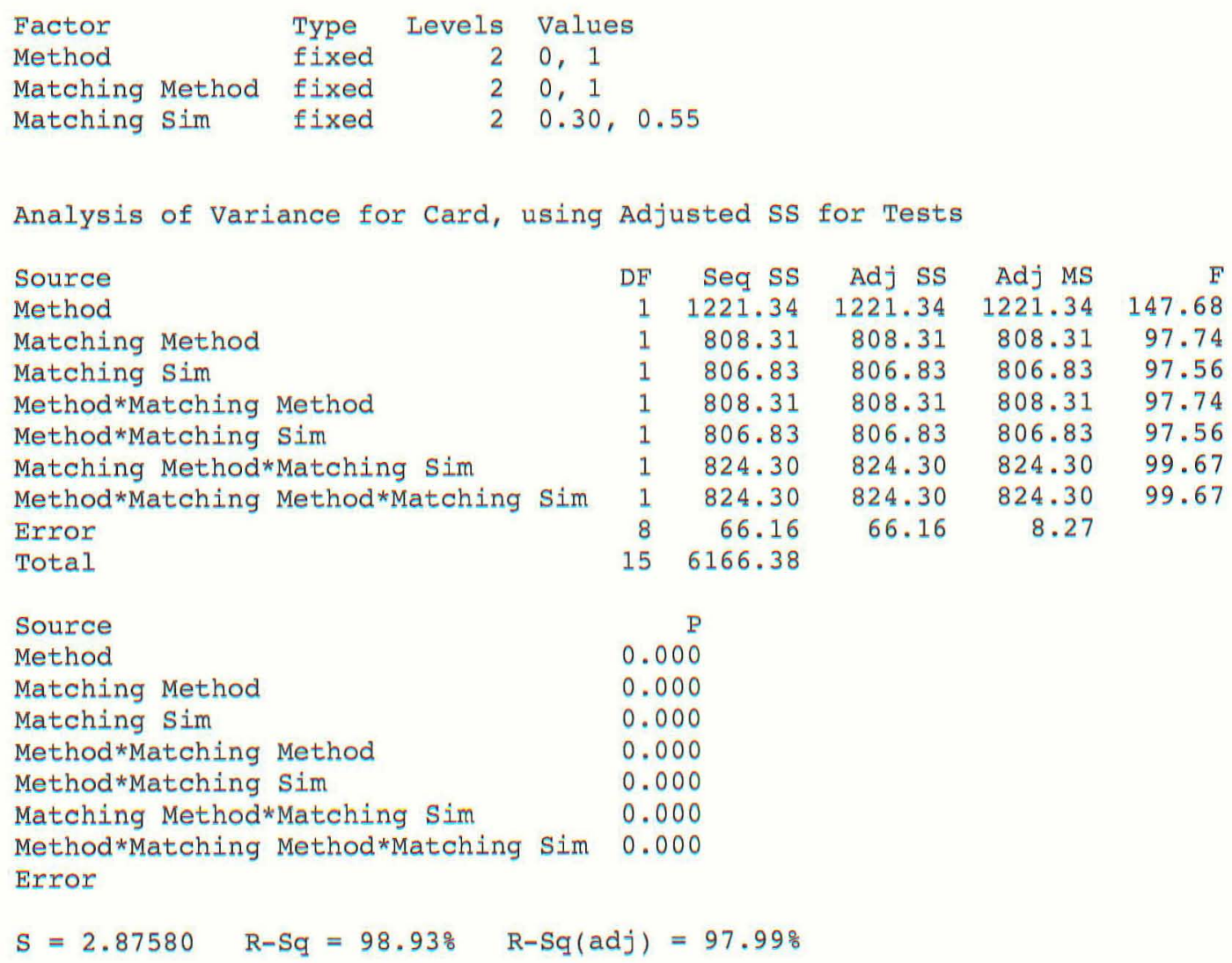




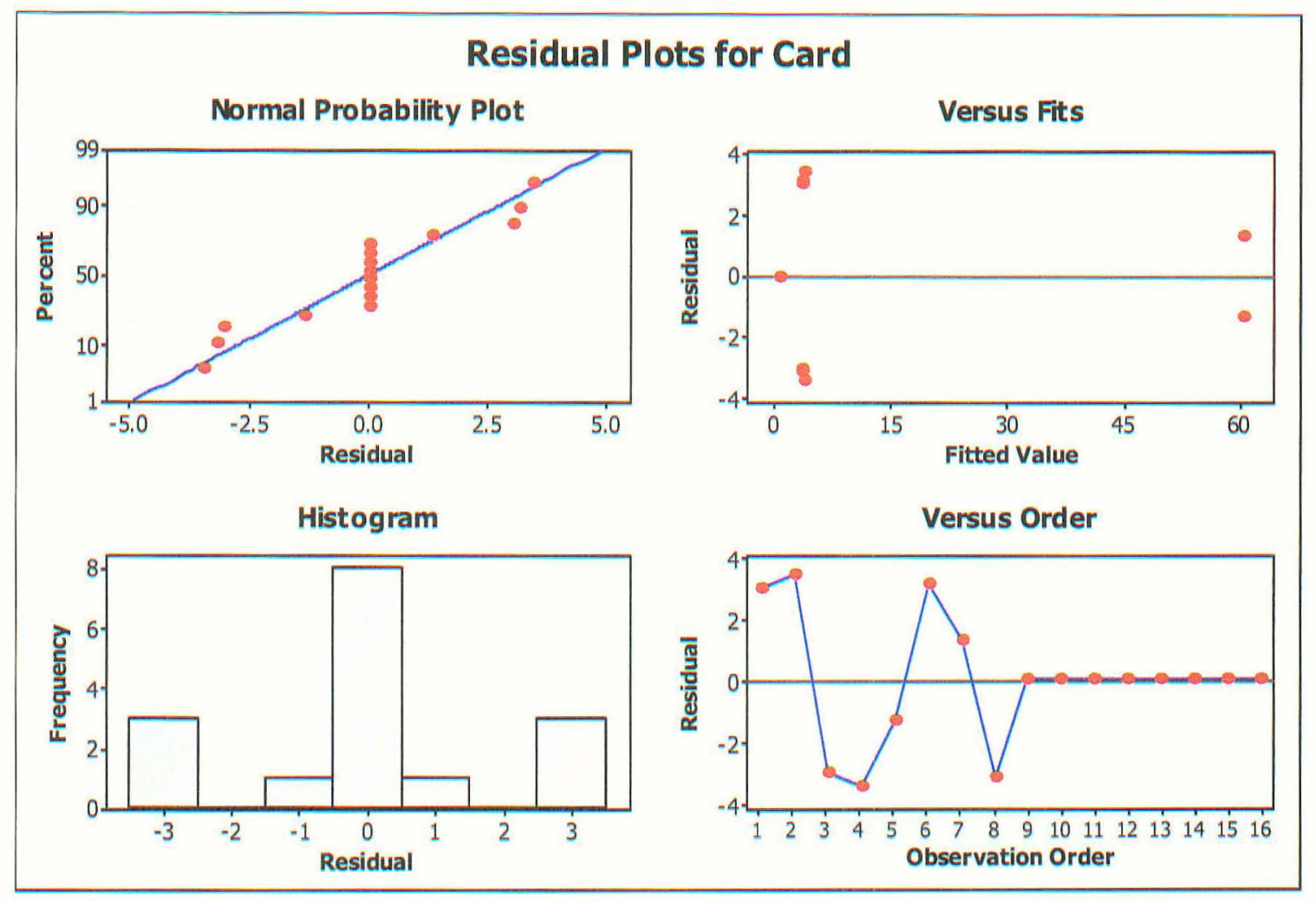

\section{Number of duplicate profiles:}

General Linear Model: Duplicate versus Method, Matching Method, ...

$\begin{array}{llrll}\text { Factor } & \text { Type } & \text { Levels } & \text { Values } \\ \text { Method } & \text { fixed } & 2 & 0,1 & \\ \text { Matching Method } & \text { fixed } & 2 & 0,1 & \\ \text { URL } & \text { fixed } & 2 & 0.04,0.10 \\ \text { Matching Sim } & \text { fixed } & 2 & 0.30,0.55\end{array}$

Analysis of Variance for Duplicate, using Adjusted ss for Tests

$\begin{array}{lrrrrrr}\text { Source } & \text { DF } & \text { Seq SS } & \text { Adj SS } & \text { Adj MS } & \text { F } & \text { P } \\ \text { Method } & 1 & 0.0008105 & 0.0008105 & 0.0008105 & 7.44 & 0.020 \\ \text { Matching Method } & 1 & 0.0002711 & 0.0002711 & 0.0002711 & 2.49 & 0.143 \\ \text { URL } & 1 & 0.0000669 & 0.0000669 & 0.0000669 & 0.61 & 0.450 \\ \text { Matching Sim } & 1 & 0.0003665 & 0.0003665 & 0.0003665 & 3.36 & 0.094 \\ \text { Error } & 11 & 0.0011990 & 0.0011990 & 0.0001090 & & \\ \text { Total } & 15 & 0.0027140 & & & & \\ & & & & & \\ \text { S }=0.0104405 & \text { R-Sq }=55.82 \% & \text { R-Sq(adj) }=39.76 \%\end{array}$




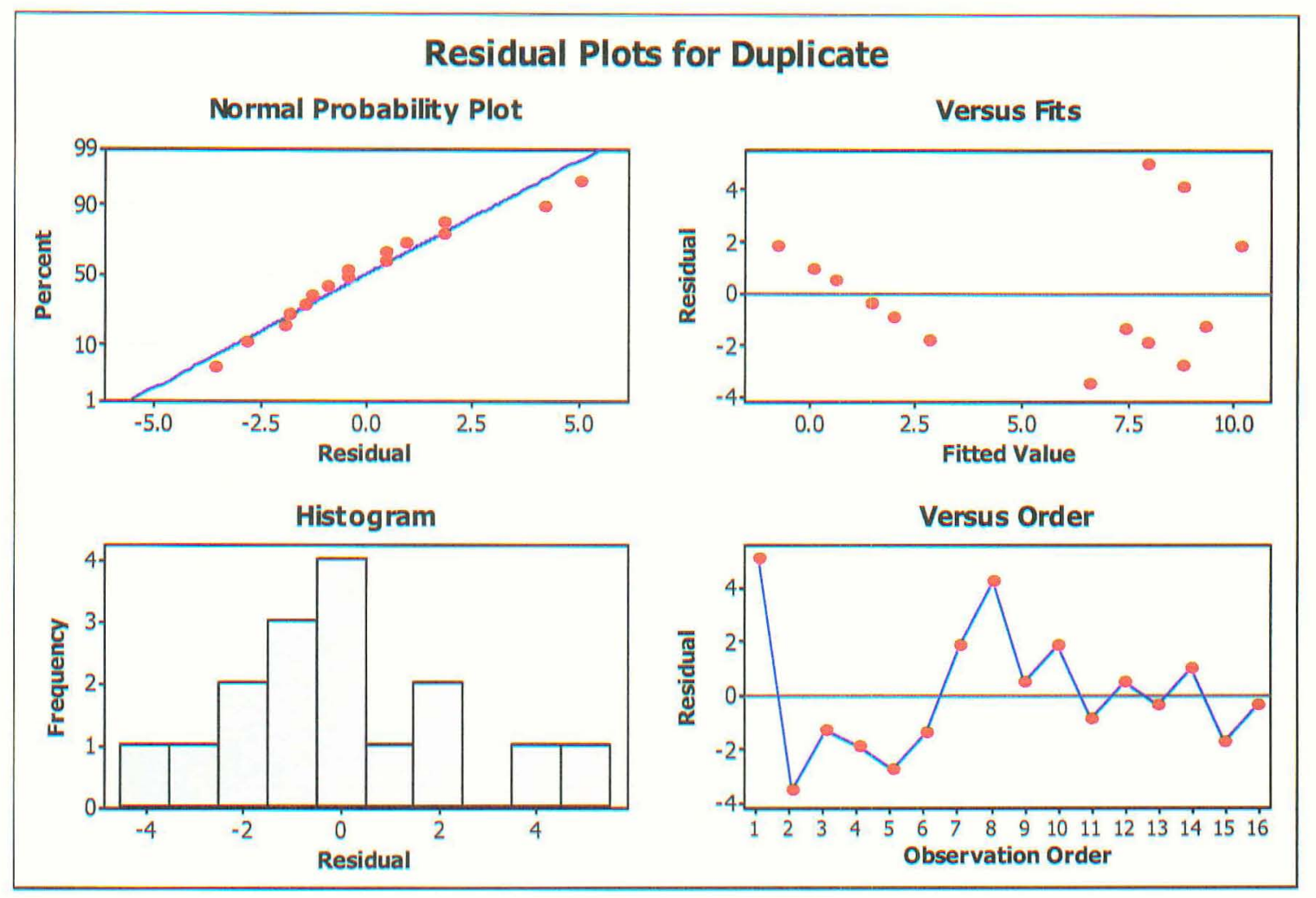

General Linear Model: Duplicate versus Method, Matching Method, ...

$\begin{array}{llrl}\text { Factor } & \text { Type } & \text { Levels } & \text { Values } \\ \text { Method } & \text { fixed } & 2 & 0,1 \\ \text { Matching Method } & \text { fixed } & 2 & 0,1 \\ \text { URL } & \text { fixed } & 2 & 0.04,0.10 \\ \text { Matching Sim } & \text { fixed } & 2 & 0.30,0.55\end{array}$

Analysis of Variance for Duplicate, using Adjusted SS for Tests

$\begin{array}{lrrrrrr}\text { Source } & \text { DF } & \text { Seq SS } & \text { Adj SS } & \text { Adj MS } & \text { F } & \text { P } \\ \text { Method } & 1 & 0.0008105 & 0.0008105 & 0.0008105 & * * & \\ \text { Matching Method } & 1 & 0.0002711 & 0.0002711 & 0.0002711 & * * \\ \text { URL } & 1 & 0.0000669 & 0.0000669 & 0.0000669 & * * \\ \text { Matching Sim } & 1 & 0.0003665 & 0.0003665 & 0.0003665 & * * \\ \text { Method*Matching Method } & 1 & 0.0002711 & 0.0002711 & 0.0002711 & * * \\ \text { Method*URL } & 1 & 0.0000669 & 0.0000669 & 0.0000669 & * * \\ \text { Method*Matching Sim } & 1 & 0.0003665 & 0.0003665 & 0.0003665 & * * \\ \text { Matching Method*URL } & 1 & 0.0000500 & 0.0000500 & 0.0000500 & * * \\ \text { Matching Method*Matching Sim } & 1 & 0.0001601 & 0.0001601 & 0.0001601 & * * \\ \text { URL*Matching Sim } & 1 & 0.0000069 & 0.0000069 & 0.0000069 & * * \\ \text { Method*Matching Method*URL } & 1 & 0.0000500 & 0.0000500 & 0.0000500 & * * \\ \text { Method*Matching Method*Matching Sim } & 1 & 0.0001601 & 0.0001601 & 0.0001601 & * * \\ \text { Method*URL*Matching Sim } & 1 & 0.0000069 & 0.0000069 & 0.0000069 & * * \\ \text { Matching Method*URL*Matching Sim } & 1 & 0.0000303 & 0.0000303 & 0.0000303 & * * \\ \text { Method*Matching Method*URL* } & 1 & 0.0000303 & 0.0000303 & 0.0000303 & * * \\ \quad \text { Matching Sim } & & & & & & \\ \text { Error } & 0 & * & & * & & \\ \text { Total } & 15 & 0.0027140 & & & \end{array}$




\section{Profiles Density :}

General Linear Model: Density versus Method, Matching Method, ...

$\begin{array}{llrl}\text { Factor } & \text { Type } & \text { Levels } & \text { Values } \\ \text { Method } & \text { fixed } & 2 & 0,1 \\ \text { Matching Method } & \text { fixed } & 2 & 0,1 \\ \text { URL } & \text { fixed } & 2 & 0.04,0.10 \\ \text { Matching Sim } & \text { fixed } & 2 & 0.30,0.55\end{array}$

Analysis of Variance for Density, using Adjusted SS for Tests

$\begin{array}{lrrrrrr}\text { Source } & \text { DF } & \text { Seq SS } & \text { Adj SS } & \text { Adj MS } & \text { F } & \text { P } \\ \text { Method } & 1 & 25658023 & 25658023 & 25658023 & 8.35 & 0.015 \\ \text { Matching Method } & 1 & 19797772 & 19797772 & 19797772 & 6.44 & 0.028 \\ \text { URL } & 1 & 232358 & 232358 & 232358 & 0.08 & 0.788 \\ \text { Matching Sim } & 1 & 6894093 & 6894093 & 6894093 & 2.24 & 0.162 \\ \begin{array}{l}\text { Error } \\ \text { Total }\end{array} & 11 & 33810800 & 33810800 & 3073709 & & \\ & 15 & 86393045 & & & \\ \text { S }=1753.20 & \text { R-Sq }=60.86 \% & \text { R-Sq }(\operatorname{adj})=46.63 \%\end{array}$

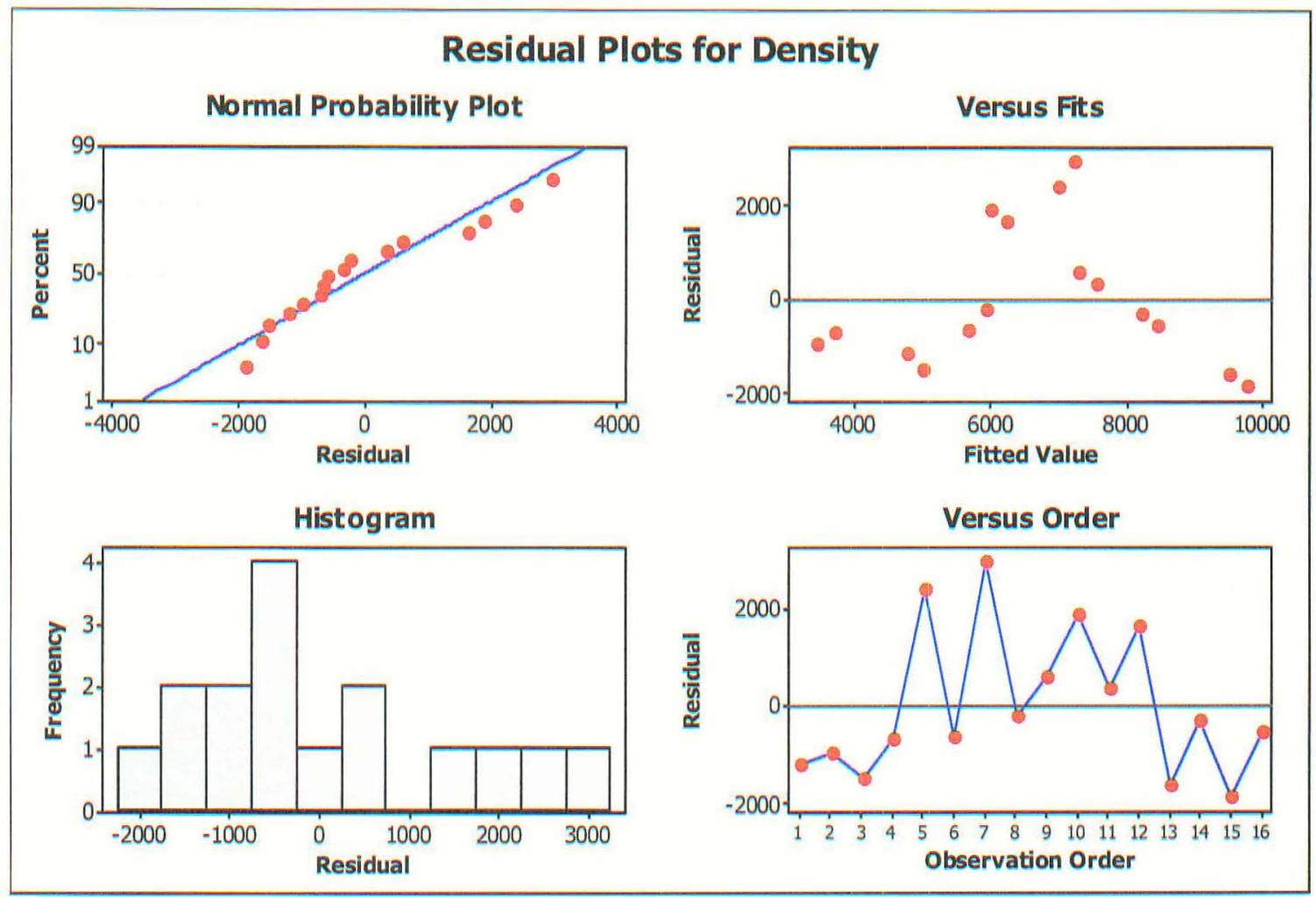


General Linear Model: Density versus Method, Matching Method

$\begin{array}{llrl}\text { Factor } & \text { Type } & \text { Levels } & \text { Values } \\ \text { Method } & \text { fixed } & 2 & 0,1 \\ \text { Matching Method } & \text { fixed } & 2 & 0,1\end{array}$

Analysis of Variance for Density, using Adjusted ss for Tests

$\begin{array}{lrrrrrr}\text { Source } & \text { DF } & \text { Seq SS } & \text { Adj SS } & \text { Adj MS } & \text { F } & \text { P } \\ \text { Method } & 1 & 25658023 & 25658023 & 25658023 & 8.15 & 0.014 \\ \text { Matching Method } & 1 & 19797772 & 19797772 & 19797772 & 6.29 & 0.026 \\ \begin{array}{l}\text { Error } \\ \text { Total }\end{array} & 13 & 40937251 & 40937251 & 3149019 & & \\ & 15 & 86393045 & & & & \\ \text { S }=1774.55 \quad \text { R-Sq }=52.62 \% & \text { R-Sq }(\operatorname{adj})=45.33 \% & & \end{array}$

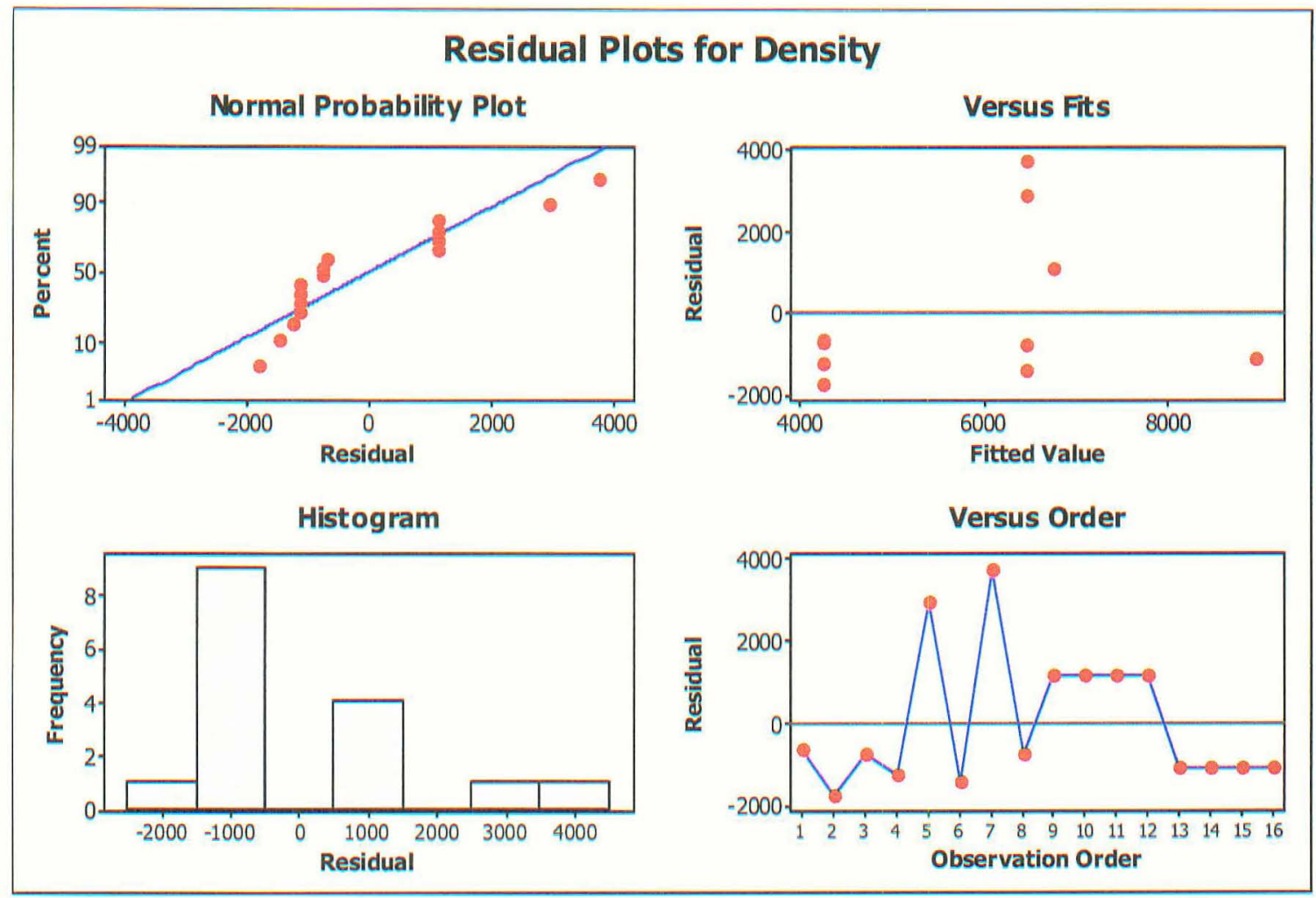


General Linear Model: Density versus Method, Matching Method

$\begin{array}{llrl}\text { Factor } & \text { Type } & \text { Levels } & \text { Values } \\ \text { Method } & \text { fixed } & 2 & 0,1 \\ \text { Matching Method } & \text { fixed } & 2 & 0,1\end{array}$

Analysis of Variance for Density, using Adjusted SS for Tests

$\begin{array}{lrrrrrr}\text { Source } & \text { DF } & \text { Seq SS } & \text { Adj SS } & \text { Adj MS } & \text { F } & \text { P } \\ \text { Method } & 1 & 25658023 & 25658023 & 25658023 & 14.56 & 0.002 \\ \text { Matching Method } & 1 & 19797772 & 19797772 & 19797772 & 11.24 & 0.006 \\ \text { Method*Matching Method } & 1 & 19797772 & 19797772 & 19797772 & 11.24 & 0.006 \\ \text { Error } & 12 & 21139479 & 21139479 & 1761623 & & \\ \text { Total } & 15 & 86393045 & & & & \\ & & & & & & \\ \text { S }=1327.26 \quad \text { R-Sq }=75.53 \% & \text { R-Sq }(\text { adj })=69.41 \%\end{array}$

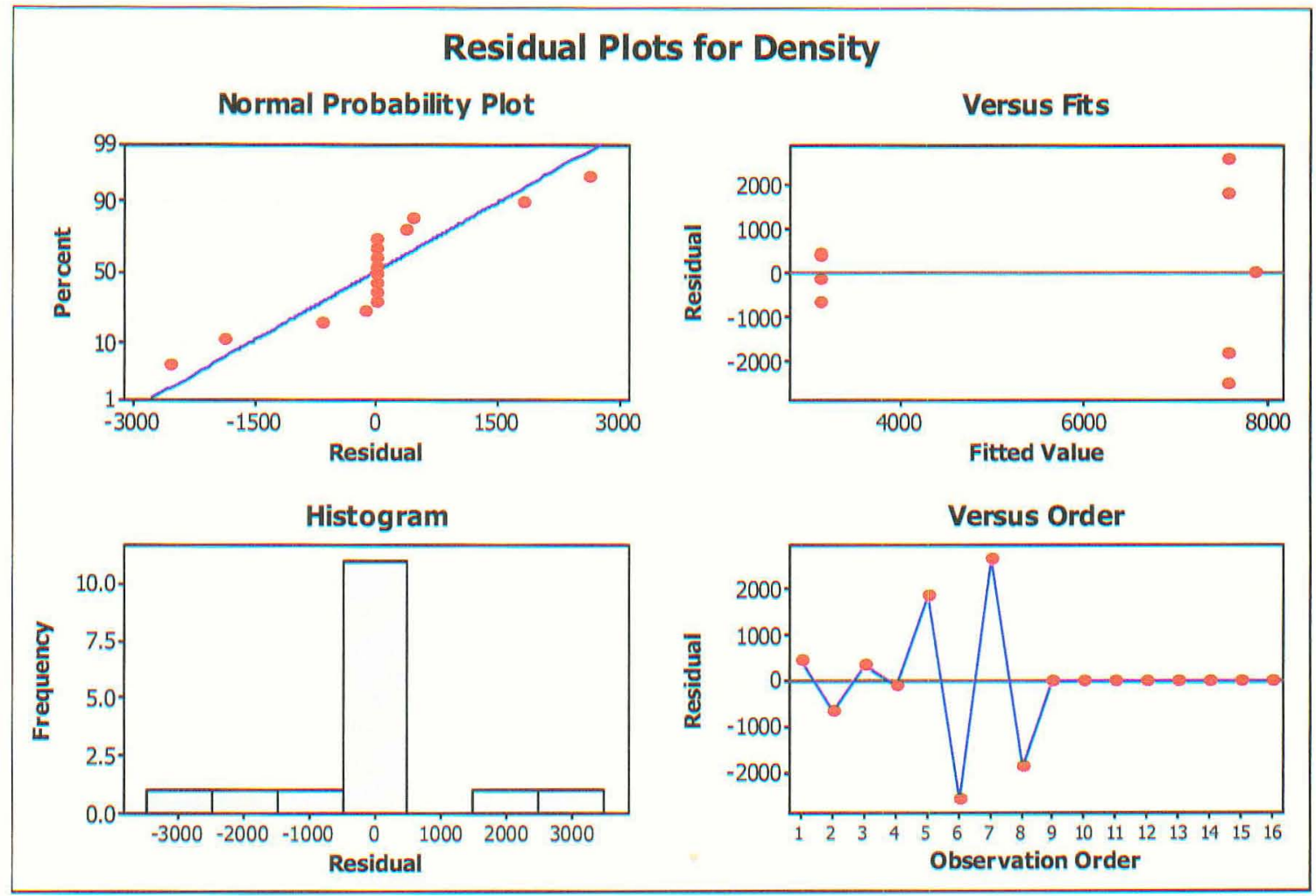



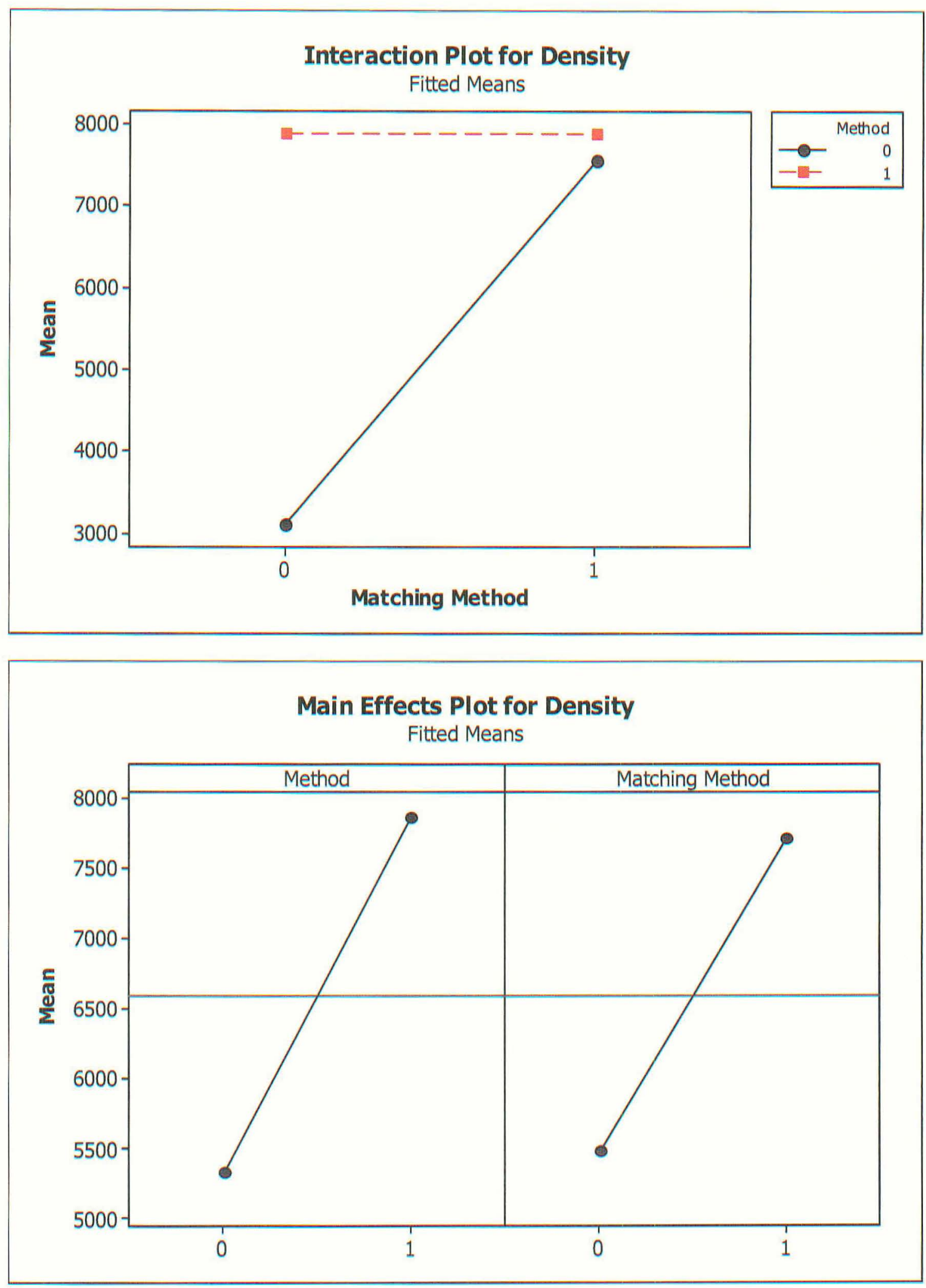


\title{
CURRICULUM VITAE
}

\author{
NAME: $\quad$ Basheer Hawwash
}

$\begin{array}{ll}\text { ADDRESS: } & 3012 \text { Hikes Lane } \\ & \text { Louisville, KY, 40220 } \\ \text { DOB } & \text { April 30, 1984 } \\ \text { EDUCATION } & \text { B.S. Computer Information Systems } \\ & \text { Jordan University of Science and Technology } \\ & \text { Irbid, Jordan } \\ & \text { 2002-2006 }\end{array}$

M.S. Computer Science

University of Louisville

Louisville, KY, USA

2006-2008 